\title{
Concepts in High Temperature Superconductivity
}

\author{
E. W. Carlson, V. J. Emery, S. A. Kivelson, and D. Orgad
}

\section{Preface}

It is the purpose of this paper to explore the theory of high temperature superconductivity. Much of the motivation for this comes from the study of cuprate high temperature superconductors. However, we do not focus in great detail on the remarkable and exciting physics that has been discovered in these materials. Rather, we focus on the core theoretical issues associated with the mechanism of high temperature superconductivity. Although our discussions of theoretical issues in a strongly correlated superconductor are intended to be self contained and pedagogically complete, our discussions of experiments in the cuprates are, unfortunately, considerably more truncated and impressionistic.

Our primary focus is on physics at intermediate temperature scales of order $T_{c}$ (as well as the somewhat larger "pseudogap" temperature) and energies of order the gap maximum, $\Delta_{0}$. Consequently (and reluctantly) we have omitted any detailed discussion of a number of fascinating topics in cuprate superconductivity, including the low energy physics associated with nodal quasiparticles, the properties of the vortex matter which results from the application of a magnetic field, the effects of disorder, and a host of material specific issues. This paper is long enough as it is! 


\section{Contents}

$\begin{array}{ll}\text { 1. Introduction } & \mathbf{7}\end{array}$

We highlight our main themes: mesoscale structure and the need for a kinetic energy driven mechanism.

\section{High Temperature Superconductivity is Hard to Attain}

We explore the reasons why high temperature superconductivity is so difficult to achieve from the perspective of the BCS-Eliashberg approach. Because of retardation, increasing the frequency of the intermediate boson cannot significantly raise $T_{c}$. Strong coupling tends to reduce the phase ordering temperature while promoting competing instabilities.

2.1 Effects of the Coulomb repulsion and retardation on pairing $\quad 10$

$\begin{array}{ll}2.2 \text { Pairing vs. phase ordering } & 12\end{array}$

$\begin{array}{ll}2.3 \text { Competing orders } & 13\end{array}$

\section{Superconductivity in the Cuprates: General Considerations 15}

Some of the most important experimental facts concerning the cuprate high temperature superconductors are described with particular emphasis on those which indicate the need for a new approach to the mechanism of high temperature superconductivity. A perspective on the pseudogap phenomena and the origin of d-wave-like pairing is presented.

$\begin{array}{ll}3.1 \text { A Fermi surface instability requires a Fermi surface } & 17\end{array}$

$\begin{array}{ll}3.2 \text { There is no room for retardation } & 17\end{array}$

$\begin{array}{ll}3.3 \text { Pairing is collective! } & 18\end{array}$

3.4 What determines the symmetry of the pair wavefunction? 19

3.5 What does the pseudogap mean? 21

3.5.1 What experiments define the pseudogap? 21

3.5.2 What does the pseudogap imply for theory? 27

4. Preview: Our View of the Phase Diagram 30

We briefly sketch our view of how the interplay between stripe and superconducting order leads to high temperature superconductivity, various pseudogap phenomena, and non-Fermi liquid behaviors that resemble the physics of the $1 D$ electron gas. This serves as a trailer for Section 13.

\section{Quasi-1D Superconductors}

The well developed theory of quasi-one dimensional superconductors is introduced as the best theoretical laboratory for the study of strongly correlated 
electron fluids. The normal state is a non-Fermi liquid, in which the electron is fractionalized. It can exhibit a broad pseudogap regime for temperatures above $T_{c}$ but below the high temperature Tomonaga-Luttinger liquid regime. $T_{c}$ marks a point of dimensional crossover, where familiar electron quasiparticles appear with the onset of long range superconducting phase coherence.

5.1 Elementary excitations of the 1DEG 33

5.2 Spectral functions of the 1DEG - signatures of fractionalization 39

5.3 Dimensional crossover in a quasi-1D superconductor $\quad 45$

5.3.1 Interchain coupling and the onset of order 45

5.3.2 Emergence of the quasiparticle in the ordered state $\quad 47$

$\begin{array}{ll}5.4 \text { Alternative routes to dimensional crossover } & 50\end{array}$

6. Quasi-1D Physics in a Dynamical Stripe Array 50

An interesting generalization of the quasi-1D system occurs when the background geometry on which the constituent 1DEG's reside is itself dynamically fluctuating. This situation arises in conducting stripe phases.

6.1 Ordering in the presence of quasi-static stripe fluctuations $\quad 51$

6.2 The general smectic fixed point 53

7. Electron Fractionalization in $D>1$ as a Mechanism of High Temperature Superconductivity $\quad 55$

Spin-charge separation offers an attractive route to high temperature superconductivity. It occurs robustly in $1 D$, but is now known to occur in higher dimensions as well, although seemingly only under very special circumstances.

7.1 RVB and spin-charge separation in two dimensions 56

7.2 Is an insulating spin liquid ground state possible in $D>1 ? \quad 57$

$\begin{array}{ll}7.3 \text { Topological order and electron fractionalization } & 59\end{array}$

8. Superconductors with Small Superfluid Density 59

In contrast to conventional superconductors, in superconductors with small superfluid density, fluctuations of the phase of the superconducting order parameter affect the properties of the system in profound ways.

8.1 What ground state properties predict $T_{c}$ ? 59

8.2 An illustrative example: granular superconductors 62

8.3 Classical phase fluctuations $\quad 66$

8.3.1 Superconductors and classical XY models 66

8.3.2 Properties of classical XY models $\quad 67$

$\begin{array}{ll}8.4 \text { Quantum considerations } & 70\end{array}$ 
$\begin{array}{ll}\text { 8.5 Applicability to the cuprates } & 71\end{array}$

$\begin{array}{ll}\text { 8.5.1 } T_{c} \text { is unrelated to the gap in underdoped cuprates } & 72\end{array}$

8.5.2 $T_{c}$ is set by the superfluid density in underdoped cuprates 72

8.5.3 Experimental signatures of phase fluctuations 72

\section{Lessons from Weak Coupling}

The weak coupling renormalization group approach to the Fermi liquid and the $1 D E G$ is presented. The role of retardation, the physics of the Coulomb pseudopotential, and the nonrenormalization of the electron-phonon coupling in a BCS superconductor are systematically derived. The strong renormalization of the electron-phonon interaction in the $1 D E G$ is contrasted with this - it is suggested that this may be a more general feature of non-Fermi liquids.

9.1 Perturbative RG approach in $D>1$

$\begin{array}{ll}9.2 \text { Perturbative RG approach in } D=1 & 77\end{array}$

9.2.1 The one loop beta function $\quad 77$

$\begin{array}{ll}\text { 9.2.2 Away from half filling } & 78\end{array}$

$\begin{array}{ll}9.2 .3 \text { Half filling } & 80\end{array}$

10. Lessons from Strong Coupling $\quad 80$

In certain special cases, well controlled analytic results can be obtained in the limit in which the bare electron-electron and/or electron-phonon interactions are strong. We discuss several such cases, and in particular we demonstrate a theoretically well established mechanism in one dimension, the "spin gap proximity effect," by which strong repulsive interactions between electrons can result in a large and robust spin gap and strongly enhanced local superconducting correlations. We propose this as the paradigmatic mechanism of high temperature superconductivity.

10.1 The Holstein model of interacting electrons and phonons $\quad 80$ 10.1.1 Adiabatic limit: $E_{F} \gg \omega_{D} \quad 81$

10.1.2 Inverse adiabatic limit; negative $U$ Hubbard model $\quad 81$

10.1.3 Large $U_{\text {eff }}$ : bipolarons $\quad 82$

10.2 Insulating quantum antiferromagnets $\quad 83$

10.2.1 Quantum antiferromagnets in more than one dimension 83

10.2.2 Spin gap in even leg Heisenberg ladders 85

$\begin{array}{ll}10.3 \text { The isolated square } & 87\end{array}$

$\begin{array}{ll}\text { 10.4 The spin gap proximity effect mechanism } & 90\end{array}$

11. Lessons from Numerical Studies of Hubbard and Related Models 
The careful use of numerical studies to understand the physics on scales relevant to the mechanism of high temperature superconductivity is advocated.

11.1 Properties of doped ladders $\quad 94$

11.1.1 Spin gap and pairing correlations 94

11.1.2 Phase separation and stripe formation in ladders $\quad 103$

11.2 Properties of the two dimensional $t-J$ model 106

11.2.1 Phase separation and stripe formation 106

11.2.2 Superconductivity and stripes 111

\section{Doped Antiferromagnets}

113

There are many indications that the cuprate superconductors should be viewed as doped antiferromagnetic insulators. The motion of dilute holes in an aniterromagnet is highly frustrated, and attempts to understand the implications of this problem correspondingly frustrating. However, one generic solution is macroscopic or microscopic phase separation into hole poor antiferromagnetic regions and hole rich metallic regions.

12.1 Frustration of the motion of dilute holes in an antiferromagnet 114

12.1.1 One hole in an antiferromagnet $\quad 116$

12.1.2 Two holes in an antiferromagnet $\quad 116$

12.1.1 Many holes: phase separation $\quad 118$

12.2 Coulomb frustrated phase separation and stripes $\quad 121$

12.3 Avoided critical phenomena $\quad 123$

12.4 The cuprates as doped antiferromagnets $\quad 125$

12.4.1 General considerations $\quad 125$

$\begin{array}{ll}12.4 .2 \text { Stripes } & 126\end{array}$

12.5 Additional considerations and alternative perspectives $\quad 127$

$\begin{array}{ll}\text { 12.5.1 Phonons } & 127\end{array}$

12.5.2 Spin-Peierls order $\quad 127$

12.5.3 Stripes in other systems 128

\section{Stripes and High Temperature Superconductivity 128}

We present a coherent view - our view - of high temperature superconductivity in the cuprate superconductors. This section is more broadly phenomenological than is the rest of this paper.

13.1 Experimental signatures of stripes 130

13.1.1 Where do stripes occur in the phase diagram? $\quad 130$

13.2 Stripe crystals, fluids, and electronic liquid crystals 134 
13.3 Our view of the phase diagram-Reprise 137

$\begin{array}{ll}\text { 13.3.1 Pseudogap scales } & 138\end{array}$

13.3.1 Dimensional crossovers 138

13.3.2 The cuprates as quasi-1D superconductors $\quad 139$

13.3.3 Inherent competition $\quad 140$

13.4 Some open questions $\quad 141$

13.4.1 Are stripes universal in the cuprate superconductors? 141

13.4.2 Are stripes an unimportant low temperature complication?

142

13.4.3 Are the length and time scales reasonable? 143

13.4.4 Are stripes conducting or insulating? 143

13.4.5 Are stripes good or bad for superconductivity? 144

13.4.6 Do stripes produce pairing? 145

13.4.7 Do stripes really make the electronic structure quasi-1D? 146

13.4.8 What about overdoping? 147

13.4.9 How large is the regime of substantial fluctuation superconductivity?

13.4.10 What about phonons? 149

13.4.11 What are the effects of quenched disorder? 149

List of Symbols $\quad 151$ 


\section{Introduction}

Conventional superconductors are good metals in their normal states, and are well described by Fermi liquid theory. They also exhibit a hierarchy of energy scales, $E_{F} \gg \hbar \omega_{D} \gg k_{B} T_{c}$, where $E_{F}$ and $\hbar \omega_{D}$ are the Fermi and Debye energies, respectively, and $T_{c}$ is the superconducting transition temperature. Moreover, one typically does not have to think about the interplay between superconductivity and any other sort of collective ordering, since in most cases the only weak coupling instability of a Fermi liquid is to superconductivity. These reasons underlie the success of the BCS-Eliashberg-Migdal theory in describing metallic superconductors [1].

By contrast, the cuprate high temperature superconductors [2] (and various other newly discovered materials with high superconducting transition temperatures) are highly correlated "bad metals," [3, 4] with normal state properties that are not at all those of a Fermi liquid. There is compelling evidence that they are better thought of as doped Mott insulators, rather than as strongly interacting versions of conventional metals [5]. The cuprates also exhibit numerous types of low temperature order which interact strongly with the superconductivity, the most prominent being antiferromagnetism and the unidirectional charge and spin density wave "stripe" order. These orders can compete or coexist with superconductivity. Furthermore, whereas phase fluctuations of the superconducting order parameter are negligibly small in conventional superconductors, fluctuation effects are of order one in the high temperature superconductors because of their much smaller superfluid stiffness.

Apparently, none of this complicates the fundamental character of the superconducting order parameter: it is still a charge $2 \mathrm{e}$ scalar field, although it transforms according to a nontrivial representation of the point group symmetry of the crystal - it is a " $d$-wave superconductor." At asymptotically low temperatures and energies, there is every reason to expect that the physics is dominated by nodal quasiparticles that are similar to those that one might find in a BCS superconductor of the same symmetry. Indeed, there is considerable direct experimental evidence that this expectation is realized [8 11]. However, the failure of Fermi liquid theory to describe the normal state and the presence of competing orders necessitates an entirely different approach to understanding much of the physics, especially at intermediate scales of order $k_{B} T_{c}$, which is the relevant scale for the mechanism of high temperature superconductivity.

It is the purpose of this paper to address the physics of high temperature superconductivity at these intermediate scales. We pay particular attention to the problem of charge dynamics in doped Mott insulators. We also stress the physics of quasi-one dimensional superconductors, in part because that is the one theoretically well understood limit in which superconductivity emerges from a non-Fermi liquid normal state. To the extent that the physics evolves adiabatically from the quasi-one to the quasi-two dimensional

The virtues of $B C S$ theory are extolled.

The assumptions of $B C S$ theory are violated by the high temperature superconductors.

The purpose of this paper. 
limit, this case provides considerable insight into the actual problem of interest. The soundness of this approach can be argued from the observation that $\mathrm{YBa}_{2} \mathrm{Cu}_{3} \mathrm{O}_{7-\delta}(\mathrm{YBCO}$ ) (which is strongly orthorhombic) exhibits very similar physics to that of the more tetragonal cuprates. Since the conductivity and the superfluid density in YBCO exhibit a factor of 2 or greater anisotropy within the plane, 12, 13] this material is already part way toward the quasi-one dimensional limit without substantial changes in the physics! In the second place, because of the delicate interplay between stripe and superconducting orders observed in the cuprates, it is reasonable to speculate that the electronic structure may be literally quasi-one dimensional at the local level, even when little of this anisotropy is apparent at the macroscopic scale.

Mesoscale electronic structure is emphasized.

A kinetic energy driven mechanism is called for.

A prominent theme of this article is the role of mesoscale structure 14. Because the kinetic energy is strongly dominant in good metals, their wavefunctions are very rigid and hence the electron density is highly homogeneous in real space, even in the presence of a spatially varying external potential (e.g. disorder). In a highly correlated system, the electronic structure is much more prone to inhomogeneity [15 17], and intermediate scale structures (stripes are an example) are likely an integral piece of the physics. Indeed, based on the systematics of local superconducting correlations in exact solutions of various limiting models and in numerical "experiments" on $t-J$ and Hubbard models, we have come to the conclusion that mesoscale structure may be essential to a mechanism of high temperature superconducting pairing. (See Sections 10 and 11.) This is a potentially important guiding principle in the search for new high temperature superconductors.

This is related to a concept that we believe is central to the mechanism of high temperature superconductivity: the condensation is driven by a lowering of kinetic energy. A Fermi liquid normal state is essentially the ground state of the electron kinetic energy, so any superconducting state which emerges from it must have higher kinetic energy. The energy gain which powers the superconducting transition from a Fermi liquid must therefore be energy of interaction - this underlies any BCS-like approach to the problem. In the opposite limit of strong repulsive interactions between electrons, the normal state has high kinetic energy. It is thus possible to conceive of a kinetic energy driven mechanism of superconductivity, in which the strong frustration of the kinetic energy is partially relieved upon entering the superconducting state 18 24]. Such a mechanism does not require subtle induced attractions, but derives directly from the strong repulsion between electrons. As will be discussed in Section 10, the proximity effect in the conventional theory of superconductivity is a prototypical example of such a kinetic energy driven mechanism: when a superconductor and a normal metal are placed in contact with each other, the electrons in the metal pair (even if the interactions between them are repulsive) in order to lower their zero point kinetic energy by delocalizing across the interface. A related phenomenon, which we have called 
the "spin gap proximity effect" 20,25] (see Section 10.4), produces strong superconducting correlations in $t-J$ and Hubbard ladders [26], where the reduction of kinetic energy transverse to the ladder direction drives pairing. It is unclear to us whether experiments can unambiguously distinguish between a potential energy and a kinetic energy driven mechanism. TBut since the interaction between electrons is strongly repulsive for the systems in question, we feel that the a priori case for a kinetic energy driven mechanism is very strong.

Our approach in this article is first to analyze various aspects of high temperature superconductivity as abstract problems in theoretical physics, and then to discuss their specific application to the cuprate high temperature superconductors. [ We have also attempted to make each section self contained. Although many readers no doubt will be drawn to read this compelling article in its entirety, we have also tried to make it useful for those readers who are interested in learning about one or another more specific issue. The first eleven sections focus on theoretical issues, except for Section 4 , where we briefly sketch the mechanism in light of our view of the phase diagram of the cuprate superconductors. In the final section, we focus more directly on the physics of high temperature superconductivity in the cuprates, and summarize some of the experimental issues that remain, in our opinion, unsettled. Except where dimensional arguments are important, we will henceforth work with units in which $\hbar=k_{B}=1$.

\section{High Temperature Superconductivity is Hard to Attain}

Superconductivity in metals is the result of two distinct quantum phenomena:

Catch 22 pairing and long range phase coherence. In conventional homogeneous superconductors, the phase stiffness is so great that these two phenomena occur simultaneously. On the other hand, in granular superconductors and Josephson junction arrays, pairing occurs at the bulk transition temperature of the constituent metal, while long range phase coherence, if it occurs at all, obtains at a much lower temperature characteristic of the Josephson coupling between superconducting grains. To achieve high temperature superconductivity requires that both scales be elevated simultaneously. However, given that the bare interactions between electrons are strongly repulsive, it is somewhat miraculous that electron pairing occurs at all. Strong interactions, which might enable pairing at high scales, typically also have the effect of strongly

\footnotetext{
${ }^{1}$ Recent papers by Molegraaf et al 27] and Santadner-Syro et al [28] present very plausible experimental evidence of a kintetic energy driven mechanism of superconductivity in at least certain high temperature superconductors.

${ }^{2}$ While examples of similar behavior can be found in other materials, for ease of exposition we have focused on this single example.
}

The plan of the article is discussed.

$\hbar=1$

$k_{B}=1$. 
$B C S$ is not for high $T_{c} \quad$ superconductivity.

Never forget the Coulomb interaction. suppressing the phase stiffness, and moreover typically induce other orders in the system which compete with superconductivity.

It is important in any discussion of the theory of high temperature superconductivity to have clearly in mind why conventional metallic superconductors, which are so completely understood in the context of the Fermi liquid based BCS-Eliashberg theory, rarely have $T_{c}$ 's above $15 \mathrm{~K}$, and never above $30 \mathrm{~K}$. In this section, we briefly discuss the principal reasons why a straightforward extension of the BCS-Eliashberg theory does not provide a framework for understanding high temperature superconductivity, whether in the cuprate superconductors, or in $\mathrm{C}_{60}$, or possibly even $\mathrm{BaKBiO}$ or $\mathrm{MgB}_{2}$.

\subsection{Effects of the Coulomb repulsion and retardation on pairing}

In conventional BCS superconductors, the instantaneous interactions between electrons are typically repulsive (or at best very weakly attractive) - it is only because the phonon induced attraction is retarded that it (barely) dominates at low frequencies. Even if new types of intermediate bosons are invoked to replace phonons in a straightforward variant of the BCS mechanism, the instantaneous interactions will still be repulsive, so any induced attraction is typically weak, and only operative at low frequencies.

Strangely enough, the deleterious effects of the Coulomb interaction on high temperature superconductivity has been largely ignored in the theoretical literature. The suggestion has been made that high pairing scales can be achieved by replacing the relatively low frequency phonons which mediate the pairing in conventional metals by higher frequency bosonic modes, such as the spin waves in the high temperature superconductors [29 32] or the shape modes [33, 34] of $\mathrm{C}_{60}$ molecules. However, in most theoretical treatments of this idea, the Coulomb pseudopotential is either neglected or treated in a cavalier manner. That is, models are considered in which the instantaneous interactions between electrons are strongly attractive. This is almost certainly [14, 20, 35 37] an unphysical assumption!

In Section 9, we use modern renormalization group ( $\mathrm{RG}$ ) methods [38, 39] to derive the conventional expression for the Coulomb pseudopotential, and how it enters the effective pairing interaction at frequencies lower than the Debye frequency, $\omega_{D}$. This theory is well controlled so long as $\omega_{D} \ll E_{F}$ and the interaction strengths are not too large. It is worth reflecting on a well known, but remarkably profound result that emerges from this analysis: As electronic states are integrated out between the microscopic scale $E_{F}$ and the intermediate scale, $\omega_{D}$, the electron-phonon interaction is unrenormalized (and so can be well estimated from microscopic considerations), but the Coulomb repulsion is reduced from a bare value, $\mu$, to a renormalized value,

$$
\mu^{*}=\mu /\left[1+\mu \log \left(E_{F} / \omega_{D}\right)\right] .
$$

\footnotetext{
${ }^{3}$ I.e. magnetic, structural, etc.
} 
Here, as is traditional, $\mu$ and $\mu^{*}$ are the dimensionless measures of the interaction strength obtained by multiplying the interaction strength by the density of states. We define $\lambda$ in an analogous manner for the electronphonon interaction. Thus, even if the instantaneous interaction is repulsive (i.e. $\lambda-\mu<0$ ), the effective interaction at the scale $\omega_{D}$ will nonetheless be attractive $\left(\lambda-\mu^{*}>0\right)$ for $\omega_{D} \ll E_{F}$. Below this scale, the standard RG analysis yields the familiar weak coupling estimate of the pairing scale $T_{p}$ :

$$
T_{p} \sim \omega_{D} \exp \left[-1 /\left(\lambda-\mu^{*}\right)\right]
$$

The essential role of retardation is made clear if one considers the dependence of $T_{p}$ on $\omega_{D}$ :

$$
\frac{d \log \left[T_{p}\right]}{d \log \left[\omega_{D}\right]}=1-\left[\mu^{*} \log \left(\frac{T_{p}}{\omega_{D}}\right)\right]^{2} .
$$

So long as $\omega_{D} \ll E_{F} \exp [-(1-\lambda) / \lambda \mu]$, we have $\frac{d \log \left[T_{p}\right]}{d \log \left[\omega_{D}\right]} \approx 1$, and $T_{p}$ is a linearly rising function of $\omega_{D}$, giving rise to the conventional isotope effect.1 However, when $\omega_{D}>T_{p} \exp \left[1 / \mu^{*}\right]$, we have $\frac{d \log \left[T_{p}\right]}{d \log \left[\omega_{D}\right]}<0$, and $T_{p}$ becomes a decreasing function of $\omega_{D}$ ! Clearly, unless $\omega_{D}$ is exponentially smaller than $E_{F}$, superconducting pairing is impossible by the conventional mechanism 5 .

This problem is particularly vexing in the cuprate high temperature superconductors and similar materials, which have low electron densities, and incipient or apparent Mott insulating behavior. This means that screening of the Coulomb interaction is typically poor, and $\mu$ is thus expected to be large. Specifically, from the inverse Fourier transform of the $\boldsymbol{k}$ dependent gap function measured [40] in angle resolved photoemission spectroscopy (ARPES) on $\mathrm{Bi}_{2} \mathrm{Sr}_{2} \mathrm{CaCu}_{2} \mathrm{O}_{8+\delta}$, it is possible to conclude (at least at the level of the $\mathrm{BCS}$ gap equation) that the dominant pairing interactions have a range equal to the nearest neighbor copper distance. Since this distance is less than the distance between doped holes, it is difficult to believe that metallic screening is very effective at these distances. From cluster calculations and an analysis of various local spectroscopies, a crude estimate [20] of the Coulomb repulsion at this distance is of order $0.5 \mathrm{eV}$ or more. To obtain pairing from a conventional mechanism with relatively little retardation, it is necessary that the effective attraction be considerably larger than this!

We are therefore led to the conclusion that the only way a BCS mechanism can produce a high pairing scale is if the effective attraction, $\lambda$, is very large indeed. This, however, brings other problems with it.

\footnotetext{
${ }^{4}$ Recall, for phonons, $d \log \left[\omega_{D}\right] / d \log [M]=-1 / 2$.

${ }^{5}$ In the present discussion we have imagined varying $\omega_{D}$ while keeping fixed the electron-phonon coupling constant, $\lambda=\frac{C}{M \omega_{D}^{2}}=\frac{C}{K}$, where $C$ is proportional to the (squared) gradient of the electron-ion potential and $K$ is the "spring constant" between the ions. If we consider instead the effect of increasing $\omega_{D}$ at fixed $C / M$, it leads to a decrease in $\lambda$ and hence a very rapid suppression of the pairing scale.
}

Retardation is an essential feature of the BCS mechanism.

Pairing's Bane 


\section{2 $\quad$ Pairing vs. phase ordering}

In most cases, it is unphysical to assume the existence of strong attractive interactions between electrons. However, even supposing we ignore this, strong attractive interactions bring about other problems for high temperature superconductivity: 1) There is a concomitant strong reduction of the phase ordering temperature and thus of $T_{c}$. 2) There is the possibility of competing orders. We discuss the first problem here, and the second in Section 2.3.

Strong attractive interactions typically result in a large increase in the effective mass, and a corresponding reduction of the phase ordering temperature. Consider, for example, the strong coupling limit of the negative $U$ Hubbard model 411] or the Holstein model 442], discussed in Section 10. In both cases, pairs have a large binding energy, but they typically Bose condense at a very low temperature because of the large effective mass of a tightly bound pair - the effective mass is proportional to $|U|$ in the Hubbard model and is exponentially large in the Holstein model. (See Section 10.)

Whereas in conventional superconductors, the bare superfluid stiffness is

Phase ordering is a serious business in the cuprates.

A general principle is proposed: "optimal" $T_{c}$ occurs as a crossover. so great that even a substantial renormalization of the effective mass would hardly matter, in the cuprate high temperature superconductors, the superfluid stiffness is small, and a substantial mass renormalization would be catastrophic. The point can be made most simply by considering the result of simple dimensional analysis. The density of doped holes per plane in an optimally doped high temperature superconductor is approximately $n_{2 d}=10^{14} \mathrm{~cm}^{-2}$. Assuming a density of hole pairs that is half this, and taking the rough estimate for the pair effective mass, $m^{*}=2 m_{e}$, we find a phase ordering scale,

$$
T_{\theta}=\hbar^{2} n_{2 d} / 2 m^{*} \approx 10^{-2} e V \approx 100 K .
$$

Since this is in the neighborhood of the actual $T_{c}$, it clearly implies that any large mass renormalization would be incompatible with a high transition temperature. What about conventional superconductors? A similar estimate in a $W=10 \AA$ thick $\mathrm{Pb}$ film gives $T_{\theta}=\hbar^{2} n_{3 d} W / 2 m^{*} \approx 1 \mathrm{eV} \approx 10,000 \mathrm{~K}$ ! Clearly, phase fluctuations are unimportant in $\mathrm{Pb}$. This issue is addressed in detail in Section 8.

We have seen how $T_{p}$ and $T_{\theta}$ have opposite dependence on coupling strength. If this is a general trend, then it is likely that any material in which $T_{c}$ has been optimized has effectively been tuned to a crossover point between pairing and condensation. A modification of the material which produces stronger effective interactions will increase phase fluctuations and thereby reduce $T_{c}$, while weaker interactions will lower $T_{c}$ because of pair breaking. In Section 8 it will be shown that optimal doping in the cuprate superconductors corresponds to precisely this sort of crossover from a regime in which $T_{c}$ is determined by phase ordering to a pairing dominated regime. 


\subsection{Competing orders}

A Fermi liquid is a remarkably robust state of matter. In the absence of nesting, it is stable for a range of repulsive interactions; the Cooper instability is its only weak coupling instability. The phase diagram of simple metals consists of a high temperature metallic phase and a low temperature superconducting state. When the superconductivity is suppressed by either a magnetic field or appropriate disorder (e.g. paramagnetic impurities), the system remains metallic down to the lowest temperatures.

The situation becomes considerably more complex for sufficiently strong interactions between electrons. In this case, the Fermi liquid description of the normal or high temperature phase breaks down and many possible phases compete. In addition to metallic and superconducting phases, one would generally expect various sorts of electronic "crystalline" phases, including charge ordered phases (i.e. a charge density wave - CDW - of which the Wigner crystal is the simplest example) and spin ordered phases (i.e. a spin density wave - SDW - of which the Néel state is the simplest example).

Typically, one thinks of such phases as insulating, but it is certainly possible for charge and spin order to coexist with metallic or even superconducting electron transport. For example, this can occur in a conventional weak coupling theory if the density wave order opens a gap on only part of the Fermi surface, leaving other parts gapless [43]. It can also occur in a multicomponent system, in which the density wave order involves one set of electronic orbitals, and the conduction occurs through others - this is the traditional understanding of the coexisting superconducting and magnetic order in the Chevrel compounds [44].

Such coexistence is also possible for less conventional orders. One particular class of competing orders is known loosely as "stripe" order. Stripe order refers to unidirectional density wave order, i.e. order which spontaneously breaks translational symmetry in one direction but not in others. We will refer to charge stripe order, if the broken symmetry leads to charge density modulations and spin stripe order if the broken symmetry leads to spin density modulations, as well. Charge stripe order can occur without spin order, but spin order (in a sense that will be made precise, below) implies charge order [45]. Both are known on theoretical and experimental grounds to be a prominent feature of doped Mott insulators in general, and the high temperature superconductors in particular [6, 46 51]. Each of these orders can occur in an insulating, metallic, or superconducting state.

In recent years there has been considerable theoretical interest in other types of order that could be induced by strong interactions. From the perspective of stripe phases, it is natural to consider various partially melted "stripe liquid" phases, and to classify such phases, in analogy with the classification of phases of classical liquid crystals, according to their broken sym-

\footnotetext{
${ }^{6}$ As long as the interactions are not too strong.

${ }^{7}$ Whether it breaks down for fundamental or practical reasons is unimportant.
} 
Competition matters...

... and so does symbiosis. metries [52]. For instance, one can imagine a phase that breaks rotational symmetry (or, in a crystal, the point group symmetry) but not translational symmetry, i.e. quantum (ground state) analogues of nematic or hexatic liquid crystalline phases. Still more exotic phases, such as those with ground state orbital currents [53 58] or topological order 59, have also been suggested as the explanation for various observed features of the phenomenology of the high temperature superconductors.

Given the complex character of the phase diagram of highly correlated electrons, it is clear that the conventional approach to superconductivity, which focuses solely on the properties of the normal metal and the pure superconducting phase, is suspect. A more global approach, which takes into account some (or all) of the competing phases is called for. Moreover, even the term "competing" carries with it a prejudice that must not be accepted without thought. In a weakly correlated system, in which any low temperature ordered state occurs as a Fermi surface instability, different orders generally do compete: if one order produces a gap on part of the Fermi surface, there are fewer remaining low energy degrees of freedom to participate in the formation of another type of order. For highly correlated electrons, however, the sign of the interaction between different types of order is less clear. It can happen [60] that under one set of circumstances, a given order tends to enhance superconductivity and under others, to suppress it.

The issue of competing orders, of course, is not new. In a Fermi liquid, strong effective attractions typically lead to lattice instabilities, charge or spin density wave order, etc. Here the problem is that the system either becomes an insulator or, if it remains metallic, the residual attraction is typically weak. For instance, lattice instability has been seen to limit the superconducting transition temperature of the A15 compounds, the high temperature superconductors of a previous generation. Indeed, the previous generation of BCS based theories which addressed the issue always concluded that competing orders suppress superconductivity 44.

More recently it has been argued that near an instability to an ordered state there is a low lying collective mode (the incipient Goldstone mode of the ordered phase) which can play the role of the phonon in a BCS-like mechanism of superconductivity [29, 61, 62. In an interesting variant of this idea, it has been argued that in the neighborhood of a zero temperature transition to an ordered phase, quantum critical fluctuations can mediate superconducting pairing in a more or less traditional way 63 65. There are reasons to expect this type of fluctuation mediated pair binding to lead to a depression of $T_{c}$. If the collective modes are nearly Goldstone modes (as opposed to relaxational "critical modes"), general considerations governing the couplings of such modes in the ordered phase imply that the superconducting transition temperature is depressed substantially from any naive estimate by large vertex corrections [66]. Moreover, in a regime of large fluctuations to a nearby ordered phase, one generally expects a density of states reduction 
due to the development of a pseudogap; feeding this psuedogapped density of states back into the BCS-Eliashberg theory will again result in a significant reduction of $T_{c}$.

\section{Superconductivity in the Cuprates: General Considerations}

While the principal focus of the present article is theoretical, the choice of topics and models and the approaches are very much motivated by our interest in the experimentally observed properties of the cuprate high temperature superconductors. In this section, we discuss briefly some of the most dramatic (and least controversial) aspects of the phenomenology of these materials, and what sorts of constraints those observations imply for theory. As we are primarily interested in the origin of high temperature superconductivity, we will deal here almost exclusively with experiments in the temperature and energy ranges between about $T_{c} / 2$ and a few times $T_{c}$.

Before starting, there are a number of descriptive terms that warrant definition. The parent state of each family of the high temperature superconductors is an antiferromagnetic "Mott" insulator with one hole (and spin 1/2) per planar copper. These insulators are transformed into superconductors

\footnotetext{
8 The term "Mott insulator" means many things to many people. One definition is that a Mott insulator is insulating because of interactions between electrons, rather than because a noninteracting band is filled. This is not a precise definition. For example, a Mott insulating state can arise due to a spontaneously broken symmetry which increases the size of the unit cell. However, this is adiabatically connected to the weak coupling limit, and can be qualitatively understood via generalized Hartree-Fock theory. There is still a quantitative distinction between a weak coupling "simple" insulator on the one hand, which has an insulating gap that is directly related to the order parameter which characterizes the broken symmetry, and the "Mott" insulator on the other hand, which has an insulating gap which is large due to the strong repulsion between electrons. In the latter case, the resistivity begins to grow very large compared to the quantum of resistance well above the temperature at which the broken symmetry occurs. The undoped cuprate superconductors are clearly Mott insulators in the quantitative sense that the insulating gap is of order $2 \mathrm{eV}$, while the antiferromagnetic ordering temperatures are around $30 \mathrm{meV}$.

However, for those who prefer [67] a sharp, qualitative distinction, the term "Mott insulator" is reserved for "spin liquid" states which are distinct zero temperature phases of matter, do not break symmetries, and cannot be understood in terms of any straightforward Hartree-Fock description. Many such exotic states have been theoretically envisaged, including the long [5, 68] and short ranged 69 71 RVB liquids, the chiral spin liquid 72 74, the nodal spin liquid 75, 76 and various other fractionalized states with topological order 77,78$]$. Very recently, in the first "proof of principle," a concrete model with a well defined short ranged RVB phase has been discovered [79, 80 .
} 


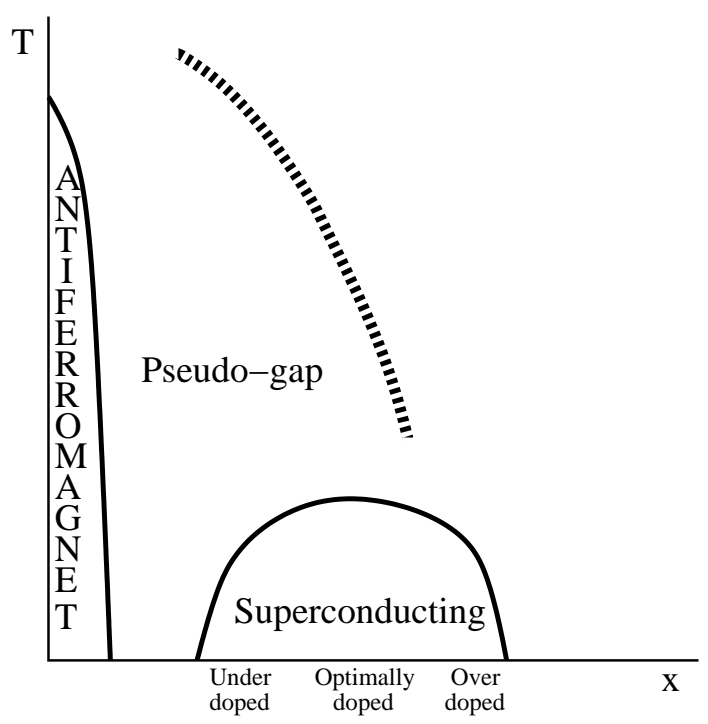

Fig. 1. Schematic phase diagram of a cuprate high temperature superconductor as a function of temperature and $x$ - the density of doped holes per planar $\mathrm{Cu}$. The solid lines represent phase transitions into the antiferromagnetic (AF) and superconducting (SC) states. The dashed line marks the openning of a pseudogap $(\mathrm{PG})$. The latter crossover is not sharply defined and there is still debate on its position; see Refs. 81, 82 .

by introducing a concentration, $x$, of "doped holes" into the copper oxide planes. As a function of increasing $x$, the antiferromagnetic transition temperature is rapidly suppressed to zero, then the superconducting transition temperature rises from zero to a maximum and then drops down again. (See Fig. 1.) Where $T_{c}$ is an increasing function of $x$, the materials are said to be "underdoped." They are "optimally doped" where $T_{c}$ reaches its maximum at $x \approx 0.15$, and they are "overdoped" for larger $x$. In the underdoped regime there are a variety of crossover phenomena observed [81, 32] at temperatures above $T_{c}$ in which various forms of spectral weight at low energies are apparently suppressed - these phenomena are associated with the opening of a "psuedogap." There are various families of high temperature superconductors, all of which have the same nearly square copper oxide planes, but different structures in the regions between the planes. One characteristic that seems to have a fairly direct connection with $T_{c}$ is the number of copper-oxide planes that are close enough to each other that interplane coupling may be significant; $T_{c}$ seems generally to increase with number of planes within a homologous series, at least as one progresses from "single layer" to "bilayer," to "trilayer" materials [. $[83$. 


\subsection{A Fermi surface instability requires a Fermi surface}

As has been stressed, for instance, by Schrieffer [1], BCS theory relies heavily on the accuracy with which the normal state is described by Fermi liquid theory. BCS superconductivity is a Fermi surface instability, which is only a reasonable concept if there is a well defined Fermi surface. BCS-Eliashberg theory relies on the dominance of a certain class of diagrams, summed to all orders in perturbation theory. This can be justified from phase space considerations for a Fermi liquid, but need not be valid more generally. To put it most physically, BCS theory pairs well defined quasiparticles, and therefore requires well defined quasiparticles in the normal state.

There is ample evidence that in optimally and underdoped cuprates, at least, there are no well defined quasiparticles in the normal state. This can be deduced directly from ARPES studies of the single particle spectral function 84 91, or indirectly from an analysis of various spin, current, and density response functions of the system 3, 国. (Many, though not all, of these response functions have been successfully described 92 94 by the "marginal Fermi liquid" phenomenology.) Because we understand the nature of a Fermi liquid so well, it is relatively straightforward to establish that a system is a non-Fermi liquid, at least in extreme cases. It is much harder to establish the cause of this behavior - it could be due to the proximity of a fundamentally new non-Fermi liquid ground state phase of matter, or it could be because the characteristic coherence temperature, below which well defined quasiparticles dominate the physics, is lower than the temperatures of interest. Regardless of the reason for the breakdown of Fermi liquid theory, a description of the physics at scales of temperatures comparable to $T_{c}$ can clearly not be based on a quasiparticle description, and thus cannot rely on BCS theory.

\subsection{There is no room for retardation}

As stressed in Section 2.1, retardation plays a pivotal role in the BCS mechanism. In the typical metallic superconductor, the Fermi energy is of order $10 \mathrm{eV}$, while phonon frequencies are of order $10^{-2} \mathrm{eV}$, so $E_{F} / \omega_{D} \sim 10^{3}$ ! Since the renormalization of the Coulomb pseudopotential is logarithmic, this large value of the retardation is needed. In the cuprate superconductors, the bandwidth measured in ARPES is roughly $E_{F} \approx 0.3 \mathrm{eV}$ - this is a renormalized bandwidth of sorts, but this is presumably what determines the quasiparticle dynamics. Independent of anything else, the induced interaction must clearly be fast compared to the gap scale, $\omega_{D}>2 \Delta_{0}$, where $\Delta_{0}$ is the magnitude of the superconducting gap. From either ARPES 95, 96] or tunnelling [97] experiments, we can estimate $2 \Delta_{0} \approx 0.06 \mathrm{eV}$. Thus, a rough upper bound $E_{F} / \omega_{D}<E_{F} / 2 \Delta_{0} \sim 5$ can be established on how retarded an interaction in the cuprates can possibly be. That is almost not retarded at all!

We belabor the need for a non-Fermi liquid based approach. 


\subsection{Pairing is collective!}

For the most part, the superconducting coherence length, $\xi_{0}$, cannot be directly measured in the high temperature superconductors because, for $T \ll$ $T_{c}$, the upper critical field, $H_{c 2}$, is too high to access readily. However, it can be inferred indirectly 98 102 in various ways, and for the most part people have concluded that $\xi_{0}$ is approximately 2 or 3 lattice constants in typical optimally doped materials. This has lead many people to conclude that these materials are nearly in a "real space pairing" limit [103 107], in which pairs of holes form actual two particle bound states, and then Bose condense at $T_{c}$. This notion is based on the observation that if $x$ is the density of "doped holes" per site, then the number of pairs per coherence area, $N_{p}=(1 / 2) x \pi \xi_{0}^{2} / a^{2}$, is a number which is approximately equal to 1 for "optimal doping," $x \approx 0.15-0.20$.

However, there are strong a priori and empirical reasons to discard this

Real space pairs are dismissed. viewpoint.

On theoretical grounds: In a system dominated by strong repulsive interactions between electrons, it is clear that pairing must be a collective phenomenon. The Coulomb interaction between an isolated pair of doped holes would seem to be prohibitively large, and it seems unlikely that a strong enough effective attraction can emerge to make such a strong binding possible. (Some numerical studies of this have been carried out, in the context of ladder systems, by Dagotto and collaborators [108].) Moreover, it is far from clear that the dimensional argument used above makes any sense: Why should we only count doped holes in making this estimate? What are the rest of the holes doing all this time? If we use the density of holes per site $(1+x)$, which is consistent with the area enclosed by the Fermi surface seen in ARPES [109], the resulting $N_{p}$ is an order of magnitude larger than the above estimate..$^{9}$

On experimental grounds: The essential defining feature of real space pairing is that the chemical potential moves below the bottom of the band. Incipient real space pairing must thus be associated with significant motion of the chemical potential toward the band bottom with pairing 103, 104, 111, 112. However, experimentally, the chemical potential is found to lie in the middle of the band, where the enclosed area of the Brilloin zone satisfies

\footnotetext{
${ }^{9}$ A theory of real space pairs which includes all the electrons and the repulsive interactions between them can be caricatured as a hard core quantum dimer model 70. Here the pairing is collective, due to the high density of pairs. Indeed, $N_{p}$ involves all of the electrons (the doped holes are not paired at all), but the superfluid density is small, involving only the density of doped holes. This contrasts markedly with the case of clean metallic superconductors where the density of pairs (that is, the density of electrons whose state is significantly altered by pairing) is small, $\sim N\left(E_{F}\right) \Delta_{0}$, while the superfluid density is large and involves all the electrons. There is some evidence that the former situation in fact pertains to the high temperature superconductors [110].
} 
Luttinger's theorem, and no significant motion at $T_{c}$ (or at any pseudogap temperature in underdoped materials) has been observed $113 \sqrt{117}$. This fact, alone, establishes that the physics is nowhere near the real space pairing limit.

\subsection{What determines the symmetry of the pair wavefunction?}

Independent of but contemporary with the discovery of high temperature superconductivity in the cuprates, Scalapino, Loh, and Hirsch 118, in a prescient work suggested the possibility of superconductivity in the two dimensional Hubbard model in the neighborhood of the antiferromagnetic state at half filling. This work, which was in spirit a realization of the ideas of Kohn and Luttinger [119], concluded that the dominant superconducting instability should have $d_{\left(x^{2}-y^{2}\right)}$ symmetry, as opposed to $s$ symmetry. Immediately after the discovery of high temperature superconductivity, a large number of other theorists [29, 120 125] came to the same conclusion, based on a variety of purely theoretical analyses, although at the time the experimental evidence of such pairing was ambiguous, at best. By now it seems very clear that this idea was correct, at least for a majority of the cuprate superconductors, based on a variety of phase sensitive measurements [126 128]. This represents one of the great triumphs of theory in this field. (There are still some experiments which appear to contradict this symmetry assignment 129, so the subject cannot be said to be completely closed, but it seems very unlikely that the basic conclusion will be overturned.)

While the names " $s$ " and " $d$ " relate to the rotational symmetries of free space, it is important to understand what is meant by $s$-wave and $d$-wave in a lattice system which, in place of continuous rotational symmetry, has the discrete point group symmetry of the crystal. Consequently, the possible pairing symmetries correspond to the irreducible representations of the point group: singlet orders are even under inversion and triplet orders are odd. In the case of a square crystal 10, the possible singlet orders (all corresponding to one dimensional representations) are colloquially called $s, d_{\left(x^{2}-y^{2}\right)}, d_{(x y)}$, and $g$, and transform like $1,\left(x^{2}-y^{2}\right),(x y)$, and $\left(x^{2}-y^{2}\right)(x y)$, respectively. As a function of angle, the gap parameter in an $s$-wave order always has a unique sign, the $d$-wave gap changes sign four times, and the $g$-wave changes sign 8 times. A fifth type of order is sometimes discussed, called extended-s, in which the gap function changes sign as a function of the magnitude of $\boldsymbol{k}$, rather than as a function of its direction - this is not a true symmetry classification, and in any generic model there is always finite mixing between $s$ and extended $s$.

In crystals with lower symmetry, there are fewer truly distinct irreducible representations. For instance, if the square lattice is replaced with a rect-

$\overline{10}$ The pairing symmetries should really be classified according to the point group of a tetragonal crystal, but since the cuprates are quasi-two dimensional, it is conventional, and probably reasonable, to classify them according to the symmetries of a square lattice.

"d-wave-like" pairing is defined.
Theory has had its triumphs.

$d$-wave pairing is defined. 
angular one, the distinction between $s$ and $d_{\left(x^{2}-y^{2}\right)}$ is lost (they mix), as is that between $d_{(x y)}$ and $g$. On the other hand, if the elementary squares are sheared to form rhombuses, then the $s$ and $d_{(x y)}$ symmetries are mixed, as are $d_{\left(x^{2}-y^{2}\right)}$, and $g$. Both of these lower symmetries correspond to a form of orthorhombic distortion observed in the cuprates - the former is the correct symmetry group for $\mathrm{YBa}_{2} \mathrm{Cu}_{3} \mathrm{O}_{7-\delta}$ and the latter for $\mathrm{La}_{2-x} \mathrm{Sr}_{x} \mathrm{CuO}_{4}$. However, so long as the physics does not change fundamentally as the lattice symmetry is reduced, it is reasonable to classify order parameters as " $d$-wavelike" or " $s$-wave-like." We define an order parameter as being $d$-wave-like if it changes sign under $90^{\circ}$ rotation, although it is only a true $d$-wave if its magnitude is invariant under this transformation. Conversely, it is $s$-wave-like if its sign does not change under this rotation, or when reflected through any approximate symmetry plane. In almost all cases what is really being seen in phase sensitive measurements on the cuprates is that the order parameter is $d$-wave-like. (It is worth noting that in $t-J$ and Hubbard ladders, $d$-wavelike pairing is the dominant form of pairing observed in both analytic and numerical studies, as discussed below.)

Strong repulsion does not necessarily lead to d-wave pairing.
There is a widespread belief that $d$-wave symmetry follows directly from the presence of strong short range interactions between electrons, irrespective of details such as band structure. The essential idea here follows from the observation that the pair wavefunction, at the level of BCS mean field theory, is expressed in terms of the gap parameter, $\Delta_{k}$, and the quasiparticle spectrum, $E_{\boldsymbol{k}}$, as

$$
\phi_{\text {pair }}(\boldsymbol{r})=\sum_{\boldsymbol{k}} \frac{1}{L^{d / 2}} e^{i \boldsymbol{k} \cdot \boldsymbol{r}} \frac{\Delta_{\boldsymbol{k}}}{2 E_{\boldsymbol{k}}} .
$$

In the presence of strong short range repulsion (and weaker longer range attraction) between electrons, it is favorable for $\phi_{\text {pair }}$ to vanish at $\boldsymbol{r}=\mathbf{0}$, which it does automatically if the pairing is not $s$-wave. While this argument makes some physical sense, it is ultimately wrong. In the limit of dilute electrons, where the coherence length is much smaller than the inter-electron distance, the pairing problem reduces to a two particle problem. It is well known that in the continuum the lowest energy two particle spin singlet bound state is nodeless. Given certain mild conditions on the band structure one can also prove it on the lattice 1 . Therefore, in this limit, the order parameter is necessarily $s$-wave-like!

The above discrepancy teaches us that it is the presence of the kinematical constraints imposed by the Fermi sea that allows for non $s$-wave pairing. The ultimate pairing symmetry is a reflection of the distribution in momentum space of the low energy single particle spectral weight. The reason for this is clear within BCS theory where the energy gain, which drives the transition,

11 This is true under conditions that the hopping matrix, i.e. the band structure, satisfies a Peron-Frobenius condition. 
comes from the interaction term

$$
\text { Potential Energy }=\sum_{\boldsymbol{k}, \boldsymbol{k}^{\prime}} V_{\boldsymbol{k}, \boldsymbol{k}^{\prime}} \frac{\Delta_{\boldsymbol{k}}}{2 E_{\boldsymbol{k}}} \frac{\Delta_{\boldsymbol{k}^{\prime}}}{2 E_{\boldsymbol{k}^{\prime}}},
$$

which is maximized by a gap function that peaks in regions of high density of states unless the pairing potential that connects these regions is particularly small. (Although we do not know of an explicit justification of this argument for a non-BCS theory, for example one which is driven by gain in kinetic energy, we feel that the physical consideration behind it is robust.)

Finally, there is another issue which is related to order parameter symmetry in a manner that is more complex than is usually thought - this is the issue of the existence of nodal quasiparticles. While nodal quasiparticles are natural in a $d$-wave superconductor, $d$-wave superconductors can be nodeless, and $s$ wave superconductors can be nodal. To see this, it is possible to work entirely in the weak coupling limit where BCS theory is reliable. The quasiparticle excitation spectrum can thus be expressed as

$$
E_{k}=\sqrt{\varepsilon_{k}^{2}+\Delta_{k}^{2}}
$$

where $\varepsilon_{\boldsymbol{k}}$ is the quasiparticle dispersion in the normal state (measured from the Fermi energy). Nodal quasiparticles occur wherever the Fermi surface, that is the locus of points where $\varepsilon_{\boldsymbol{k}}=0$, crosses a line of gap nodes, the locus of points where $\Delta_{k}=0$. If the Fermi surface is closed around the origin, $\boldsymbol{k}=0$, or about the Brilloin zone center, $\boldsymbol{k}=(\pi, \pi)$ (as it is most likely in optimally doped $\mathrm{Bi}_{2} \mathrm{Sr}_{2} \mathrm{CaCu}_{2} \mathrm{O}_{8+\delta}$ [130]), then the $d$-wave symmetry of $\Delta_{k}=0$ implies the existence of nodes. However, if the Fermi surface were closed about $\boldsymbol{k}=(0, \pi)$ (and symmetry related points), there would be no nodal quasiparticles [131]. Indeed, it is relatively easy to characterize 132, 133 the quantum phase transition between a nodal and nodeless $d$-wave superconductor which occurs as a parameter that alters the underlying band structure is varied. Conversely, it is possible to have lines of gap nodes for an extended $s$-wave superconductor, and if these cross the Fermi surface, the superconductor will posses nodal quasiparticles.

\subsection{What does the pseudogap mean?}

What experiments define the pseudogap? One of the most prominent, and most discussed features of the cuprate superconductors is a set of crossover phenomena [54, 81, 82] which are widely observed in underdoped cuprates and, to various extents, in optimally and even slightly overdoped materials. Among the experimental probes which are used to locate the pseudogap temperature in different materials are:

1) ARPES and c-axis tunnelling: There is a suppression of the low energy single particle spectral weight, shown in Figs. 2 and 3 at temperatures

What's so pseudo about the pseudogap? 


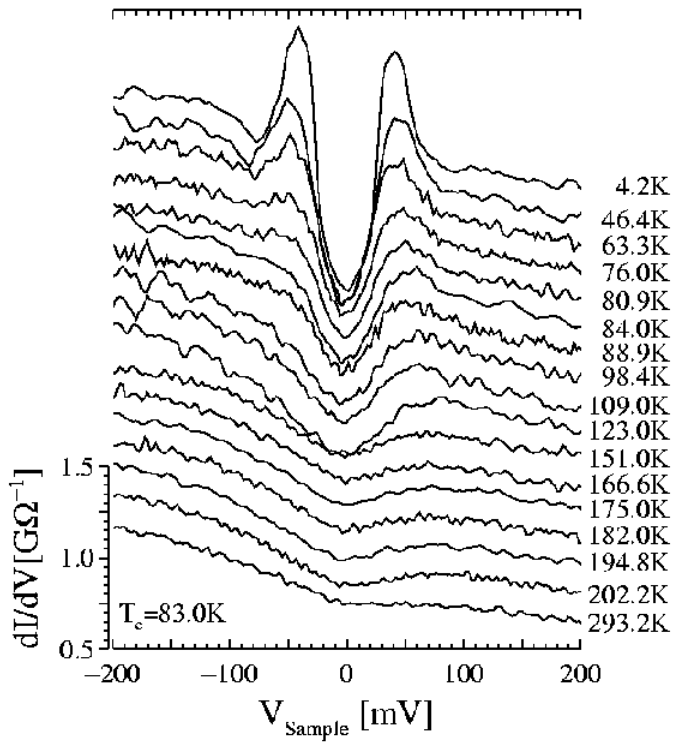

Fig. 2. Tunnelling density of states in a sample of underdoped $\mathrm{Bi}_{2} \mathrm{Sr}_{2} \mathrm{CaCu}_{2} \mathrm{O}_{8+\delta}$ $\left(T_{c}=83 \mathrm{~K}\right)$ as a function of temperature. Note that there is no tendency for the gap to close as $T_{c}$ is approached from below, but that the sharp "coherence peaks" in the spectrum do vanish at $T_{c}$. From Ref. 97

above $T_{c}$ as detected, primarily, in c-axis tunnelling 134 and ARPES 95, 96 experiments. The scale of energies and the momentum dependence of this suppression are very reminiscent of the $d$-wave superconducting gap observed in the same materials at temperatures well below $T_{c}$. This is highly suggestive of an identification between the pseudogap and some form of local superconducting pairing. Although a pseudogap energy scale is easily deduced from these experiments, it is not so clear to us that an unambiguous temperature scale can be cleanly obtained from them. (The c-axis here, and henceforth, refers to the direction perpendicular to the copper-oxide planes, which are also referred to, crystallographically, as the ab plane.)

2) $\mathbf{C u}$ NMR: There is a suppression of low energy spin fluctuations as detected 135] primarily in $\mathrm{Cu}$ NMR. In some cases, two rather different temperature scales are deduced from these experiments: an upper crossover temperature, at which a peak occurs in $\chi^{\prime}$, the real part of the uniform spin susceptibility (i.e. the Knight shift), and a lower crossover temperature,

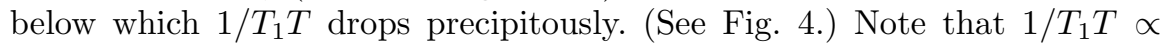
$\lim _{\omega \rightarrow 0} \int d \boldsymbol{k} f(\boldsymbol{k}) \chi^{\prime \prime}(\boldsymbol{k}, \omega) / \omega$, the $\boldsymbol{k}$ averaged density of states for magnetic excitations, where $f(\boldsymbol{k})$ is an appropriate form factor which reflects the local hyperfine coupling. Although the temperature scale deduced from $\chi^{\prime}$ is more or less in accordance with the pseudogap scale deduced from a number of 


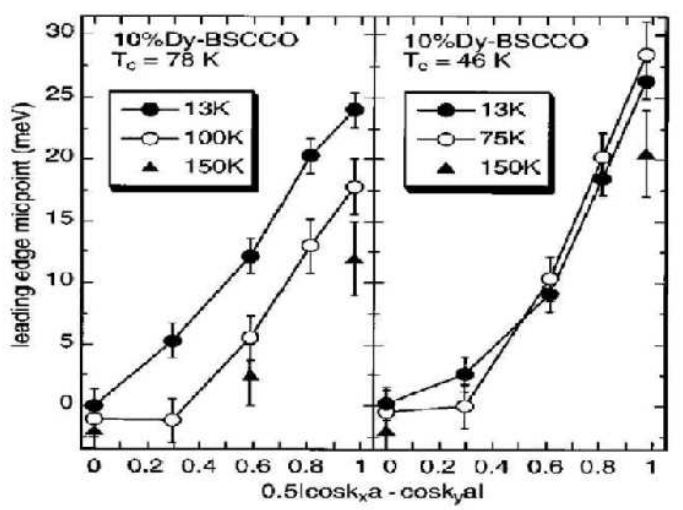

Fig. 3. The angular dependence of the gap in the normal and superconducting states of underdoped $\mathrm{Bi}_{2} \mathrm{Sr}_{2} \mathrm{Ca}_{1-x} \mathrm{Dy}_{x} \mathrm{Cu}_{2} \mathrm{O}_{8+\delta}$ as deduced from the leading edge energy of the single hole spectral function $A^{<}(\boldsymbol{k}, \omega)$ measured by ARPES. A straight line in this plot would correspond to the simplest $d_{x^{2}-y^{2}}$ gap, $\left|\Delta_{k}\right|=\Delta_{0} \mid \cos \left(k_{x}\right)-$ $\cos \left(k_{y}\right) \mid$. From Ref. 95.

other spectroscopies, it is actually a measure of the reactive response of the spin system. The notion of a gap can be more directly identified with a feature in $\chi^{\prime \prime}$. ( $A$ note of warning: while the structure in $1 / T_{1} T$ can be fairly sharp at times, the observed maxima in $\chi^{\prime}$ are always very broad and do not yield a sharply defined temperature scale without further analysis.)

3) Resistivity: There is a significant deviation [136, 137] of the resistivity in the ab plane from the $T$ linear temperature dependence which is universally observed at high temperatures. A pseudogap temperature is then identified as the point below which $d \rho_{x x} / d T$ deviates (increases) significantly from its high temperature value. (See Fig. 5.) In some cases, a similar temperature scale can be inferred from a scaling analysis of the Hall resistance, as well.

The pseudogap also appears in the c-axis resitivity, although in a somewhat different manner [138, 139]. In this direction, the pseudogap results in a strong increase in the resistivity, reminiscent of the behavior of a narrow gap semiconductor, as shown in Fig. 6. If we imagine that the c-axis transport is dominated by tunnelling events between neighboring planes, it is reasonable that a bulk measurement of $\rho_{c}$ will reflect the pseudogap in much the same way as the c-axis tunelling does.

4) Specific heat: There is a suppression of the expected electronic specific heat 82]. Above the pseudogap scale, the specific heat is generally found to be linear in temperature, $C_{V} \approx \gamma T$, but below the pseudogap temperature, $C_{V} / T$ begins to decrease with decreasing temperature. (See Fig. 7.) Interestingly, since the value of $\gamma$ above the pseudogap temperature appears to be roughly doping independent, the drop in the specific at 

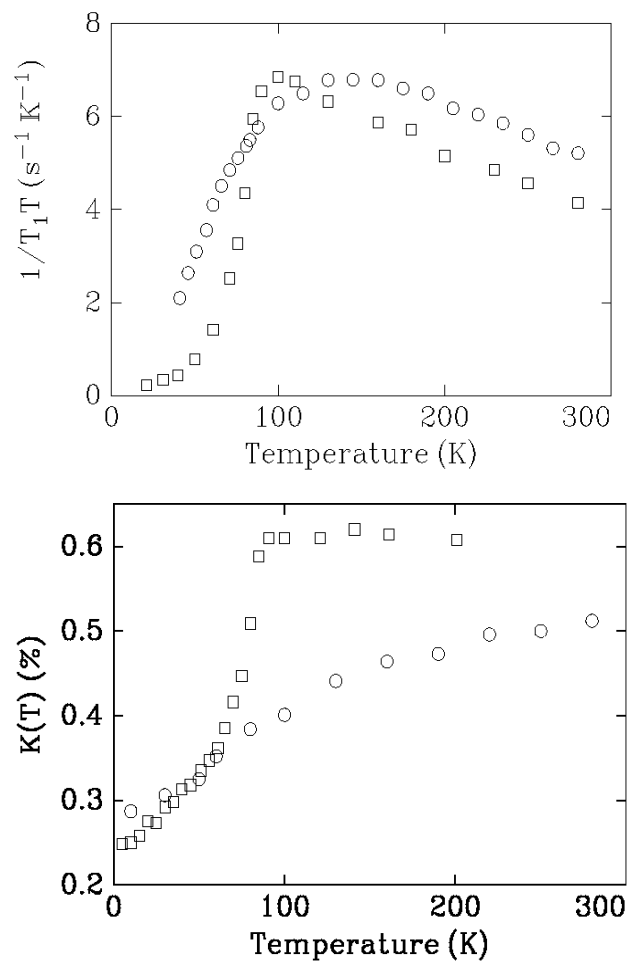

Fig. 4. Temperature dependence of the planar ${ }^{63} \mathrm{Cu}$ relaxation rate $1 / T_{1} T$ and Knight shift $K$ in optimally doped $\mathrm{YBa}_{2} \mathrm{Cu}_{3} \mathrm{O}_{6.95}$ (squares) and underdoped $\mathrm{YBa}_{2} \mathrm{Cu}_{3} \mathrm{O}_{6.64}$ (circles). From Ref. 81. 1

lower temperatures can be interpreted as a doping dependent loss of entropy, $\Delta S(x) \equiv S(x, T)-S\left(x_{\text {optimal }}, T\right)$, with a magnitude which is independent of temperature for any $T>T^{*}$. This is the origin of the famous (and still not understood) observation of Loram and collaborators 140] that there is a large entropy, $k_{B} / 2$, which is somehow associated with each doped hole. A word of warning: except at the lowest temperatures, the electronic specific heat is always a small fraction of the total specific heat, and complicated empirical subtraction procedures, for which the theoretical justification is not always clear to us, are necessary to extract the electronic contribution.

5) Infrared conductivity: There is an anomalous motion of infrared spectral weight to low energies [141, 142]. The pseudogap is most clearly identified by plotting 142 the frequency dependent scattering rate, defined either as $1 / \tau^{*}(\omega) \equiv \omega \sigma_{a b}^{\prime}(\omega) / \sigma_{a b}^{\prime \prime}(\omega)$, or as $1 / \tau(\omega)=\left[\omega_{P}^{2} / 4 \pi\right] \operatorname{Re}[1 / \sigma(\omega)]$ where $\omega_{P}$ is the plasma frequency; the pseudogap is rather harder to pick out from the in-plane conductivity, $\sigma_{a b}^{\prime}$, itself. At large $\omega$, one generally sees $1 / \tau(\omega) \approx A \omega$, 


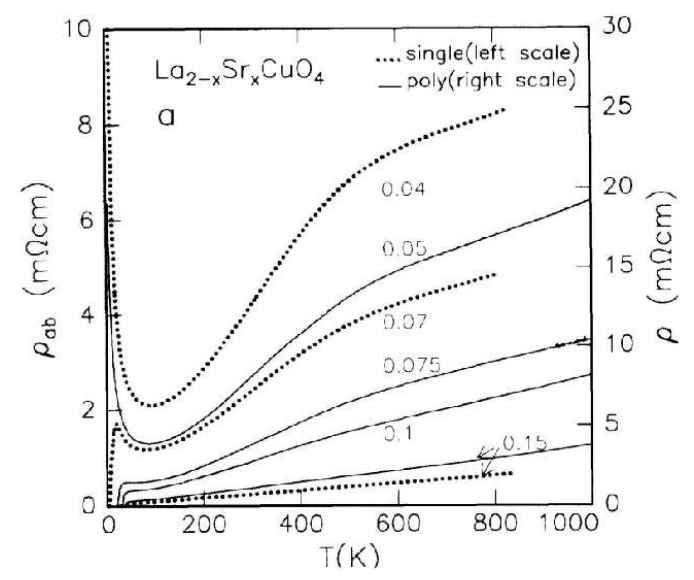

Fig. 5. The temperature dependence of the longitudinal resistivity in underdoped and optimally doped $\mathrm{La}_{2-x} \mathrm{Sr}_{x} \mathrm{CuO}_{4}$. The dotted lines correspond to the in-plane resistivity $\left(\rho_{a b}\right)$ of single crystal films while the solid lines depict the resistivity $(\rho)$ of polycrystalline samples. The doping levels are indicated next to the curves. From Ref. 136 .

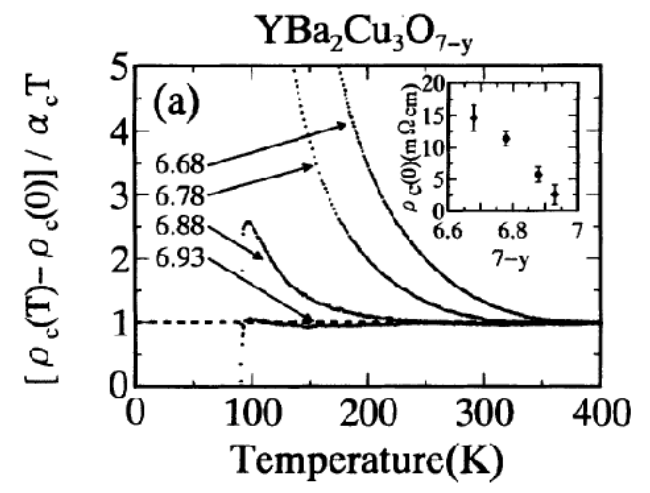

Fig. 6. The temperature dependence of the c-axis resistivity in underdoped and optimally doped $\mathrm{YBa}_{2} \mathrm{Cu}_{3} \mathrm{O}_{7-\delta}$. Here $\alpha_{c}$ and $\rho_{c}(0)$ are the slope and the intercept, respectively, when the metalic part of $\rho_{c}$ is approximated by a linear- $T$ behavior. The inset shows how $\rho_{c}(0)$ varies with oxygen content. From Ref. 138.

and it then drops to much smaller values, $1 / \tau \ll \omega$, below a characteristic pseudogap frequency, see Fig. 8. ( $A$ is generally a bit larger than 1 in underdoped materials and roughly equal to 1 in optimally doped ones.)

While in optimally doped materials, this manifestation of a pseudogap is only observed at temperatures less than $T_{c}$, in underdoped materials, it is seen to persist well above $T_{c}$, and indeed to be not strongly temperature dependent 


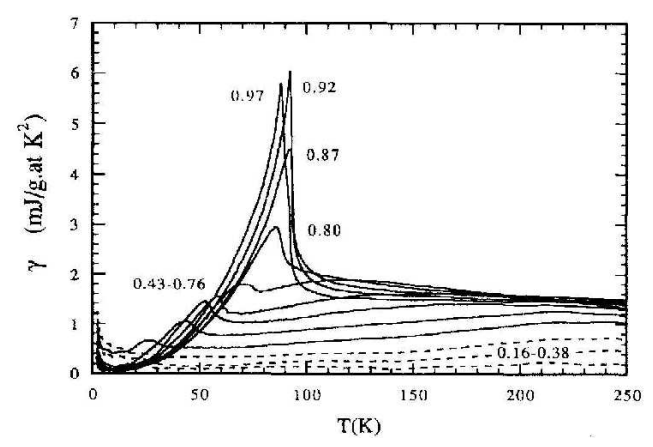

Fig. 7. Thermal density of "electronic" states, $\gamma \equiv C_{V} / T$ as a function of temperature for various oxygen concentrations in underdoped $\mathrm{YBa}_{2} \mathrm{Cu}_{3} \mathrm{O}_{6+x}$. From Ref. 140. As discussed in 140, a complicated proceedure has been used to subtract the large nonelectronic component of the measured specific heat.

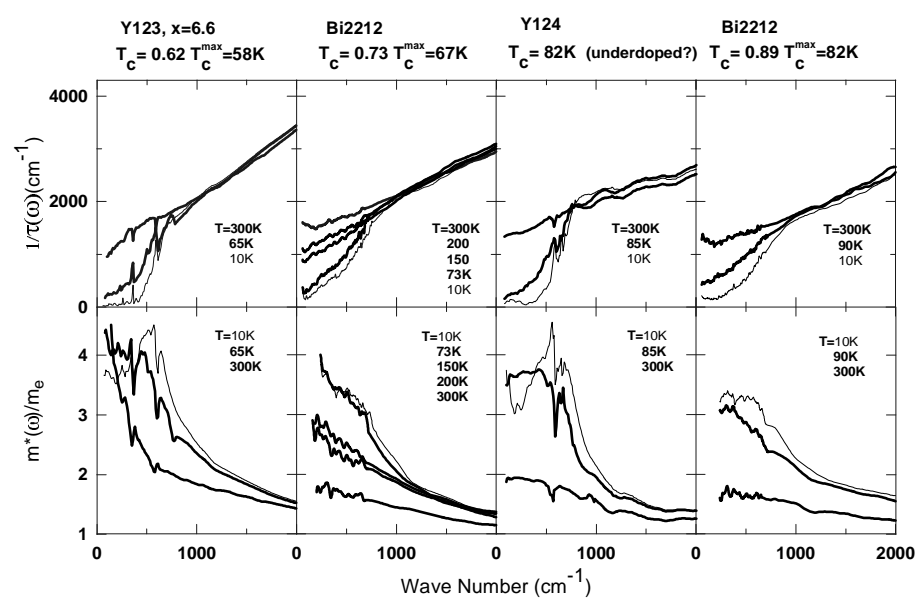

Fig. 8. Upper panels: Frequency dependent scattering rate for a series of underdoped cuprate superconductors above, near and below the superconducting transition temperature. Lower pannels: The effective mass enhancement $m^{*} / m_{e}=$ $1+\lambda(\omega)$. Both are deduced from fitting infrared conductivity data to an extended Drude model $\sigma=\left(\omega_{P}^{2} / 4 \pi\right) /[1 / \tau(\omega)-i \omega(1+\lambda(\omega))]$. From Ref. 142

near $T_{c}$. A characteristic pseudogap energy is easily identified from this data, but, again, it is not clear to us to what extent it is possible to identify a clear pseudogap temperature from this data. A pseudogap can also be deduced directly 143, 144 from an analysis of $\sigma_{c}^{\prime}(\omega)$, where it manifests itself as a suppressed response at low frequencies, as shown in Fig. 9 . 


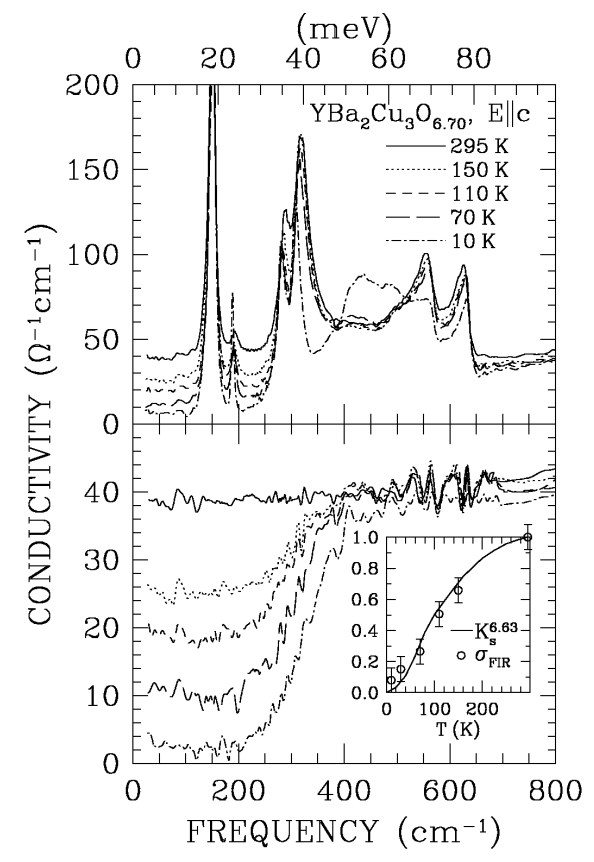

Fig. 9. The $c$-axis optical conductivity of underdoped $\mathrm{YBa}_{2} \mathrm{Cu}_{3} \mathrm{O}_{7-\delta}\left(T_{c}=63 \mathrm{~K}\right)$ as a function of temperature (top panel). The optical conductivity after the substraction of the phonon features is presented in the lower panel. The inset compares the low frequency conductivity with the Knight shift. From Ref. 143.

6) Inelastic neutron scattering: There are temperature dependent changes in the dynamic spin structure factor as measured by inelastic neutron scattering. Here, both features associated with low energy incommensurate magnetic correlations (possibly associated with stripes) 145] and the so-called "resonant peak" are found to emerge below a temperature which is very close to $T_{c}$ in optimally doped materials, but which rises considerably above $T_{c}$ in underdoped materials 146. (See Fig. 10.)

What does the pseudogap imply for theory? It is generally accepted that the pseudogap, in one way or another, reflects the collective physics associated with the growth of electronic correlations. This, more than any other aspect of the data, has focused attention on theories of the collective variables representing the order parameters of various possible broken symmetry states [20, 51 54, 62, 77, 147 158. Among these theories, there are two rather different classes of ways to interpret the pseudogap phenomena. 


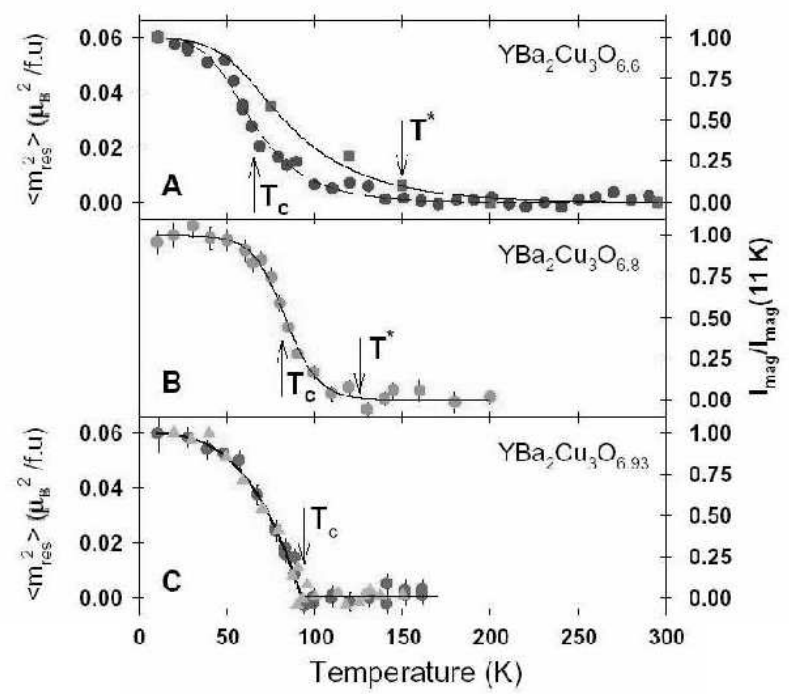

Fig. 10. The temperature dependence of the intensity of the so called resonant peak observed in neutron scattering in underdoped $\mathrm{YBa}_{2} \mathrm{Cu}_{3} \mathrm{O}_{7-\delta}$. From Ref. 146

1) It is well known that fluctuation effects can produce local order which, under appropriate circumstances, can extend well into the disordered phase. Such fluctuations produce in the disordered phase some of the local characteristics of the ordered phase, and if there is a gap in the ordered phase, a pseduogap as a fluctuation effect is eminently reasonable - see Fig. 1. As is discussed in Section 8, the small superfluid density of the cuprates leads to the unavoidable conclusion that superconducting fluctuations are an order 1 effect in these materials, so it is quite reasonable to associate some pseudogap phenomena with these fluctuations. However, as the system is progressively underdoped, it gets closer and closer to the antiferromagnetic insulating state, and indeed there is fairly direct NMR evidence of increasingly strong local antiferromagnetic correlations [159]. It is thus plausible that there are significant effects of antiferromagnetic fluctuations, and since the antiferromagnetic state also has a gap, one might expect these fluctuations to contribute to the pseudogap phenomena as well. There are significant incommensurate charge and spin density (stripe) fluctuations observed directly in scattering experiments on a variety of underdoped materials [47, 145, 160 162], as well as the occasional stripe ordered phase [163 167]. These fluctuations, too, certainly contribute to the observed pseudogap phenomena. Finally, fluctuations associated with more exotic phases, especially the "staggered flux phase" (which we will discuss momentarily) have been proposed [148, 168] as contributing to the pseudogap as well. 
There has been a tremendous amount of controversy in the literature concerning which of these various fluctuation effects best account for the observed pseudogap phenomena. Critical phenomena, which are clearly associated with the phase fluctuations of the superconducting order parameter, have been observed 169 172 in regions that extend between $10 \%$ to $40 \%$ above and below the superconducting $T_{c}$ in optimally and underdoped samples of $\mathrm{YBa}_{2} \mathrm{Cu}_{3} \mathrm{O}_{7-\delta}$ and $\mathrm{Bi}_{2} \mathrm{Sr}_{2} \mathrm{CaCu}_{2} \mathrm{O}_{8+\delta}$; in our opinion, the dominance of superconducting fluctuations in this substantial range of temperatures is now beyond question. However, pseudogap phenomena are clearly observed in a much larger range of temperatures. Even if fluctuation effects are ultimately the correct explanation for all the pseudogap phenomena, there may not truly be one type of fluctuation which dominates the physics over the entire range of temperatures.

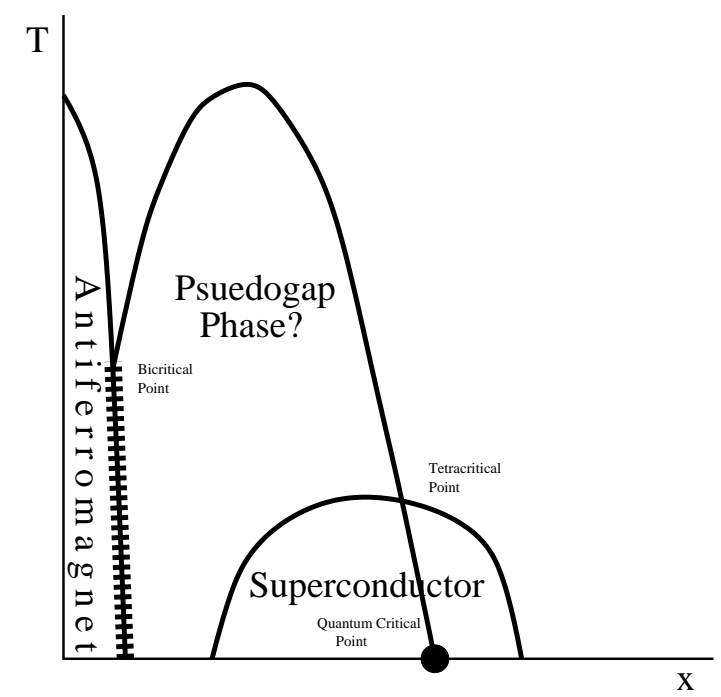

Fig. 11. There are many ideas concerning the meaning of the pseudogap. Defined purely phenomenologically, as shown in Fig. 1, it is a region in which there is a general reduction in the density of low energy excitations, and hence is bounded by an ill-defined crossover line. It is also possible that, to some extent, the pseudogap reflects the presence of a broken symmetry, in which case it must be bounded by a precise phase boundary, as shown in the present figure. There are many ways such a pseudogap phase could interact with the other well established phases. For purposes of illustration, we have shown a tetracritical and a bicritical point where the pseudogap meets, respectively, the superconducting and antiferromagnetic phases. One consequence of the assumption that the transition into the pseudogap phase is continuous is the exisence of a quantum critical point (indicated by the heavy circle) somewhere under the superconducting dome. See, for example, Refs. 20, 52, 54, 62, 173.

Crossovers can be murky. 
One cannot always tell a fluctuating superconductor from a fluctuating insulator!

Covert phase transitions are considered.
To illustrate this point explicitly, consider a one dimensional electron gas (at an incommensurate density) with weak attractive backscattering interactions. (See Section 5.) If the backscattering interactions are attractive $\left(g_{1}<0\right)$, they produce a spin gap $\Delta_{s}$. This gap persists as a pseudogap in the spectrum up to temperatures of order $\Delta_{s} / 2$. Now, because of the nature of fluctuations in one dimension, the system can never actually order at any finite temperature. However, there is a very real sense in which one can view the pseudogap as an effect of superconducting fluctuations, since at low temperatures, the superconducting susceptibility is proportional to $\Delta_{s}$. The problem is that one can equally well view the pseudogap as an effect of CDW fluctuations. One could arbitrarily declare that where the CDW susceptibility is the most divergent, the pseudogap should be viewed as an effect of local CDW order, while when the superconducting susceptibility is more divergent, it is an effect of local pairing. However, this position is untenable; by varying the strength of the forward scattering $\left(g_{2}\right)$, it is possible to pass smoothly from one regime to the other without changing $\Delta_{s}$ in any way!

2) There are several theoretical proposals [52 54] on the table which suggest that there is a heretofore undetected electronic phase transition in underdoped materials with a transition temperature well above the superconducting $T_{c}$. As a function of doping, this transition temperature is pictured as decreasing, and tending to zero at a quantum critical point somewhere in the neighborhood of optimal doping, as shown schematically in Fig. 11. If such a transition occurs, it would be natural to associate at least some of the observed pseudogap phenomena with it. Since these scenarios involve a new broken symmetry, they make predictions which are, in principle, sharply defined and falsifiable by experiment. However, there is an important piece of phenomenology which these theories must address: if there is a phase transition underlying pseudogap formation, why hasn't direct thermodynamic evidence (i.e. nonanalytic behavior of the specific heat, the susceptibility, or some other correlation function of the system) been seen in existing experiments? Possible answers to this question typically invoke disorder broadening of the proposed phase transition [54], rounding of the transition by a symmetry breaking field [52], or possibly the intrinsic weakness of the thermodynamic signatures of the transition under discussion [53, 174].

In any case, although these proposals are interesting in their own right, and potentially important for the interpretation of experiment, they are only indirectly related to the theory of high temperature superconductivity, which is our principal focus in this article. For this reason, we will not further pursue this discussion here.

\section{Preview: Our View of the Phase Diagram}

Clearly, the pseudogap phenomena described above are just the tip of the iceberg, and any understanding of the physics of the cuprate high temperature 
superconductors will necessarily be complicated. For this reason, we have arranged this article to focus primarily on high temperature superconducitivity as an abstract theoretical issue, and only really discuss how these ideas apply to the cuprates in Section 13. However, to orient the reader, we will take a moment here to briefly sketch our understanding of how these abstract issues determine the behavior, especially the high temperature superconductivity of the cuprates.

Fig. 12 is a schematic representation of the temperature vs. doping phase diagram of a representative cuprate. There are four energy scales relevant to the mechanism of superconductivity, marked as $T_{\text {stripe }}^{*}, T_{\text {pair }}^{*}, T_{3 D}^{*}$ and $T_{c}$. Away from the peak of the superconducting dome, these energy scales are often well separated. At least some of the pseudogap phenomena are, presumably, associated with the two crossover scales, $T_{\text {pair }}^{*}$ and $T_{\text {stripe }}^{*}$.

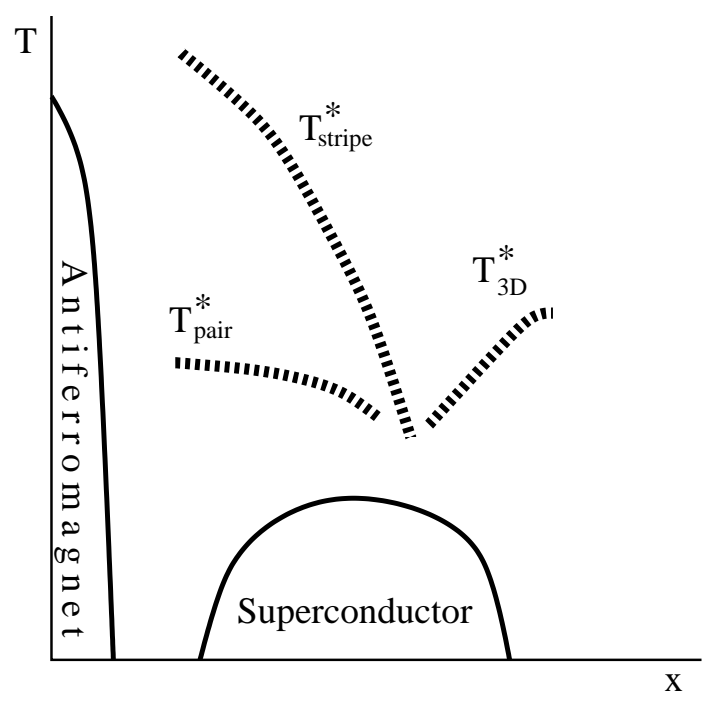

Fig. 12. Phase diagram as a function of temperature and doping within the stripes scenario discussed here.

Stripe Formation $T_{\text {stripe }}^{*}$ : The kinetic energy of doped holes is frustrated in an antiferromagnet. As the temperature is lowered through $T_{\text {stripe }}^{*}$, the doped holes are effectively ejected from the antiferromagnet to form metallic regions, thus relieving some of this frustration. Being charged objects, the holes can only phase separate on short length scales, since the Coulomb repulsion enforces charge homogeneity at long length scales. As a result, at $T_{\text {stripe }}^{*}$, the material develops significant one dimensional charge modulations, which we refer to as charge stripes. This can be an actual phase transition 
(e.g. to a "nematic phase"), or a crossover scale at which significant local charge stripe correlations develop.

Pair Formation $T_{\text {pair }}^{*}$ : While stripe formation permits hole delocalization in one direction, hole motion transverse to the stripe is still restricted. It is thus favorable, under appropriate circumstances, for the holes to pair so that the pairs can spread out somewhat into the antiferromagnetic neighborhood of the stripe. This "spin gap proximity effect" [20] (see Section 10.4], which is much like the proximity effect at the interface between a normal metal and a conventional superconductor, results in the opening of a spin gap and an enhancement of the superconducting susceptibility on a single stripe. In other words, $T_{\text {pair }}^{*}$ marks a crossover below which the superconducting order parameter amplitude (and therefore a superconducting pseudo gap) has developed, but without global phase coherence.

Superconductivity $T_{c}$ : Superconducting long ranged order onsets as the phase of the superconducting order parameter on each charge stripe becomes correlated across the sample. Since it is triggered by Josephson tunnelling between stripes, this is a kinetic energy driven phase ordering transition.

Dimensional Crossover $T_{3 D}^{*}$ : Superconducting long range order implies coherence in all three dimensions, and hence the existence of well defined electron-like quasiparticles [21, 149, 175]. Where the stripe order is sufficiently strong (in the underdoped regime), the dimensional crossover to 3D physics is directly associated with the onset of superconducting order. However, in overdoped materials, where the electron dynamics is less strongly influenced by stripe formation, we expect the dimensional crossover to occur well above $T_{c}$. (See Section 5.)

\section{Quasi-1D Superconductors}

In this section we address the physics of the one dimensional electron gas and quasi-one dimensional systems consisting of higher dimensional arrays of weakly coupled chains. Our motivation is twofold. Firstly, these systems offer a concrete realization of various non-Fermi liquid phenomena and are amenable to controlled theoretical treatments. As such they constitute a unique theoretical laboratory for studying strong correlations. In particular, for whatever reason,much of the experimentally observed behavior of the cuprate superconductors is strongly reminiscent [84, 86, 149] of a quasi-1D superconductor. Secondly, we are motivated by a growing body of experimental evidence for the existence of electron smectic and nematic phases in the high temperature superconductors, manganites and quantum Hall systems 6, 176 180. It is possible that these materials actually are quasi 1D on a local scale.

Our emphasis will be on quasi-one dimensional superconductors, the different unconventional signatures they exhibit as a function of temperature, and the conditions for their expression and stability. We will, however, in- 
clude some discussion of other quasi-one dimensional phases which typically tend to suppress superconductivity. It is also worth noting that, for the most part, the discussion is simply generalized to quasi-1D systems with different types of order, including quasi-1D CDW insulators.

\subsection{Elementary excitations of the 1DEG}

We begin by considering the continuum model of an interacting one dimensional electron gas (1DEG). It consists of approximating the 1DEG by a pair of linearly dispersing branches of left $(\eta=-1)$ and right $(\eta=1)$ moving spin $1 / 2(\sigma= \pm 1$ denotes the $z$ spin component) fermions constructed around the left and right Fermi points of the 1DEG. This approximation correctly describes the physics in the limit of low energy and long wavelength where the only important processes are those involving electrons in the vicinity of the Fermi points. The Hamiltonian density of the model is

$$
\begin{aligned}
\mathcal{H}= & -i v_{F} \sum_{\eta, \sigma= \pm 1} \eta \psi_{\eta, \sigma}^{\dagger} \partial_{x} \psi_{\eta, \sigma} \\
& +\frac{g_{4}}{2} \sum_{\eta, \sigma= \pm 1} \psi_{\eta, \sigma}^{\dagger} \psi_{\eta,-\sigma}^{\dagger} \psi_{\eta,-\sigma} \psi_{\eta, \sigma} \\
& +g_{2} \sum_{\sigma, \sigma^{\prime}= \pm 1} \psi_{1, \sigma}^{\dagger} \psi_{-1, \sigma^{\prime}}^{\dagger} \psi_{-1, \sigma^{\prime}} \psi_{1, \sigma} \\
& +g_{1 \|} \sum_{\sigma= \pm 1} \psi_{1, \sigma}^{\dagger} \psi_{-1, \sigma}^{\dagger} \psi_{1, \sigma} \psi_{-1, \sigma} \\
& +g_{1 \perp} \sum_{\sigma= \pm 1} \psi_{1, \sigma}^{\dagger} \psi_{-1,-\sigma}^{\dagger} \psi_{1,-\sigma} \psi_{-1, \sigma}
\end{aligned}
$$

where, e.g., $\psi_{1,1}$ destroys a right moving electron of spin $1 / 2$. The $g_{4}$ term describes forward scattering events of electrons in a single branch. The $g_{2}$ term corresponds to similar events but involving electrons on both branches. Finally, the $g_{1 \|}$ and $g_{1 \perp}$ terms allow for backscattering from one branch to the other. The system is invariant under $S U(2)$ spin rotations provided $g_{1 \|}=g_{1 \perp}=g_{1}$. In the following we consider mostly this case.

Umklapp processes of the form

$$
g_{3} \psi_{-1, \uparrow}^{\dagger} \psi_{-1, \downarrow}^{\dagger} \psi_{1, \downarrow} \psi_{1, \uparrow} e^{i\left(4 k_{F}-G\right) x}+\text { H.c. }
$$

are important only when $4 k_{F}$ equals a reciprocal lattice vector $G$. When the $1 \mathrm{DEG}$ is incommensurate $\left(4 k_{F} \neq G\right)$, the rapid phase oscillations in this term render it irrelevant in the renormalization group sense. We will assume such incommensurability and correspondingly ignore this term. We will also neglect single particle scattering between branches (for example due to disorder) and terms that do not conserve the $z$ component of the spin.

It is important to stress [181] that in considering this model we are focusing on the long distance physics that can be precisely derived from an effective 
Bosonization

field theory. However, all the coupling constants that appear in Eq. 8 are effective parameters which implicitly include much of the high energy physics. For instance, the bare velocity which enters the model, $v_{F}$, is not necessarily simply related to the dispersion of the band electrons in a zeroth order, noninteracting model, but instead includes all sorts of finite renormalizations due to the interactions. The weak coupling perturbative renormalization group treatment of this model is discussed in Section 9, below; the most important result from this analysis is that the Fermi liquid fixed point is always unstable, so that an entirely new, nonperturbative method must be employed to reveal the low energy physics.

Fortunately, such a solution is possible; the Hamiltonian in Eq. (8) is equivalent to a model of two independent bosonic fields, one representing the charge and the other the spin degrees of freedom in the system. (For reviews and recent perspectives see Refs. 38, 181 187.) The two representations are related via the bosonization identity

$$
\psi_{\eta, \sigma}=\frac{1}{\sqrt{2 \pi a}} F_{\eta, \sigma} \exp \left[-i \Phi_{\eta, \sigma}(x)\right]
$$

which expresses the fermionic fields in terms of self dual fields $\Phi_{\eta, \sigma}(x)$ obeying $\left[\Phi_{\eta, \sigma}(x), \Phi_{\eta^{\prime}, \sigma^{\prime}}\left(x^{\prime}\right)\right]=-i \pi \delta_{\eta, \eta^{\prime}} \delta_{\sigma, \sigma^{\prime}} \operatorname{sign}\left(x-x^{\prime}\right)$. They in turn are combinations of the bosonic fields $\phi_{c}$ and $\phi_{s}$ and their conjugate momenta $\partial_{x} \theta_{c}$ and $\partial_{x} \theta_{s}$

$$
\Phi_{\eta, \sigma}=\sqrt{\pi / 2}\left[\left(\theta_{c}-\eta \phi_{c}\right)+\sigma\left(\theta_{s}-\eta \phi_{s}\right)\right] .
$$

Physically, $\phi_{c}$ and $\phi_{s}$ are, respectively, the phases of the $2 k_{F}$ charge density wave (CDW) and spin density wave (SDW) fluctuations, and $\theta_{c}$ is the superconducting phase. In terms of them the long wavelength component of the charge and spin densities are given by

$$
\begin{aligned}
\rho(x) & =\sum_{\eta, \sigma} \psi_{\eta, \sigma}^{\dagger} \psi_{\eta, \sigma}-\frac{2 k_{F}}{\pi}=\sqrt{\frac{2}{\pi}} \partial_{x} \phi_{c}, \\
S_{z}(x) & =\frac{1}{2} \sum_{\eta, \sigma} \sigma \psi_{\eta, \sigma}^{\dagger} \psi_{\eta, \sigma}=\sqrt{\frac{1}{2 \pi}} \partial_{x} \phi_{s} .
\end{aligned}
$$

The Klein factors $F_{\eta, \sigma}$ in Eq. (9) are responsible for reproducing the correct anticommutation relations between different fermionic species and $a$ is a short distance cutoff that is taken to zero at the end of the calculation.

The widely discussed separation of charge and spin in this problem is In $1 D$ spin and formally a statement that the Hamiltonian density can be expressed as a charge separate. sum of two pieces, each of the sine-Gordon variety, involving only charge or spin fields

$$
\mathcal{H}=\sum_{\alpha=c, s}\left\{\frac{v_{\alpha}}{2}\left[K_{\alpha}\left(\partial_{x} \theta_{\alpha}\right)^{2}+\frac{\left(\partial_{x} \phi_{\alpha}\right)^{2}}{K_{\alpha}}\right]+V_{\alpha} \cos \left(\sqrt{8 \pi} \phi_{\alpha}\right)\right\}
$$


When the Hamiltonian is separable, wavefunctions, and therefore correlation functions, factor. (See Eqs. 24) and 25).) In terms of the parameters of the fermionic formulation Eq. (8) the charge and spin velocities are given by

$$
\begin{aligned}
& v_{c}=\frac{1}{2 \pi} \sqrt{\left(2 \pi v_{F}+g_{4}\right)^{2}-\left(g_{1 \|}-2 g_{2}\right)^{2}}, \\
& v_{s}=\frac{1}{2 \pi} \sqrt{\left(2 \pi v_{F}-g_{4}\right)^{2}-g_{1 \|}^{2}},
\end{aligned}
$$

while the Luttinger parameters $K_{\alpha}$, which determine the power law behavior of the correlation functions, are

$$
\begin{aligned}
K_{c} & =\sqrt{\frac{2 \pi v_{F}+g_{4}-2 g_{2}+g_{1 \|}}{2 \pi v_{F}+g_{4}+2 g_{2}-g_{1 \|}}}, \\
K_{s} & =\sqrt{\frac{2 \pi v_{F}-g_{4}+g_{1 \|}}{2 \pi v_{F}-g_{4}-g_{1 \|}}} .
\end{aligned}
$$

The cosine term in the spin sector of the bosonized version of the Hamiltonian (Eq. (13)) originates from the back scattering term in Eq. (8) where the amplitudes are related according to

$$
V_{s}=\frac{g_{1 \perp}}{2(\pi a)^{2}} .
$$

The corresponding term in the charge sector describes umklapp processes and in view of our assumption will be set to zero $V_{c}=0$. Eqs. (14 18) complete the exact mapping between the fermionic and bosonic field theories.

In the absence of back scattering $\left(g_{1}=0\right)$ this model is usually called the Tomonaga-Luttinger model. Since $\partial_{x} \theta_{c, s}$ and $\phi_{c, s}$ are canonically conjugate, it is clear from the form of the bosonized Hamiltonian (Eq. (13)) that it describes a collection of independent charge and spin density waves with linear dispersion $\omega_{c, s}=v_{c, s} k$. The quadratic nature of the theory and the coherent representation (Eq. (9)) of the electronic operators in terms of the bosonic fields allow for a straightforward evaluation of various electronic correlation functions.

For $g_{1} \neq 0$ the spin sector of the theory turns into a sine-Gordon theory whose renormalization group flow is well known [188]. In particular, for repulsive interactions $\left(g_{1}>0\right)$ the backscattering amplitude is renormalized to zero in the long wavelength low energy limit and consequently at the fixed point $K_{s}=1$. On the other hand, in the presence of attractive interactions $\left(g_{1}<0\right)$ the model flows to strong (negative) coupling where the cosine term in Eq. (13) is relevant. As a result $\phi_{s}$ is pinned in the sense that in the ground state, it executes only small amplitude fluctuations about its classical ground state value (i.e. one of the minima of the cosine). There is a spin gap to both extended phonon-like small amplitude oscillations about this minimum and 
large amplitude soliton excitations that are domain walls at which $\phi_{s}$ changes between two adjacent minima.

The susceptibility of the interacting one dimensional electron gas to various instabilities can be investigated by calculating the correlation functions of the operators that describe its possible orders. They include, among others, the $2 k_{F}$ CDW and SDW operators

$$
\begin{aligned}
O_{C D W}(x) & =e^{-i 2 k_{F} x} \sum_{\tau} \psi_{1, \tau}^{\dagger}(x) \psi_{-1, \tau}(x), \\
O_{S D W_{\alpha}}(x) & =e^{-i 2 k_{F} x} \sum_{\tau, \tau^{\prime}} \psi_{1, \tau}^{\dagger}(x) \sigma_{\tau, \tau^{\prime}}^{\alpha} \psi_{-1, \tau^{\prime}}(x),
\end{aligned}
$$

where $\boldsymbol{\sigma}$ are the Pauli matrices, the $4 k_{F}$ CDW (or Wigner crystal) order

$$
O_{4 k_{F}}(x)=e^{-i 4 k_{F} x} \sum_{\tau} \psi_{1, \tau}^{\dagger}(x) \psi_{1,-\tau}^{\dagger}(x) \psi_{-1,-\tau}(x) \psi_{-1, \tau}(x),
$$

and the singlet (SS) and triplet (TS) pair annihilation operators

$$
\begin{aligned}
O_{S S}(x) & =\sum_{\tau} \tau \psi_{1, \tau}(x) \psi_{-1,-\tau}(x), \\
O_{T S_{\alpha}}(x) & =\sum_{\tau, \tau^{\prime}} \tau \psi_{1, \tau}(x) \sigma_{\tau, \tau^{\prime}}^{\alpha} \psi_{-1,-\tau^{\prime}}(x) .
\end{aligned}
$$

They can also be written in a suggestive bosonized form. For example the CDW and the singlet pairing operators are expressed as ${ }^{12}$

$$
\begin{aligned}
O_{C D W}(x) & =\frac{e^{-2 i k_{F} x}}{\pi a} \cos \left[\sqrt{2 \pi} \phi_{s}(x)\right] e^{-i \sqrt{2 \pi} \phi_{c}(x)}, \\
O_{S S}(x) & =\frac{1}{\pi a} \cos \left[\sqrt{2 \pi} \phi_{s}(x)\right] e^{-i \sqrt{2 \pi} \theta_{c}(x)} .
\end{aligned}
$$

$1 D$ order parameters have "spin" amplitudes and "charge" phases.
The distinct roles of spin and charge are vividly apparent in these expressions: the amplitude of the order parameters is a function of the spin fields while their phase is determined by the charge degrees of freedom. Similar relations are found for the SDW and triplet pairing operators. However, the $4 k_{F}$ CDW order is independent of the spin fields.

If in the bare Hamiltonian, $g_{1}>0$ and $V_{s}$ is not too large, the system flows to the Gaussian fixed point with $K_{s}=1$ and no spin gap. The gapless fluctuations of the amplitude (spin) and phase (charge) of the various orders lead then to an algebraic decay of their zero temperature space-time correlation functions (with logarithmic corrections which reflect the slow renormalization of marginally irrelevant operators near the fixed point [189]):

$$
\left\langle O_{C D W}^{\dagger}(x) O_{C D W}(0)\right\rangle \propto e^{2 i k_{F} x} x^{-\left(1+K_{c}\right)} \ln ^{-3 / 2}(x),
$$

\footnotetext{
${ }^{12}$ For a discussion of some delicate points involving Klein factors in such expressions see Refs. 184 and 186.
} 


$$
\begin{aligned}
\left\langle O_{S D W_{\alpha}}^{\dagger}(x) O_{S D W_{\alpha}}(0)\right\rangle & \propto e^{2 i k_{F} x} x^{-\left(1+K_{c}\right)} \ln ^{1 / 2}(x), \\
\left\langle O_{4 k_{F}}^{\dagger}(x) O_{4 k_{F}}(0)\right\rangle & \propto e^{4 i k_{F} x} x^{-4 K_{c}} \\
\left\langle O_{S S}^{\dagger}(x) O_{S S}(0)\right\rangle & \propto x^{-\left(1+1 / K_{c}\right)} \ln ^{-3 / 2}(x), \\
\left\langle O_{T S_{\alpha}}^{\dagger}(x) O_{T S_{\alpha}}(0)\right\rangle & \propto x^{-\left(1+1 / K_{c}\right)} \ln ^{1 / 2}(x),
\end{aligned}
$$

where the proportionality involves model dependent constants and where subleading terms have been omitted. In the presence of interactions that break spin rotation symmetry $\left(g_{1 \|} \neq g_{1 \perp}\right)$ the model flows, for moderately repulsive bare $g_{1 \|}$, to a point on a fixed line with $V_{s}=0$ and $K_{s}>1$. Correspondingly, the spin contribution to the decay exponent of the correlation functions (see Eq. (26)) changes from 1 to $K_{s}$ for the CDW, SS, and the z component of the SDW order, and from 1 to $1 / K_{s}$ for TS and the $\mathrm{x}$ and y components of the SDW order. (For $K_{s} \neq 1$, there are no logarithmic corrections and the leading behavior is that of a pure power law [189.)

The temporal dependence of the above correlation functions is easily obtained owing to the Lorentz invariance of the model (Eq. (13)). By Fourier transforming them one obtains the related susceptibilities whose low temperature behavior for the spin rotationally invariant case is given according to

$$
\begin{aligned}
\chi_{C D W} & \propto T^{K_{c}-1}|\ln (T)|^{-3 / 2}, \\
\chi_{S D W} & \propto T^{K_{c}-1}|\ln (T)|^{1 / 2}, \\
\chi_{4 k_{F}} & \propto T^{4 K_{c}-2}, \\
\chi_{S S} & \propto T^{1 / K_{c}-1}|\ln (T)|^{-3 / 2}, \\
\chi_{T S} & \propto T^{1 / K_{c}-1}|\ln (T)|^{1 / 2} .
\end{aligned}
$$

Therefore in the absence of a spin gap and for $1 / 3<K_{c}<1$, the $2 k_{F}$ fluctuations are the most divergent, and the SDW is slightly more divergent than the CDW. In the presence of strong repulsive interactions when $K_{c}<$ $1 / 3$, the $4 k_{F}$ correlations dominate. If $K_{c}>1$, the pairing susceptibilities diverge at low temperatures and triplet pairing is the dominant channel.

When $g_{1}<0$, a spin gap opens of magnitude

$$
\Delta_{s} \sim \frac{v_{s}}{a}\left(\frac{\left|g_{1}\right|}{2 \pi v_{s}}\right)^{1 /\left(2-2 K_{s}\right)} .
$$

This can be explicitly demonstrated at the special Luther-Emery point 190 $K_{s}=1 / 2$, where the spin sector is equivalent to a massive free Dirac theory. At this point, a new set of spinless fermions can be defined

$$
\Psi_{\eta} \equiv \frac{1}{\sqrt{2 \pi a}} F_{\eta} \exp \left[i \sqrt{\pi / 2}\left(\theta_{s}-2 \eta \phi_{s}\right)\right]
$$

in terms of which the spin part of the Hamiltonian can be refermionized

$$
\mathcal{H}_{s}=-i v_{s} \sum_{\eta} \eta \Psi_{\eta}^{\dagger} \partial_{x} \Psi_{\eta}+\Delta_{s}\left(\Psi_{1}^{\dagger} \Psi_{-1}+\text { H.c. }\right),
$$

Without a spin gap, SDW and triplet pairing fluctuations are most relevant. 
and readily diagonalized to obtain the spin excitation spectrum

$$
E_{s}=\sqrt{v_{s}^{2} k^{2}+\Delta_{s}^{2}} .
$$

With a spin gap, $C D W$ or singlet pairing fluctuations are the most relevant.
In the spin gapped phase, correlations involving spin 1 order parameters, such as SDW and triplet pairing, decay exponentially with correlation length $\xi_{s}=v_{s} / \Delta_{s}$. On the other hand the amplitude of the CDW and SS order parameters acquire a vacuum expectation value. Actual long range order, however, does not occur due to the phase fluctuations associated with the still gapless charge modes. Nevertheless, the CDW and SS susceptibilities are enhanced compared to the case with no spin gap and in a spin rotationally invariant system are given by

$$
\begin{aligned}
\chi_{C D W} & \propto \Delta_{s} T^{K_{c}-2}, \\
\chi_{S S} & \propto \Delta_{s} T^{1 / K_{c}-2} .
\end{aligned}
$$

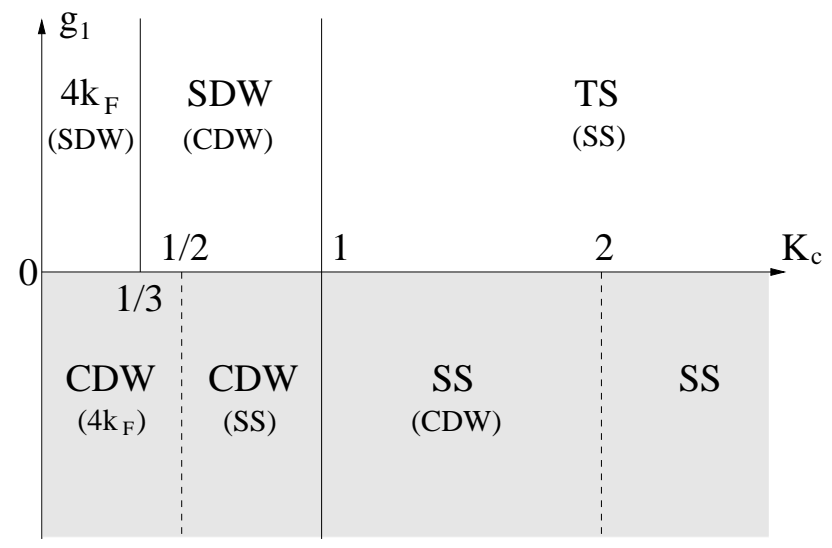

Fig. 13. Phase diagram for the one dimensional spin rotationally invariant electron gas showing where various zero temperature correlations diverge. Parentheses indicate subdivergent correlations and the shaded region contains the spin gapped phases. The order parameters that appear in the figure are: singlet superconductivity (SS); triplet superconductivity (TS); $2 k_{F}$ spin density wave (SDW); $2 k_{F}$ charge density wave $(\mathrm{CDW})$; and $4 k_{F}$ charge density wave $\left(4 \mathrm{k}_{\mathrm{F}}\right)$.

As long as $K_{c}>1 / 2$ the singlet pairing susceptibility is divergent but it becomes more divergent than the CDW susceptibility only when $K_{c}>1$. The latter diverges for $K_{c}<2$ and is the predominant channel provided $K_{c}<1$. Figure 13 summarizes the situation for low temperatures showing where in parameter space each type of correlation diverges. 
Concerning the sign of the effective interactions.
We see that the low energy behavior of a system with a spin gap is basically determined by a single parameter $K_{c}$. For a Hubbard chain with repulsive interactions, it is well known [191] that $K_{c}<1$, but this is not a general physical bound. For instance, numerical experiments on two leg Hubbard ladders (which are spin gapped systems as we discuss in Sections 10 and 11) have found a power law decay $r^{-\theta}$ of the singlet $d$-wave pairing correlations along the ladder. Fig. 14 presents the minimal value of the decay exponent $\theta$ obtained for ladders with varying ratio of inter- to intra-leg hopping $t_{\perp} / t$ as a function of the relative interaction strength $U / t$ [192]. By comparing it with the corresponding exponent $\theta=1 / K_{c}$ calculated for a spin gapped one dimensional system, one can see that $K_{c}>1 / 2$ over the entire range of parameters and that for some ranges $K_{c}>1$. Our point is that in a multicomponent 1DEG, it is possible to have $K_{c}>1$ (and thus singlet superconductivity as the most divergent susceptibility) even for repulsive interactions.

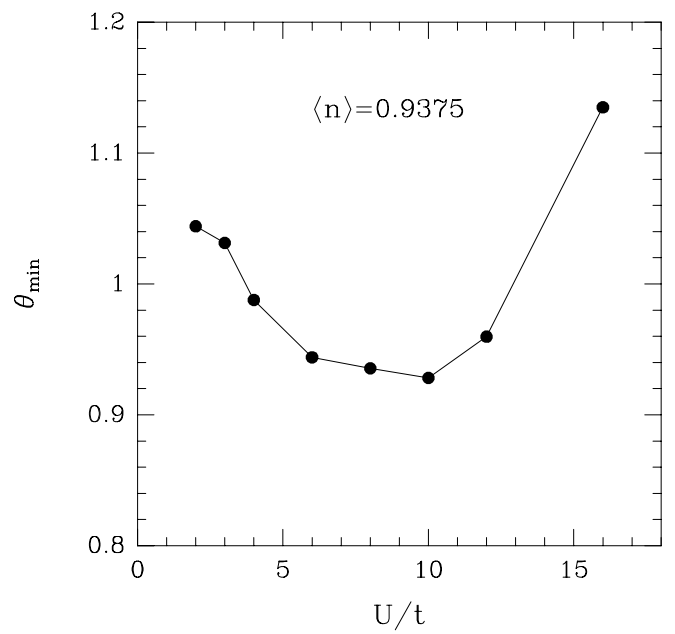

Fig. 14. Minimal value of the decay exponent, $\theta=1 / K_{c}$, of the $d$-wave singlet pairing correlations in a two leg ladder with varying hopping ratio $t_{\perp} / t$ as a function of $U / t$. The electron filling is $\langle n\rangle=0.9375$. (From Noack et al. 192])

\subsection{Spectral functions of the 1DEG - signatures of fractionalization}

The fact that one can obtain a strong (power law) divergence of the superconducting susceptibility from repulsive interactions between electrons is certainly reason enough to look to the 1DEG for clues concerning the origins of high temperature superconductivity — we will further pursue this in 
Sections 10 and 11, below. What we will do now is to continue to study the 1DEG as a solved model of a non-Fermi liquid.

In a Fermi liquid the elementary excitations have the quantum numbers of an electron and a nonvanishing overlap with the state created by the electronic creation operator acting on the ground state. As a result the single particle spectral function, $A(\boldsymbol{k}, \omega)$, is peaked at $\omega=\epsilon(\boldsymbol{k})=\boldsymbol{v}_{F}\left(\boldsymbol{k}_{F}\right) \cdot\left(\boldsymbol{k}-\boldsymbol{k}_{F}\right)$, where $\epsilon(\boldsymbol{k})$ is the quasiparticle dispersion relation. This peak can be and has been [193] directly observed using angle resolved photoemission spectroscopy (ARPES) which measures the single hole piece of the spectral function

$$
A^{<}(\boldsymbol{k}, \omega)=\int_{-\infty}^{\infty} d \boldsymbol{r} d t e^{i(\boldsymbol{k} \cdot \boldsymbol{r}+\omega t)}\left\langle\psi_{\sigma}^{\dagger}(\boldsymbol{r}, t) \psi_{\sigma}(0,0)\right\rangle .
$$

The lifetime of the quasiparticle, $\tau(\boldsymbol{k})$, can be determined from the width of the peak in the "energy distribution curve" (EDC) defined by considering $A^{<}(\boldsymbol{k}, \omega)$ at fixed $\boldsymbol{k}$ as a function of $\omega$ :

$$
1 / \tau=\Delta \omega .
$$

In a Fermi liquid, so long as the quasiparticle excitation is well defined (i.e. the decay rate is small compared to the binding energy) this width is related via the Fermi velocity to the peak width $\Delta k$ in the "momentum distribution curve" (MDC). This curve is defined as a cross section of $A^{<}(\boldsymbol{k}, \omega)$ taken at constant binding energy, $\omega$. Explicitly

$$
\Delta \omega=v_{F} \Delta k .
$$

There are no stable excitations of the $1 D E G$ with quantum numbers of an electron.
A very different situation occurs in the theory of the 1DEG where the elementary excitations, charge and spin density waves, do not have the quantum numbers of a hole. Despite the fact that the elementary excitations are bosons, they give rise to a linear in $T$ specific heat that is not qualitatively different from that of a Fermi liquid. However, because of the separation of charge and spin, the creation of a hole (or an electron) necessarily implies the creation of two or more elementary excitations, of which one or more carries its spin and one or more carries its charge. Consequently, $A^{<}(k, \omega)$ does not have a pole contribution, but rather consists of a multiparticle continuum which is distributed over a wide region in the $(k, \omega)$ plane. The shape of this region is determined predominantly by the kinematics. The energy and momentum of an added electron are distributed between the constituent charge and spin pieces. In the case where both of them are gapless [see Figs. 15.(a) and 15(b)] this means

$$
\begin{aligned}
E & =v_{c}\left|k_{c}\right|+v_{s}\left|k_{s}\right|, \\
k & =k_{c}+k_{s},
\end{aligned}
$$

where energy and momentum are measured relative to $E_{F}$ and $k_{F}$ respectively. Consequently any point above the dispersion curve of the slower excitation (taken here to be the spin) may be reached by placing an appropriate 
(a)

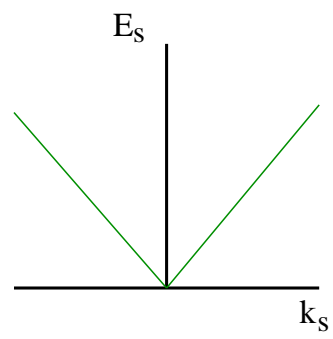

(c)

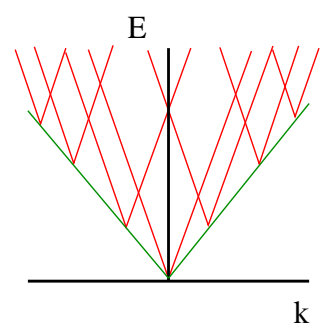

(b)

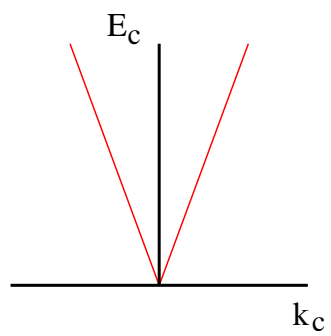

(d)

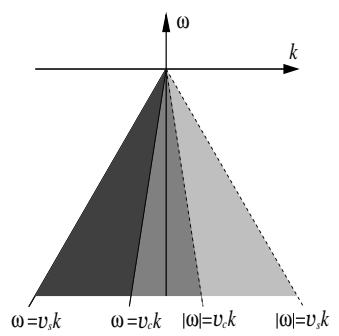

Fig. 15. Kinematics of the 1DEG: (a) Dispersion of the spin excitations. (b) Dispersion of the charge excitations. (c) The available electronic states. (d) Kinematic constraints on the spectral function: $A^{<}(k, \omega)$ for the 1DEG is nonzero at $T=0$ only in the shaded region of the $(k, \omega)$ plane. In the spin rotationally invariant case, $K_{s}=1, A^{<}(k, \omega)=0$ in the lightly shaded region, as well. If in addition, $K_{c}=1$, $A^{<}(k, \omega)=0$ outside of the darkest region. We have assumed $v_{c}>v_{s}$, which is usually the case in realistic systems.

amount of energy and momentum into the spin degrees of freedom, and the remaining energy and momentum into the charge degrees of freedom, as shown in Fig. 15(c). The addition of a hole is similarly constrained kinematically, and the corresponding zero temperature ARPES response has weight only within the shaded regions of Fig. 15(d).

Further constraints on the distribution of spectral weight may arise from symmetries. In the spin rotationally invariant case, at the fixed point $K_{s}=1$, the spin correlators do not mix left and right moving pieces. As a consequence, $A^{<}(T=0)$ for a right moving hole vanishes when $\omega$ is in the range $v_{s} k \leq$ $|\omega| \leq v_{c} k$ (assuming $v_{s}<v_{c}$ and $k>0$ ), even if the kinematic conditions are satisfied; See Fig. 15(d). ${ }^{13}$ If in addition $K_{c}=1$, so that the charge piece also does not mix left and right movers, $A^{<}(T=0)$ vanishes unless $k<0$ and $v_{s}|k| \leq|\omega| \leq v_{c}|k|$, (the darkest region in Fig. 15(d)). While $K_{s}=1$ is

${ }^{13}$ While the kinematic constraints are symmetric under $k \rightarrow-k$, the dynamical considerations are not, since although we have shifted the origin of $k$, we are in fact considering a right moving electron, i.e. one with momentum near $+k_{F}$. 
fixed by symmetry, there is no reason why $K_{c}$ should be precisely equal to 1. However, if the interactions are weak, (i.e. if $K_{c}$ is near 1) most of the spectral weight is still concentrated in this region. It spreads throughout the rest of the triangle with increasing interaction strength.

Clearly, the total width of the MDC is bounded by kinematics and is

A dichotomy between sharp $M D C$ 's and broad EDC's is a telltale sign of electron fractionalization. at most $\Delta k_{\max }=2|\omega| / \min \left(v_{c}, v_{s}\right)$. Any peak in the MDC will have a width which equals a fraction of this, depending on the interactions and symmetries of the problem, but in any case will vanish as the Fermi energy is approached. By contrast, at $k=0$, the shape of the EDC is not given by the kinematics at all, but is rather determined by the details of the matrix elements linking the one hole state to the various multi particle-hole states which form the continuum. In this case, the spectrum has a nonuniversal power law behavior with exponents determined by the interactions in the 1DEG. Whenever such a dichotomy between the MDC and EDC is present, it can be taken as evidence of electron fractionalization 86].

These general considerations can be substantiated by examining the explicit expression for the spectral function of the Tomonaga-Luttinger model [194 197. The quantum criticality and the spin-charge separation of the model imply a scaling form for its correlation functions

$$
A^{<}(k, \omega) \propto T^{2\left(\gamma_{c}+\gamma_{s}\right)+1} \int d q d \nu G_{c}(q, \nu) G_{s}(\tilde{k}-r q, \tilde{\omega}-\nu)
$$

where we introduce the velocity ratio $r=v_{s} / v_{c}$ and define the scaling variables

$$
\tilde{k}=\frac{v_{s} k}{\pi T} \quad, \quad \tilde{\omega}=\frac{\omega}{\pi T} .
$$

Since the spin and charge sectors are formally invariant under separate Lorentz transformations, the functions $G_{\alpha},(\alpha=c, s)$ also split into right and left moving parts

$$
G_{\alpha}(k, \omega)=\frac{1}{2} h_{\gamma_{\alpha}+\frac{1}{2}}\left(\frac{\omega+k}{2}\right) h_{\gamma_{\alpha}}\left(\frac{\omega-k}{2}\right),
$$

where $h_{\gamma}$ is expressed via the beta function

$$
h_{\gamma}(k)=\operatorname{Re}\left[(2 i)^{\gamma} B\left(\frac{\gamma-i k}{2}, 1-\gamma\right)\right]
$$

and the exponents

$$
\gamma_{\alpha}=\frac{1}{8}\left(K_{\alpha}+K_{\alpha}^{-1}-2\right)
$$

are defined so that $\gamma_{\alpha}=0$ for noninteracting fermions.

Fig. 16 depicts MDC's at the Fermi energy $(\omega=0)$ and EDC's at the Fermi wavevector $(k=0)$ for a spin rotationally invariant $\left(\gamma_{s}=0\right)$ Tomonaga-Luttinger model for various values of the parameter $\gamma_{c}$. While the 

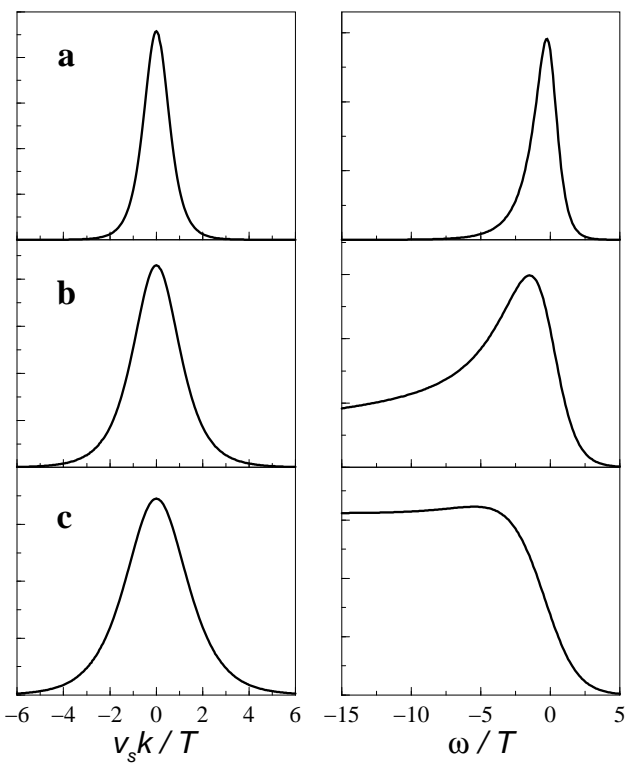

Fig. 16. MDC's at $\omega=0$ (left) and EDC's at $k=0$ (right), for a spin rotationally invariant Tomonaga-Luttinger liquid, with $v_{c} / v_{s}=3$ and a) $\left.\gamma_{c}=0, \mathrm{~b}\right) \gamma_{c}=0.25$, and c) $\gamma_{c}=0.5$.

MDC's broaden somewhat with increasing interaction strength they remain relatively sharp with a well defined peak structure. On the other hand any corresponding structure in the EDC's is completely wiped out in the presence of strong interactions. Such behavior has been observed in ARPES studies of quasi-one dimensional compounds as depicted in Fig. 17 as well as in the cuprate high temperature superconductors [86].

Away from the Fermi energy and Fermi wavevector and for not too strong interactions the peaks in the MDC and EDC split into a double peak structure, one dispersing with $v_{s}$ and the other with $v_{c}$. If observed this can be taken as further evidence for spin-charge separation.

We now turn to the interesting case in which the superconducting susceptibility is enhanced due to the opening of a spin gap, $\Delta_{s}$. At temperatures large compared to $\Delta_{s}$, the spin gap can be ignored, and the spectral function is well approximated by that of the Tomonaga-Luttinger liquid. However, even below the spin gap scale, many of the characteristics of the TomonagaLuttinger spectral function are retained. Spin-charge separation still holds in the spin gapped Luther-Emery liquids and there are no stable "electronlike" excitations. The charge excitations are still the gapless charge density waves of the Tomonaga-Luttinger liquid but the spin excitations now consist of massive spin solitons with dispersion $E_{s}(k)=\sqrt{v_{s}^{2} k^{2}+\Delta_{s}^{2}}$. As a result 


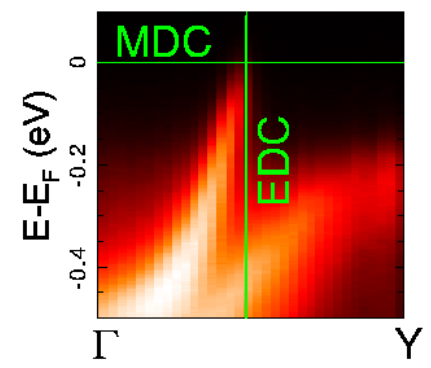

$$
\mathrm{Li}_{0.9} \mathrm{Mo}_{6} \mathrm{O}_{17}
$$

$\mathrm{T}=250 \mathrm{~K}$
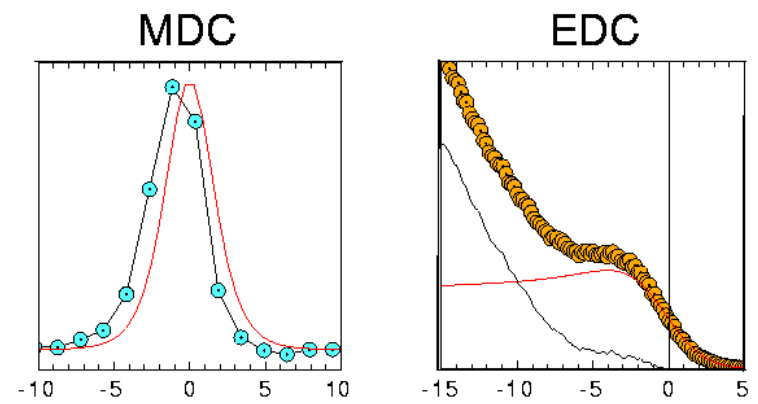

Fig. 17. ARPES intensity map for the purple bronze $\mathrm{Li}_{0.9} \mathrm{Mog}_{9} \mathrm{O}_{17}$. The lower left panel depicts the MDC at the Fermi energy together with a Tomonaga-Luttinger theoretical curve. The lower right panel contains the EDC at the Fermi wavevector. The red line corresponds to the Tomonaga-Luttinger result and the black curve is its deviation from the experimental data. (From Ref. 198.)

the spin piece of the spectral function is modified and from kinematics it follows that it consists of a coherent one spin soliton piece and an incoherent multisoliton part

$$
G_{s}(k, \omega)=Z_{s}(k) \delta\left[\omega+E_{s}(k)\right]+G_{s}^{(m u l t i)}(k, \omega),
$$

where the multisoliton piece is proportional (at $T=0)$ to $\Theta\left[-\omega-3 E_{s}(k / 3)\right]$. (For $K_{s}<1 / 2$ formation of spin soliton-antisoliton bound states, "breathers", may shift the threshold energy for multisoliton excitations somewhat). The form of $Z_{s}(k)$ has been calculated explicitly [199], but a simple scaling argument gleans the essential physics [149]. It follows from the fact that the Luther-Emery liquid is asymptotically free that at high energies and short distances compared to the spin gap, the physics looks the same as in the gapless state. Therefore the dependence of the correlation functions on high energy physics, such as the short distance cutoff $a$, cannot change with the opening of the gap. Since in the gapless system $G_{s}$ is proportional to $a^{2 \gamma_{s}-1 / 2}$, 
it is a matter of dimensional analysis to see that

$$
Z_{s}(k)=\left(\xi_{s} / a\right)^{\frac{1}{2}-2 \gamma_{s}} f_{s}\left(k \xi_{s}\right),
$$

where $f_{s}$ is a scaling function and $\xi_{s}=v_{s} / \Delta_{s}$ is the spin correlation length.

Despite the appearance of a coherent piece in the spin sector, the spectral function (Eq. 37) still exhibits an overall incoherent response owing to the convolution with the incoherent charge part. The result is grossly similar to the gapless case, aside from the fact that the Fermi edge (the tip of the triangular support of $A^{<}$in Fig. 15d) is pushed back from the Fermi energy by the magnitude of the spin gap (thus rounding the tip of the triangle). If, as suggested in Section 3, the Luther-Emery liquid is the paradigmatic example of a pseudogap state, clearly the above spectral function gives us an impression of what to expect the signature of the pseudogap to be in the one electron properties.

\subsection{Dimensional crossover in a quasi-1D superconductor}

Continuous global symmetries cannot be spontaneously broken in one dimension, even at $T=0$. Since the one dimensional Hamiltonian (Eq. (8)) is invariant under translations and spin $S U(2)$ and charge $U(1)$ transformations, no CDW, SDW, or superconducting long range order can exist in its ground state. Therefore, in a quasi-one dimensional system made out of an array of coupled 1DEG's, a transition into an ordered state necessarily signifies a dimensional crossover at which, owing to relevant interchain couplings, phases of individual chains lock together [23, 149]. The ultimate low temperature fate of the system is fixed by the identity of the first phase to do so. This, in turn, is determined by the relative strength of the various couplings and the nature of the low energy correlations in the 1DEG.

In the spin gapped phase, which we consider in the rest of this section, both the CDW and the superconducting susceptibilities are enhanced. To begin with, we will analyze the simplest model of a quasi-one dimensional superconductor. We defer until the following section any serious discussion of the competition between CDW and superconducting order. We will also defer until then any discussion of the richer possibilities which arise when the quasi-one dimensional physics arises from a self-organized structure, i.e. stripes, with their own additional degrees of freedom.

Interchain coupling and the onset of order The simplest and most widely studied model of a quasi-one dimensional spin gapped fluid is

$$
\begin{aligned}
\mathcal{H}= & \sum_{j} \mathcal{H}_{j}+\mathcal{J} \sum_{<i, j>}\left[O_{S S}^{\dagger}(i, x) O_{S S}(j, x)+\text { H.C. }\right] \\
& +\mathcal{V} \sum_{<i, j>}\left[O_{C D W}^{\dagger}(i, x) O_{C D W}(j, x)+\text { H.C. }\right]
\end{aligned}
$$

The Luther-Emery liquid is a pseudogap state. 
where $\mathcal{H}_{j}$ describes the Luther-Emery liquid on chain, pairs of nearest neighbor chains are denoted $\langle i, j\rangle$, and $O_{\alpha}(j, x)$ is the appropriate order parameter field on chain $j$. The bosonized form of these operators is given in Eqs. (24) and (25), above. It is assumed that the interchain couplings, $\mathcal{J}$ and $\mathcal{V}$, are small compared to all intrachain energies. There are two more or less complementary ways of approaching this problem:

1) The first is to perform a perturbative renormalization group (RG) analysis about the decoupled fixed point, i.e. compute the beta function perturbatively in powers of the interchain couplings. To lowest order, the beta function is simply determined by the scaling dimension, $D_{\alpha}$, of each operator - if $D_{\alpha}<2$, it means that $O_{\alpha}$ is perturbatively relevant, and otherwise it is irrelevant. It turns out that the CDW and SC orders are dual to each other, so that

$$
D_{S S}=1 / K_{c} \quad, \quad D_{C D W}=K_{c} .
$$

This has the implication that one, or the other, or both of the interchain couplings is always relevant. From this, we conclude with a high level of confidence that at low temperature, even if the interchain couplings are arbitrarily weak, the system eventually undergoes a phase transition to a higher dimensional ordered state. An estimate of $T_{c}$ can be derived from these equations in the standard way, by identifying the transition temperature with the scale at which an initially weak interchain coupling grows to be of order 1 . In this way, for $D_{S S}<2$, one obtains an estimate of the superconducting transition temperature

$$
T_{c} \sim E_{F}\left[\mathcal{J} / E_{F}\right]^{1 /\left(2-D_{S S}\right)}=\mathcal{J}\left[\mathcal{J} / E_{F}\right]^{\left(D_{S S}-1\right) /\left(2-D_{S S}\right)},
$$

and similarly for the CDW ordering temperature. Note that as $D_{S S} \rightarrow 2^{-}$, $T_{c} \rightarrow 0$, and that $T_{c} \gg \mathcal{J}$ for $D_{S S}<1$. Clearly, the power law dependence of $T_{c}$ on coupling constant offers the promise of a high $T_{c}$ when compared with the exponential dependence in BCS theory.

2) The other way is to use interchain mean field theory [200]. Here, one treats the one dimensional fluctuations exactly, but the interchain couplings in mean field theory. For instance, in the case of interchain SS ordering, one considers each chain in the presence of an external field

$$
\mathcal{H}^{e f f}=\mathcal{H}_{j}+\left[\Delta_{S S}^{*} O_{S S}(j, x)+\text { H.C. }\right],
$$

where $\Delta_{S S}$ is determined self-consistently:

$$
\Delta_{S S}=z \mathcal{J}\left\langle O_{S S}(j, x)\right\rangle,
$$

where $z$ is the number of nearest neighbor chains and the expectation value is taken with respect to the effective Hamiltonian. This mean field theory is exact 149,201 in the limit of large $z$, and is expected to be reliable so long as 
the interchain coupling is weak. It can be shown to give exact results in the limit of extreme anisotropy for the Ising model, even in two dimensions (where $z=2$ ) 200. More generally, it is a well controlled approximation at least for temperatures $T \gg \mathcal{J}$ (which includes temperatures in the neighborhood of $T_{c}$ as long as $D_{S S}<1$ ). This approach gives an estimate of $T_{c}$ which is related to the susceptibility of the single chain,

$$
1=z \mathcal{J} \chi_{S S}\left(T_{c}\right)
$$

which, from the expression in Eq. 32, can be seen to produce qualitatively the same estimate for $T_{c}$ as the perturbative RG treatment. The advantage of the mean field treatment is not only that it gives an explicit, and very physical expression for $T_{c}$, but that it permits us to compute explicitly the effect of ordering on various response functions, including the one particle spectral function. The case of CDW ordering is a straightforward extension.

Emergence of the quasiparticle in the ordered state The excitation spectrum changes dramatically below $T_{c}$ when the interchain "Josephson" coupling $\mathcal{J}$ triggers long range order 149. The fractionalized excitations of the Tomonaga-Luttinger and the Luther-Emery liquids are replaced by new excitations with familiar "BCS" quantum numbers. Formally, superconducting order leads to a confinement phenomenon. While the spin gap in the Luther-Emery state already implies suppressed fluctuations of $\phi_{s}$ on each chain, and correspondingly a finite amplitude $\cos \left(\sqrt{2 \pi} \phi_{s}\right)$ of the superconducting order parameter, it is the interchain Josephson coupling that tends to lock its phase $\theta_{c}$ from one chain to the next.

Operating with the hole operator, Eq. (9), on the ground state at the position of the $j$ th chain creates a pair of kinks (solitons) of magnitude $\sqrt{\pi / 2}$ in both the charge and spin fields $\theta_{c}$ and $\phi_{s}$ of this chain. As a result the phase of the order parameter changes by $\pi$ upon passing either the spin or the charge soliton. This introduces a negative Josephson coupling between the affected chain and its neighbors along the entire distance between the charge and spin solitons. The energy penalty due to this frustration grows linearly with the separation between the solitons and causes a bound pair to form. In fact, all solitonic excitations are confined into pairs, including charge-charge and spin-spin pairs. The bound state between the charge and the spin pieces restores the electron, or more precisely the Bogoliubov quasiparticle, as an elementary excitation, causing a coherent (delta function) peak to appear in the single particle spectral function.

An explicit expression for the spectral function in the superconducting state can be obtained in the context of the effective Hamiltonian in Eq. (47):

$$
A^{<}(k, \omega)=Z(k) \delta[\omega-E(k)]+A^{(\text {incoherent })}(k, \omega),
$$

where $E(k)=\sqrt{v_{s}^{2} k^{2}+\Delta_{0}^{2}}$. Here $\Delta_{0}=\Delta_{s}+\Delta_{c} / 2$ is the creation energy of the bound state where $\Delta_{c} \propto \Delta_{S S}$ is the mean field gap $\left(\Delta_{c} \ll \Delta_{s}\right)$ that

Superconducting order binds fractionalized excitations into "ordinary" quasiparticles. 
opens in the charge sector below $T_{c}$ 149. The multiparticle incoherent piece has a threshold slightly above the single hole threshold at $\omega=E(k)+2 \Delta_{c}$. The shape of $A^{<}(k, \omega)$ at $T=0$ is presented schematically in Fig. 18.

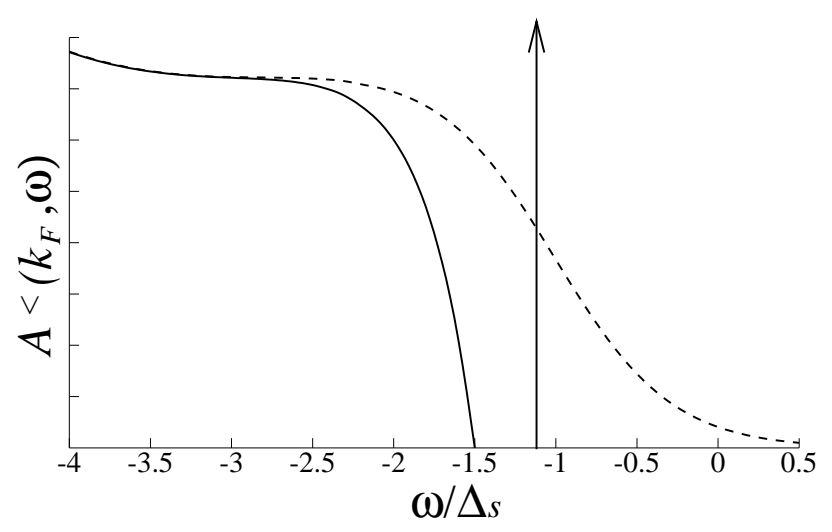

Fig. 18. The temperature evolution of the spectral function. The dashed line depicts $A^{<}$at intermediate temperatures below the spin gap $\Delta_{s}$ but above $T_{c}$. The solid line represents the spectral function at zero temperature. A coherent delta function peak onsets near $T_{c}$ at energy $\Delta_{0}=\Delta_{s}+\Delta_{c}(0) / 2$. The multiparticle piece starts at a threshold $2 \Delta_{c}(0)$ away from the coherent peak.

Once again, we may employ the asymptotic freedom of the system to construct a scaling argument. In this case, high energy physics dependent upon either the cutoff or the spin gap (which is by assumption much larger than $T_{c}$ ) cannot change upon entering the superconducting state. Comparing the form of the spectral response in the normal spin gapped state with that of the superconductor reveals the weight of the coherent peak

$$
Z(k)=Z_{s}(0)\left(\xi_{c} / a\right)^{-\frac{1}{2}-2 \gamma_{c}} f\left(k \xi_{c}\right),
$$

where $f$ is a scaling function and $\xi_{c}=v_{c} / \Delta_{c}$ is the charge correlation length. Physically, the dependence of the weight on $\Delta_{c}$, which also equals the (local) superfluid density 149, reflects the fact that the superfluid stiffness between chains controls the strength of the bound state forming the quasiparticle.

Since the superfluid density is a rapid function of temperature upon entering the superconducting state, the weight of the coherent peak will also rapidly increase as the temperature is lowered. Because the Josephson coupling is weak, the energy of the bound state is largely set by the spin gap, so that the energy of the coherent peak will not be a strong function of temperature in the neighborhood of $T_{c}$. Likewise, since the gap is not changing rapidly, the scattering rate and therefore the lifetime of the new quasiparticle will not have strong temperature dependence either. All of the above signa- 
tures have been observed in ARPES measurements of the coherent peak in $\mathrm{Bi}_{2} \mathrm{Sr}_{2} \mathrm{CaCu}_{2} \mathrm{O}_{8+\delta}$ [89, 91, 202, 203] and $\mathrm{YBa}_{2} \mathrm{Cu}_{3} \mathrm{O}_{7-\delta}$ [12].

The behavior we have just described is in sharp contrast to that of a conventional superconductor, where the gap opens precisely at $T_{c}$. Since in that case the gap is a rapid function of temperature, so is the energy of the conventional quasiparticle peak. Moreover, scattering processes are rapidly gapped out upon entering the BCS superconducting state, so that the quasiparticle often sharpens substantially as the temperature is lowered below $T_{c}$. Most importantly, in the conventional case, quasiparticles exist above the transition temperature, so the intensity ( $Z$ factor) of the peak does not change much upon entering the superconducting state. By contrast, in a quasi-one dimensional superconductor, there are no quasiparticle excitations in the normal state. The existence of the quasiparticle is due to the dimensional crossover to the three dimensional state, and is an entirely collective effect!

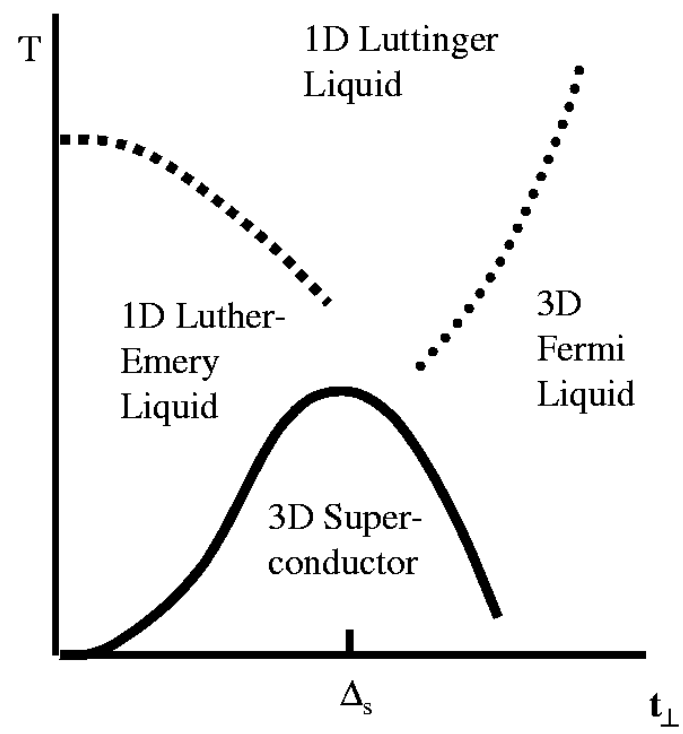

Fig. 19. Two Routes to Dimensional Crossover. In an array of multicomponent 1DEG's, for temperatures large compared to the transverse single particle tunnelling, $t_{\perp}$, the system behaves as a collection of independent (1D) Luttinger Liquids. For weak $t_{\perp}$, the dimensional crossover may proceed as described in Section 5.3, with a crossover first to a (1D) Luther-Emery Liquid, and a lower temperature dimensional crossover to a (3D) superconductor. For large $t_{\perp}$, there may be a dimensional crossover into a (3D) Fermi liquid, before the system becomes a (3D) superconductor.

The temperature evolution of the spectral function is in marked contrast with that in a BCS superconductor 


\subsection{Alternative routes to dimensional crossover}

Until now, we have assumed that the spin gap is large compared to the interchain couplings, and this assumption leads inevitably to the existence of a quasi-1D pseudogap regime above $T_{c}$ and a dimensional crossover associated with the phase ordering at $T_{c}$. Since under some circumstances, the spin gap in $1 \mathrm{D}$ can be zero or exponentially small compared to $E_{F}$, it is possible for a system to be quasi-1D, in the sense that the interchain couplings are small compared to the intrachain interactions, and yet have the dimensional crossover occur above any putative spin gap scale. In this case, most likely the dimensional crossover is triggered by the relevance of the interchain, single particle hopping operator - since any spin gap is negligible, the previous argument for its irrelevance is invalidated. What this means is that there is a dimensional crossover, $T_{3 D}^{*}$, at which the system transforms from a Luttinger liquid at high temperatures to a Fermi liquid at lower temperatures. (See Fig. 12.) If there are residual effective attractive interactions, the system will ultimately become a superconductor at still lower temperatures. However, in this case, the transition will be more or less of the BCS type - a Fermi

The case where dimensional crossover to a Fermi liquid occurs well above $T_{c}$ may serve as a model for the overdoped cuprates. surface instability (albeit on a highly anisotropic Fermi surface) with well defined quasiparticles existing both above and below $T_{c}$.

The crossover from a Luttinger liquid to a Fermi liquid is not as well characterized, theoretically, as the crossover to a superconductor. The reason is that no simple form of interchain mean field theory can be employed to study it. Various energy scales associated with the crossover can be readily obtained from a scaling analysis. A recent interesting advance [201, 204, 205] has been made on this problem using "dynamical mean field theory," again based on the idea of using $1 / z$ (where $z$ is the number of neighboring chains) as a small parameter, which gives some justification for a widely used RPAlike approximation for the spectral function 185. However, there are still serious shortcomings with this approximation [201, 206]. Clearly more interesting work remains to be done to sort out the physics in this limit, which may be a caricature of the physics of the overdoped cuprates. More complicated routes to dimensional crossover can also be studied [132], relevant to systems with more than one flavor of chain. For instance, it has recently been found that it is possible for a two component quasi-1D system to produce a superconducting state which supports gapless "nodal quasiparticles," even in the limit of extreme anisotropy 132.

\section{Quasi-1D Physics in a Dynamical Stripe Array}

Competition between $C D W$ and $S S$ is key in quasi-1D systems.
As mentioned before, in the simplest microscopic realizations of the 1DEG with repulsive interactions, $0<K_{c}<1$ and hence the CDW susceptibility is the most divergent as $T \rightarrow 0$ (See Eq. (32).) This seemingly implies that the typical fate of a quasi-one dimensional system with a spin gap is to wind up a CDW insulator in which CDW modulations on neighboring chains phase 
lock to each other. And, indeed, many quasi-one dimensional metals in nature suffer precisely this fate - the competition between CDW and SS order is a real feature of quasi-1D systems. Of course, as shown in Fig. 14, above, the $K_{c}$ inequality need not be satisfied in more complicated realizations of the 1DEG.

What we will examine in this section is another way in which the balance between CDW and SS ordering can be affected. [52, 207, 208] Specifically, we will show below that transverse fluctuations of the backbone on which the quasi-1D system lives significantly enhance the tendency to SS while suppressing CDW ordering. Such fluctuations are unimportant in conventional quasi-one dimensional solids, where the constituent molecules, upon which the electrons move, have a large mass and a rigid structure. But when the 1DEG's live along highly quantum electronic textures, or "stripes," transverse stripe fluctuations are probably always large.

\subsection{Ordering in the presence of quasi-static stripe fluctuations}

Consider a two dimensional array of stripes that run along the $x$ direction, and imagine that there is a 1DEG which lives on each stripe. To begin with, we will consider the case in which the stripe fluctuations are sufficiently slow that they can be treated as static - in other words, we consider an array of imperfectly ordered stripes, over whose meanderings we will eventually take an equilibrium (annealed) average. We will use a coordinate system in which points on the stripes are labeled by the coordinate $x$, the stripe number $j$, and in which transverse displacements of the stripe in the $y$ direction are labeled by $h_{j}(x)$. We therefore ignore the possibility of overhangs which is a safe assumption in the ordered state.

We now consider the effect that stripe geometry fluctuations have on the inter-stripe couplings. Because the CDW order (and any other $2 k_{F}$ or $4 k_{F}$ orders) occurs at a large wave vector, the geometric fluctuations profoundly affect its phase:

$$
O_{C D W}(j, x)=\frac{e^{-2 i k_{F} L_{j}(x)}}{\pi a} \cos \left[\sqrt{2 \pi} \phi_{s}(j, x)\right] e^{-i \sqrt{2 \pi} \phi_{c}(j, x)},
$$

where

$$
L_{j}(x)=\int_{0}^{x} d x^{\prime} \sqrt{1+\left(\partial_{x^{\prime}} h_{j}\right)^{2}},
$$

is the arc length, i.e. the distance measured along stripe $j$ to point $x$. At the same time $O_{S S}$ is unchanged, as are other $k=0$ orders. This results in a fundamental difference in the way CDW and Josephson inter-stripe couplings evolve with growing stripe fluctuations.

The CDW and Josephson couplings between neighboring stripes are of the form

$$
H_{\mathcal{V}}=\frac{1}{(2 \pi a)^{2}} \sum_{j} \int d x \mathcal{V}\left(\Delta_{j} h\right) \cos \left[\sqrt{2 \pi} \phi_{s}(j, x)\right] \cos \left[\sqrt{2 \pi} \phi_{s}(j+1, x)\right]
$$




$$
\begin{aligned}
& \times \cos \left[\sqrt{2 \pi} \Delta_{j} \phi_{c}+2 k_{f} \Delta_{j} L\right] \\
H_{\mathcal{J}}=-\frac{1}{(2 \pi a)^{2}} \sum_{j} & \int d x \mathcal{J}\left(\Delta_{j} h\right) \cos \left[\sqrt{2 \pi} \phi_{s}(j, x)\right] \cos \left[\sqrt{2 \pi} \phi_{s}(j+1, x)\right] \\
& \times \cos \left[\sqrt{2 \pi} \Delta_{j} \theta_{c}\right]
\end{aligned}
$$

where $\Delta_{j} h \equiv h(j+1, x)-h(j, x)$ etc. The coupling constants $\mathcal{V}\left(\Delta_{j} h\right)$ and $\mathcal{J}\left(\Delta_{j} h\right)$, depend on the local spacing between adjacent stripes, since they are more strongly coupled when they are close together than when they are far apart. This is particularly important for the Josephson coupling which depends on the pair tunnelling amplitude and therefore roughly exponentially on the local spacing between the stripes

$$
\mathcal{J}\left(\Delta_{j} h\right) \approx \mathcal{J}_{0} e^{-\alpha \Delta_{j} h} .
$$

By integrating out the stripe fluctuations $h$ one obtains the effective Hamiltonian of an equivalent rigid system of stripes. To first order in $\mathcal{V}$ the CDW coupling is similar to Eq. (54) but with $\Delta_{j} L$ set equal to 0 in the last term and $\mathcal{V}\left(\Delta_{j} h\right)$ replaced by

$$
\left\langle\mathcal{V}\left(\Delta_{j} h\right)\right\rangle \exp \left[-2 k_{F}^{2}\left\langle\left(\Delta_{j} L\right)^{2}\right\rangle\right],
$$

Stripe fluctuations dephase order...

... but they enhance SS order. where \langle\rangle signifies averaging over transverse stripe fluctuations. Since $\Delta_{j} L=$ $L_{j+1}(x)-L_{j}(x)$ is a sum of contributions with random signs, which are more or less independently distributed along the distance $|x|$, we expect it to grow roughly as in a random walk, i.e. $\left\langle\left(\Delta_{j} L\right)^{2}\right\rangle \sim D|x|$, where $D$ is a constant. Indeed one can show that at finite temperature $\left\langle\left(\Delta_{j} L\right)^{2}\right\rangle \sim T|x|$ while at $T=0\left\langle\left(\Delta_{j} L\right)^{2}\right\rangle \sim \hbar \bar{\omega} \log |x|$, where $\hbar \bar{\omega}$ is a suitable measure of the transverse stripe zero point energy. As a result of this dephasing effect, coupling between CDW's vanishes rapidly except in a narrow region near the ends of the stripes and hence can be ignored in the thermodynamic limit. In short, transverse stripe fluctuations cause destructive interference of $k \neq 0$ order on neighboring chains, strongly suppressing those orders.

The effects of stripe fluctuations on the Josephson coupling can be analyzed in the same way. To first order in the inter-stripe coupling, $\mathcal{J}\left(\Delta_{j} h\right)$ is simply replaced by its average value, $\overline{\mathcal{J}} \equiv<\mathcal{J}\left(\Delta_{j} h\right)>$. In other words, once quasi-static stripe fluctuations are integrated out, the result is once again the Hamiltonian we studied in Eq. (44), above, but with $\mathcal{V}=0$ and $\mathcal{J}=\overline{\mathcal{J}}$. Moreover, due to the exponential dependence of $\mathcal{J}\left(\Delta_{j} h\right)$ on $\left(\Delta_{j} h\right)$, it is clear that $\overline{\mathcal{J}}>\mathcal{J}(0)$, i.e. transverse stripe fluctuations strongly enhance the Josephson coupling between stripes. (There is a similar enhancement of the CDW coupling but it is overwhelmed by the dephasing effect.) Physically, this enhancement reflects the fact that the mean value of $\mathcal{J}$ is dominated by regions where neighboring stripes come close together. In the case of small amplitude fluctuations, this enhancemnt can be viewed as an inverse Debye-Waller factor,

$$
\langle\mathcal{J}\rangle \approx \mathcal{J}_{0} e^{\frac{\alpha^{2}}{2}\left\langle\left(\Delta_{j} h\right)^{2}\right\rangle}
$$


Where the transverse stripe fluctuations are comparable in magnitude to the inter-stripe spacing, the mean Josephson coupling is geometrically determined by the mean density of points at which neighboring stripes actually "bump" (i.e. separated by one lattice constant $a$ ). In this limit, treating the stripe fluctuations as a random walk yields the estimate

$$
\mathcal{J} \sim\left(\frac{a}{R}\right)^{2} \mathcal{J}_{0}
$$

where $R$ is the mean distance between stripes.

\subsection{The general smectic fixed point}

The quasi-static limit discussed above is presumably inadequate at low enough temperatures, where the quantum dynamics of stripe fluctuations must always be relevant. The complete problem, in which both the stripe dynamics and the dynamics of the 1DEG's are treated on an equal footing remains unsolved. However, since in a crystalline background, the stripe fluctuations are typically not gapless, we expect that at low enough temperatures, the stripe fluctuations can be treated as fast, and be integrated out to produce new effective interactions. So long as the stripes are reasonably smooth, these induced interactions will consist of long wavelength (around $k=0$ ) density-density and current-current interactions between the neighboring Luttinger liquidsinteractions that we have ignored until now. These interactions should undoubtedly be present in the bare model, as well, even in the absence of stripe fluctuations. They are marginal operators and should be included in the fixed point action 207, 209]. We are still interested in the spin gapped case so in the following analysis consider the charge sector only. Consequently we drop the subscript $c$ from the various quantities.

Using Eq. (11) and the bosonization formula for the current density along the chain, $-\sqrt{\frac{2}{\pi}} v K \partial_{x} \theta$, the phase-space Lagrangian density for $N$ coupled chains is

$$
\mathcal{L}=\sum_{j} \partial_{x} \theta_{j} \partial_{t} \phi_{j}-\frac{1}{2} \sum_{j, j^{\prime}=1}^{N}\left[\partial_{x} \phi_{j} \tilde{W}_{0}\left(j-j^{\prime}\right) \partial_{x} \phi_{j^{\prime}}+\partial_{x} \theta_{j} \tilde{W}_{1}\left(j-j^{\prime}\right) \partial_{x} \theta_{j^{\prime}}\right] .
$$

The diagonal terms $\left(j=j^{\prime}\right)$ in Eq. (60) describe the decoupled system with $\tilde{W}_{0}(0)=v / K$ and $\tilde{W}_{1}(0)=v K$. The off diagonal terms preserve the smectic symmetry $\phi_{j}(x) \rightarrow \phi_{j}(x)+\alpha_{j}$ and $\theta_{j}(x) \rightarrow \theta_{j}(x)+\beta_{j}$ (where $\alpha_{j}$ and $\beta_{j}$ are constant on each stripe) of the decoupled Luttinger fluids. Whenever this symmetry is unbroken, the $2 k_{F}$ charge density profiles and the superconducting order parameters on each stripe can slide relative to each other without an energy cost. This Hamiltonian thus describes a general "smectic metal phase." It is smectic in the sense that it can flow and has no resistance to shear, but it has a broken translational symmetry in the direction transverse "electron smectic". 
to the stripes - broken by the stripe array itself. Similar "sliding" phases of coupled classical two dimensional $X Y$ models have also been discussed [210].

The Lagrangian density in Eq. (60) can be simplified by integrating out the dual fields, and expressing the result in terms of the Fourier transform of $\phi_{a}$ with respect to the chain index, $\phi_{a}=\frac{1}{\sqrt{N}} \sum_{k_{\perp}} e^{i k_{\perp} a} \phi\left(k_{\perp}\right)$ :

$$
\mathcal{L}=\sum_{k_{\perp}} \frac{1}{2} \kappa\left(k_{\perp}\right)\left[\frac{1}{v\left(k_{\perp}\right)}\left|\partial_{t} \phi\left(k_{\perp}\right)\right|^{2}-v\left(k_{\perp}\right)\left|\partial_{x} \phi\left(k_{\perp}\right)\right|^{2}\right]
$$

where $\tilde{W}(a)=\frac{1}{N} \sum_{k_{\perp}} e^{i k_{\perp} a} W\left(k_{\perp}\right)$ so that the smectic fixed point is characterized by the $k_{\perp}$ dependent velocities and inverse Luttinger parameters

$$
\begin{aligned}
v\left(k_{\perp}\right) & =\sqrt{W_{0}\left(k_{\perp}\right) W_{1}\left(k_{\perp}\right)}, \\
\kappa\left(k_{\perp}\right) & =\sqrt{W_{0}\left(k_{\perp}\right) / W_{1}\left(k_{\perp}\right)} .
\end{aligned}
$$

Alternatively, in terms of the dual fields,

$$
\mathcal{L}=\sum_{k_{\perp}} \frac{1}{2 \kappa\left(k_{\perp}\right)}\left[\frac{1}{v\left(k_{\perp}\right)}\left|\partial_{t} \theta\left(k_{\perp}\right)\right|^{2}-v\left(k_{\perp}\right)\left|\partial_{x} \theta\left(k_{\perp}\right)\right|^{2}\right]
$$

In the presence of a spin gap, single electron tunnelling is irrelevant, and the only potentially relevant interactions involving pairs of stripes are singlet tunnelling and the coupling between the CDW order parameters, i.e., Eqs. (55, 54) with the cosine terms involving the spin fields replaced by their vacuum expectation values and with $\Delta_{j} L$ and $\Delta_{j} h$ set equal to 0 . The scaling dimensions of these perturbations can be readily evaluated [207, 209]:

$$
\begin{aligned}
D_{S C} & =\int_{-\pi}^{\pi} \frac{d k_{\perp}}{2 \pi} \kappa\left(k_{\perp}\right)\left(1-\cos k_{\perp}\right)=\kappa_{0}-\frac{\kappa_{1}}{2} \\
D_{C D W} & =\int_{-\pi}^{\pi} \frac{d k_{\perp}}{2 \pi} \frac{1}{\kappa\left(k_{\perp}\right)}\left(1-\cos k_{\perp}\right)=\frac{2}{\kappa_{0}-\kappa_{1}+\sqrt{\kappa_{0}^{2}-\kappa_{1}^{2}}} .
\end{aligned}
$$

Long wavelength couplings suppress $C D W$ even more.
To be explicit, in the above, we have (for purposes of illustration) evaluated the integrals for the simple model in which $\kappa\left(k_{\perp}\right)=\kappa_{0}+\kappa_{1} \cos k_{\perp}$. Here $\kappa_{0}$ can be thought of as the intra-stripe inverse Luttinger parameter and $\kappa_{1}$ is a measure of the nearest neighbor inter-stripe coupling. For stability, $\kappa_{0}>\kappa_{1}$ is required. Comparing the scaling dimensions in Eqs. (65) and (66) one obtains the phase diagram which is presented in Fig. 20. The line $\overrightarrow{A B}$ is a line of first order transitions between the smectic superconductor and the electronic crystal. It terminates at a bicritical point from which two continuous transition lines emanate. They separate the smectic superconductor and the crystal from a strong coupling regime where both Josephson tunnelling and CDW coupling are irrelevant at low energies. In this regime the smectic metal is stable. 


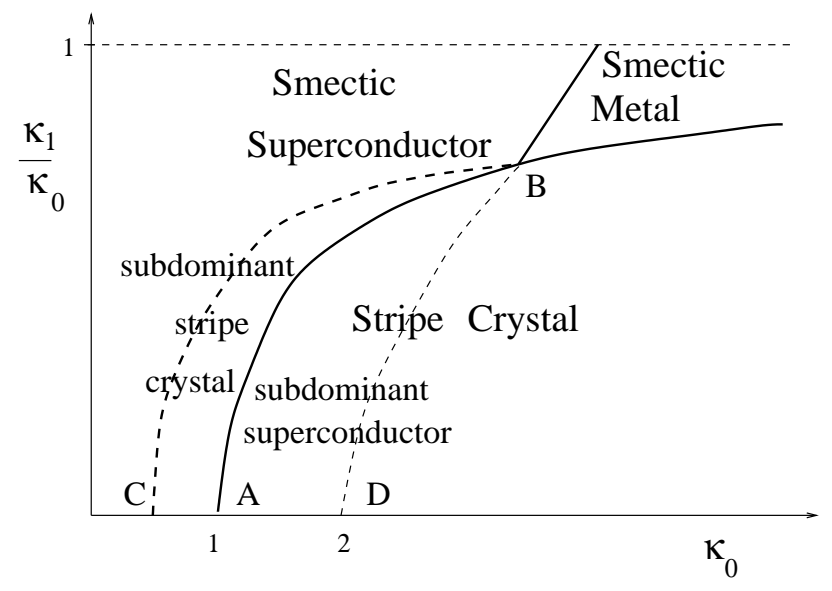

Fig. 20. Phase diagram of a spin gapped stripe array with model interactions as discussed in the text.

An important lesson from this model is that inter-stripe long wavelength interactions rapidly increase the scaling dimension of the inter-stripe CDW coupling while the scaling dimension of the Josephson coupling is less strongly affected (in this model it is actually reduced). Indeed one can see from Fig. 20 that there is a region of $\kappa_{0} \geq 1$ and large enough $\kappa_{1}$ where the global order is superconducting although in the absence of inter-stripe interactions $\left(\kappa_{1} \sim 0\right)$ the superconducting fluctuations are sub-dominant.

Extensions of this model to a three dimensional array of chains 211 and the inclusion of a magnetic field 212 have been considered as well. In particular, it is found that the magnetic field supresses the region of superconducting order in the phase diagram in Fig. (20), thus expanding the regime in which the smectic metal is stable. Similar considerations lead one naturally to consider other states obtained when the stripe fluctuations become still more violent. Assuming that the long range stripe order is destroyed by such fluctuations, while the short distance physics remains that of quasi-1DEG's living along the locally defined stripes, one is led to investigate the physics of electron nematic and stripe liquid phases. We shall return to this point in the final section.

\section{Electron Fractionalization in $D>1$ as a Mechanism of High Temperature Superconductivity}

We briefly discuss here a remarkable set of ideas for a novel mechanism of high temperature superconductivity based on higher dimensional generalizations of the 1D notion of spin-charge separation. Boasting a high pairing scale as well as crisp experimental predictions, these theories have many attractive 
features. They also bear a strong family resemblance to the "spin gap proximity effect mechanism," which we develop in some detail in Section 10.4. These appealing ideas, while valid, require the proximity of a spin liquid phase which in turn appears to be a fragile state of matter; for this reason, and others which will be made clear below, it is our opinion that these ideas are probably not applicable to the cuprate superconductors. The discussion in this section is therefore somewhat disconnected from the development in the rest of the paper. We merely sketch the central ideas, without providing any derivations. There are a number of recent papers dealing with this subject to which the interested reader can refer; see Refs. $77,80,183,213,214$.

\subsection{RVB and spin-charge separation in two dimensions}

Immediately following the discovery of high temperature superconductivity [2], Anderson proposed [5] that the key to the problem lay in the occurrence of a never before documented state of matter (in $D>1$ ), a spin liquid or "resonating valence bond" (RVB) state, related to a state he originally proposed 215] for quantum antiferromagnets on a triangular (or similarly frustrating) lattice. In this context [80], a spin liquid is defined to be an insulating state with an odd number of electrons per unit cell (and a charge gap) which breaks neither spin rotational nor translational symmetry. Building on this proposal, Kivelson, Rokhsar, and Sethna [69] showed that a consequence of the existence of such a spin liquid state is that there exist quasiparticles with reversed charge spin relations, just like the solitons in the 1DEG discussed in Section 5 , above. Specifically, there exist charge 0 spin $1 / 2$ "spinons" and charge e spin 0 "holons." Indeed, these quasiparticles were recognized as having a topological character [69,216] analogous to that of the Laughlin quasiparticles in the quantum Hall effect.

There was a debate at the time concerning the proper exchange statistics, with proposals presented identifying the holon as a boson [68, 69], a fermion [217], and a semion [218]. It is now clear that all sides of this debate were correct, in the sense that there is no universal answer to the question. The statistics of the fractionalized quasiparticles is dynamically determined, and is sensitive to a form of "topological order" [59, 78, 213, 217, 219] which differentiates various spin liquids. There are even transitions between states in which the holon has different statistics [219, 220].

Two features of this proposal are particularly attractive:

1) It is possible to envisage a high pairing scale in the Mott insulating parent state, since the strong repulsive interactions between electrons, which result in the insulating behavior, are insensitive to any subtler correlations between electrons. Thus, the " $\mu^{*}$ issue" does not arise: the spin liquid can be viewed as an insulating liquid of preformed cooper pairs [5, 69, 70], or 
equivalently a superconductor with zero superfluid density. If If this pairing scale is somehow preserved upon doping, then the transition temperature of the doped system is determined by superfluid stiffness and is not limited by a low pairing scale, as it would be in a BCS superconductor. Indeed, as in the case of the 1D Luther-Emery liquid discussed in Section 5 , pairing becomes primarily a property of the spin degrees of freedom, and involves little or no pairing of actual charge.

2) When the holons are bosonic, their density directly determines the superfluid density. Thus the superconducting $T_{c}$ can be crudely viewed as the bose condensation temperature of the holons. The result is that for small concentration of doped holes $x$ [5], the transition temperature is proportional to a positive power of $x$ (presumably [69] $T_{c} \sim x$ in 2D), in contrast to the exponential dependence on parameters in a BCS superconductor.

In short, many of the same features that would make a quasi-1D system attractive from the point of view of high temperature superconductivity (see Sections 5 and 10) would make a doped spin liquid even more attractive. However, there are both theoretical and phenomenological reasons for discounting this idea in the context of the cuprates.

\subsection{Is an insulating spin liquid ground state possible in $D>1$ ?}

The most basic theoretical issue concerning the applicability of the fractionalization idea is whether a spin liquid state occurs at all in $D>1$. The typical consequence of the Mott physics is an antiferromagnetically ordered ("spin crystalline") state, especially the Néel state, which indeed occurs at $x=0$ in the cuprates. Moreover, the most straightforward quantum disordering of an antiferromagnet will lead to a spin Peierls state, rather than a spin liquid, as was elegantly demonstrated by Haldane [221] and Read and Sachdev [71]. Indeed, despite many heroic efforts, the theoretical "proof of principle," i.e. a theoretically tractable microscopic model with plausible short range interactions which exhibits a spin liquid ground state phase, was difficult to achieve. A liquid is an intermediate phase, between solid and gas, and so cannot readily be understood in a strong or weak coupling limit 80 ].

Very recently, Moessner and Sondhi [79] have managed to demonstrate just this point of principle! They have considered a model [70] on a triangular lattice (thus returning very closely to the original proposal of Anderson) which is a bit of a caricature in the sense that the constituents are not single electrons, but rather valence bonds (hard core dimers), much in the spirit pioneered by Pauling. ${ }^{15}$ The model is sufficiently well motivated microscopically,

\footnotetext{
${ }^{14}$ An oxymoron since in this case $T_{\theta}=T_{c}=0$, but the intuitive notion is clear: we refer to a state which is derived from a superconductor by taking the limit of zero superfluid density while holding the pairing scale fixed.

${ }^{15}$ Indeed, it is tempting to interpret the dimer model as the strong coupling, high density limit of a fluid of Cooper pairs 70 .
}

Is this simply angels dancing on the head of a pin? 
Spin liquids are fragile.

The cuprates appear to be doped spin crystals, not doped spin liquids.

Where to look for spin liquids and the spin liquid character robust enough, that it is reasonable to declare the spin liquid a theoretical possibility. The spin liquid state of Moessner and Sondhi does not break any obvious symmetry. ${ }^{16}$

That said, the difficulty in finding such a spin liquid ground state in model calculations is still a telling point. A time reversal invariant insulating state cannot be adiabatically connected to a problem of noninteracting quasiparticles with an effective band structure ${ }^{17}$-band insulators always have an even number of electrons per unit cell. Thus, an insulating spin liquid is actually quite an exotic state of matter. Presumably, it only occurs when all more obvious types of ordered states are frustrated, i.e. those which break spin rotational symmetry, translational symmetry, or both. The best indications at present are that this occurs in an exceedingly small corner of model space, and that consequently spin liquids are likely to be rather delicate phenomena, if they occur at all in nature. This, in our opinion, is the basic theoretical reason for discarding this appealing idea in the cuprates, where high temperature superconductivity is an amazingly robust phenomenon.

One could still imagine that the insulating state is magnetically ordered, as indeed it is in the cuprates, but that upon doping, once the magnetic order is suppressed, the system looks more like a doped spin liquid than a doped antiferromagnet. In this context, there are a number of phenomenological points about the cuprates that strongly discourage this viewpoint. In the first place, the undoped system is not only an ordered antiferromagnet, it is a nearly classical one: its ground state and elementary excitation spectrum 222 225 are quantitatively understood using lowest order spin wave theory. This state is as far from a spin liquid as can be imagined! Moreover, even in the doped system, spin glass and other types of magnetic order are seen to persist up to (and even into) the superconducting state, often with frozen moments with magnitude comparable to the ordered moments in the undoped system 225 228]. These and other indications show that the doped system "remembers" that it is a doped antiferromagnet, rather a doped spin liquid.

Regardless of applicability to the cuprates, it would be worthwhile to search for materials that do exhibit spin liquid states, and even more so to look for superconductivity when they are doped. Numerical studies [229 232] indicate that good candidates for this are electrons on a triangular lattice with substantial longer range ring exchange interactions, such as may occur in a 2D Wigner crystal near to its quantum melting point [233], and the Kagomé lattice. It is also possible, as discussed in Section 11, to look for superconductivity in systems that exhibit some form of spin-charge separation at intermediate length scales. (See also Ref. 14.)

\footnotetext{
16 This work was, to some extent, anticipated in studies of large $N$ generalizations of the Heisenberg antiferromagnet. 71

${ }^{17}$ In a time reversal symmetry broken state, the band structure need not exhibit the Kramer's degeneracy, so that a weak coupling state with an odd number of electrons per unit cell is possible.
} 


\subsection{Topological order and electron fractionalization}

Finally, we address the problem of classifying phases in which true electron fractionalization occurs, e.g. in which spinons are deconfined. It is now clear from the work of Wen [78 and Senthil and Fisher [59] that the best macroscopic characterization of fractionalized phases in two or more dimensions is topological, since they frequently possess no local order parameter. Specifically, a fractionalized phase exhibits certain predictable ground state degeneracies on various closed surfaces - degeneracies which Senthil and Fisher have given a physical interpretation in terms of "vison expulsion." Unlike the degeneracies associated with conventional broken symmetries, these degeneracies are not lifted by small external fields which break either translational or spin rotational symmetry. It has even been shown [59,219, 234. (as funny as this may sound) that topological order is amenable to experimental detection. Once topological classification is accepted, the one to one relation between spin liquids and electron fractionalization, implied in our previous discussion, is eliminated. Indeed, it is possible to imagine [59,76] ordered (broken symmetry) states, proximate to a spin liquid phase, which will preserve the ground state degeneracies of the nearby spin liquid, and hence will exhibit spin-charge separation.

\section{Superconductors with Small Superfluid Density}

A hallmark of BCS theory is that pairing precipitates order. But it is possible for the two phenomena to happen separately: pairing can occur at a higher temperature than superconductivity. In this case, there is an intermediate temperature range described by electron pairs which have not condensed. In the order parameter language, this corresponds to a well developed amplitude of the order parameter, but with a phase which varies throughout the sample. Superconductivity then occurs with the onset of long range phase coherence. (This is how ordering occurs in a quasi-1D superconductor, as discussed in Section 5 , above.) Such superconductors, while they may have a large pairing scale, have a small stiffness to phase fluctuations, or equivalently a small superfluid density.

\subsection{What ground state properties predict $T_{c}$ ?}

When the normal state is understood, it is reasonable to describe superconductivity as an instability of the normal state as temperature is lowered, which BCS theory does quite successfully in simple metals. Another approach, useful especially when the normal state is not well understood, is to consider which thermal fluctuations degrade the superconducting order as the temperature is raised. Put another way, we address the question, "What measurable ground state $(T=0)$ properties permit us to predict $T_{c}$ ?" 
Pairing is one energy scale...

...the superfluid phase stiffness sets another.

Of this there is no possible doubt whatever.
Two classes of thermal excitations are responsible for disordering the ground state of a superconductor: amplitude fluctuations of the complex order parameter (associated with pair breaking), and fluctuations of the phase (associated with pair currents).

The strength of the pairing at $T=0$ is quantifiable as a typical gap value, $\Delta_{0}$, where

$$
T_{p} \equiv \Delta_{0} / 2,
$$

is the characteristic temperature at which the pairs fall apart. In a BCS superconductor, it is possible to estimate that $T_{c} \approx T_{p}$. (The factor $1 / 2 \mathrm{in}$ this definition approximates the weak coupling BCS expression, $T_{c}=\Delta_{0} / 1.78$.) Certainly, more generally, $T_{p}$ marks a loose upper bound to $T_{c}$, since if there is no pairing, there is probably no superconductivity.

We can construct another ground state energy scale as follows: Divide the sample into blocks of linear dimension, $L$, and ask how much energy it costs to flip the sign of the superconducting order parameter at the center of one such region. So long as $L$ is larger than the coherence length, $\xi_{0}$, the cheapest way to do this is by winding the phase of the order parameter, so the energy is determined by the superfluid phase stiffness

$$
T_{\theta}=\frac{1}{2} A \gamma L^{d-2}
$$

where $d$ is the number of spatial dimensions, $A$ is a geometry dependent dimensionless number of order 1 and the "helicity modulus", $\gamma$, is traditionally expressed in terms of the ratio of the superfluid density, $n_{s}$, to the pair effective mass, $m^{*}$ :

$$
\gamma \equiv \frac{\hbar^{2} n_{s}}{m^{*}}
$$

(We will discuss the quantitative aspects of this relation in Subsection 8.3.) Note that for $d=2$, this energy is independent of $L$, while for $d=3$ it is minimized for the smallest allowable value of $L \sim \xi_{0}$. Clearly, when the temperature is comparable to $T_{\theta}$, thermal agitation will produce random phase changes from block to block, and hence destroy any long range order. Again, a rough upper bound to $T_{c}$ is obtained in this way.

In short, it is possible to conclude on very general grounds that

$$
T_{c} \leq \min \left[T_{p}, T_{\theta}\right]
$$

When $T_{p} \ll T_{\theta}$, phase fluctuations can be completely neglected except in the immediate neighborhood of $T_{c}$ - this is the case in BCS superconductors. If $T_{p} \gg T_{\theta}$, quasiparticle excitations, i.e. the broken Cooper pairs, play no significant thermodynamic role up to $T_{c}$. In this case a considerable amount of local pairing, and consequently a pseudogap, must persist to temperatures well above $T_{c}$. When both $T_{p}$ and $T_{\theta}$ are comparable to $T_{c}$, as is the case in most optimally doped high temperature superconductors, neither class of thermal excitation can be safely neglected. 
Table 1.

\begin{tabular}{|c|c|c|c|c|c|c|}
\hline Material & $L[\AA]$ & $\lambda_{L}[\AA]$ & $T_{p}[\mathrm{~K}]$ & $T_{c}[\mathrm{~K}]$ & $T_{\theta}[\mathrm{K}]$ & Ref. \\
\hline$\overline{\mathrm{Pb}}$ & $\overline{830}$ & $\overline{390}$ & $\overline{7.9}$ & $\overline{7.2}$ & $6 \times 10^{5}$ & 235,236 \\
\hline $\mathrm{Nb}_{3} \mathrm{Sn}$ & 60 & 640 & 18.7 & 17.8 & $2 \times 10^{4}$ & 237 \\
\hline $\mathrm{UBe}_{13}$ & 140 & 10,000 & 0.8 & 0.9 & $10^{2}$ & 238240 \\
\hline $\mathrm{Ba}_{0.6} \mathrm{~K}_{0.4} \mathrm{BiO}_{3}$ & 40 & 3000 & 17.4 & 26 & $5 \times 10^{2}$ & 241,242 \\
\hline $\mathrm{K}_{3} \mathrm{C}_{60}$ & 30 & 4800 & 26 & 20 & $10^{2}$ & 243245 \\
\hline $\mathrm{MgB}_{2}$ & 50 & 1400 & 15 & 39 & $1.4 \times 10^{3}$ & \begin{tabular}{|l|l|}
246 & 248 \\
\end{tabular} \\
\hline$\overline{\overline{\mathrm{ET}}}$ & 15.2 & 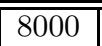 & 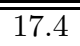 & $\overline{\overline{10.4}}$ & $\overline{15}$ & 249.250 \\
\hline $\mathrm{NCCO}$ & 6.0 & 1600 & 10 & $21-24$ & 130 & $?, ?$ \\
\hline$\overline{\mathrm{PCCO}}$ & 6.2 & 2800 & 23 & 23 & 86 & 251253 \\
\hline Tl-2201 (op) & 11.6 & & 122 & 91 & & 254 \\
\hline Tl-2201 (od) & 11.6 & 2000 & & 80 & 160 & 250,255 \\
\hline Tl-2201 (od) & 11.6 & 2200 & & 48 & 130 & 250,255 \\
\hline Tl-2201 (od) & 11.6 & & 26 & 25 & & 256 \\
\hline Tl-2201 (od) & 11.6 & 4000 & & 13 & 40 & 250,255 \\
\hline Bi-2212 (ud) $x=.11$ & 7.5 & & 275 & 83 & & 97,257 \\
\hline $\mathrm{Bi}-2212$ (op) & 7.5 & & 220 & 95 & & 257 \\
\hline Bi-2212 (op) & 7.5 & 2700 & & $90-93$ & 60 & \begin{tabular}{|l|l|}
251 & 258 \\
\end{tabular} \\
\hline $\mathrm{Bi}-2212$ (op) & 7.5 & 1800 & & 84 & 130 & 259,260 \\
\hline $\mathrm{Bi}-2212(\mathrm{od}) \mathrm{x}=.19$ & 7.5 & & 143 & 82 & & 257 \\
\hline $\mathrm{Bi}-2212(\mathrm{od}) \mathrm{x}=.225$ & 7.5 & & 104 & 62 & & 257 \\
\hline $\mathrm{Y}-123(\mathrm{ud}) \mathrm{x}=.075$ & 5.9 & 2800 & & 38 & 42 & 261 \\
\hline $\mathrm{Y}-123(\mathrm{ud}) \mathrm{x}=.1$ & 5.9 & 1900 & & 64 & 90 & 261 \\
\hline $\mathrm{Y}-123(\mathrm{op}) \mathrm{x}=.16$ & 5.9 & 1500 & & 85.5 & 140 & 261,262 \\
\hline Y-123 (op) & 5.9 & & 116 & $91-92$ & & 99 \\
\hline $\mathrm{Y}-123(\mathrm{od}) \mathrm{x}=.19$ & 5.9 & 1300 & & 79 & 180 & 261 \\
\hline $\mathrm{Y}-123$ (od) $\mathrm{x}=.23$ & 5.9 & 1500 & & 55 & 140 & 261 \\
\hline $\mathrm{Y}-248$ & 6.8 & 1600 & & 82 & 150 & 263 \\
\hline Hg-1201 (op) & 9.5 & 1700 & 192 & $95-97$ & 180 & \begin{tabular}{|l|l|}
262 & 264 \\
\end{tabular} \\
\hline $\mathrm{Hg}-1212$ (op) & 6.4 & 1700 & 290 & 108 & 130 & 264,265 \\
\hline Hg-1223 (op) & 5.3 & 1500 & 435 & $132-135$ & 130 & $262,264,265$ \\
\hline Hg-1223 (op) & 7.9 & 1500 & & 135 & 190 & 262,265 \\
\hline LSCO (ud) $x=.1$ & 6.6 & 2800 & 75 & 30 & 47 & \begin{tabular}{|l|l|}
266 & 268 \\
\end{tabular} \\
\hline LSCO (op) $x=.15$ & 6.6 & 2600 & 58 & 38 & 54 & 266. 267 \\
\hline LSCO (od) $\mathrm{x}=.20$ & 6.6 & 1950 & & 34 & 96 & 267 \\
\hline LSCO (od) $\mathrm{x}=.22$ & 6.6 & 1900 & & 27 & 100 & 267 \\
\hline LSCO (od) $\mathrm{x}=.24$ & 6.6 & 1900 & & 20 & 100 & 267 \\
\hline
\end{tabular}


Caption for Table 1: Zero temperature properties of the superconducting state as predictors of $T_{c}$. Here, $T_{p}$ is computed from Eq. (67) using values of $\Delta_{0}$ obtained from either tunnelling or ARPES, except for overdoped Tl-2201, for which we have used Raman data. In computing $T_{\theta}$ from Eq. (68) for nearly isotropic materials (those above the double line), we have taken $d=3, A=2.2, L=\sqrt{\pi} \xi_{0}$, and $n_{s} / 2 m^{*}=(8 \pi)^{-1}(c / e)^{2} \lambda_{L}^{-2}$ where $\lambda_{L}$ and $\xi_{0}$ are the zero temperature London penetration depth and coherence length, respectively. For layered materials, we have taken $d=2, A=0.9$, and the areal superfluid density $n_{s} / 2 m^{*}=(8 \pi)^{-1}(c / e)^{2} L \lambda_{L}^{-2}$ where $L$ is now the mean spacing between layers and $\lambda_{L}$ is the in-plane London penetration depth. The precise numerical values of $A$ and the factor of $\sqrt{\pi}$ should not be taken seriously - they depend on microscopic details, which can vary from material to material as discussed in Section 8.3. Penetration depth measurements on Y-123 refer to polycrystalline $\mathrm{Y}_{0.8} \mathrm{Ca}_{0.2} \mathrm{Ba}_{2} \mathrm{Cu}_{3} \mathrm{O}_{7-\delta}$, and report $\lambda_{a b}$. The two entries for $\mathrm{Hg}-1223$ assume that the superfluid density resides in all three planes $(\mathrm{L}=5.3 \AA)$, or the outer two planes only $(\mathrm{L}=7.9 \AA)$. In the case of the high temperature superconductors, the notations 'ud', 'op', and 'od' refer to under, optimally, and overdoped materials, respectively.

In Table 1, following Ref. 269, we tabulate $T_{\theta}, T_{p}$, and $T_{c}$ for various superconducting materials. Clearly, in bulk $\mathrm{Pb}$, phase fluctuations are not terribly important, while in the cuprate superconductors (and the ET superconductors), phase fluctuations are an order 1 effect. Of this there is no possible doubt! Looking more closely at the table, one sees that the ratio of $T_{\theta} / T_{c}$ is generally smaller for the underdoped materials, and larger for overdoped, which implies that phase fluctuations are progressively less dominant with increasing doping. The ratio of $T_{p} / T_{c}$ varies in the opposite manner with doping.

The obvious implication of the trends exhibited in Table 1 is that optimal doping marks a gradual crossover from an underdoped regime, where $T_{c}$ is predominantly a phase ordering transition, to an overdoped regime in which it is predominantly a pairing transition. This also implies that both pairing and phase fluctuation physics play a nonnegligible role, except in the regimes of extreme underdoping or overdoping where $T_{c} \rightarrow 0$.

\subsection{An illustrative example: granular superconductors}

We now turn to a beautiful set of experiments carried out by Merchant et al. 270 on granular $\mathrm{Pb}$ films with a thin coating of $\mathrm{Ag}$. This is a system in which the microscopic physics is well understood. The $T_{c}$ of bulk $\mathrm{Pb}$ is $7.2 \mathrm{~K}$ while Ag remains normal down to the lowest accessible temperatures, so that $T_{\theta}$ can be varied with respect to $T_{p}$ by changing the thickness of Ag. In this way, the system can be tuned from an "underdoped" regime, where $T_{c}$ is a phase ordering transition and pairing persists to much higher temperatures, to an "overdoped" regime, where the transition is very BCS-like.

Figure 21 shows the log of the resistance vs. temperature for a sequence of films (a-j) obtained by adding successive layers of $\mathrm{Ag}$ to a granular $\mathrm{Pb}$ 


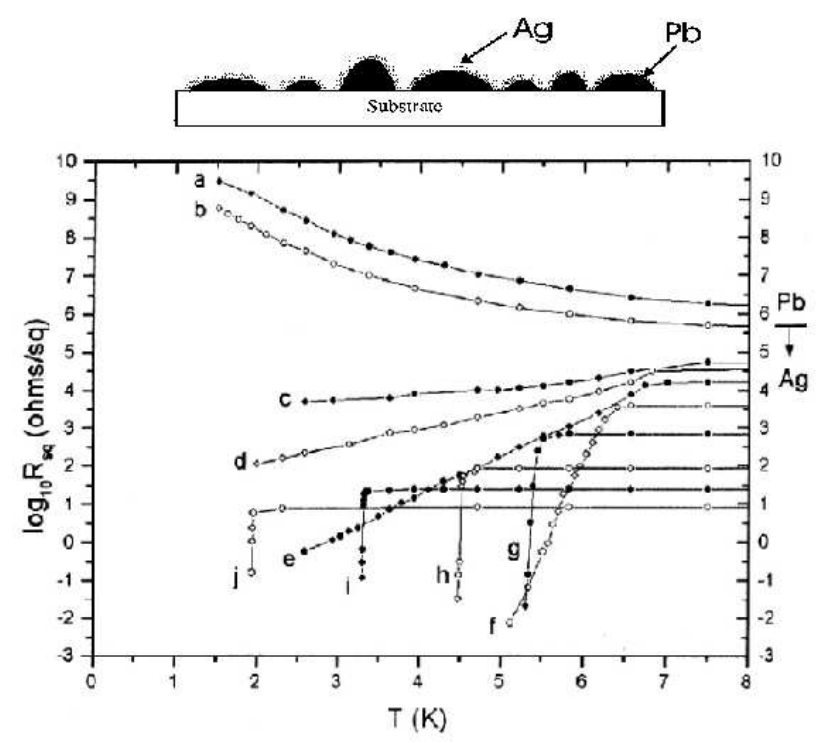

Fig. 21. The logarithm of the resistance vs. temperature for a sequence of films, starting with a granular $\mathrm{Pb}$ film (a) to which is added successively larger coverage of Ag. From Fig. 5 of Merchant et al. [270.

substrate. Films a and $\mathrm{b}$ are seen to be globally insulating, despite being locally superconducting below $7.2 \mathrm{~K}$. Films g-j are clearly superconductors. Films c-f are anomalous metals of some still not understood variety. It is important to note that Fig. 21 is plotted on a log-linear scale, so that although it is unclear whether films c-f will ever become truly superconducting, films e and $\mathrm{f}$, for example, have low temperature resistances which are 5 or 6 orders of magnitude lower than their normal state values, due to significant superconducting fluctuations; see Fig. 22.

Figure 23 shows I-V curves obtained from planar tunnelling in the direction perpendicular to the same set of films. As $d I / d V$ is proportional to the single particle density of states at energy $V$, this can be interpreted as the analogue of an ARPES or tunnelling experiment in the high temperature superconductors. Among other things, the gap seen in films a-d is roughly independent of $\mathrm{Ag}$ coverage, and looks precisely like the gap that is seen upon tunnelling into thick $\mathrm{Pb}$ films. In these films, the gap seen in tunnelling is clearly a superconducting pseudogap.

The analogy between the behavior of these films as a function of $\mathrm{Ag}$ coverage, and the cuprate high temperature superconductors as a function of hole concentration is immediately apparent: 


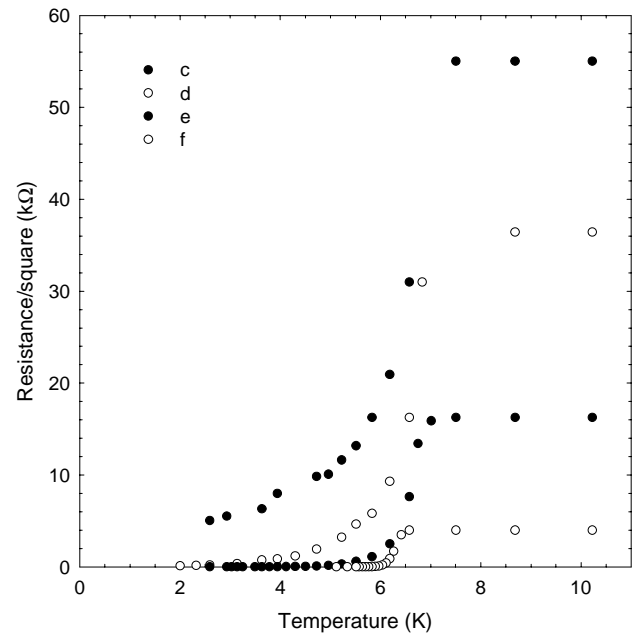

Fig. 22. The same data as in Fig. (21), but on a linear, as opposed to a logarithmic, scale of resistivity.

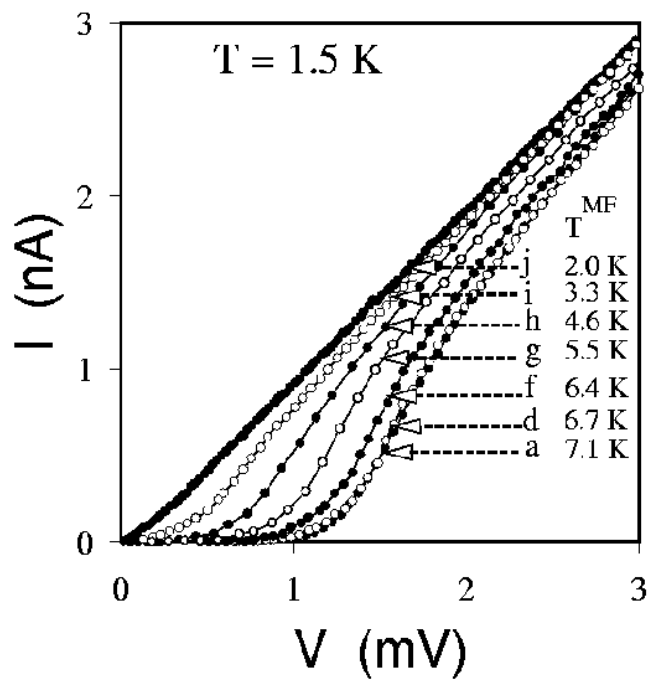

Fig. 23. I-V curves from planar tunnelling into the same sequence of films shown in Fig. (21). From Fig. 6 of Merchant et al 270.

With little or no Ag, the typical Josephson coupling, $J$, between far sepa" $T_{c}$ " increases with increasing Ag... rated grains of $\mathrm{Pb}$ is small; thermal phase fluctuations preclude any possibility of long range phase order for $T>J$. Clearly, increasing Ag coverage increases the coupling between grains, or more correctly, since the granular character 
of the films is gradually obscured with increasing Ag coverage, it increases the phase stiffness or superfluid density. This causes the phase ordering temperature to rise, much like the underdoped regime of the cuprates.

However, the pairing scale, or equivalently the mean field $T_{c}$, is a decreasing function of $\mathrm{Ag}$ coverage due to the proximity effect. Since the $\mathrm{Pb}$ grains are small compared to the bulk coherence length, $\xi_{0}$, the granularity of the films has little effect on the BCS gap equation. The pairing scale is equivalent to that of a homogeneous system with an effective pairing interaction,

$$
\lambda^{e f f}=\lambda_{P b} \times f_{P b}+0 \times f_{A g},
$$

where $f_{P b}$ and $f_{A g}$ are, respectively, the volume fraction of $\mathrm{Pb}$ and $\mathrm{Ag}$. Consequently, the pairing gap,

$$
\Delta_{0} \sim \exp \left[-1 /\left(\lambda^{e f f}-\mu^{*}\right)\right]
$$

is a decreasing function of Ag coverage. So long as $f_{A g} \ll 1$ (films a-f) this effect is rather slight, as can be seen directly from the figures, but then the gap value can be seen to plummet with increasing Ag coverage. In films g-j, this leads to a decrease of $T_{c}$, reminiscent of overdoped cuprates.

Of course, it is clear that there is more going on in the experiment than this simple theoretical discussion implies:

1) Disorder: The effects of disorder are neglected in this discussion. A priori these should be strong, especially at low Ag coverage.

2) Coulomb Blockade: As best one can tell from the existing data, films a-f are not superconductors with a reduced $T_{c}$ - in fact films a and b appear to be headed toward an insulating ground state, presumably due to quantum phase fluctuations induced by the charging energy of the grains. The energy to transfer a Cooper pair (charge 2e) between grains is

$$
V_{C}=4 \alpha e^{2} / L
$$

where $L$ is the grain size and $\alpha$ is a dimensionless constant which takes into account the grain shape and screening. When $V_{C}>J$, the number of pairs per grain becomes fixed at low temperature and the ground state is a type of paired Mott insulator. Since the number of pairs and the phase are quantum mechanically conjugate on each grain, when number fluctuations are suppressed by the charging energy, quantum phase fluctuations flourish, and prevent superconducting order. The screening of the Coulomb interaction can mitigate this effect. Screening clearly improves with increasing Ag coverage, so coverage dependent effects of quantum phase fluctuations contribute to the evolution observed in the experiments, as well.

3) Dissipation: There is even more to this story than the $\omega=0$ charging energies. In contrast with classical statistical mechanics, the dynamics and the thermodynamics are inexorably linked in quantum statistical mechanics, and finite frequency physics becomes important. This issue has been 
A mysterious ground state

The superfluid density sets the phase stiffness. addressed experimentally by Rimberg et al. 271] While there has been considerable progress in understanding the theory of quantum phase fluctuations (See, for example, Ref. 272 for a recent review), there are still many basic issues that are unresolved. For instance, films c-f show no sign of becoming truly superconducting or insulating as $T \rightarrow 0$ ! What is the nature of this intermediate state? This is a widely observed phenomenon in systems which are expected to be undergoing a superconductor to insulator transition 272, 273. The physics of this anomalous metallic state is not understood at all, even in systems, such as the present one, where the microscopic physics is believed to be understood. (See Section 8.4 for a taste of the theoretical subtleties involved.)

\subsection{Classical phase fluctuations}

We now undertake a critical analysis of thermal phase fluctuations. We will for now ignore the effects of thermal quasiparticle excitations, as well as the quantum dynamics which certainly dominate the phase mode physics at temperatures low compared to its effective Debye temperature. These important omissions will be addressed in Section 8.4.

Superconductors and classical XY models When $T_{\theta} \ll T_{p}$, the superconducting transition temperature $T_{c} \approx T_{\theta}$, and the transition can be well described by a phase only model. On general symmetry grounds, the free energy associated with time independent deformations of the phase must be of the form

$$
V_{\text {phase }}=(\gamma / 2) \int d \boldsymbol{r}(\nabla \theta)^{2},
$$

where the helicity modulus, $\gamma$, is given by the superfluid density, $n_{s}$, and the effective pair mass, $m^{*}$, according to Eq. (69). Since $\boldsymbol{v}_{s}=\frac{\hbar}{m^{*}} \nabla \theta$ is the superfluid velocity, $V_{\text {phase }}$ is easily seen to have an interpretation as the kinetic energy of the superfluid, $V_{\text {phase }}=\int d \boldsymbol{r} n_{s} m^{*} v_{s}^{2} / 2$, so that classical phase fluctuations correspond to thermally induced pair currents. Eqs. (74) and (69) establish the sense in which the superfluid density controls the stiffness to phase fluctuations.

Eq. (74) is the continuum form of the classical XY model. Both in a superconductor and in the XY model, $\theta$ is a periodic variable (defined modulo $2 \pi)$. Thus, we must handle the short distance physics with some care to permit the vortex excitations which are the expression of that periodicity. When this is done, typically by defining the model on a lattice, it captures the essential physics of the transition between a low temperature ordered and a high temperature disordered state.

To be concrete, let us consider an XY model on a $d$ dimensional hypercubic lattice

$$
H_{X Y}=-\sum_{<i, j>} \mathcal{V}\left(\theta_{i}-\theta_{j}\right)
$$


where $\langle i, j\rangle$ are nearest neighbor sites and $\mathcal{V}$ is an even, periodic function $\mathcal{V}(\theta)=\mathcal{V}(\theta+2 \pi)=\mathcal{V}(-\theta)$, with a maximum at $\theta=0$ such that the Hamiltonian is minimized by the uniform state. The lattice constant, $a$, in this model has a physical interpretation - it defines the size of the vortex core. To generalize this model to the case of an anisotropic (e.g. layered) superconductor, we let both the lattice constant, $a_{\mu}$, and the potential, $\mathcal{V}_{\mu}(\theta)$, depend on the direction, $\mu$.

At zero temperature, the helicity modulus can be simply computed:

$$
\gamma_{\mu}(T=0)=2\left[a_{\mu}^{2} / \nu\right] \mathcal{V}_{\mu}^{\prime \prime}(0)
$$

where $\nu=\left(\prod_{\nu} a_{\nu}\right)$ is the unit cell volume. Thus, the relation between $\gamma(0)$ and $T_{\theta}$, the ordering temperature of the model, depends both on the detailed form of $\mathcal{V}$ and on the lattice cutoff. In constructing Table 1 above, we have taken $\mathcal{V}=V \cos (\theta)$, and identified the area of the vortex core, $\pi \xi_{0}^{2}$, with the plaquette area, $a^{2}$ - this is the origin of the somewhat arbitrary $\sqrt{\pi}$ which appears in the three dimensional expression for $T_{\theta}$. Fortuitously, for layered materials, $\gamma_{x}=\gamma_{y} \equiv \gamma_{x y}$ depends only on the spacing between planes, $a_{z}$, and not on the in-plane lattice constant.

One can, in principle, handle the short distance physics in a more systematic way by solving the microscopic problem (probably numerically) on large systems (large compared to $\xi_{0}$ ), and then matching the results with the short distance behavior of the XY model. In this way, one could, in principle, derive explicit expressions for $\mathcal{V}$ and $a_{\mu}$ in terms of the microscopic properties of a given material. However, no one (to the best of our knowledge) has carried through such an analysis for any relevant microscopic model.

What we 274 have done, instead, is to keep at most the first 2 terms in a Fourier cosine series of Eq. (75). With the cuprates in mind, we have studied planar systems:

$$
\begin{aligned}
H= & -J_{\|} \sum_{<i j>_{\|}}\left\{\cos \left(\theta_{i j}\right)+\delta \cos \left(2 \theta_{i j}\right)\right\} \\
& -J_{\perp} \sum_{<i j>_{\perp}}\left\{\cos \left(\theta_{i j}\right)\right\},
\end{aligned}
$$

where $<i j>_{\|}$denotes nearest neighbors within a plane, and $<i j>_{\perp}$ denotes nearest neighbors between planes. It is assumed that $J_{\|}, J_{\perp}$, and $\delta$ are positive, since there is no reason to expect any frustration in the problem, 275 and that $\delta \leq 0.25$, since for $\delta>0.25$ there is a secondary minimum in the potential for $\theta_{i j}=\pi$, which is probably unphysical. Since dimensional analysis arguments of the sort made above are essentially independent of $\delta$, varying $\delta$ permits us to obtain some feeling for how quantitatively robust the results are with regard to "microscopic details."

Properties of classical XY models The XY model is one of the most studied models in physics 276. We 274 have recently carried out a series of 
quantitative analytic and numerical studies of XY models (using Eq. (77)). In particular, we have focused on the thermal evolution of the superfluid density and the relation between the superfluid density and the ordering temperature.

As long as $J_{\perp}$ is nonzero, this model is in the universality class of the $3 \mathrm{D}$ $\mathrm{XY}$ model, and near enough to $T_{c}, \gamma(T) \sim\left|T_{c}-T\right|^{\nu}$, where $\nu$ is the correlation length exponent of the $3 \mathrm{DXY}$ model, $\nu \approx .67$. For sufficient anisotropy, there may be a crossover from 2D critical behavior close (but not too close) to $T_{c}$, to $3 \mathrm{D}$ critical behavior very near $T_{c}$. In practice, this crossover is very hard to see due to the special character of the critical phenomena of the 2D XY model; even a very weak $J_{\perp}$ significantly increases the transition temperature.

To see this, consider the case in which $J_{\|} \gg J_{\perp}$; in this limit, one can study the physics of the system using an asymptotically exact interplane mean field theory [200]. We define the order parameter, $m(T) \equiv\left\langle\cos \left[\theta_{j}\right]\right\rangle$, and consider the behavior of a single decoupled planar $X Y$ model in the presence of an external field, $h(T)=2 J_{\perp} m(T)$ due to the mean field of the neighboring two planes. The self-consistency condition thus reads

$$
m(T)=m_{2 D}(T, h),
$$

where $m_{2 D}(T, h)$ is computed for the $2 \mathrm{D}$ model. A simple estimate for $T_{c}$ can be obtained by linearizing this equation:

$$
1=2 J_{\perp} \chi_{2 D}\left(T_{c}\right) .
$$

$2 D$ critical behavior may be hard to see.

The superfluid density is linear at low $T$.
Here the 2D susceptibility is

$$
\chi_{2 D} \sim T_{2 D}^{-1} \exp \left\{A_{\chi} \sqrt{T_{2 D} /\left(T_{c}-T_{2 D}\right)}\right\},
$$

where $T_{2 D}$ is the Kosterlitz-Thouless transition temperature and $A_{\chi}$ is a nonuniversal number of order 1 . A consequence of this is that even a very small interlayer coupling leads to a very large fractional increase in $T_{c}$

$$
T_{c}-T_{2 D} \sim T_{2 D} A_{\chi}^{2} / \log ^{2}\left[J_{\|} / J_{\perp}\right] .
$$

Only if $\left(T_{c}-T_{2 D}\right) / T_{2 D} \ll 1$ will there be clear $2 \mathrm{D}$ critical behavior observed in the thermodynamics.

To make contact with a range of experiments it is necessary that we focus attention not only on universal critical properties, but also on other properties which are at least relatively robust to changes in microscopic details. One such property is the width of the critical region, but we are not aware of any systematic studies of the factors that influence this. For the simple $(\delta=0)$ isotropic 3D XY model, the critical region certainly does not extend further than $10 \%$ away from $T_{c}$.

Another such property is the low temperature slope of superfluid density curves as a function of temperature. Using linear spin wave theory [277, 278], one can obtain a low temperature expansion of the in-plane helicity modulus, 


$$
\frac{\gamma_{\|}(T)}{a_{\perp}}=J_{\|}(1+4 \delta)-\frac{\alpha(1-16 \delta)}{4(1+\delta)} T+\mathcal{O}\left(T^{2}\right),
$$

where we have used $a_{x}=a_{y} \equiv a_{\perp}$ and $\gamma_{x}=\gamma_{y} \equiv \gamma_{\|}$for a planar system and $\alpha$ is a nonuniversal number which depends on $J_{\perp} / J_{\|}$. It is easy to show 274] that $\alpha=1$ in the two dimensional limit $\left(J_{\perp} / J_{\|}=0\right)$, and that $\alpha=2 / 3$ in the three dimensional limit $\left(J_{\perp}=J_{\|}(1+4 \delta)\right)$. The $T$-linear term is independent of $J_{\|}$, so that we expect the slope of scaled superfluid density curves, $\gamma_{\|}(T) / \gamma_{\|}(0)$ vs. $T / T_{c}$, to be much less sensitive to microscopic parameters (i.e. material dependent properties such as doping in the cuprates) than is $\gamma_{\|}(0)$. That this expectation is realized can be seen from our Monte Carlo simulation results presented in Fig. 24.

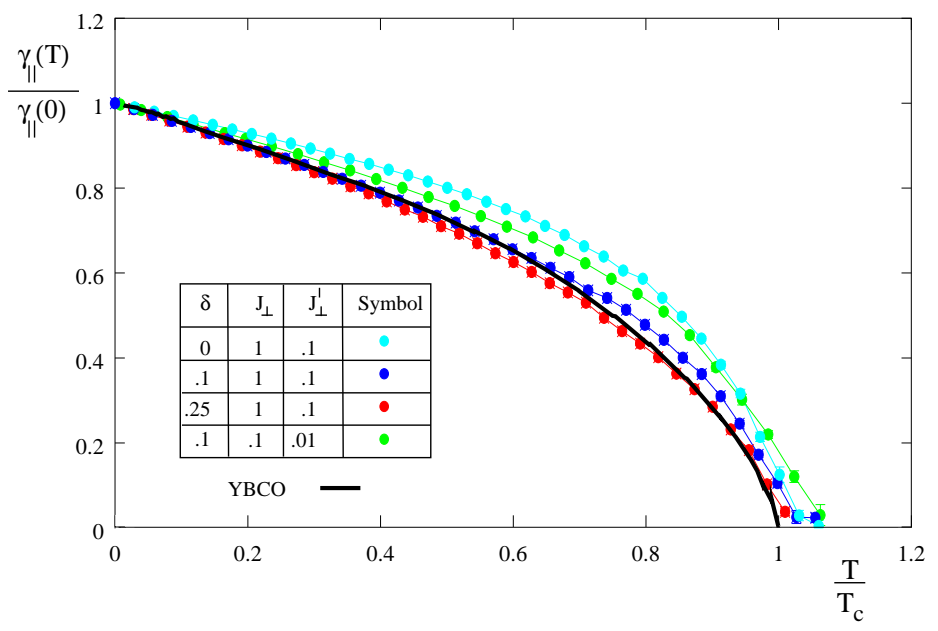

Fig. 24. Superfluid density vs. temperature, scaled by the zero temperature superfluid density and by $T_{c}$, respectively, from Ref. 274. Experimental data on $Y B C O$ is depicted by the black line, and is taken from Kamal et al. 279] (The data are essentially the same for a range of doping concentration.) Our Monte Carlo results for system size $16 \times 16 \times 16$ are the filled symbols. Calculations are for two planes per unit cell, with coupling $J_{\|}=1$ within each plane, and $J_{\perp}$ and $J_{\perp}^{\prime}$ between alternate planes. Monte Carlo points above $T_{c}$ are nonzero due to finite size effects. Except where explicitly shown, error bars are smaller than symbol size.

In addition, we find that there is a characteristic shape to the superfluid density vs. temperature curves in XY models. We have used Monte Carlo simulations to focus on two other dimensionless nonuniversal parameters: $A_{1}=T_{c} / \gamma_{\|}(0)$ and $A_{2}=T_{c} \gamma_{\|}^{\prime}(0) / \gamma_{\|}(0)$, where $\gamma_{\|}^{\prime}(0)=d \gamma_{\|}(0) / d T$. $A_{1}$ is a measure of how well the ground state property $\gamma_{\|}(0)$ (measurable through the superfluid density) predicts $T_{c}$, which is equivalent to $T_{\theta}$ in this model. 
The shape of $\gamma(T)$ is robust!

The order of limits matters.
$A_{2}$ can be expressed in the more intuitive form $A_{2}=T_{c} / T_{e x}$, where $T_{e x} \equiv$ $\gamma_{\|}(0) / \gamma_{\|}^{\prime}(0)$, is the estimate of $T_{c}$ one would obtain by extrapolating from the low temperature slope of $\gamma_{\|}(T)$ to the point at which the superfluid stiffness would vanish. Over orders of magnitude of couplings $\left(0 \leq J_{\perp} / J_{\|} \leq .1\right)$, and throughout the range $0 \leq \delta \leq .25, A_{1}$ and $A_{2}$ are remarkably robust: $A_{1} \sim .6-1.7$, and $A_{2} \sim .2-.5$.

\subsection{Quantum considerations}

In quantum systems, the dynamics affects the thermodynamics. However, the role of quantum effects on the phase dynamics is a large topic, and one in which many uncertainties remain. We will briefly discuss the simplest case here, mostly to illustrate the complexity of the problem.

Let us consider a simple two fluid model [3] in which a phase fluctuating superconductor is capacitively coupled to a normal fluid. The continuum limit of the effective action obtained upon integrating out the normal fluid can be derived from simple hydrodynamic considerations. From the Josephson relation, it follows that the electric field

$$
\boldsymbol{E}=-(\hbar / 2 e) \boldsymbol{\nabla} \dot{\theta}
$$

The Euclidean effective action is obtained by augmenting the classical action, Eq. (74), with the Maxwell term, and analytically continuing to imaginary time:

$$
S[\theta]=\int_{0}^{\beta} d \tau\left\{\int d \boldsymbol{r} \mathcal{L}_{\text {quantum }}+V_{\text {phase }}\right\}, \quad \mathcal{L}_{\text {quantum }}=\boldsymbol{E} \cdot \boldsymbol{D} / 8 \pi,
$$

where $\beta=1 / T, \boldsymbol{D}(\boldsymbol{k}, \omega)=\epsilon_{0}(\boldsymbol{k}, \omega) \boldsymbol{E}(\boldsymbol{k}, \omega)$, and $\epsilon_{0}$ is the normal fluid dielectric function (analytically continued to imaginary time). Again, this effective action must be cutoff at short distances in such a way as to preserve the periodicity of $\theta$ by allowing vortex excitations.

An analysis of the Maxwell term, $S_{\text {quantum, }}$, allows us to illustrate some of the complexity of this problem. At $\boldsymbol{k}=\mathbf{0}$ and small $\omega, \epsilon_{0} \approx 4 \pi \sigma_{0} / i \omega$, where $\sigma_{0}$ is the D.C. conductivity of the normal fluid. Thus, if we first consider the spatial continuum limit before going to low frequencies, $S_{\text {quantum }} \sim$ $\sum_{\omega_{n}} \int d \boldsymbol{r} \sigma_{0}\left|\omega_{n}\right||\boldsymbol{\nabla} \theta|^{2}$, where $\omega_{n}=2 \pi n T$ are the Matsubara frequencies. We recognize the resulting action as the continuum limit of an array of resistively shunted Josephson junctions 280,281 (RSJ). Here, the normal fluid plays the role of an "Ohmic heat bath."

On the other hand, if we first take $\omega=0$, and then $k$ small, $\epsilon \approx\left(k_{T F} / k\right)^{2}$ where $k_{T F}$ is the Thomas-Fermi screening length. In this limit, the Maxwell term has the form of a phase kinetic energy, $\mathcal{L}_{\text {quantum }} \sim\left(M_{\theta} / 2\right)|\dot{\theta}|^{2}$, with an effective mass, $M_{\theta} \propto\left[e^{2} / k_{T F}^{2}\right]^{-1}$ inversely proportional to an appropriately defined local charging energy. The resulting effective action is the continuum limit of the "lattice quantum rotor" (QR) model, also a widely studied 
problem [282]. The RSJ and QR models have quite different behavior at low temperatures. Without a rather complete understanding of the physics of the normal fluid, it is impossible in general to determine which, if either, of these limits captures the essential quantum physics.

There is nonetheless one important issue which can be addressed in a theoretically straightforward fashion: the temperature scale below which quantum effects dominate. The classical physics we studied in the previous section is readily obtained from the quantum model by suppressing all fluctuations with nonzero Matsubara frequency. We thus estimate a classical to quantum crossover temperature, $T_{c l}$, by comparing the classical $(\omega=0)$ and first finite frequency $\left(\omega=\omega_{1}=2 \pi T\right)$ contributions to $S[\theta]$. This leads to the implicit equation for $T_{c l}$ :

$$
T_{c l}=\sqrt{e^{2} n_{s} / \epsilon_{0} m^{*}},
$$

where $\epsilon_{0}$ is evaluated at temperature $T=T_{c l}$, frequency $\omega \sim 2 \pi T_{c l}$, and a typical momentum, $k \sim 1 / a$. So long as $T \gg T_{c l}$, the imaginary time independent (classical) field configurations dominate the thermodynamics. Clearly, depending on how good the screening is, $T_{c l}$ can be much smaller or much larger than $T_{c}$. If we approximate $\epsilon_{0}$ by its finite frequency, $k \rightarrow 0$ form, this estimate can be recast in an intuitively appealing form [274]:

$$
T_{c l} \sim\left(\frac{\sigma_{Q}}{\sigma_{0}}\right) T_{\theta}
$$

where $\sigma_{Q}=e^{2} /(h a)$ is the quantum of conductance in which the vortex core radius enters as the quantum of length.

Recent theoretical developments have uncovered yet more subtleties. Although the low energy physics involves only phase fluctuations, phase slips (short imaginary time events where the phase spontaneously "slips" by $2 \pi$ ) involve amplitude fluctuations. In the presence of an ohmic heat bath, there are subtle, long time consequences of these amplitude fluctuations 283285. Another interesting possibility is electron fractionalization. Under some circumstances, it has been proposed 59 that $h c / e$ vortices may be energetically preferred to the usual $h c / 2 e$ vortices, leading to a fractionalized state.

Combine this exciting but incomplete jumble of theoretical ideas with the remarkably simple but entirely unexplained behavior observed experimentally in granular superconducting films as they crossover from superconducting to insulating behavior, and one is forced to concede that the theory of quantum phase fluctuations is seriously incomplete.

\subsection{Applicability to the cuprates}

Both phase and pair breaking fluctuations are more prevalent at low $T$ in the cuprate superconductors than in conventional BCS superconductors. The low superfluid density provides only a weak stiffness to thermal phase fluctuations of the order parameter. In addition, the nodes in the gap mean that there
The classical to quantum crossover temperature is estimated.

This is an important unsolved problem! 
There is no signature of the transition in the single particle gap. are low energy quasiparticle excitations down to arbitrarily low temperature. However, it is important to remember that nodal quasiparticles occupy only a small fraction of the Brillouin zone so long as $\Delta_{o} \gg T$.

$T_{c}$ is unrelated to the gap in underdoped cuprates As mentioned in Section 3, in underdoped cuprates, many probes detect a pseudogap in the normal state, such as NMR, STM, junction tunnelling, and ARPES. Whereas BCS theory would predict $T_{c} \sim \Delta_{o} / 2$, where $\Delta_{o}$ is the superconducting gap maximum at zero temperature, the low temperature magnitude of the single particle gap as measured by ARPES or tunnelling experiments does not follow this relation, qualitatively or quantitatively. On the underdoped side, $T_{c}$ increases with increasing doping, whereas $\Delta_{o}$ moves in the opposite direction in all cases studied to date. Even at optimal doping, $T_{c}$ is always considerably smaller than the BCS value of $\Delta_{o} / 2$. In optimally doped BSCCO, for example, $T_{c} \sim \Delta_{o} / 5$, where $\Delta_{o}$ is the peak energy observed in low temperature tunnelling experiments. 150,286,287 (See also Table 1.)

The ARPES experiments provide $k$-space information demonstrating that the gap, above and below $T_{c}$, has an anisotropy consistent with a $d$-wave order parameter. Furthermore, $\Delta_{o}(T)$ is largely undiminished in going from $T=0$ to $T=T_{c}$ in underdoped samples, and the size and shape of the gap are basically unchanged through the transition. Add to this the contravariance of $T_{c}$ with the low temperature magnitude of the gap as the doping is changed, and it appears the gap and $T_{c}$ are simply independent energies [134,288]. The gap decreases with overdoping, which may be responsible for the depression of $T_{c}$ in that region, so that the transition may be more conventional on the overdoped side.

$T_{c}$ is set by the superfluid density in underdoped cuprates As emphasized above, the superfluid density in cuprates is orders of magnitude smaller than in conventional superconductors. [269] In addition, when the superfluid density is converted to an energy scale, it is comparable to $T_{c}$, whereas in conventional superconductors this phase stiffness energy scale is far above the transition temperature. In those conventional cases, BCS theory works quite well, but in the cuprates, the phase stiffness energy scale should also be considered.

This is further emphasized by the Uemura plot 107, which compares the transition temperature to the superfluid density. For underdoped systems, the relationship is linear within experimental errors. This is strong evidence that $T_{c}$ is determined by the superfluid density, and therefore set by phase ordering.

Experimental signatures of phase fluctuations In YBCO, $3 D X Y$ critical fluctuations have been observed in the superfluid density within $10 \%$ of 
$T_{c}$ [172, 289, implying that the temperature dependence of the superfluid density below and near $T_{c}$ is governed by phase fluctuations. It needs to be stressed that in conventional superconductors, such fluctuations that are seen are Gaussian in character - that is they involve fluctuations of both the amplitude and the phase of the order parameter 18 . The purely critical phase fluctuations observed in YBCO are entirely different. At low temperature (as low as $T=1 \mathrm{~K}$ [291]), the superfluid density is a linearly decreasing function of temperature [9]. While this linear behavior is generally believed to be the result of amplitude fluctuations of an order parameter with nodes, it is difficult [148, 151, 292, 293] from this perspective to understand why the slope is nearly independent of $x$ and of $\Delta_{0} / T_{c}$. This feature of the data is naturally explained if it is assumed that the linear temperature dependence, too, arises from classical phase fluctuations, but then it is hard to understand [274] why quantum effects would not quench these fluctuations at such low temperatures.

\section{Lessons From Weak Coupling}

\subsection{Perturbative RG approach in $D>1$}

In recent years, Fermi liquid theory, and with it the characterization of the BCS instability, has been recast in the language of a perturbative renormalization group (RG) treatment. We will adopt this approach as we reconsider the conventional BCS-Eliashberg theory of the phonon mediated mechanism of superconductivity in simple metals. In particular, we are interested in exploring the interplay between a short ranged instantaneous electron-electron repulsion of strength $\mu$ and a retarded attraction (which we can think of as being mediated by the exchange of phonons) of strength $\lambda$, which operates only below a frequency scale $\omega_{D}$. Although we will make use of a perturbative expression for the beta function which is valid only for $\mu$ and $\lambda$ small compared to 1 , the results are nonperturbative in the sense that we will recover the nonanalytic behavior of the pairing scale, $T_{p}$, expected from BCS mean field theory. The results are valid for any relative strength of $\mu / \lambda$ and, moreover, the corrections due to higher order terms in the beta function are generally smooth, and so are not expected to have large qualitative effects on the results so long as $\mu$ and $\lambda$ are not large compared to 1 .

All the results obtained in this section have been well understood by experts since the golden age of many-body theory, along with some of the most important higher order corrections which occur for $\lambda$ of order 1 (which will be entirely neglected here). Our principal purpose in including this section is to provide a simple derivation of these results in a language that may be more accessible to the modern reader. A most insightful exposition of this approach

18 An interesting way to identify separate Gaussian and phase fluctuation regimes in YBCO is presented in Ref. 290. See also Ref. 79 . 
is available in the articles by Polchinski, Ref. 39, and Shankar, Ref. 38, which can be consulted wherever the reader is curious about parts of the analysis we have skipped over. The one technical modification we adopt here is to employ an energy shell RG transformation, rather than the momentum shell approach adopted in Ref. 38; this method allows us to handle the retarded and instantaneous interactions on an equal footing. It can also be viewed as an extension of the analogous treatment of the $1 \mathrm{D}$ problem adopted in Ref. 294, as discussed in the next subsection.

We start by defining a scale invariant (fixed point) Euclidean action for a noninteracting Fermi gas

$$
\begin{aligned}
S_{f p}\left[\Psi_{\uparrow}, \Psi_{\downarrow}\right] & =(2 \pi)^{-(d+1)} k_{F}^{d-1} \sum_{\sigma} \int d \omega d \hat{k} d k \mathcal{L}_{0}\left[\Psi_{\sigma}\right], \\
\mathcal{L}_{0}\left[\Psi_{\sigma}\right] & =\bar{\Psi}_{\sigma}\left[i \omega+v_{F}(\hat{k}) k\right] \Psi_{\sigma},
\end{aligned}
$$

where $d \boldsymbol{k}=k_{F}^{d-1} d \hat{k} d k$, the unit vector $\hat{k}$ is the direction of $\boldsymbol{k}$ and $k$ is the displacement from the Fermi surface; we have assumed a simple spherical Fermi surface. The treatment that we present here breaks down when the Fermi surface is nested or contains Van Hove singularities. To regularize the theory, it is necessary to cut off the integrals; whereas Shankar confines $k$ to a narrow shell about the Fermi surface, $|k|<\Lambda \ll k_{F}$, we allow $k$ to vary from $-\infty$ to $+\infty$, but confine the $\omega$ integral to a narrow shell $|\omega|<\Omega \ll E_{F}$.

We now introduce electron-electron interactions. Naive power counting leads to the conclusion that the four fermion terms are marginal, and all higher order terms are irrelevant, so we take

$$
\begin{aligned}
S_{i n t}= & \sum_{\sigma, \sigma^{\prime}} \int \prod_{j=1}^{3} \frac{d \boldsymbol{k}_{j} d \omega_{j}}{(2 \pi)^{d+1}} \bar{\Psi}_{\sigma}\left(\boldsymbol{k}_{1}, \omega_{1}\right) \bar{\Psi}_{\sigma^{\prime}}\left(\boldsymbol{k}_{2}, \omega_{2}\right) \\
& \times\left[g\left(\boldsymbol{k}_{2}-\boldsymbol{k}_{3}\right)+\Theta\left(\omega_{D}-\left|\omega_{2}-\omega_{3}\right|\right) \tilde{g}\left(\boldsymbol{k}_{2}-\boldsymbol{k}_{3}\right)\right] \\
& \times \Psi_{\sigma^{\prime}}\left(\boldsymbol{k}_{3}, \omega_{3}\right) \Psi_{\sigma}\left(\boldsymbol{k}_{1}+\boldsymbol{k}_{2}-\boldsymbol{k}_{3}, \omega_{1}+\omega_{2}-\omega_{3}\right),
\end{aligned}
$$

where $\Theta$ is the Heavyside function, and $g$ and $\tilde{g}$ are, respectively, the instantaneous and retarded interactions. Signs are such that positive $g$ corresponds to repulsive interactions. The distinction between retarded and instantaneous interactions is important so long as $\Omega \gg \omega_{D}$. We have invoked spin rotation invariance in order to ignore the dependence of $g$ and $\tilde{g}$ on the spin indices.

It should be stressed, as already mentioned in Section 5 , that this should already be interpreted as an effective field theory, in which the microscopic properties that depend on the band structure away from the Fermi surface such as mixing with other bands, more complicated three and four-body interactions, etc. have already fed into the parameters that appear in the model. What we do now is to address the question of what further changes in the effective interactions are produced when we integrate out electronic 
modes in a narrow shell between $\Omega$ and $\Omega e^{-\ell},(\ell>0$ and small), and then rescale all frequencies according to

$$
\omega \rightarrow e^{\ell} \omega, \quad k \rightarrow e^{\ell} k \text { and } \Psi \rightarrow e^{-\left(3 / 2+\eta_{F}\right) \ell} \Psi,
$$

to restore the cutoff to its original form and where, as usual, $\eta_{F}$ is a critical exponent that is determined by the the properties of the interacting fixed point. We will carry this procedure out perturbatively in powers of $g$ and $\tilde{g}$ - to the one loop order we (and everyone else) analyzes, $\eta_{F}=0$.

To first order in perturbation theory, simple power counting insures that the entire effective action is invariant under the RG transformation, other than the parameter $\omega_{D}$ which changes according to

$$
d \omega_{D} / d \ell=\omega_{D} .
$$

a)

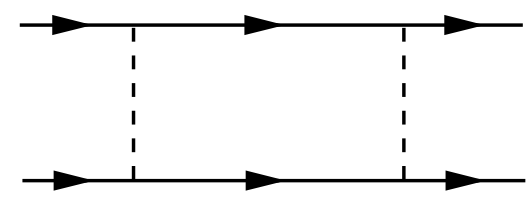

b)

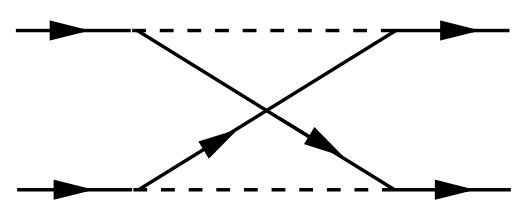

c)

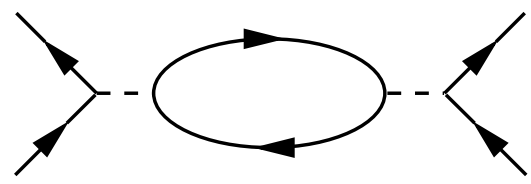

d)

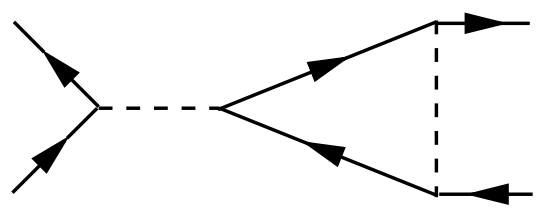

Fig. 25. The one loop diagrams that are invoked in the discussion of the renormalization of the effective interactions. a) and b) are referred to as the "Cooper channel" and c) and d) as "particle-hole channels". The loop is made out of electronic propagators with frequencies in the shell which is being integrated. The dashed lines represent interactions. 
To second (one loop) order, the forward scattering interactions are still unchanged; they produce the Fermi liquid parameters, and should actually be included as part of the fixed point action and treated non-peturbatively. This can be done straightforwardly, but for simplicity will be ignored here. The one loop diagrams which potentially produce contributions to the beta function are shown in Fig. 25. All internal legs of the diagrams refer to electron propagators at arbitrary momenta but with their frequencies constrained to lie in the shell which is being integrated out, $\Omega>|\omega| \geq \Omega e^{-\ell}$. The dashed lines represent interactions. All external legs are taken to lie on or near the Fermi surface. Clearly, the energy transfer along the interaction lines in the Cooper channel, Figs. 25a and 25b, is of order $\Omega$, and so for $\Omega \gg \omega_{D}, \tilde{g}$ does not contribute, while in Figs. 25c and 25/d there is zero frequency transfer along the interaction lines, and so $g$ and $\tilde{g}$ contribute equally.

Since $\Omega \ll E_{F}$, we can classify the magnitude of each diagram in powers of $\Omega$; any term of order $|\Omega|^{-1}$ makes a logarithmically divergent contribution to the effective interaction upon integration over frequency, while any terms that are proportional to $E_{F}^{-1}$ are much smaller and make only finite contributions which can be ignored for the present purposes. When the Cooper diagrams, shown in Figs. 25a and 25b, are evaluated for zero center of mass momentum, (i.e. if the momenta on the external legs are $\boldsymbol{k}_{F}$ and $-\boldsymbol{k}_{F}$ ), the bubble is easily seen to be proportional to $\Omega^{-1}$. However, if the center of mass momentum is nonzero (i.e. if the external momenta are $\boldsymbol{k}_{F}+\boldsymbol{q}$ and $-\boldsymbol{k}_{F}$ ), the same bubble is proportional to $1 / v_{F}|\boldsymbol{q}|$, and hence is negligible. The particle-hole diagrams in Figs. 25c and 25d are a bit more complicated. The bubble is zero for total momentum 0 , and proportional to $1 / v_{F} k_{F}$ for momentum transfer near $2 k_{F}$. Thus, in more than one dimension, the particle hole bubbles can be neglected entirely. (We will treat the $1 \mathrm{~d}$ case separately, below.) Putting all this together in the usual manner, we are left with the one-loop RG equations for the interactions between electrons on opposing sides of the Fermi surface,

$$
\frac{d g_{l}}{d \ell}=-\frac{1}{\pi v_{F}} g_{l}^{2}, \quad \frac{d \tilde{g}_{l}}{d \ell}=0,
$$

where $l$ refers to the appropriate Fermi surface harmonic; for the case of a circular Fermi surface in two dimensions, $l$ is simply angular momentum. (Implicit in this is the fact that odd $l$ are associated with interactions in the triplet channel while even $l$ are in the singlet channel.)

These equations describe the changes in the effective interactions upon an infinitesimal RG transformation. They can be easily integrated to obtain expressions for the scale dependent interactions. However, these equations are only valid so long as all the interactions are weak (to justify perturbation

Note the nonrenormalization of $\lambda$ for $\Omega>\omega_{D}$. theory) and so long as $\Omega \gg \omega_{D}$. Assuming that it is the second condition that is violated first, we can obtain expression for the effective interactions at this scale by integrating to the point at which $\Omega=\omega_{D}$; the result is

$$
\mu\left(\omega_{D}\right)=\frac{\mu_{0}}{1+\mu_{0} \log \left(\Omega_{0} / \omega_{D}\right)}, \lambda\left(\omega_{D}\right)=\lambda_{0},
$$


where $\mu=g / \pi v_{F}, \lambda=\tilde{g} / \pi v_{F}$, the symmetry labels on $g$ and $\tilde{g}$ are left implicit, and the subscript " 0 " refers to the initial values of the couplings at a microscopic scale, $\Omega_{0} \sim E_{F}$.

The fact that the retarded interactions do not renormalize is certainly as noteworthy as the famous renormalization of $\mu$. This means that it is possible to estimate $\lambda$ from microscopic calculations or from high temperature measurements, such as resistivity measurements in the quasi-classical regime where $\rho \propto \lambda T$.

Once the scale $\Omega=\omega_{D}$ is reached, a new RG procedure must be adopted. At this point, the retarded and instantaneous interactions are not distinguishable, so we must simply add them to obtain a new, effective interaction, $g^{\text {eff }}\left(\omega_{D}\right)=g\left(\omega_{D}\right)+\tilde{g}$, which upon further reduction of $\Omega$ renormalizes as a nonretarded interaction. If $g^{\text {eff }}\left(\omega_{D}\right)$ is repulsive, it will be further reduced with decreasing $\Omega$. However, if it is attractive in any channel, the RG flows carry the system to stronger couplings, and eventually the perturbation theory breaks down. We can estimate the characteristic energy scale at which this breakdown occurs by integrating the one loop equations until the running coupling constant reaches a certain finite value $-1 / \alpha$ :

$$
\Omega_{1}=\omega_{D} e^{\alpha} \exp \left[-1 /\left|g^{e f f}\left(\omega_{D}\right)\right|\right] .
$$

Of course, the RG approach does not tell us how to interpret this energy scale, other than that it is the scale at which Fermi liquid behavior breaks down. However, we know on other grounds that this scale is the pairing scale, and that the breakdown of Fermi liquid behavior is associated with the onset of superconducting behavior.

\subsection{Perturbative RG approach in $D=1$}

The one loop beta function In one dimension, the structure of the perturbative beta function is very different from in higher dimensions. In addition to the familiar logarithmic divergences in the particle-particle (or Cooper) channel, there appear similar logarithms in the particle-hole channel. That these lead to a serious breakdown of Fermi liquid theory can be deduced directly from the perturbation theory, although it is only through the magic of bosonization (discussed in Section 5) that it is possible to understand what these divergences lead to.

To highlight the differences with the higher dimensional case, we will treat the $1 \mathrm{~d}$ case using the perturbative RG approach, but now taking into account the dimension specific interference between the Cooper and particlehole channels. However, having belabored the derivation of the perturbative beta function for the higher dimensional case, we will simply write down the result for the $1 \mathrm{~d}$ case; the reader interested in the details of the derivation is referred to Refs. 294 and 295.

In $1 \mathrm{~d}$, there are only two potentially important momentum transfers which scatter electrons at the Fermi surface, as contrasted with the continuum of 
possibilities in high dimension. It is conventional to indicate by $g_{1}$ the interaction with momentum transfer $2 k_{F}$, and by $g_{2}$ that with zero momentum transfer. If we are interested in the case of a nearly half filled band, we also need to keep track of the umklapp scattering, $g_{3}$, which involves a momentum transfer $2 \pi$ to the lattice (see Section 5). Consequently, we must introduce a chemical potential, $\mu$, defined such that $\mu=0$ corresponds to the half filled band. Finally, we consider the retarded interactions, $\tilde{g}_{1}, \tilde{g}_{2}$, and $\tilde{g}_{3}$ which operate at frequencies less than $\omega_{D}$. For simplicity, we consider only the case of spin rotationally invariant interactions.

The one loop RG equations (obtained by evaluating precisely the diagrams in Fig. 25), under conditions $\Omega \gg \omega_{D}, \mu$, are

$$
\begin{aligned}
& \frac{d g_{1}}{d \ell}=-\frac{g_{1}^{2}}{\pi v_{F}}, \quad \frac{d g_{c}}{d \ell}=-\frac{g_{3}^{2}}{\pi v_{F}}, \quad \frac{d g_{3}}{d \ell}=-\frac{g_{3} g_{c}}{\pi v_{F}}, \\
& \frac{d \tilde{g}_{ \pm}}{d \ell}=-\frac{g_{ \pm}}{\pi v_{F}}\left[\frac{3}{2} g_{1} \pm g_{3}+\frac{1}{2} g_{c}+\tilde{g}_{ \pm}\right], \quad \frac{d \tilde{g}_{2}}{d \ell}=0, \\
& \frac{d \mu}{d \ell}=\mu, \quad \frac{d \omega_{D}}{d \ell}=\left[1+\frac{\tilde{g}_{+}}{\pi v_{F}}\right] \omega_{D},
\end{aligned}
$$

The electron-phonon interaction in a non-Fermi liquid can be strongly renormalized. where $g_{c} \equiv g_{1}-2 g_{2}$ and $\tilde{g}_{ \pm}=\tilde{g}_{1} \pm \tilde{g}_{3}$. For $\mu \gg \Omega \gg \omega_{D}$, the same equations apply, except now we must set $g_{3}=\tilde{g}_{3}=0$. And, of course, if $\omega_{D}>\Omega$, we simply drop the notion of retarded interactions, altogether.

There are many remarkable qualitative aspects to these equations, many of which differ markedly from the analogous equations in higher dimensions. The most obvious feature is that the retarded interactions are strongly renormalized, even when the states being eliminated have energies large compared to $\omega_{D}$. What this means is that in one dimension, the effective electronphonon interaction at low energies is not simply related to the microscopic interaction strength. Some of the effects of this strong coupling on the spectral properties of quasi-one dimensional systems can be found in Refs. 295 297.

Away from half filling To see how this works out, let us consider the typical case in which the nonretarded interactions are repulsive $\left(g_{1}\right.$, and $\left.g_{2}>0\right)$ and the retarded interactions are attractive $\left(\tilde{g}_{ \pm}<0\right)$ and strongly retarded, $\omega_{D} / E_{F} \ll 1$. Far from half filling, we can also set $g_{3}=\tilde{g}_{3}=0$. The presence or absence of a spin gap is determined by the sign of $g_{1}$. Thus, just as in the $3 \mathrm{~d}$ case, in order to derive the effective theory with nonretarded interactions which is appropriate to study the low energy physics at scales small compared to $\omega_{D}$, we integrate out the fermionic degrees of freedom at scales between $E_{F}$ and $\omega_{D}$, and then compute the effective backscattering interaction,

$$
g_{1}^{e f f}=g_{1}\left(\omega_{D}\right)+\tilde{g}_{1}\left(\omega_{D}\right) .
$$

If $g_{1}^{\text {eff }}>0$ (i.e. if $g_{1}\left(\omega_{D}\right)>\left|\tilde{g}_{1}\left(\omega_{D}\right)\right|$ ), then the Luttinger liquid is a stable fixed point, and in particular no spin gap develops. If $g_{1}^{\text {eff }}<0$, however, the 
Luttinger liquid fixed point is unstable; now, the system flows to a LutherEmery fixed point with a spin gap which can be determined in the familiar way to be

$$
\Delta_{s} \sim \omega_{D} \exp \left[-\pi v_{F} / g_{1}^{e f f}\right]
$$

This looks very much like the BCS result from high dimensions. The parallel with BCS theory goes even a bit further, since under the RG transformation, a repulsive $g_{1}$ scales to weaker values in just the same way as the Coulomb pseudopotential in higher dimensions:

$$
g_{1}\left(\omega_{D}\right)=\frac{g_{1}^{0}}{1+\left(g_{1}^{0} / \pi v_{F}\right) \log \left(E_{F} / \omega_{D}\right)},
$$

where $g_{1}^{0} \equiv g_{1}\left(E_{F}\right)$. However, in contrast to the higher dimensional case, $\tilde{g}_{1}$ is strongly renormalized; integrating the one-loop equations, it is easy to show that

$$
\begin{aligned}
\tilde{g}_{1}\left(\omega_{D}\right) & =\left(\frac{\tilde{g}_{1}^{0}}{1+\tilde{g}_{1}^{0} L}\right)\left(\frac{g_{1}\left(\omega_{D}\right)}{g_{1}^{0}}\right)^{3 / 2}\left(\frac{E_{F}}{\omega_{D}}\right)^{-g_{c} / 2 \pi v_{F}}, \\
L & =\int_{0}^{\log \left(E_{F} / \omega_{D}\right)} \frac{d x}{\pi v_{F}} \frac{\exp \left[-g_{c} x / 2 \pi v_{F}\right]}{\left[1+\left(g_{1}^{0} / \pi v_{F}\right) x\right]^{3 / 2}} .
\end{aligned}
$$

Various limits of this expression can easily be analyzed - we will not give an exhaustive analysis here. For $g_{1}=g_{c}=0$, Eq. (99) reduces to the same logarithmic expression, Eq. (97), as for $g_{1}$, although because $\tilde{g}_{1}$ has the opposite sign, the result is a logarithmic increase of the effective interaction; this is simply the familiar Peierls renormalization of the electron-phonon interaction. For $g_{c}<0$, this renormalization is substantially amplified. Thus, in marked contrast to the higher dimensional case, strong repulsive interactions actually enhance the effects of weak retarded attractions!

Finally, there is bad news as well as good news. As discussed in Section 5 , the behavior of the charge modes is largely determined by the "charge Luttinger exponent, $K_{c}$, which is in turn determined by the effective interaction

$$
g_{c}^{e f f}=g_{c}+\tilde{g}_{1}^{e f f}-2 \tilde{g}_{2},
$$

according to the relation (See Eq. (16).)

$$
K_{c}=\sqrt{\frac{1+\left(g_{c}^{e f f} / \pi v_{F}\right)}{1-\left(g_{c}^{e f f} / \pi v_{F}\right)}} .
$$

In particular, the relative strength of the superconducting and CDW fluctuations are determined by $K_{c}$; the smaller $K_{c}$, i.e. the more negative $g_{c}^{\text {eff }}$, the more dominant are the CDW fluctuations. It therefore follows from Eq. (100) that a large negative value of $\tilde{g}_{1}^{\text {eff }}$ due to the renormalization of the electron-phonon interaction only throws the balance more strongly in favor of the CDW order. For this reason, most quasi 1D systems with a spin gap are CDW insulators, rather than superconductors.

Repulsive interactions enhance the effects of weak retarded attractions. 
Half filling Near half filling, the interference between the retarded and instantaneous interactions becomes even stronger. In the presence of Umklapp scattering, an initially negative $g_{c}$ renormalizes to stronger coupling, as does $g_{3}$ itself. Without loss of generality, we can take $g_{3}>0$ since its sign can be reversed by a change of basis. Then we can see that both $g_{3}$ and $g_{c}$ contribute to an inflationary growth of $\tilde{g}_{-}$. The RG equations have been integrated in Ref. 298, and we will not repeat the analysis here. The point is that all the effects discussed above apply still more strongly near half filling. In addition, we now encounter an entirely novel phenomenon - we find that the effective electron-phonon interaction strength at energy scale $\omega_{D}$ is strongly doping dependent, as well. It is possible [298], as indeed seems to be the case in the model conducting polymer polyacetylene, for the electron-phonon coupling to be sufficiently strong to open a Peierls gap of magnitude $2 \mathrm{eV}$ (roughly, $1 / 5$ of the $\pi$-band width) at half filling, and yet be so weak at a microscopic scale that for doping concentrations greater than $5 \%$, no sign of a Peierls gap

The electron-phonon coupling can even be strongly doping dependent. is seen down to temperatures of order $1 \mathrm{~K}$ !

How many of the features seen from this study of the 1DEG are specific to one dimensional systems is not presently clear. Conversely, these results prove by example that familiar properties of Fermi liquids cannot be taken as generic. In particular, strongly energy and doping dependent electron phonon interactions are certainly possibilities that should be taken seriously in systems that are not Fermi liquids.

\section{Lessons from Strong Coupling}

In certain special cases, well controlled analytic results can be obtained in the limit in which the bare electron-electron interactions are nonperturbative. We discuss several such models.

\subsection{The Holstein model of interacting electrons and phonons}

The simplest model of strong electron-phonon coupling is the Holstein model of an optic phonon, treated as an Einstein oscillator, coupled to a single tight binding electron band,

$$
H_{H o l}=-t \sum_{<i, j>, \sigma}\left[c_{i, \sigma}^{\dagger} c_{j, \sigma}+\text { H.C. }\right]+\alpha \sum_{j} x_{j} \hat{n}_{j}+\sum_{j}\left[\frac{P_{j}^{2}}{2 M}+\frac{K x_{j}^{2}}{2}\right],
$$

where $\hat{n}_{j}=\sum_{\sigma} c_{j, \sigma}^{\dagger} c_{j, \sigma}$ is the electron density operator and $P_{j}$ is the momentum conjugate to $x_{j}$.

In treating the interesting strong coupling physics of this problem, it is sometimes useful to transform this model so that the phonon displacements 
are defined relative to their instantaneous ground state configuration. This is done by means of the unitary transformation,

$$
U=\prod_{j} \exp \left[i(\alpha / K) P_{j} \hat{n}_{j}\right]
$$

which shifts the origin of oscillation as $U^{\dagger} x_{j} U=x_{j}-(\alpha / K) \hat{n}_{j}$. Consequently, the transformed Hamiltonian has the form

$U^{\dagger} H_{H o l} U=-t \sum_{<i, j>, \sigma}\left[\hat{S}_{i j} c_{i, \sigma}^{\dagger} c_{j, \sigma}+\right.$ H.C. $]-\frac{U_{e f f}}{2} \sum_{j}\left[\hat{n}_{j}\right]^{2}+\sum_{j}\left[\frac{P_{j}^{2}}{2 M}+\frac{K x_{j}^{2}}{2}\right]$,

where $\hat{S}_{i, j}=\exp \left[-i(\alpha / K)\left(P_{i}-P_{j}\right)\right]$ and $U_{e f f}=\alpha^{2} / K$.

There are several limits in which this model can be readily analyzed:

Adiabatic limit: $\boldsymbol{E}_{\boldsymbol{F}} \gg \boldsymbol{\omega}_{\boldsymbol{D}}$ In the limit $t \gg \omega_{D}$, where $\omega_{D}=\sqrt{K / M}$ is the phonon frequency and for $\alpha$ not too large, this is just the sort of model considered in the weak coupling section, or any other conventional treatment of the electron-phonon problem. Here, Migdal's theorem provides us with guidance, and at least for not too strong coupling, the BCS-Eliashberg treatment discussed in Section 9 can be applied. While $U_{\text {eff }}$ is, indeed, the effective interaction which enters the BCS expression for the superconducting $T_{c}$, because the fluctuations of $P_{i}$ are large if $M$ is large, it is not useful to work with the transformed version of the Hamiltonian.

Inverse adiabatic limit; negative $\boldsymbol{U}$ Hubbard model In the inverse adiabatic limit, $M \rightarrow 0$, fluctuations of $P_{j}$ are negligible, so that $\hat{S}_{i j} \rightarrow 1$. Hence, in this limit, the Holstein model is precisely equivalent to the Hubbard model, but with an effective negative $U$. If $U_{\text {eff }} \ll t$, this is again a weak coupling model, and will yield a superconducting $T_{c}$ given by the usual BCS expression, although in this case with a prefactor proportional to $t$ rather than $\omega_{D}$.

In contrast, if $U_{\text {eff }} \gg t$, a strong coupling expansion is required. Here, we first find the (degenerate) ground states of the unperturbed model with $t=0$, and then perform perturbation theory in small $t / U_{\text {eff }}$. In the zeroth order ground states, each site is either unoccupied, or is occupied by a singlet pair of electrons. The energy of this state is $-U_{e f f} N^{e l}$, where $N^{e l}$ is the number of electrons. These states can be thought of as the states of infinite mass, hard core charge 2e bosons on the lattice. There is a gap to the first excited state of magnitude $U_{\text {eff }}$. Second order perturbation theory in the ground state manifold straightforwardly yields an effective Hamiltonian which 
is equivalent ${ }^{19}$ to a model of hard core bosons $\left(\left[b_{i}^{\dagger}, b_{j}\right]=\delta_{i, j}\right)$

$$
H_{\text {boson }}=-t_{\text {eff }} \sum_{<i, j>}\left[b_{i}^{\dagger} b_{j}+\text { H.C. }\right]+V_{\text {eff }} \sum_{<i, j>} b_{i}^{\dagger} b_{i} b_{j}^{\dagger} b_{j}+[\infty] \sum_{j} b_{j}^{\dagger} b_{j}\left[b_{j}^{\dagger} b_{j}-1\right],
$$

with nearest neighbor hopping $t_{e f f}=2 t^{2} / U_{\text {eff }}$ and nearest neighbor repulsion $V_{e f f}=2 t_{e f f}$. This effective model is applicable for energies and temperatures small compared to $U_{\text {eff }}$.

The properties of this bosonic Hamiltonian, and closely related models where additional interactions between bosons are included, have been widely studied 299, 300]. It has a large number of possible phases, including super-

Strong attractions impede coherent motion, and enhance charge ordering. conducting, crystalline, and striped or liquid crystalline phases. The equivalence between hard core bosons and spin- $1 / 2$ operators can be used to relate this model to various spin models that have been studied in their own right. However, for the present purposes, there are two clear lessons we wish to draw from this exercise. The first is that there are ordered states, in particular insulating charge ordered states, which can compete very successfully with the superconducting state in strong coupling. The second is that, even if the system does manage to achieve a superconducting ground state, the characteristic superconducting $T_{c}$ will be proportional to $t_{e f f}$, and hence to the small parameter, $t / U_{\text {eff }}$.

Large $\boldsymbol{U}_{\text {eff }}$ : bipolarons More generally, in the strong coupling limit, $U_{\text {eff }} \gg t$, a perturbative approach in powers of $t / U_{\text {eff }}$ can be undertaken, regardless of the value of $M$. Once again, the zeroth order ground states are those of charge $2 e$ hard core bosons, as in Eq. (105). However, now the phonons make a contribution to the ground state - the ground state energy is $-U_{e f f} N^{e l}+(1 / 2) \omega_{D} N$ where $N$ is the number of sites, and the gap to the first excited state is the smaller of $U_{\text {eff }}$ and $\omega_{D}$. Still, we can study the properties of the model at energies and temperatures small compared to the gap in terms of the hard core bosonic model. Now, however,

$$
\begin{aligned}
t_{\text {eff }} & =2 \frac{t^{2}}{U_{\text {eff }}} F_{+}(X), \\
V_{\text {eff }} & =4 \frac{t^{2}}{U_{\text {eff }}} F_{-}(X),
\end{aligned}
$$

where $X \equiv \frac{U_{e f f}}{\omega_{D}}$ and

$$
F_{ \pm}(X)=\int_{0}^{\infty} d t \exp \{-t-X[1 \pm \exp (-t / X)]\} .
$$

$\overline{19}$ Clearly, $\tilde{b}_{j} \equiv c_{j \uparrow} c_{j \downarrow}$ does not satisfy the same-site piece of the bosonic commutation relation, but the hard core constraint on the $b_{j}$ bosons corrects any errors introduced by neglecting this. 
This is often referred to as a model of bipolarons. In the inverse adiabatic limit, $F_{ \pm}(X) \rightarrow 1$ as $X \rightarrow 0$, and hence these expressions reduce to those of the previous subsection. However, in the adiabatic limit, $X \gg 1, F_{+}(X) \sim$ $e^{-2 X}$, so $t_{\text {eff }}$ is exponentially reduced by a Frank-Condon factor! However, $F_{-}(X) \rightarrow 1$ as $X \rightarrow \infty$, so $V_{\text {eff }}$ remains substantial. Clearly, the lessons concerning the difficulty of obtaining high temperature superconductivity from strong coupling drawn from the negative $U$ Hubbard model apply even more strongly to the case in which the phonon frequency is small. A bipolaron mechanism of superconductivity is simply impossible unless the phonon frequency is greater than or comparable to $U_{e f f}$; in the opposite limit, the exponential suppression of $t_{e f f}$ relative to the effective interactions, $V_{e f f}$, strongly suppresses the coherent Bose-condensed state, and favors various types of insulating, charge ordered states.

\subsection{Insulating quantum antiferromagnets}

We now turn to models with repulsive interactions. To begin with, we discuss the "Mott limit" of the antiferromagnetic insulating state. Here, we imagine that there is one electron per site, and such strong interactions between them that charge fluctuations can be treated petrubatively. In this limit, as is well known, the only low energy degrees of freedom involve the electron spins, and hence the problem reduces to that of an effective quantum Heisenberg antiferromagnet.

Quantum antiferromagnets in more than one dimension In recent years, there has been considerable interest $76,7,79,80,213,230,232,301,302$ in the many remarkable quantum states that can occur in quantum spin models with sufficiently strong frustration - these studies are beyond the scope of the present review. On a hypercubic lattice (probably on any simple, bipartite lattice) and in dimension 2 or greater, there is by now no doubt that even the spin $1 / 2$ model (in which quantum fluctuations are the most severe) has a Nèel ordered ground state 222. Consequently, the properties of such systems at temperatures and energies low compared to the antiferromagnetic exchange energy, $J$, are determined by the properties of interacting spin waves. This physics, in turn, is well described in terms of a simple field theory, known as the $O(3)$ nonlinear sigma model. While interesting work is still ongoing on this problem, it is in essence a solved problem, and excellent modern reviews exist 303.

In its ordered phase, the antiferromagnet has: i) gapless spin wave excitations, and ii) reduced tendency to phase ordering due to the frustration of charge motion. Since the superconducting state possesses a spin gap (or, for d-wave, a partial gap) and is characterized by the extreme coherence of

In more than one dimension, it is a solved problem.

Antiferromagnetic order is bad for superconductivity. 
A spin fluctuation exchange mechanism in a nearly antiferromagnetic electron fluid is critiqued. charge motion, it is clear that both these features of the antiferromagnet are disadvantageous for superconductivity. 20

There is a body of thought 29 32] that holds that it is possible, at sufficiently strong doping of an antiferromagnet to reach a state in which the antiferromagnetic order and the consequent low energy spin fluctuations are eliminated and electron itineracy is restored, which yet has vestiges of the high energy spin wave excitations of the parent ordered state that can serve to induce a sufficiently strong effective attraction between electrons for high temperature superconductivity. Various strong critiques of this approach have also been articulated [18. We feel that the theoretical viability of this "spin fluctuation exchange" idea has yet to be firmly established. As an example of how this could be done, one could imagine studying a two component system consisting of a planar, Heisenberg antiferromagnet coupled to a planar Fermi liquid. One would like to see that, as some well articulated measure of the strength of the antiferromagnetism is increased, the superconducting pairing scale likewise increases. If such a system could be shown to be a high temperature superconductor, it would establish the point of principal. However, it has been shown by Schrieffer [66] that Ward identities, which are ultimately related to Goldstone's theorem, imply that long wavelength spin waves cannot produce any pairing interaction at all. A model of this sort that has been analyzed in detail is the one dimensional Kondo-Heisenberg model, which is the 1D analogue of this system 304 306. This system does not exhibit significant superconducting fluctuations of any conventional kind. While there certainly does not exist a "no-go" theorem, it does not seem likely to us that an exchange of spin waves in a nearly anitferromagnetic system can ever give rise to high temperature superconductivity. ${ }^{21}$

20 There is a very interesting line of reasoning [154 which takes the opposite viewpoint: it is argued that the important point to focus on is that both the superconductor and the antiferromagnet have gapless Goldstone modes, not whether those modes are spinless or spinful. In this line of thought there is a near symmetry, which turns out to be $\mathrm{SO}(5)$, between the $d$-wave superconducting and the Néel ordered antiferromagnetic states. This is an attractive notion, but it is not clear to us precisely how this line of reasoning relates to the more microscopic considerations discussed here.

${ }^{21}$ Under circumstances in which antiferromagnetic correlations are very short ranged, it may still be possible to think of an effective attraction between electrons mediated by the exchange of very local spin excitations 31. This escapes most of the critiques discussed above - neither Ward identities nor the general incompatibility between antiferromagnetism and easy electron itineracy have any crisp meaning at short distances. By the same token, however, it is not easy to unambiguously show that such short range magnetic correlations are the origin of strong superconducting correlations in any system, despite some recent progress along these lines 307. 
Spin gap in even leg Heisenberg ladders The physics of quantum antiferromagnets in one dimension is quite different from that in higher dimension, since the ground state is not magnetically ordered. However, its general features have been well understood for many years. In particular, for spin$1 / 2$ Heisenberg ladders or cylinders with an even number of sites on a rung, quantum fluctuations result in a state with a spin gap. This is a special case of a general result [308], known as "Haldane's conjecture," that any 1D spin system with an even integer number of electrons per unit cell has a spin rotationally invariant ground state and a finite spin gap in the excitation spectrum. This conjecture has not been proven, but has been validated in many limits and there are no known exceptions 22.

The physics of interacting electrons on ladders - i.e. "fat" 1D systems, will be discussed at length below. We believe this is an important, paradigmatic system for understanding the physics of high temperature superconductivity. The fact that even the undoped (insulating) ladder has a spin gap can be interpreted as a form of incipient superconducting pairing. Where that gap is large, i.e. a substantial fraction of the exchange energy, $J$, it is reasonable to hope that doping it will lead to a conducting state which inherits from the parent insulating state this large gap, now directly interpretable as a pairing gap.

Let us start by considering an $N$ leg spin- $1 / 2$ Heisenberg model

$$
H=\sum_{<i, j>} J_{i j} \boldsymbol{S}_{i} \cdot \boldsymbol{S}_{j}
$$

where $\boldsymbol{S}_{i}$ is the spin operator on site $i$, so for $a, b, c=\{x, y, z\},\left[S_{i}^{a}, S_{j}^{b}\right]=$ $i \delta_{i j} \epsilon^{a b c} S_{i}^{c}$ and $\boldsymbol{S}_{i} \cdot \boldsymbol{S}_{i}=3 / 4$. Here, we still take the lattice to be infinite in one ("parallel") direction but of width $N$ sites in the other. At times, we will distinguish between a ladder, with open boundary conditions in the "perpendicular" direction, and a cylinder, with periodic boundary conditions in this direction. We will typically consider isotropic antiferromagnetic couplings, $J_{i j}=J>0$.

Ladders with many legs: In the limit of large $N$, it is clear that the model can be viewed as a two dimensional antiferromagnet up to a crossover scale, beyond which the asymptotic one dimensional behavior is manifest. This viewpoint was exploited by Chakravarty 309] to obtain a remarkably accurate analytic estimate of the crossover scale. His approach was to first employ the equivalence between the Heisenberg model and the quantum nonlinear sigma model. One feature of this mapping is that the thermodynamic properties of the $d$ dimensional Heisenberg model are related to a $d+1$ dimensional sigma

$\overline{22}$ One can hardly fail to notice that the Haldane conjecture is closely related to The spin gap falls exponentially with $N$.

Insulating ladders are good parents for high temperature superconductors. the conventional band structure view that insulators are systems with a gap to both charge and spin excitations due to the fact that there are an even number of electrons per unit cell and all bands are either full or empty. 
model, with an imaginary time direction which, by suitable rescaling, is precisely equivalent to any of the spatial directions. The properties of the Heisenberg model at finite temperatures are then related to the sigma model on a generalized cylinder, which is periodic in the imaginary time direction with circumference $\hbar v_{s} / T$ where $v_{s}$ is the spin wave velocity. What Chakravarty pointed out is that, through this mapping, there is an equivalence between the Heisenberg cylinder with circumference $L=N a$ at zero temperature and the infinite planar Heisenberg magnet at temperature, $T=v_{s} / L$. From the well known exponential divergence of the correlation length with decreasing temperature in the $2 \mathrm{~d}$ system, he obtained the asymptotic expression for the dimensional crossover length in the cylinder,

$$
\xi_{d i m} \sim a \exp [0.682 N] .
$$

As this estimate is obtained from the continuum theory, it is only well justified in the large $N$ limit. However, comparison with numerical experiments described in Section 11 (some of which predated the analytic theory [310]) reveal that it is amazingly accurate, even for $N=2$, and that the distinction between ladders and cylinders is not very significant, either.

This result is worth contemplating. It implies that the special physics of one dimensional magnets is only manifest at exponentially long distances in fat systems. Correspondingly, it means that these effects are confined to energies (or temperatures) smaller than the characteristic scale

$$
\Delta_{\text {dim }}=v_{s} / \xi_{\text {dim }} .
$$

As a practical matter, it means that only the very narrowest systems, with $N$ no bigger than 3 or 4 , will exhibit the peculiarities of one dimensional magnetism at any reasonable temperature.

To understand more physically what these crossover scales mean, one needs to know something about the behavior of one dimensional magnets. Since even leg ladders and cylinders have a spin gap, it is intuitively clear (and correct) that $\Delta_{\text {dim }}$ is nothing but the spin gap and $\xi_{\text {dim }}$ the correlation length associated with the exponential fall of magnetic correlations at $T=0$. For odd leg ladders, $\xi_{\text {dim }}$ is analogous to a Josephson length, where correlations crossover from the two dimensional power law behavior associated with the existence of Goldstone modes, to the peculiar quantum critical behavior of the one dimensional spin $1 / 2$ Heisenberg chain.

The two leg ladder: It is often useful in developing intuition to consider limiting cases in which the mathematics becomes trivial, although one must always be sensitive to the danger of being overly influenced by the naive intuitions that result.

In the case of the two leg ladder, there exists such a limit, $J_{\perp} \gg J_{\|}$, where $J_{\perp}$ and $J_{\|}$are, respectively, the exchange couplings across the rungs, and along the sides of the ladder. Here the zeroth order ground state is a 
direct product of singlet pairs (valence bonds) on the rungs of the ladder. Perturbative corrections to the ground state cause these valence bonds to resonant, locally, but do not fundamentally affect the character of the ground state. The ground state energy per site is

$$
E_{0}=-(3 / 8) J_{\perp}\left[1+\left(J_{\|} / J_{\perp}\right)^{2}+\ldots\right] .
$$

Since each valence bond is nothing but a singlet pair of electrons, this makes it clear that there is a very direct sense in which the two leg ladder can be thought of as a paired insulator. The lowest lying spin- 1 excited states are a superposition of bond triplets on different rungs, and have a dispersion relation which can easily be derived in perturbation theory:

$$
E_{\text {triplet }}=J_{\perp}+J_{\|} \cos (k)+\mathcal{O}\left(J_{\|}^{2} / J_{\|}\right) .
$$

This, too, reveals some features that are more general, such as a minimal spin gap of magnitude $\Delta_{s}=J_{\perp}\left[1-\left(J_{\|} / J_{\perp}\right)+\mathcal{O}\left(J_{\|}^{2} / J_{\|}^{2}\right)\right]$ at what would be the antiferromagnetic ordering wavevector $k=\pi$.

\subsection{The isolated square}

While we are considering mathematically trivial problems, it is worth taking a minute to discuss the solution of the $t-J$ model (defined in Eq. (126), below) on an isolated 4-site square. The pedagogic value of this problem, which is exactly diagonalizable, was first stressed by Trugman and Scalapino [311. This idea was recently carried further by Auerbach and collaborators [312. 313, who have attempted to build a theory of the 2D $t-J$ model by linking together fundamental squares. The main properties of the lowest energy states of this system are given in Table 2 for any number of doped holes.

The "undoped" state of this system (i.e. with 4 electrons) is a singlet with ground state energy $E_{0}=-3 J$. However, interestingly, it is not in the identity representation of the symmetry group of the problem - it is odd under $90^{\circ}$ rotation. If we number the sites of the square sequentially from 1 to 4 , then the ground state wavefunction is

$$
\mid 4-\text { electron }\rangle=\left[\hat{P}_{1,2}^{\dagger} \hat{P}_{3,4}^{\dagger}-\hat{P}_{1,4}^{\dagger} \hat{P}_{2,3}^{\dagger}\right]|0\rangle
$$

where $\hat{P}_{i, j}^{\dagger}=\hat{P}_{j, i}^{\dagger}=\left[c_{i, \uparrow}^{\dagger} c_{j, \downarrow}^{\dagger}+c_{j, \uparrow}^{\dagger} c_{i, \downarrow}^{\dagger}\right] / \sqrt{2}$ creates a singlet pair of electrons on the bond between sites $i$ and $j$. Manifestly, $\mid 4$-electron $\rangle$ has the form of an odd superposition of nearest neighbor valence bond states - in this sense, it is the quintessential resonating valence bond state. The lowest lying excitation is a spin-1 state with energy $-2 J$, so the spin gap is $J$.

There are level crossings as a function of $J / t$ in the "one hole" ( 3 electron) spectrum. For $0<J / t<(8-\sqrt{52}) / 3 \approx 0.263$ the ground state is a spin $3 / 2$ multiplet with energy $E_{1}=-2 t$. It is orbitally nondegenerate with zero 


\begin{tabular}{|c||c|c|c|}
\multicolumn{4}{c|}{0 holes } \\
\hline & Energy & Spin & Momentum \\
\hline \hline g.s. & $E=-3 J$ & $S=0$ & $P=\pi$ \\
\hline $1^{\text {st }}$ e.s. & $E=-2 J$ & $S=1$ & $P=0$ \\
\hline
\end{tabular}

1 hole

\begin{tabular}{|c||c|c|c|}
\hline & Energy & Spin & Momentum \\
\hline \hline $0<J / t<0.263$ & & & \\
\hline g.s. & $E=-2 t$ & $S=3 / 2$ & $P=0$ \\
\hline $1^{\text {st }}$ e.s. & $E=-J-\sqrt{J^{2} / 4+3 t^{2}}$ & $S=1 / 2$ & $P= \pm \pi / 2$ \\
\hline $0.263<J / t<2 / 3$ & & & \\
\hline g.s. & $E=-J-\sqrt{J^{2} / 4+3 t^{2}}$ & $S=1 / 2$ & $P= \pm \pi / 2$ \\
\hline $1^{\text {st }}$ e.s. & $E=-2 t$ & $S=3 / 2$ & $P=0$ \\
\hline \hline $2 / 3<J / t<2$ & & & \\
\hline g.s. & $E=-J-\sqrt{J^{2}} / 4+3 t^{2}$ & $S=1 / 2$ & $P= \pm \pi / 2$ \\
\hline $1^{\text {st }}$ e.s. & $E=-3 J / 2-t$ & $S=1 / 2$ & $P=\pi$ \\
\hline \hline $2<J / t$ & & & \\
\hline g.s. & $E=-3 J / 2-t$ & $S=1 / 2$ & $P=\pi$ \\
\hline $1^{\text {st }}$ e.s. & $E=-J-\sqrt{J^{2}} / 4+3 t^{2}$ & $S=1 / 2$ & $P= \pm \pi / 2$ \\
\hline
\end{tabular}

\begin{tabular}{|c|c|c|c|}
\hline \multicolumn{4}{|c|}{2 holes } \\
\hline & Energy & Spin & Momentum \\
\hline $0<J / t<2$ & & & \\
\hline g.s. & $E=-J / 2-\sqrt{J^{2} / 4+8 t^{2}}$ & $S=0$ & $P=0$ \\
\hline $1^{\text {st }}$ e.s. & $E=-2 t$ & $S=1$ & $P= \pm \pi / 2$ \\
\hline$\overline{2<J / t}$ & & & \\
\hline g.s. & $E=-J / 2-\sqrt{J^{2} / 4+8 t^{2}}$ & $S=0$ & $P=0$ \\
\hline $1^{\text {st }}$ e.s. & $E=-J$ & $S=0$ & $P=\pi, \pm \pi / 2$ \\
\hline
\end{tabular}

Table 2. The low energy spectrum of the 4 -site $t-J$ square for 0 holes ( 4 electrons), 1 hole ( 3 electrons), and 2 holes ( 2 electrons). The 3 and 4 hole problems are left as an exercise for the reader.

momentum (we consider the square as a 4 -site chain with periodic boundary conditions and refer to the momentum along the chain.) For $(8-\sqrt{52}) / 3<$ $J / t<2$ the ground state has spin $1 / 2$, is two-fold degenerate with crystal momentum $\pm \pi / 2$, and has energy $E_{1}=-\left[2 J+\sqrt{J^{2}+12 t^{2}}\right] / 2$. For $2<J / t$, the ground state has spin $1 / 2$, zero momentum, and energy $E_{1}=-3 J / 2-t$.

The two hole (2 electron) ground state has energy $E_{2}=-\left[J+\sqrt{J^{2}+32 t^{2}}\right] / 2$, and spin 0 . It lies in the identity representation of the symmetry group. The lowest excitation is a spin 1 state. For $0<J / t<2$ it has crystal momentum $k= \pm \pi / 2$ (i.e. it has a two-fold orbital and 3-fold spin degeneracy) and has 
energy $E_{2}(S=1)=-2 t$. For $2<J / t$ it is orbitally nondegenerate with energy $E_{2}(S=1)=-J$.

One important consequence of this, which follows directly from the WignerEckhart theorem, is that the pair annihilation operator that connects the zero hole and the two hole ground states must transform as $d_{x^{2}-y^{2}}$. This is, perhaps, the most important result of this exercise. It shows the robustness of the $d$ wave character of the pairing in a broad class of highly correlated systems. The dominant component of this operator is of the form

$$
\phi_{1}=\hat{P}_{12}-\hat{P}_{23}+\hat{P}_{34}-\hat{P}_{41} .
$$

It also includes terms that create holes on next nearest neighbor diagonal sites 314, 315.

There are a few other interesting aspects of this solution. In the single hole sector, the ground state is maximally polarized, in agreement with Nagaoka's theorem, for sufficiently large $t / J$, but there is a level crossing to a state with smaller spin when $t / J$ is still moderately large. Moreover, even when the single hole state is maximally polarized, the two hole state, like the zero hole state, is always a spin singlet. Both of these features have been observed in numerical studies on larger $t-J$ clusters 316.

If we look still more closely at the $J / t \rightarrow 0$ limit, there is another interesting aspect of the physics: It is intuitively clear that in this limit, the holes should behave as spinless fermions. This statement requires no apology in the maximum spin state. Thus, the lowest energy spin-1 state with two holes has energy $E_{2}(S=1)=-2 t$ in this limit. It corresponds to a state in which one spinless fermion has crystal momentum $k=0$ and energy $-2 t$, and the other has crystal momentum $\pm \pi / 2$ and energy 0 . However, what is more interesting is that there is also a simple interpretation of the two hole ground state in the same representation. The antisymmetry of the spins in their singlet state means that they affect the hole dynamics through a Berry's phase as if half a magnetic flux quantum were threaded through the square. This Berry's phase implies that the spinless fermions satisfy antiperiodic boundary conditions. The ground state is thus formed by occupying the single particle states with $k= \pm \pi / 4$ for a total ground state energy of $E_{2}=-2 \sqrt{2} t$, precisely the $J / t \rightarrow 0$ limit of the expression obtained above. The interesting thing is that, in this case, it is the hole kinetic energy, and not the exchange energy, which favors the singlet over the triplet state. This simple exercise provides an intuitive motivation for the existence of various forms of "flux phase" in strongly interacting systems 317.

Finally, it is worth noting that pair binding occurs, in the sense that $2 E_{1}-E_{0}-E_{2}>0$, so long as $J / t>\sqrt{(39-\sqrt{491}) / \sqrt{3}} \approx 0.2068$. We will return to the issue of pair binding in Section 11 where we will show a similar behavior in Hubbard and $t-J$ ladders. 


\subsection{The spin gap proximity effect mechanism}

The final strong coupling model we will consider consists of two inequivalent 1DEG's weakly coupled together - a generalization of a two leg ladder. Each 1DEG is represented by an appropriate bosonized field theory - either a Luttinger liquid or a Luther-Emery liquid. Most importantly, the two systems are assumed to have substantially different values of the Fermi momentum, $k_{F}$ and $\tilde{k}_{F}$. We consider the case in which the interactions between the two systems are weak, but the interactions within each 1DEG may be arbitrarily large. The issue we address is what changes in the properties of the coupled system are induced by these interactions. (For all technical details, see Refs. 20 and 25.)

Intuitive description of the spin gap proximity effect...

... as a kinetic energy driven mechanism of pairing.
There is an important intuitive reason to expect this system to exhibit a novel form of kinetic energy driven superconducting pairing. Because $k_{F} \neq$ $\tilde{k}_{F}$, single particle tunnelling between the two 1DEG's is not a low energy process - it is irrelevant in the renormalization group sense, and can be ignored as anything but a high energy virtual fluctuation. The same conclusion holds for any weak coupling between the $2 k_{F}$ or $4 k_{F}$ density wave fluctuations. There are only two types of coupling that are potentially important at low energies: pair tunnelling, since the relevant pairs have 0 momentum, and coupling between long wavelength spin fluctuations.

The magnetic interactions are marginal to leading order in a perturbative RG analysis - they turn out to be marginally relevant if the interactions are antiferromagnetic and marginally irrelevant if ferromagnetic [304, 306]. The effect of purely magnetic interactions has been widely studied in the context of Kondo-Heisenberg chains, but will not be discussed here. The effect of triplet pair tunnelling has only been superficially analyzed in the literature [25, 318, 319 - it would be worthwhile extending this analysis, as it may provide some insight into the origin of the triplet superconductivity that has been observed recently in certain highly correlated materials. However, in the interest of brevity, we will ignore these interactions.

Singlet pair tunnelling interactions between the two 1DEG's have a scaling dimension which depends on the nature of the correlations in the decoupled system. Under appropriate circumstances, they can be relevant. When this is the case, the coupled system scales to a new strong coupling fixed point which exhibits a total spin gap and strong global superconducting fluctuations. This is what we refer to as the spin gap proximity effect, because the underlying physics is analogous to the proximity effect in conventional superconductors. The point is that even if it is energetically costly to form pairs in one or both of the 1DEGs, once the pairs are formed they can coherently tunnel between the two systems, thereby lowering their zero point kinetic energy. Under appropriate circumstances, the kinetic energy gain outweighs the cost of pairing. This mechanism is quite distinct from any relative of the BCS mechanism - it does not involve an induced attraction. 
The explicit model which is analyzed here is expressed in terms of four bosonic fields: $\phi_{c}$ and $\phi_{s}$ represent the charge and spin degrees of freedom of the first 1DEG, and $\tilde{\phi}_{c}$ and $\tilde{\phi}_{s}$ of the other, as is discussed in Section 5, above. The Hamiltonian of the decoupled system is the general bosonized Hamiltonian described in that section, with appropriate velocities and charge Luttinger exponents, $v_{s}, v_{c}, \tilde{v}_{s}, \tilde{v}_{c}, K_{c}$, and $\tilde{K}_{c}$ if both are Luttinger liquids, and values of the spin gap, $\Delta_{s}$ and $\tilde{\Delta}_{s}$ in the case of Luther-Emery liquids (i.e. if the cosine potential in the sine-Gordon theory for the spin degrees of freedom is relevant). If we ignore the long wavelength magnetic couplings and triplet pair tunnelling between the two systems, the remaining possibly important interactions at low energy,

$$
H_{\text {inter }}=\int d x\left[\mathcal{H}_{\text {for }}+\mathcal{H}_{\text {pair }}\right]
$$

are the forward scattering (density-density and current-current) interactions in the charge sector

$$
\mathcal{H}_{\text {for }}=V_{1} \partial_{x} \phi_{c} \partial_{x} \tilde{\phi}_{c}+V_{2} \partial_{x} \theta_{c} \partial_{x} \tilde{\theta}_{c}
$$

where $\theta$ designates the field dual to $\phi$ (see Section 5), and the singlet pair tunnelling

$$
\mathcal{H}_{\text {pair }}=\mathcal{J} \cos \left[\sqrt{2 \pi} \phi_{s}\right] \cos \left[\sqrt{2 \pi} \tilde{\phi}_{s}\right] \cos \left[\sqrt{2 \pi}\left(\theta_{c}-\tilde{\theta}_{c}\right)\right] .
$$

As discussed previously, the singlet pair creation operator involves both the spin and the charge fields.

The forward scattering interactions are precisely marginal, and should properly be incorporated in the definition of the fixed point Hamiltonian. $\mathcal{H}_{\text {pair }}$ is a nonlinear interaction; the coupled problem with nonzero $\mathcal{J}$ has not been exactly solved. However, it is relatively straightforward to asses the perturbative relevance of this interaction, and to deduce the properties of the most likely strong coupling fixed point (large $\mathcal{J}$ ) which governs the low energy physics when it is relevant.

The general expression for the scaling dimension of $\mathcal{H}_{\text {pair }}$ is a complicated analytic combination of the parameters of the decoupled problem

$$
\delta_{\text {pair }}=\frac{1}{2}\left[\frac{A}{K_{c}}+\frac{B}{\tilde{K}_{c}}+K_{s}+\tilde{K}_{s}\right]
$$

where $A=1$ and $B=1$ in the absence of intersystem forward scattering interactions, but more generally $A$ and $B$ are complicated functions of the coupling constants. For illustrative purposes, one can consider the explicit expression for these functions under the special circumstances $V_{2}=-\left(\tilde{v}_{c} / v_{c}\right)\left(K_{c} \tilde{K}_{c}\right) V_{1}$; then $A=\sqrt{1-\left(V_{1}^{2} K_{c} \tilde{K}_{c} / v_{c} \tilde{v}_{c}\right)}$ and $B=\left(v_{c}-V_{1} K_{c}\right)^{2} / \sqrt{v_{c}^{4}-V_{1}^{2} v_{c} \tilde{v}_{c} K_{c} \tilde{K}_{c}}$. The scaling dimension of the pair tunnelling interaction is introduced. 
The physical effects which make pair tunnelling relevant are described.

The implications of strong pair tunnelling are discussed.

Numerical studies are motivated... that $K_{s}=\tilde{K}_{s}=1$. If one or the other 1DEG is a Luther-Emery liquid, one should substitute $K_{s}=0$ or $\tilde{K}_{s}=0$ in the above expression.

Pair tunnelling is perturbatively relevant if $\delta_{\text {pair }}<2$, and irrelevant otherwise. Clearly, having a preexisting spin gap in either of the 1DEG's dramatically decreases $\delta_{\text {pair }}$ - if there is already pairing in one subsystem, then it stands to reason that pair tunnelling will more easily produce pairing in the other. However, even if neither system has a preexisting spin gap, there are a wide set of physical circumstances for which $\delta_{\text {pair }}<2$. Notice, in particular, that repulsive intersystem interactions, $V_{1}>0$, produce a reduction of $\delta_{\text {pair }}$. Again, the physics of this is intuitive - an induced anticorrelation between regions of higher than average electron density in the two 1DEG's means that where there is a pair in one system, there tends to be a low density region on the other which is just waiting for a pair to tunnel into it. (See, also, Section 6.)

In the limit that $\mathcal{J}$ is large, the spin fields in both 1DEG's are locked, which implies a total spin gap, and the out-of-phase fluctuations of the dual charge phases are gapped as well. This means that the only possible gapless modes of the system involve the total charge phase, $\phi \equiv\left[\phi_{c}+\tilde{\phi}_{c}\right] / \sqrt{2}$, and its dual, $\theta \equiv\left[\theta_{c}+\tilde{\theta}_{c}\right] / \sqrt{2}$. $\theta$ is simply the total superconducting phase of the coupled system, and $\phi$ the total CDW phase. At the end of the day, this strong coupling fixed point of the coupled system is a Luther-Emery liquid, and consequently has a strong tendency to superconductivity. In general, there will be substantial renormalization of the effective parameters as the system scales from the weak to the strong coupling fixed point. Thus, it is difficult to estimate the effective Luttinger parameters which govern the charge modes of the resulting Luther-Emery liquid. A naive estimate, which may well be unreliable, can be be made by simply setting $\mathcal{J} \rightarrow \infty$. In this case, all the induced gaps are infinite, and the velocity and Luttinger exponent that govern the dynamics of the remaining mode are

$$
\begin{aligned}
K_{c}^{\text {total }} & =\sqrt{\frac{v_{c} K_{c}+\tilde{v}_{c} \tilde{K}_{c}+2 V_{2}}{v_{c} / K_{c}+\tilde{v}_{c} / \tilde{K}_{c}+2 V_{1}}}, \\
v_{c}^{\text {total }} & =\frac{1}{4} \sqrt{\left[v_{c} K_{c}+\tilde{v}_{c} \tilde{K}_{c}+2 V_{2}\right]\left[v_{c} / K_{c}+\tilde{v}_{c} / \tilde{K}_{c}+2 V_{1}\right]} .
\end{aligned}
$$

\section{Lessons from Numerical Studies of Hubbard and Related Models}

High temperature superconductivity is a result of strong electronic correlations. Couple this prevailing thesis with the lack of controlled analytic methods for most relevant models, and the strong motivation for numerical approaches becomes evident. Such numerical studies are limited to relatively small systems, due to a rapid growth in complexity with system size. However, 
many of the interesting aspects of the high temperature superconductors, especially those which relate to the "mechanism" of pairing, are moderately local, involving physics on the length scale of the superconducting coherence length $\xi$. Since $\xi$ is typically a few lattice spacings in the high $T_{c}$ compounds, one expects that numerical solutions of model problems on clusters with as few as 50-100 sites should be able to reveal the salient features of high temperature superconductivity, if it exists in these models. Moreover, numerical studies can guide our mesoscale intuition, and serve as important tests of analytic predictions.

Notwithstanding these merits, a few words of caution are in order. Even the largest systems that have been studied so far ${ }^{23}$ are still relatively small. Therefore, the results are manifestly sensitive to the shape and size of the cluster and other finite size effects. Some features, especially with regard to stripes, appear particularly sensitive to small changes in the model such as the presence of second neighbor hopping, 323, 324, the type of boundary conditions 325], etc. Less subtle modifications seem to have important consequences, too 328], most notably the inclusion of long range Coulomb forces (although this has been much less studied). This sensitivity has resulted in considerable controversy in the field concerning the true ground state phase diagrams of the stated models in the thermodynamic limit; see Refs. 325327 and 329, among others.

The best numerical data, especially in terms of system size, exists for narrow Hubbard and $t-J$ ladders. We therefore begin by considering them. Apart from their intrinsic appeal, these systems also offer several lessons which we believe are pertinent to the two dimensional models. The second part of this section provides a brief review of the conflicting results and views which have emerged from attempts to extrapolate from fat ladders and small periodic clusters to the entire plane.

We feel that numerical studies are essential in order to explore the important mesoscale physics of highly correlated systems, but except in the few cases where a careful finite size scaling analysis has been possible over a wide range of system sizes, conclusions concerning the long distance physics should be viewed as speculative. Even where the extrapolation to the thermodynamic limit has been convincingly established for a given model, the established fact that there are so many closely competing phases in the strong correlation limit carries with it the corollary that small changes in the Hamiltonian can sometimes tip the balance one way or the other. Thus, there are significant limitations concerning the conclusions that can be drawn from numerical studies. In the present section we focus on the reproducible features of the local correlations that follow robustly from the physics of strong, short ranged repulsions between electrons, paying somewhat less attention to

$\overline{23}$ The largest are about 250 sites [320,321] using the density matrix renormalization group method (DMRG) and up to approximately 800 sites in Green function Monte Carlo simulations. 322

What do we learn from numerical studies? 
the various controversies concerning the actual phase diagram of this or that model.

We entirely omit any discussion of the technical details of the numerical calculations. Methods that have been used include exact diagonalization by Lanczos techniques, Monte Carlo simulations of various sorts, numerical renormalization group approaches, and variational ansatz. The reader who is interested in such aspects is invited to consult Refs. 330 335.

\subsection{Properties of doped ladders}

Ladder systems, that is, quasi-one dimensional systems obtained by assembling chains one next to the other, constitute a bridge between the essentially understood behavior of strictly one dimensional models and the incompletely understood behavior in two dimensions. Such systems are not merely a theoretical creation but are realized in nature [336, 337]. For example, two leg $S=1 / 2$ ladders (two coupled spin- $1 / 2$ chains) are found in vanadyl pyrophosphate $(\mathrm{VO})_{2} \mathrm{P}_{2} \mathrm{O}_{7}$. Similarly, the cuprate compounds $\mathrm{SrCu}_{2} \mathrm{O}_{3}$ and $\mathrm{Sr}_{2} \mathrm{Cu}_{3} \mathrm{O}_{5}$ consist of weakly coupled arrays of 2-leg and 3-leg ladders, respectively. It is likely that ladder physics is also relevant to the high temperature superconductors, at least in the underdoped regime, where ample experimental evidence exists for the formation of self-organized stripes.

In this section we review some of the most prominent features of Hubbard and especially $t-J$ ladders. As we shall see the data offers extensive support in favor of the contention that a purely electronic mechanism of superconductivity requires mesoscale structure [14]. Specifically, we will find that spin gap formation and pairing correlations, with robust $d$-wave-like character, are intimately connected. Both of these signatures of local superconductivity appear as distinct and universal features in the physics of doped ladders. Nevertheless, they tend to diminish, in some cases very rapidly, with the lateral extent of the ladder, thus strongly suggesting that such structures are essential for the attainment of high temperature superconductivity. In addition we shall demonstrate the tendency of these systems to develop charge density wave correlations upon doping; it is natural to imagine that as the transverse width of the ladder tends to infinity, these density wave correlations will evolve into true two dimensional stripe order.

\section{Spin gap and pairing correlations}

Hubbard chains: The purely one dimensional Hubbard model can be solved exactly using Bethe ansatz 338, 339 and thus may seem out of place in this section. However, like other models in this section, it is a lattice fermion model. In analyzing it, we will encounter many of the concepts that will figure prominently in our discussion of the other models treated here, most notably the importance of intermediate scales. Anyway, in many cases, the 
Bethe ansatz equations themselves must be solved numerically, so we can view this as simply a more efficient numerical algorithm which permits us to study larger systems (up to 1000 sites [14] or more).

The Hubbard Hamiltonian is

$$
H_{U}=-t \sum_{\langle i, j\rangle, s}\left(c_{i, s}^{\dagger} c_{j, s}+\text { h.c. }\right)+U \sum_{i} n_{i, \uparrow} n_{i, \downarrow},
$$

where \langle\rangle denotes nearest neighbors on a ring with an even number of sites $N$ and $N+Q$ electrons. We define $E(Q, S)$ to be the lowest lying energy eigenvalue with total spin $S$ and "charge" $Q$. Whenever the ground state is a spin singlet we can define the spin gap $\Delta_{s}$ as the energy gap to the lowest $S=1$ excitation

$$
\Delta_{s}(Q)=E(Q, 1)-E(Q, 0) .
$$

The pair binding energy is defined as

$$
E_{p b}(Q)=2 E(Q+1)-E(Q+2)-E(Q),
$$

where $E(Q)$ has been minimized with respect to $S$. A positive pair binding energy means that given $2(N+Q+1)$ electrons and two clusters, it is energetically more favorable to place $N+Q+2$ electrons on one cluster and $N+Q$ on the other than it is to put $N+Q+1$ electrons on each cluster. In this sense, a positive $E_{p b}$ signifies an effective attraction between electrons. The exact particle-hole symmetry of the Hubbard model on a bipartite lattice implies that electron doping $Q>0$ is equivalent to hole doping $Q<0$.

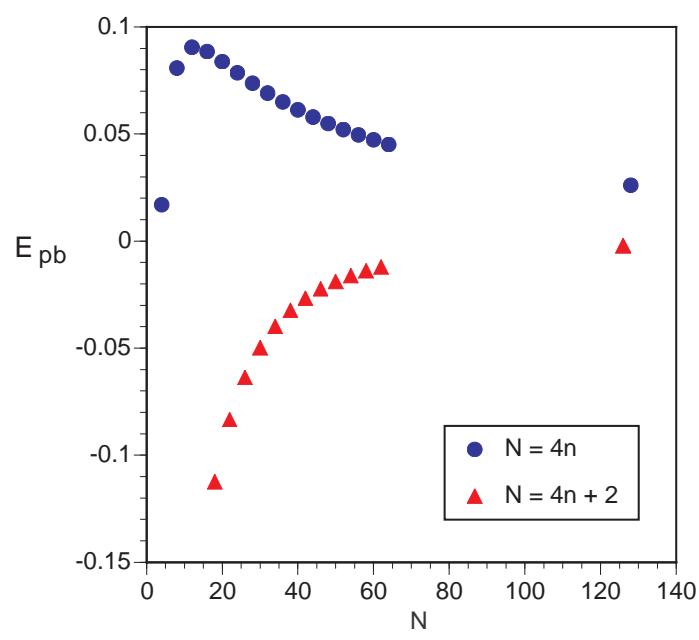

Fig. 26. Pair binding energy, $E_{p b}$, of $N=4 n$ and $N=4 n+2$ site Hubbard rings with $t=1$ and $U=4$. (From Chakravarty and Kivelson. [14]) 
Fig. 26 displays the pair binding energy for electrons added to $Q=0$ rings. The role of intermediate scales is apparent: $E_{p b}$ vanishes for large $N$ and is maximal at an intermediate value of $N$. (The fact that pair binding occurs for $N=4 n$ rings but not when $N=4 n+2$ is readily understood from low order perturbation theory in $U / t$ [14]). Moreover, the spin gap $\Delta_{s}$ reaches a maximum at intermediate interaction strength, and then decreases for large values of $U$, as expected from its proportionality to the exchange constant $J=4 t^{2} / U$ in this limit. The pair binding energy $E_{p b}$ follows suit with a similar dependence, as seen from Fig. 27.

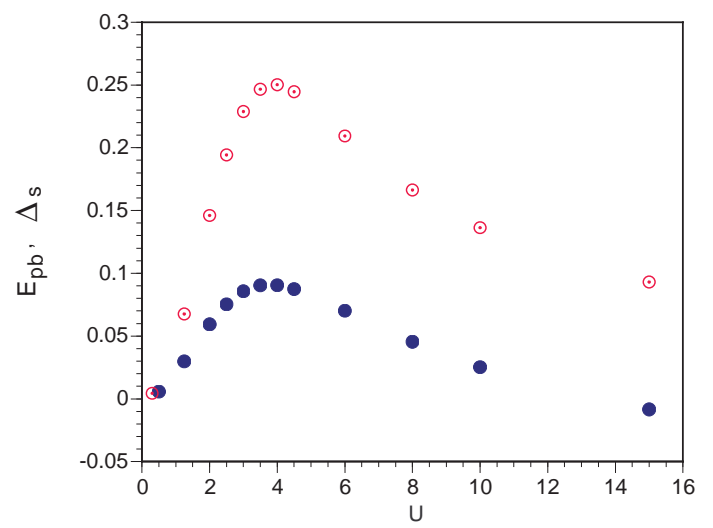

Fig. 27. Pair binding energy, $E_{p b}$ (solid symbols), and spin gap, $\Delta_{s}$ (open symbols), of a 12 site $Q=0$ Hubbard ring as a function of $U$ in units of $t=1$. (From Chakravarty and Kivelson. 14, )

We have already seen the intimate relation between the spin gap and the superconducting susceptibility in the context of quasi-one dimensional superconductors (see Section 5). Further understanding of the relation between pair binding and the spin gap can be gained by using bosonization to study the Hubbard model in the large $N$ limit [14, 339]. The result for $N=4 n \gg 1$ is

$$
\begin{aligned}
\Delta_{s} & =\frac{v_{s}}{N}\left[B_{1} \ln ^{1 / 2}(N)+B_{2}\right]+\ldots \\
E_{p b} & =\Delta_{s}+B_{3} \frac{v_{s}}{N}-\frac{B_{4}}{N^{2}}\left[\frac{v_{c}^{2}}{\Delta_{c}}\right]+\ldots
\end{aligned}
$$

The spin gap and Here, $v_{s}$ and $v_{c}$ are the spin and charge velocities, respectively (in units in pairing are related. which the lattice constant is unity), and $\Delta_{c}$ is the charge gap in the $N \rightarrow \infty$
Intermediate scales play an important role. limit. The constants, $B_{j}$, are numbers of order unity. The important lesson of this analysis is that pair binding is closely related to the phenomenon of spin gap formation. Indeed, for large $N, E_{p b} \approx \Delta_{s}$. 
Hubbard and $t-J$ ladders: In the thermodynamic limit, where the number of sites $N \rightarrow \infty$, Hubbard chains, and their strong coupling descendants the $t-J$ chains, have no spin gap and a small superconducting susceptibility, irrespective of the doping level. In contrast, ladder systems can exhibit both a spin gap and a strong tendency towards superconducting order even in the thermodynamic limit. While these systems are infinite in extent, the mesoscopic physics comes in through the finiteness of the transverse dimension.

In the large $U$ limit and at half filling (one electron per site) the Hubbard ladder is equivalent to the spin- $1 / 2$ Heisenberg ladder

$$
H_{J}=J \sum_{\langle i, j\rangle} \mathbf{S}_{i} \cdot \mathbf{S}_{j},
$$

where $\mathbf{S}_{i}$ is a spin $1 / 2$ operator, $J=4 t^{2} / U \ll t$ is the antiferromagnetic exchange interaction, and $\langle i, j\rangle$ now signifies nearest neighbor sites of spacing $a$ on the ladder. As discussed in Section 10, there is a marked difference between the behavior of ladders with even and odd numbers of chains or "legs". While even leg ladders are spin gapped with exponentially decaying spin-spin correlations, odd leg ladders are gapless and exhibit power law falloff of these correlations (up to logarithmic corrections). This difference is clearly demonstrated in Fig. 28. The spin gaps for the first few even leg ladders are known numerically [310, 340].

For the two, four, and six leg ladders, $\Delta_{s}=0.51(1) J, \Delta_{s}=0.17(1) J$, and $\Delta_{s}=0.05(1) J$, respectively. This gap appears to vanish exponentially with the width $W$ of the system, in accordance with the theoretical estimate [309] $\Delta_{s} \sim 3.35 J \exp [-0.682(W / a)]$, as discussed in Section 10. Although odd leg Heisenberg ladders are gapless, they are characterized by an energy scale which has the same functional dependence on $W$ as $\Delta_{s}$. Below this energy, the excitations are gapless spinons analogous to those in the Heisenberg chain [309], while above it they are weakly interacting spin waves. Based on our experience with the Hubbard rings we expect that spin gap formation is related to superconductivity. As we shall see below this is indeed the case once the ladders are doped with holes. On the face of it, this implies that only rather narrow ladders are good candidates for the mesoscopic building blocks of a high temperature superconductors.

When the Hubbard ladder is doped with holes away from half filling, its strong coupling description is modified from the Heisenberg model (Eq. (125)) to the $t-J$ model

$$
H_{t-J}=-t \sum_{\langle i, j\rangle, s}\left(c_{i, s}^{\dagger} c_{j, s}+h . c .\right)+J \sum_{\langle i, j\rangle}\left[\mathbf{S}_{i} \cdot \mathbf{S}_{j}-\frac{1}{4} n_{i} n_{j}\right],
$$

which is defined with the supplementary constraint of no doubly occupied sites. This is the version which has been most extensively studied numerically. Unless otherwise stated, we will quote results for representative values of $J / t$ in the range $J / t=0.35$ to 0.5 .

The number of legs matters!

Widening the ladder closes the gap. 


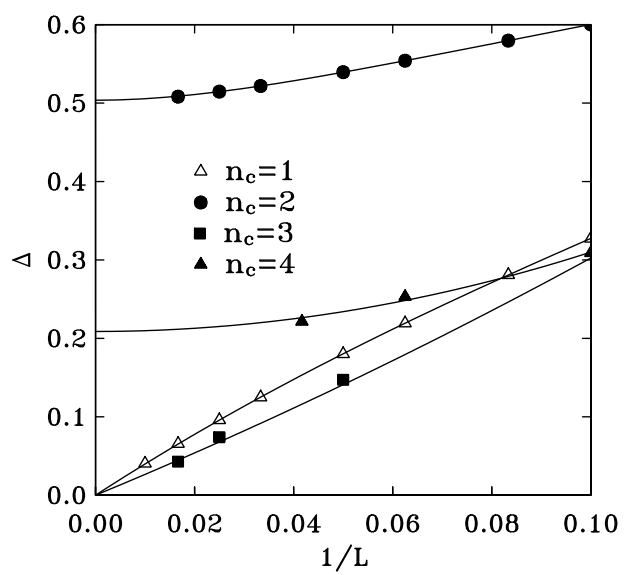

Fig. 28. Spin gaps as a function of system size $L$ for open $L \times n_{c}$ Heisenberg ladders. (From White et al. 310)

Numerical studies of the two leg Hubbard model 341, 342 have demonstrated that doping tends to decrease the spin gap continuously from its value in the undoped system but it persists down to at least an average filling of $\langle n\rangle=0.75$, as can be seen from the inset in Fig. 29. A similar behavior is observed in the $t-J$ ladder although the precise evolution of the spin gap upon doping depends on details of the model [323].

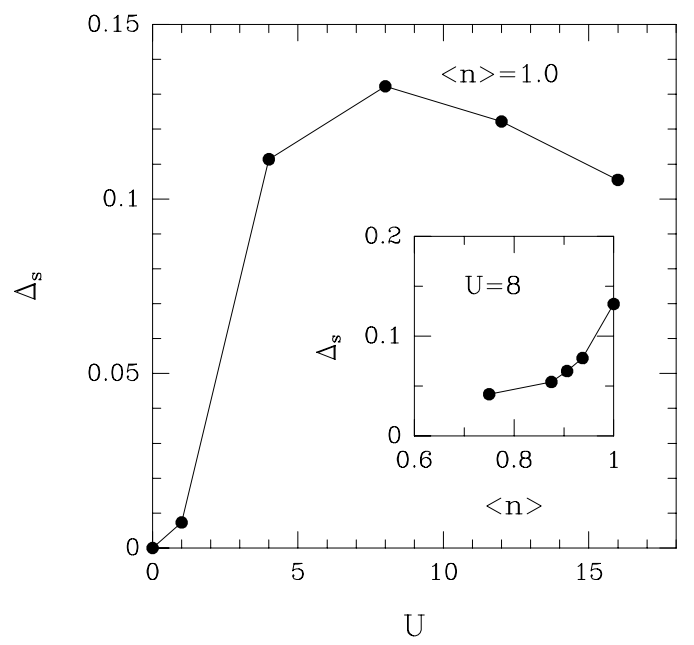

Fig. 29. The spin gap as a function of $U$ for a half filled $2 \times 32$ Hubbard ladder. The inset shows $\Delta_{s}$ as a function of filling $\langle n\rangle$ for $U=8$. Energies are measured in units of $t=1$. (From Noack et al. 342 ) 
Pairs of holes in two leg Hubbard or $t-J$ ladders form bound pairs as can be seen both from the fact that the pair binding energy is positive, and from the fact that positional correlations between holes are indicative of a bound state. The pairs have a predominant $d_{x^{2}-y^{2}}$ symmetry as is revealed by the relative minus sign between the ground state to ground state amplitudes for adding a singlet pair on neighboring sites along and across the legs 192, 341. It seems that the dominance of the $d_{x^{2}-y^{2}}$ channel is universally shared by all models over the entire range of doping that has been studied. (See Section 10.3 for a discussion of this phenomenon in the $2 \times 2$ plaquette.)

The doping dependence of the pair binding energy roughly follows the spin gap in various versions of the two leg ladder as shown in Fig. 30. The correlation function $D(l)$ of the pair field

$$
\Delta_{i}^{\dagger}=\left(c_{i 1 \uparrow}^{\dagger} c_{i 2 \downarrow}^{\dagger}-c_{i 1 \downarrow}^{\dagger} c_{i 2 \uparrow}^{\dagger}\right),
$$

exhibits behavior consistent with a power law decay [192, 341, 343, 344]

$$
D(l)=\left\langle\Delta_{i+l} \Delta_{i}^{\dagger}\right\rangle \sim l^{-\theta} .
$$

There exists less data concerning its doping dependence, but from the relevant studies 192, 341] we can conclude that the pair correlations increase from the undoped system to a maximum at $x \sim 0.0625$ and then decrease when more holes are added to the system.

Both the spin gap and the pairing correlations in doped Hubbard and $t-J$ ladders can be appreciably enhanced by slight generalizations of the models. For example, the exponent $\theta$ in Eq. (128), which depends on the coupling strengths $U / t$ or $J / t$ and the doping level $x$, is also sensitive to the ratio of the hopping amplitudes between neighboring sites on a rung and within a chain $t_{\perp} / t$. By varying this parameter, the exponent $\theta$ can be tuned over the range $0.9 \leq \theta \leq 2.1$. In particular, for $x=0.0625$ and intermediate values of the (repulsive) interaction $5 \leq U / t \leq 15$, it can be made smaller than 1 [192]; see Fig. 14. This is significant since, as we saw in Section 5.1, whenever $\theta<1$ the superconducting susceptibility is the most divergent among the various susceptibilities of the ladder. Adding a nearest neighbor exchange coupling, $J$, to $H_{U}$ also leads to stronger superconducting signatures owing to an increase in the pair mobility and binding energy [346]. The moral here is that details are important as far as they reveal the nonuniversal properties of the Hamiltonians that we study, and indicate relevant directions in model space. It should also imprint on us a sense of humility when attempting to fit real world data with such theoretical results.

We already noted that, in contrast to the two leg ladder, the three leg system does not possess a spin gap at half filling. This situation persists up to hole doping of about $x=1-\langle n\rangle=0.05$, as can be seen in Fig. 31. ${ }^{2}$ However,

${ }^{24}$ The nonvanishing spin gap in this region is presumably a finite size effect; see Fig. 28.
Holes like to d-pair.

Details and their importance

Another lesson in
humility

Odd and want a gap? -Dope! 


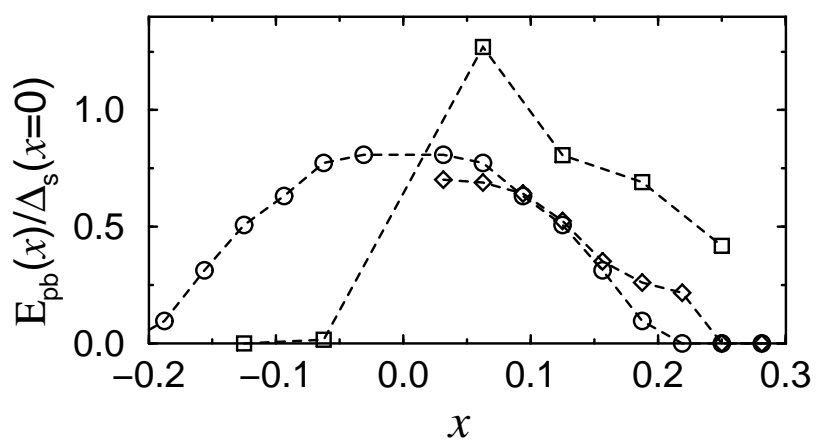

Fig. 30. The ratio of the pair binding energy to the undoped spin gap as a function of hole doping $x=1-\langle n\rangle$. The diamonds are for a $32 \times 2 t-J$ ladder with $J / t=0.3$. The circles are for a one band $32 \times 2$ Hubbard ladder with $U / t=12$. The squares are for a three band Hubbard model of a two leg $\mathrm{Cu}-\mathrm{O}$ ladder, i.e. a ladder made of $\mathrm{Cu}$ sites where nearest neighbor sites are connected by a link containing an $\mathrm{O}$ atom. Here $U_{d} / t_{p d}=8$, where $U_{d}$ is the on-site $\mathrm{Cu}$ Coulomb interaction and $t_{p d}$ is the hopping matrix element between the $\mathrm{O}$ and $\mathrm{Cu}$ sites. The energy difference between the $\mathrm{O}$ and $\mathrm{Cu}$ sites is $\left(\epsilon_{p}-\epsilon_{d}\right) / t_{p d}=2$, and the calculation is done on a $16 \times 2$ ladder. (From Jeckelmann et al. 345)

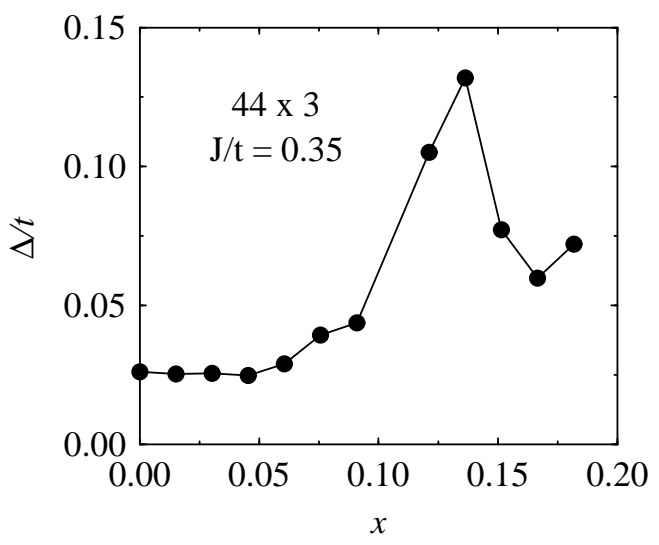

Fig. 31. Spin gap for a $44 \times 3$ ladder with open boundary conditions and $J / t=0.35$ as a function of doping. (From White and Scalapino. 347])

with moderate doping a spin gap is formed which reaches a maximum value at a doping level of $x=0.125$. For the system shown here, with $J / t=0.35$, the gap is only 20 percent smaller than that of the undoped two leg Heisenberg ladder. Upon further doping, the spin gap decreases and possibly vanishes as $x$ gets to be 0.2 or larger. 


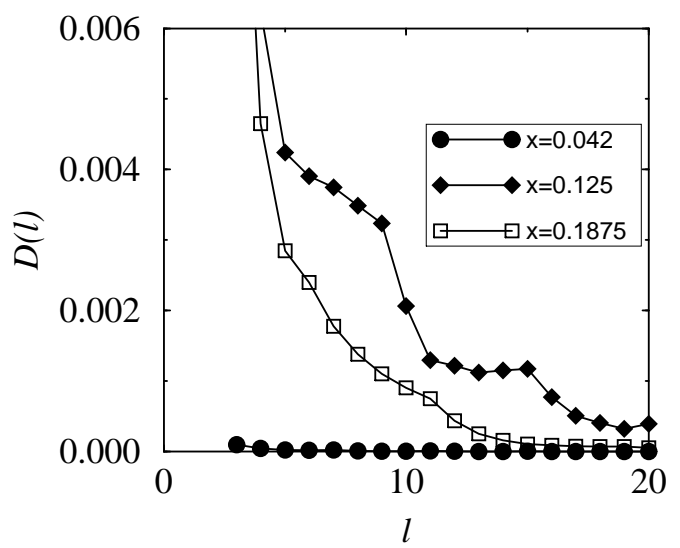

Fig. 32. The $d_{x^{2}-y^{2}}$ pair field correlations $D(l)$ for three different densities, calculated on $32 \times 3(x=0.1875)$ and $48 \times 3(x=0.042, x=0.125)$ open $t-J$ ladders with $J / t=0.35$. (From White and Scalapino. 347)

The establishment of a spin gap is concurrent with the onset of pairing correlations in the system. While two holes introduced into a long, half filled three chain ladder do not bind [314], indications of pairing emerge as soon as the spin gap builds up [347,348]. As an example, Fig. 32 plots the pair field-pair field correlation function of Eq. (128) for various values of the hole doping, defined with

$$
\Delta_{i}^{\dagger}=c_{i, 2 \uparrow}^{\dagger}\left(c_{i+1,2 \downarrow}^{\dagger}+c_{i-1,2 \downarrow}^{\dagger}-c_{i, 1 \downarrow}^{\dagger}-c_{i, 3 \downarrow}^{\dagger}\right)-(\uparrow \leftrightarrow \downarrow)
$$

which creates a $d_{x^{2}-y^{2}}$ pair around the $i$ th site of the middle leg (the leg index runs from 1 to 3 ).25 In the regime of low doping $x \leq 0.05$, the pair field correlations are negligible. However, clear pair field correlations are present at $x=0.125$, where they are comparable to those in a two leg ladder under similar conditions. The pair field correlations are less strong at $x=0.1875$; they follow an approximate power law decay as a function of the distance. [344, 347] (The oscillations in $D(l)$ are produced by the open boundary conditions used in this calculation.) This behavior can be understood from strong coupling bosonization considerations [20] in which the two even modes (with respect to reflection about the center leg) form a spin gapped two leg ladder and for small doping the holes enter the odd mode giving rise to a gapless one dimensional electron gas. As the doping increases, pair hopping between the two subsystems may induce a gap in the gapless channel via the spin gap proximity effect 20].

Increasing the number of legs from three to four leads to behavior similar to that exhibited by the two leg ladder. The system is spin gapped and two

$\overline{25}$ There also exists a small $s$-wave component in the pair field due to the one dimensional nature of the cluster. 


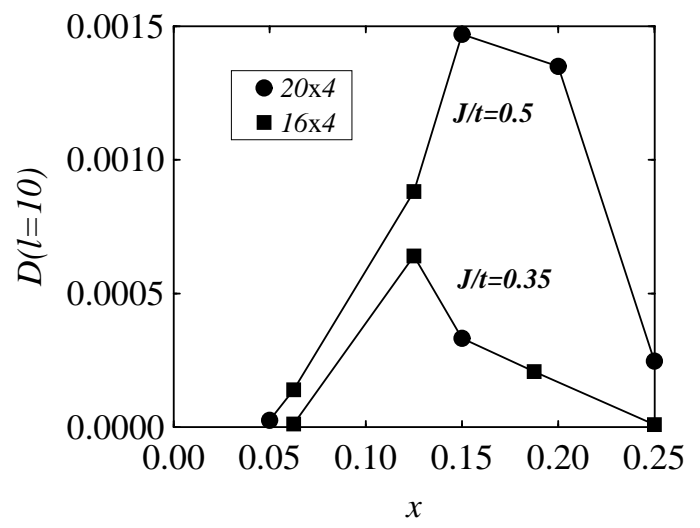

Fig. 33. The $d_{x^{2}-y^{2}}$ pair field correlation $D(l)$ at a separation of $l=10$ rungs as a function of doping $x$, for $20 \times 4$ and $16 \times 4$ open ladders with $J / t=0.35$ and 0.5 . (From White and Scalapino. [349])

holes in a half filled four leg ladder tend to bind. The pair exhibits features common to all pairs in an antiferromagnetic environment, including a $d$-wavelike symmetry [314]. Further similarity with the two leg ladder is seen in the $d$-wave pair field correlations $D(l)$. Fig. 33 shows $D(l=10)$ for a $t-J$ four leg ladder as a function of doping (extended $s$-wave correlations are much smaller in magnitude). The pairing correlations for $J / t=0.5$ increase with doping, reaching a maximum between $x=0.15$ and $x=0.2$, and then decrease.

Four legs are good; two legs are better.
The magnitude of the correlations near the maximum is similar to that of a two leg Hubbard ladder with $U=8 t$ (corresponding to $J \sim 4 t^{2} / U=0.5$ ) with the same doping, but smaller than the maximum in the two leg ladder which occurs at smaller doping 192, 341]. For $J / t=0.35$ the peak is reduced in magnitude and occurs at lower doping. The behavior of $D(l)$ near the maximum is consistent with power law decay for short to moderate distances but seems to fall more rapidly at long distances (perhaps even exponentially. (350)

Lastly, we present in Fig. 34 the response of a few ladder systems to a proximity pairing field

$$
H_{1}=d \sum_{i}\left(c_{i, \uparrow}^{\dagger} c_{i+\hat{y}, \downarrow}^{\dagger}-c_{i, \downarrow}^{\dagger} c_{i+\hat{y}, \uparrow}^{\dagger}+\text { h.c. }\right)
$$

which adds and destroys a singlet electron pair along the ladder. The response is given by the average $d_{x^{2}-y^{2}}$ pair field

$$
\left\langle\Delta_{d}\right\rangle=\frac{1}{N} \sum_{i}\left\langle\Delta_{i}\right\rangle,
$$

with $\Delta_{i}$ defined in Eq. (127). We see that the pair field response tends to decrease somewhat with the width of the system but is overall similar for the 
two, three and four leg ladders. We suspect it gets rapidly smaller for wider ladders.

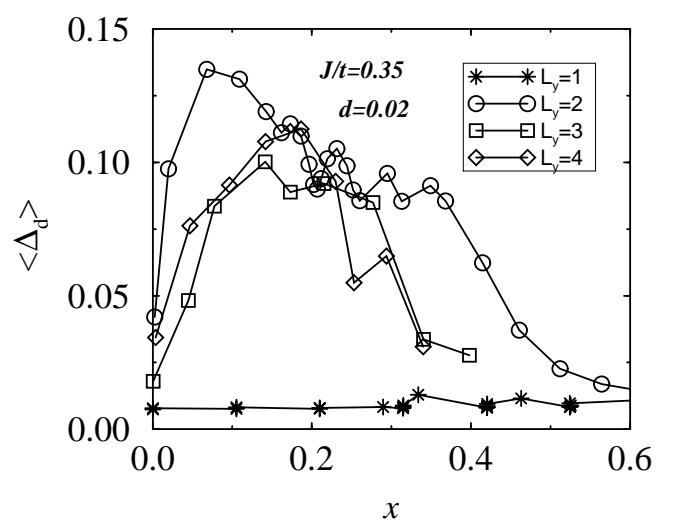

Fig. 34. The $d_{x^{2}-y^{2}}$ pairing response to a proximity pair field operator as a function of doping for a single chain and two, three, and four leg ladders. For the single chain, near neighbor pairing is measured. (From White and Scalapino. 347])

Phase separation and stripe formation in ladders We now address the issue of whether there is any apparent tendency to form charge density and/or spin density wave order in ladder systems, and whether there is a tendency of the doped holes to phase separate. Since incommensurate density wave long range order, like superconducting order, is destroyed by quantum fluctuations in one dimension, we will again be looking primarily at local correlations, rather than actual ordered states. Of course, we have in mind that local correlations and enhanced susceptibilities in a one dimensional context can be interpreted as indications that in two dimensions true superconductivity, stripe order, or phase separation may occur.

Phase Separation: Phase separation was first found in the one dimensional chain [351, 352] and subsequently in the two leg ladder [353 355]. As a rule, the phase separation line has been determined by calculating the coupling $J$ at which the compressibility diverges. (See, however, Ref. 322.) This is in principle an incorrect criterion. The compressibility only diverges at the consolute point. Thermodynamically appropriate criteria for identifying regimes of phase separation from finite size studies include the Maxwell construction (discussed explicitly in Section 12, below), and measurements of the surface tension in the presence of boundary conditions that force phase coexistence. The divergent compressibility is most directly related to the spinodal line, which is not even strictly well defined beyond mean field theory. Thus, while 
in many cases the phase diagrams obtained in this way may be qualitatively correct, they are always subject to some uncertainty.

More recently Rommer, White, and Scalapino [356] have used DMRG methods to extend the study to ladders of up to six legs. Since these calculations are carried out with open boundary conditions, which break the translational symmetry of the system, they have used as their criterion the appearence of an inhomogeneous state with a hole rich region at one edge of the ladder and hole free regions near the other, which is a thermodynamically correct criterion for phase separation. However, where the hole rich phase has relatively low hole density, and in all cases for the six leg ladder, they were forced to use a different criterion which is not thermodynamic in character, but is at least intuitively appealing. From earlier studies (which we discuss below) it appears that the "uniform density" phase, which replaces the phase separated state for $J / t$ less than the critical value for phase separation, is a "striped" state, in which the holes congregate into puddles (identified as stripes) with fixed number of holes, but with the density of stripes determined by the mean hole density on the ladder. With this in mind, Rommer et al. computed the interaction energy between two stripes, and estimated the phase separation boundary as the point at which this interaction turns from repulsive to attractive. The results, summarized in Fig. 35, agree with the thermodynamically determined phase boundary where they can be compared.

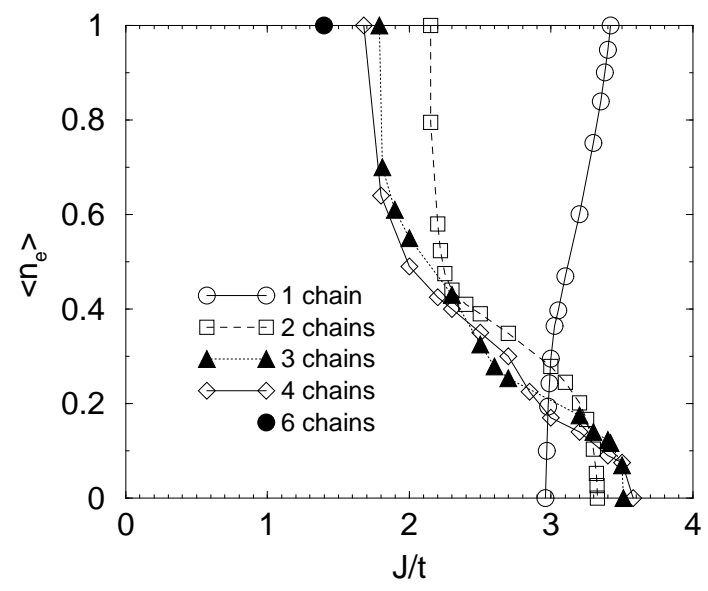

Fig. 35. Boundary to phase separated region in $t-J$ ladders. Open boundary conditions were used in both the leg and rung directions except for the six leg ladder where periodic boundary conditions were imposed along the rung. Phase separation is realized to the right of the curves. $\left\langle n_{e}\right\rangle$ is the total electron density in the system. (From Rommer et al. 356.) 
For large enough values of $J / t$, both the single chain and the ladders are fully phase separated into a Heisenberg phase $\left(\left\langle n_{e}\right\rangle=1\right)$ and an empty phase $\left(\left\langle n_{e}\right\rangle=0\right)$. However, the evolution of this state as $J / t$ is reduced is apparently different for the two cases. For the chain, the Heisenberg phase is destroyed first by holes that diffuse into it; this presumably reflects the fact that hole motion is not significantly frustrated in the single chain system. In the ladders, on the other hand, the empty phase is the one that becomes unstable due to the sublimation of electron pairs from the Heisenberg region. This difference is evident in Fig. 35 where the phase separation boundary occurs first at high electron density in the chain and high hole density in the ladders. It is also clear from looking at this figure that the value of $J / t$ at which phase separation first occurs for small electron densities is hardly sensitive to the width of the ladder. However, as more electrons are added to the system (removing holes), phase separation is realized for smaller values of $J / t$ in wider ladders. Whether this is an indication that phase separation takes place at arbitrarily small $J / t$ for small enough hole densities in the two dimensional system is currently under debate, as we discuss in Section 11.2 .

"Stripes" in ladders: At intermediate values of $J / t$, and not too close to half filling, the doped holes tend to segregate into puddles which straddle the ladders, as is apparent from the spatial modulation of the mean charge density along the ladder. Intuitively, we can think of this state as consisting of an array of stripes with a spacing which is determined by the doped hole density. From this perspective, the total number of doped holes associated with each puddle, $N_{\text {puddle }}=\varrho L$, is interpreted as arising from a stripe with a mean linear density of holes, $\varrho$, times the length of the stripe, $L{ }^{26}$ ( $L$ is also the width of the ladder.) In the thermodynamic limit, long wavelength quantum fluctuations of the stripe array would presumably result in a uniform charge density, but the ladder ends, even in the longest systems studied to date, are a sufficiently strong perturbation that they pin the stripe array [357]. In two and three leg ladders, the observed stripes apparently always have $\varrho=1$. For the four leg ladder, typically $\varrho=1$, but under appropriate circumstances (especially for $x=1 / 8$ ), $\varrho=1 / 2$ stripes are observed. In six and eight leg ladders, the charge density oscillations are particularly strong, and correspond to stripes with $\varrho=2 / 3$ and $1 / 2$, respectively. Various arguments have been presented to identify certain of these stripe arrays as being "vertical" (i.e. preferentially oriented along the rungs of the ladder) or "diagonal" (i.e. preferentially oriented at $45^{\circ}$ to the rung), but these arguments, while intuitively appealing, do not have a rigorous basis.

${ }^{26}$ For instance, on a long, $N$ site, 4 leg ladder with $4 n$ holes, where $n \ll N$, one typically observes $n$ or $2 n$ distinct peaks in the rung-averaged charge density, which is then interpreted as indicating a stripe array with $\varrho=1$ or $\varrho=1 / 2$, respectively.

Ladders phase separate for large enough $J / t$.

Stripes appear at smaller $J / t$. 
We will return to the results on the wider ladders, below, where we discuss attempts to extrapolate these results to two dimensions.

\subsection{Properties of the two dimensional $t-J$ model}

It is a subtle affair to draw conclusions about the properties of the two dimensional Hubbard and $t-J$ models from numerical studies of finite systems. The present numerical capabilities do not generally permit a systematic finite size scaling analysis. As a result, extrapolating results from small clusters with periodic boundary conditions, typically used when utilizing Monte Carlo or Lanczos techniques, or from strips with open boundary conditions as used in DMRG studies, is susceptible to criticism 325, 329]. It comes as no surprise then that several key issues concerning the ground state properties of the two dimensional models are under dispute. In the following we present a brief account of some of the conflicting results and views. However, at least two things do not seem to be in dispute: 1) there is a strong tendency for doped holes in an antiferromagnet to clump in order relieve the frustration of hole motion [358], and 2) where it occurs, hole pairing has a $d_{x^{2}-y^{2}}$ character. Thus, in one way or another, the local correlations that lead to stripe formation and $d$-wave superconductivity are clearly present in $t-J$-like models!

Phase separation and stripe formation There have been relatively few numerical studies of large two dimensional Hubbard model clusters. Monte Carlo simulations on square systems with sizes up to $12 \times 12$ and temperatures down to roughly $t / 8$ have been carried out, typically with $U / t=4$ [330]. A signature of phase separation in the form of a discontinuity in the chemical potential as a function of doping was looked for and not found. No evidence of stripe formation was found, either. Given the limited size and temperature range of these studies, and the absence of results that would permit a Maxwell construction to determine the boundary of phase separation, it is difficult to reach a firm conclusion on the basis of these studies. Certainly at relatively elevated temperatures, holes in the Hubbard model do not show a strong tendency to cluster, but it is difficult to draw conclusions concerning lower temperature, or more subtle tendencies. (Variational "fixed node" studies by Cosentini et al [359] are suggestive of phase separation at small $x$, but more

Everybody agrees on the phase separation boundary for $x \sim 1$. recent studies by Becca et al. 360 reached the opposite conclusion.)

There are many more studies of phase separation in the $t-J$ model. Most of them agree on the behavior in the regime of very low electron density $n_{e}=$ $1-x \ll 1$. The critical $J / t$ value for phase separation at vanishingly small $n_{e}$ was calculated very accurately by Hellberg and Manousakis [361] and was found to be $J / t=3.4367$. However, there are conflicting results for systems close to half filling $\left(n_{e} \sim 1\right)$ and with small $t-J$. This is the most delicate region where high numerical accuracy is hard to obtain. Consequently, there is no agreement on whether the two dimensional $t-J$ model phase separates for all values of $J / t$ at sufficiently low hole doping $x$. 
Emery et al. 362, 363] presented a variational argument (recently extended and substantially improved by Eisenberg et al. [364]) that for $J / t \ll 1$ and for $x$ less than a critical concentration, $x_{c} \sim \sqrt{J / t}$, phase separation occurs between a hole free antiferromagnetic and a metallic ferromagnetic state. Since for large $J / t$ there is clearly phase separation for all $x$, they proposed that for sufficiently small $x$, phase separation is likely to occur for all $J / t$. To test this, they computed the ground state energy by exact diagonalization of $4 \times 4$ doped $t-J$ clusters. If taken at face value and interpreted via a Maxwell construction, these results imply that for any $x<1 / 8$, phase separation occurs at least for all $J / t>0.2$. Hellberg and Manousakis [322, 331] calculated the ground state energy on larger clusters of up to $28 \times 28$ sites using Green function Monte Carlo methods. By implementing a Maxwell construction, they reached the similar conclusion that the $t-J$ model phase separates for all values of $J / t$ in the low hole doping regime.

On the other hand, Putikka et al. [365] studied this problem using a high temperature series expansion extrapolated to $T=0$ and concluded that phase separation only occurs above a line extending from $J / t=3.8$ at zero filling to $J / t=1.2$ at half filling. In other words, they concluded that there is no phase separation for any $x$ so long as $J / t<1.2$. Exact diaganolization results for the compressibility and the binding energy of $n$-hole clusters in systems of up to 26 sites by Poilblanc [366] were interpreted as suggesting that the ground state is phase separated close to half filling only if $J / t>1$. Quantum Monte Carlo simulations of up to 242 sites using stochastic reconfiguration by Calandra et al. [367] have found a phase separation instability for $J / t \sim$ 0.5 at similar doping levels, but no phase separation for $J / t<0.5$, while earlier variational Monte Carlo calculations [368] reported a critical value of $J / t=1.5$. Using Lanczos techniques to calculate the ground state energy on lattices of up to 122 sites, Shin et al. [369, 370] estimate the lower critical value for phase separation as $J / t=0.3-0.5$, a somewhat lower bound than previously found using similar numerical methods 371 . Finally, DMRG calculations on wide ladders with open boundary conditions in one direction by White and Scalapino 320,321 found striped ground states for $J / t=0.35$ and $0<x<0.3$, but no indication of phase separation.

For comparison, we have gathered a few of the results mentioned above in Fig. 36. The scatter of the data at the upper left corner of the $n_{e}-J / t$ plane is a reflection of the near linearity of the the ground state energy as a function of doping in this region [329]. High numerical accuracy is needed in order to establish a true linear behavior which would be indicative of phase separation. While there is currently no definitive answer concerning phase separation at small doping, it seems clear that in this region the two dimensional $t-J$ model is in delicate balance, either in or close to a phase separation instability.

The nature of the ground state for moderately small $J / t$ beyond any phase separated regime is also in dispute. While DMRG calculations on fat
The situation for $x \sim 0$ is murkier, but...

... it seems that the model is either phase separated, or very close to it. 


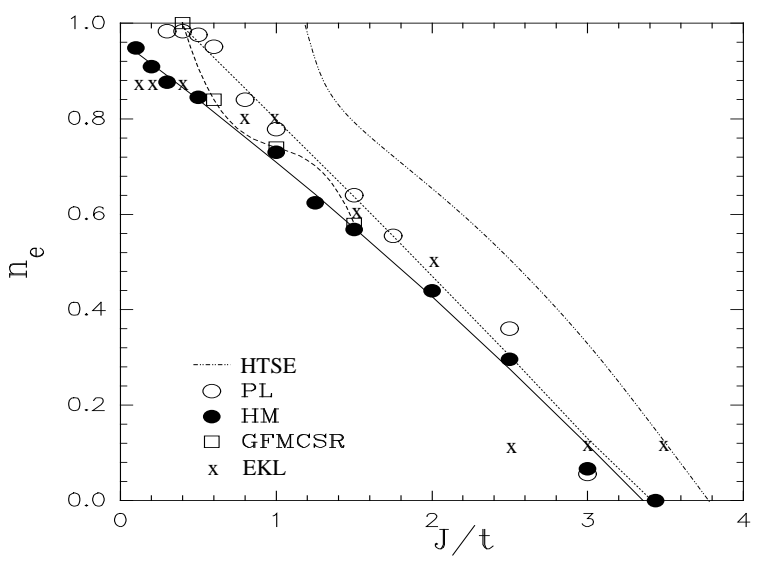

Fig. 36. Phase separation boundary of the two dimensional $t-J$ model according to various numerical studies. The dashed-dotted line represents the high temperature series expansion results by Puttika et al. 365. Also shown are results from calculations using the Power-Lanczos method by Shin et al. [370] (open circles), Greens function Monte Carlo simulations by Hellberg and Manousakis [331] (closed circles) and by Calandra et al. [367] (open squares), and exact diagonalization of $4 \times 4$ clusters by Emery et al. 362] (x's). (Adapted from Shin et al. [370])

Stripes are important low energy configurations of the $t-$ $J$ model.

Typically stripes are quarter-filled antiphase domain walls. ladders 320, 321 find striped ground states for $J / t=0.35$ and $x=1 / 8$, Monte Carlo simulations on a torus [325] exhibit stripes only as excited states. Whether this discrepancy is due to finite size effects or the type of boundary conditions used is still not settled. (The fixed node Monte Carlo studies of Becca et al. 372] likewise conclude that stripes do not occur in the ground state, although they can be induced by the addition of rather modest anisotropy into the $t-J$ model, suggesting that they are at least energetically competitive.) While these conflicting conclusions may be difficult to resolve, it seems inescapable to us that stripes are important low energy configurations of the two dimensional $t-J$ model for small doping and moderatly small $J / t$.

The most reliable results concerning the internal structure of the stripes themselves come from studies of fat $t-J$ ladders, where stripes are certainly a prominent part of the electronic structure. In all studies of ladders, the doped holes aggregate into "stripes" which are oriented either perpendicular or parallel to the extended direction of the ladder, depending on boundary conditions. In many cases the spin correlations in the hole poor regions between stripes locally resemble those in the undoped antiferromagnet but suffer a $\pi$-phase shift across the hole rich stripe. This magnetic structure is vividly apparent in studies for which the low energy orientational fluctuations of the spins are suppressed by the application of staggered magnetic fields on certain boundary sites of the ladders - then, these magnetic correlations 
are directly seen in the expectation values of the spins [373. However, such findings are not universal: in the case of the four leg ladder, with stripes along the ladder rungs, Arrigoni et al. [328] recently showed that in long systems (up to $4 \times 27$ ), these antiphase magnetic correlations are weak or nonexistent, despite strong evidence of charge stripe correlations. Ladder studies have also demonstrated that stripes tend to favor a linear charge density of $\varrho=1 / 2$ along each stripe. 27 Specifically, by applying boundary conditions which force a single stripe to lie along the long axis of the ladder, White et al 321] were able to study the energy of a stripe as a function of $\varrho$. They found an energy which is apparently a smooth function of $\varrho$ (i.e. with no evidence of a nonanalyticity which would lock $\varrho$ to a specific value), but with a pronounced minimum at $\varrho=1 / 2$. Moreover, with boundary conditions favoring stripes perpendicular to the ladder axis, they found that for $x \leq 1 / 8$ stripes tend to form with $\varrho=1 / 2$ so that the spacing between neighboring stripes is approximately $1 / 2 x$, while at larger $x$, a first order transition occurs to "empty domain walls" with $\varrho=1$ and an inter-stripe spacing of $1 / x$. In the region $0.125<x<0.17$ the two types of stripes can coexist.

It is worth noting that the original indications of stripe order came from Hartree-Fock treatments 375 378. Hartree-Fock stripes are primarily spin textures. In comparison to the DMRG results on ladders, they correspond to "empty" ( $\varrho=1)$ antiphase ( $\pi$-phase shifted) domain walls, and so are insulating and overemphasize the spin component of the stripe order, but otherwise capture much of the physics of stripe formation remarkably accurately.

Further insight into the physics that generates the domain walls can be gained by looking more closely at their hole density and spin structures. Both site-centered and bond-centered stripes are observed. They are close in energy and each type can be stabilized by adjusting the boundary conditions [320]. Fig. 37 depicts three site-centered stripes in a $13 \times 8$ system with 12 holes, periodic boundary conditions along the $y$ direction and a $\pi$-shifted staggered magnetic field on the open ends of magnitude $0.1 t$. These stripes are quarterfilled antiphase domain walls. Fig. 38 shows a central section of a $16 \times 8$ cluster containing two bond-centered domain walls. This system is similar to the one considered above except that the magnetic field on the open ends is not $\pi$-shifted. Like their site-centered counterparts, the bond-centered stripes are antiphase domain walls, but with one hole per two domain wall unit cells.

The $\pi$-phase shift in the exchange field across the stripe can probably be traced, in both the bond- and site-centered cases, to a gain in the transverse kinetic energy of the holes. To demonstrate this point consider a pair of holes in a $2 \times 2 t-J$ plaquette, as was done in Section 10.3. One can simulate the effect of the exchange field running on both sides of the plaquette through a mean field $h$ which couples to the spins on the square [373]. For the inphase domain wall such a coupling introduces a perturbation $h\left(S_{1}^{z}-S_{2}^{z}-\right.$

$\overline{27}$ At about the same time, Nayak and Wilczek 374 presented an interesting analytic argument which leads to the same bottom line.

They can be site- or bond-centered.

The topological character of spin stripes can be inferred from local considerations. 


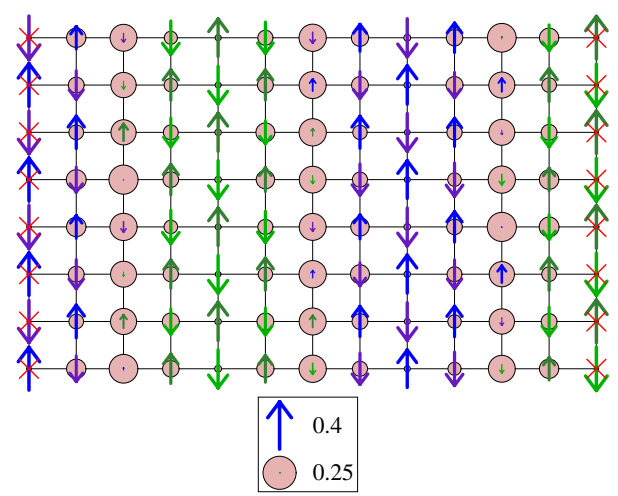

Fig. 37. Hole density and spin moments on a $13 \times 8$ cylinder with 12 holes, $J / t=$ 0.35 , periodic boundary conditions along the $y$ direction and $\pi$-shifted staggered magnetic field of magnitude $0.1 t$ on the open edges. The diameter of the circles is proportional to the hole density $1-\left\langle n_{i}\right\rangle$ and the length of the arrows is proportional to $\left\langle S_{i}^{z}\right\rangle$. (From White and Scalapino. 373 )

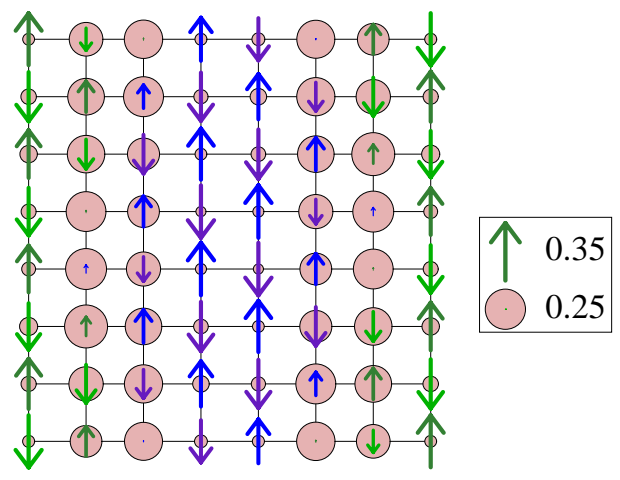

Fig. 38. Hole density and spin moments on a central section of a $16 \times 8$ cylinder with 16 holes, $J / t=0.35$, with periodic boundary conditions along the $y$ direction and staggered magnetic field of magnitude $0.1 t$ on the open edges. The notation is similar to Fig. 37. (From White and Scalapino. 373.)

$\left.S_{3}^{z}+S_{4}^{z}\right)$ which, to lowest order in $h$, lowers the ground state energy by $-h^{2} / \sqrt{J^{2}+32 t^{2}}$. For the $\pi$-shifted stripe the perturbation is $h\left(S_{1}^{z}+S_{2}^{z}-\right.$ $\left.S_{3}^{z}-S_{4}^{z}\right)$ with a gain of $-4 h^{2} / \sqrt{J^{2}+32 t^{2}}$ in energy, thereby being more advantageous for the pair. Indeed, this physics has been confirmed by several serious studies, which combine analyatic and numerical work, by Zachar [379], Liu and Fradkin [380], and Chernyshev et al. [381] These studies indicate that there is a transition from a tendency for in-phase magnetic order across 
a stripe for small $\varrho$, when the direct magnetic interactions are dominant, to antiphase magnetic order for $\varrho>0.3$, when the transverse hole kinetic energy is dominant.

Superconductivity and stripes There is no unambiguous evidence for superconductivity in the Hubbard model. The original finite temperature Monte Carlo simulations on small periodic clusters with $U / t=4$ and $x=0.15$ [330, 382] found only short range pair-pair correlations. The same conclusion was reached by a later zero temperature constrained path Monte Carlo calculation 383 .

There are conflicting results concerning the question of superconductivity in the $t-J$ model.

In the unphysical region of large $J / t$, solid conclusions can be reached: Emery et al 362 showed that proximate to the phase separation boundary at $J / t \leq 3.8$, the hole rich phase (which is actually a dilute electron phase with $x \sim 1$ ) has an $s$-wave superconducting ground state. This result was confirmed and extended by Hellberg and Manousakis [322], who further argued that in the dilute electron limit, $x \rightarrow 1^{-}$, there is a transition from an $s$-wave state for $2<J / t<3.5$ to a $p$-wave superconducting state for $J / t<2$, possibly with a $d$-wave state at intermediate $J / t$. Early Lanczos calculations were carried out by Dagotto and Riera [330, 384, 385, in which various quantitites, such as the pair field correlation function and the superfluid density, were computed to search for signs of superconductivity in $4 \times 4 t-J$ clusters. In agreement with the analytic results, these studies gave strong evidence of superconductivity for large $J / t$. Interestingly, the strongest signatures of superconductivity were found for $J / t=3$ and $x=0.5$ and decayed rapidly for larger $J / t$. This was interpreted as due to a transition into the phase separation region. (Note, however, that all the studies summarized in Fig. 36 suggest that $x=0.5$ is already inside the region that, in the thermodynamic limit, would be unstable to phase separation.)

More recent Monte Carlo simulations by Sorella et al. [386, 387] showed evidence for long range superconducting order in $J / t=0.4$ clusters of up to 242 sites with periodic boundary conditions and for a range of $x>0.1$, as shown in Fig. 39. No signs of static stripes have been found in the parameter region that was investigated in these studies. A slight tendency towards incommensurability appears in the spin structure factor at (and sometimes above) optimal doping, suggesting perhaps very weak dynamical stripe correlations. This finding is in sharp contrast to DMRG 320,321] and other [388. calculations that find striped ground states for the same parameters.

Notwithstanding this controversy, these results seem to add to the general consensus that static stripe order and superconductivity compete. This is not to say that stripes and superconductivity cannot coexist. As we saw, evidence for both stripes and pairing have been found in three and four leg $t-J$ ladders 347, 349. In fact pairing is enhanced in both of these systems when

There is no evidence for superconductivity in the Hubbard model.

There is conflicting evidence for superconductivity in the $t-J$ model.

Static stripes hamper superconductivity, but dynamic stripes may enhance it. 


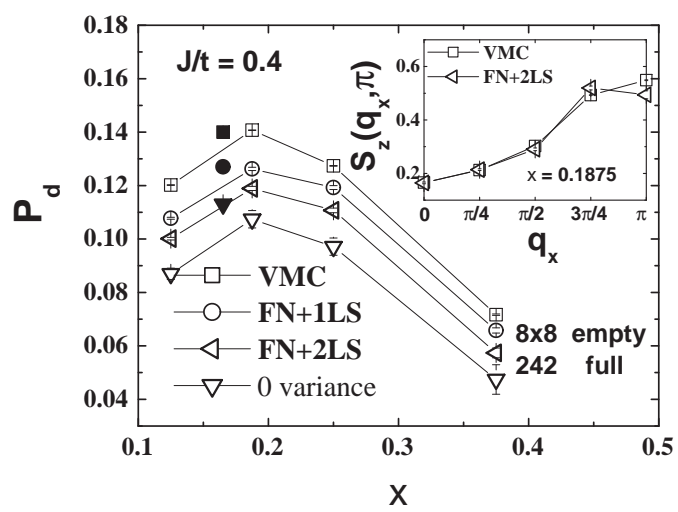

Fig. 39. The superconducting order parameter $P_{d}=2 \lim _{l \rightarrow \infty} \sqrt{D(l)}$ calculated for the largest distance on a $8 \times 8, J / t=0.4$ cluster as function of hole doping $x$. Results for $x=0.17$ on a 242 site cluster are also shown. The different sets of data correspond to various Monte Carlo techniques. The inset shows the spin structure factor at $x=0.1875$. (From Sorella et al. 387)

stripes are formed compared to the unstriped states found at small doping levels. Because of the open boundary conditions that were used in these studies the stripes were open ended and more dynamic. Imposing periodic boundary conditions in wider ladders (and also the four leg ladder) results in stripes that wrap around the periodic direction. These stripes appear to be more static, and pairing correlations are suppressed. A similar behavior is observed when the stripes are pinned by external potentials.

Further evidence for the delicate interplay between stripes and pairing comes from studies of the $t-t^{\prime}-J$ model in which a diagonal, single particle, next nearest neighbor hopping $t^{\prime}$ is added to the basic $t-J$ model [324, 388]. Stripes destabilize for either sign of $t^{\prime}$. This is probably due to the enhanced mobility of the holes that can now hop on the same sublattice without interfering with the antiferromagnetic background. Pairing is suppressed for $t^{\prime}<0$ and enhanced for $t^{\prime}>0 .{ }^{28}$ It is not clear whether the complete elimination of stripes or only a slight destabilization is more favorable to pairing correlations. Fig. 40 suggests that optimal pairing occurs in between the strongly modulated ladder and the homogeneous system.

Finally, allowing for extra hopping terms in the Hamiltonian is not the only way tip the balance between static charge order and superconductivity. So far we have not mentioned the effects of long range Coulomb interactions on the properties of Hubbard related systems. This is not a coincidence since the treatment of such interactions in any standard numerical method is dif-

$\overline{28}$ This is surprising since $T_{c}$ is generally higher for hole doped cuprates (believed to have $t^{\prime}<0$ ) than it is for electron doped cuprates (which have $t^{\prime}>0$ ). 

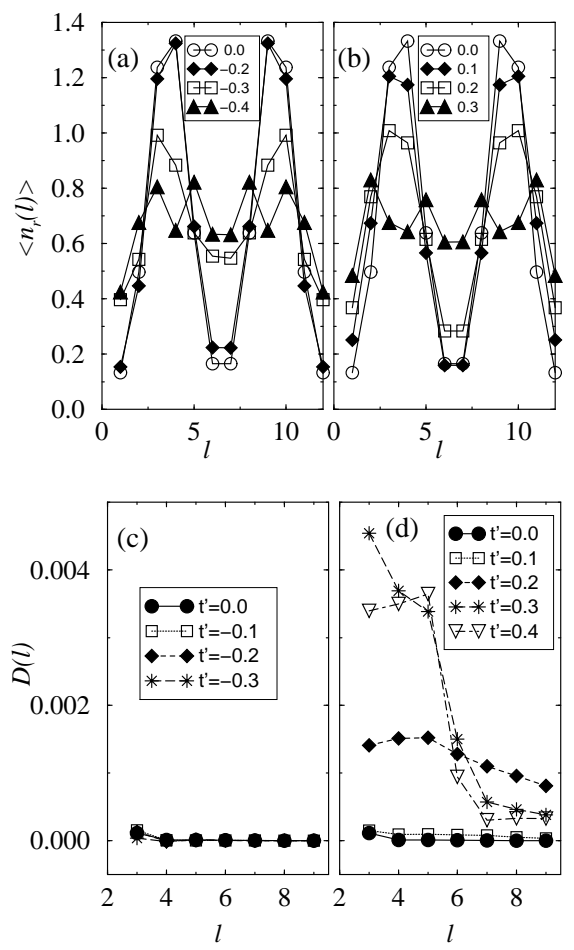

Fig. 40. Hole density per rung for a $12 \times 6$ ladder with periodic boundary conditions along the rungs, 8 holes, $J / t=0.35$ and a) $t^{\prime} \leq 0$ and b) $t^{\prime} \geq 0$. c) and d) depict the $d$-wave pairing correlations for the same systems. (From White and Scalapino. 324])

ficult. Nevertheless, a recent DMRG study of four leg ladders with open and periodic boundary conditions which takes into account the Coulomb potential in a self-consistent Hartree way [328], gives interesting results. It suggests that the inclusion of Coulomb interactions suppresses the charge modulations associated with stripes while enhancing the long range superconducting pairing correlations. At the same time the local superconducting pairing is not suppressed. Taken together, these facts support the notion that enhanced correlations come from long range phase ordering between stripes with wellestablished pairing. This enhanced phase stiffness is presumably due to pair tunnelling between stripes produced by increased stripe fluctuations.

\section{Doped Antiferromagnets}

The undoped state of the cuprate superconductors is a strongly insulating antiferromagnet. It is now widely believed that the existence of such a parent correlated insulator is an essential feature of high temperature supercon- 
ductivity, as was emphasized in some of the earliest studies of this problem [5, 120]. However, the doped antiferromagnet is a complicated theoretical problem - to even cursorily review what is known about it would more than double the size of this document. In this section we very briefly discuss the aspects of this problem which we consider most germane to the cuprates, and in particular to the physics of stripes. More extensive reviews of the subject can be found in $[6,15,358,389$.

\subsection{Frustration of the motion of dilute holes in an antiferromagnet}

The most important local interactions in a doped antiferromagnet are well represented by the large $\mathrm{U}$ Hubbard model, the $t-J$ model, and their various relatives. To be concrete, we will focus on the $t-J-V$ model [363] (a slight generalization of the $t-J$ model, Eq. (126), to which it reduces for for $V=-J / 4$.)

$$
H=-t \sum_{<i, j>, \sigma}\left\{c_{i, \sigma}^{\dagger} c_{j, \sigma}+\text { h.c. }\right\}+\sum_{<i, j>}\left\{J \boldsymbol{S}_{i} \cdot \boldsymbol{S}_{j}+V n_{i} n_{j}\right\}
$$

where $\boldsymbol{S}_{i}=\sum_{\sigma, \sigma^{\prime}} c_{i, \sigma}^{\dagger} \boldsymbol{\sigma}_{\sigma, \sigma^{\prime}} c_{i, \sigma^{\prime}}$ is the spin of an electron on site $i$. Here $\boldsymbol{\sigma}$ are the Pauli matrices and $\langle i, j\rangle$ signifies nearest neighbor sites on a hypercubic lattice in $d$ dimensions. There is a constraint of no double occupancy on any site,

$$
n_{i}=\Sigma_{\sigma} c_{i, \sigma}^{\dagger} c_{i, \sigma}=0,1 .
$$

The concentration of doped holes, $x$, is taken to be much smaller than 1 , and is defined as

$$
x=N^{-1} \sum_{j} n_{j},
$$

where $N$ is the number of sites.

The essential feature of this model is that it embodies a strong, short range repulsion between electrons, manifest in the constraint of no double occupancy. The exchange integral $J$ arises through virtual processes wherein the intermediate state has a doubly occupied site, producing an antiferromagnetic coupling. Doping is assumed to remove electrons thereby producing a "hole" or missing spin which is mobile because neighboring electrons can hop into its place with amplitude $t$.

Like a good game, the rules are simple: antialign adjacent spins, and let holes hop. And like any good game, the winning strategy is complex. The ground state of this model must simultaneously minimize the zero point kinetic energy of the doped holes and the exchange energy, but the two terms

For $t>J>x t$, the problem is highly frustrated. compete. The spatially confined wavefunction of a localized hole has a high kinetic energy; the $t$ term accounts for the tendency of a doped hole to delocalize by hopping from site to site. However, as holes move through an 
antiferromagnet they scramble the spins: each time a hole hops from one site to its nearest neighbor, a spin is also moved one register in the lattice, onto the wrong sublattice. So it is impossible to minimize both energies simultaneously in $d>1$. Moreover, in the physically relevant range of parameters, $t>J>t x$, neither energy is dominant. On the one hand, because $t>J$, one cannot simply perturb about the $t=0$ state which minimizes the exchange energy. On the other hand, because $J>t x$ one cannot simply perturb about the ground state of the kinetic energy.

A number of strategies, usually involving further generalizations of the model, have been applied to the study of this problem, including: large $n$ 390], large $S$ [391, 392], large $d$ 393], small $t / J$ [362], large $t / J$ [362, 394, 395], and various numerical studies of finite size clusters. (Some of the latter are reviewed in Section 11.) For pedagogic purposes, we will frame aspects of the ensuing discussion in terms of the large $d$ behavior of the model since it is tractable, and involves no additional theoretical technology, but similar conclusions can be drawn from a study of any of the analytically tractable limits listed above ${ }^{29}$. One common feature ${ }^{30}$ of these solutions is a tendency of the doped holes to phase separate at small $x$. The reason for this is intuitive: in a phase separated state, the holes are expelled from the pure antiferromagnetic fraction of the system, where the exchange energy is minimized and the hole kinetic energy is not an issue, while in the hole rich regions, the kinetic energy of the holes is minimized, and the exchange energy can be neglected to zeroth order since $J<t x_{\text {rich }}$, where $x_{\text {rich }}$ is the concentration of doped holes in the hole rich regions.

We employ the following large dimension strategy. We take as the unperturbed Hamiltonian the Ising piece of the interaction:

$$
H_{o}=\sum_{<i, j>}\left\{J_{z} S_{i}^{z} S_{j}^{z}+V n_{i} n_{j}\right\},
$$

and treat as perturbations the XY piece of the interaction and the hopping:

$$
\begin{aligned}
& H_{1}=\frac{J_{\perp}}{2} \sum_{<i, j>}\left\{S_{i}^{+} S_{j}^{-}+\text {h.c. }\right\}, \\
& H_{2}=-t \sum_{<i, j>, \sigma}\left\{c_{i, \sigma}^{\dagger} c_{j, \sigma}+\text { h.c. }\right\} .
\end{aligned}
$$

Expansions derived in powers of $J_{\perp} / J_{z}$ and $t / J_{z}$ can be reorganized in powers of $1 / d$, 393] at which point we will again set $J_{\perp}=J_{z} \equiv J$ as in the original model (Eq. (132)), and allow the ratio $t / J$ to assume physical values.

${ }^{29}$ In some ways, the large $S$ limit is the most physically transparent of all these approaches - see Ref. 392 for further discussion.

${ }^{30}$ It is still controversial whether or not phase separation is universal in $d=2$ and 3 at small enough $x$ - see Refs. 322, 329, 358, 369, 396 398. 
The motion of one hole in an antiferromagnet is frustrated.
One hole in an antiferromagnet It is universally recognized that a key principle governing the physics of doped antiferromagnets is that the motion of a single hole is highly frustrated. To illustrate this point, it is convenient to examine it from the perspective of a large dimension treatment in which the motion of one hole in an antiferromagnet is seen to be frustrated by a "string" left in its wake (see Fig. 41), which costs an energy of order $(d-1) J$ times the length of the string. The unperturbed ground state of one hole on, say, the "black" sublattice, is $N / 2$-fold degenerate (equal to the number of black sublattice sites), once a direction for the Néel order is chosen (the other $N / 2$ degenerate ground states describing a hole on the "red" sublattice form a disjoint Hilbert space under the operation of $H_{1}$ and $H_{2}$ ). These ground states are only connected in degenerate perturbation theory of third or higher order, via, e.g. two operations of $H_{2}$ and one of $H_{1}$. They are connected

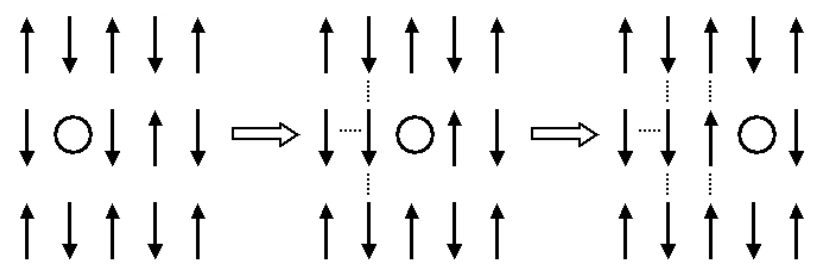

Fig. 41. Frustration of one hole's motion in an antiferromagnet. As the hole hops, it leaves behind a string of frustrated bonds designated here by dashed lines.

in perturbation theory of sixth or higher order by operations solely of the hopping term $H_{1}$ via the Trugman [399] terms, in which a hole traces any closed, nonintersecting path two steps less than two full circuits; see Fig. 42 for an example (such paths become important when $J \ll t$ ). In this manner a hole can "eat its own string". Owing to such processes a hole can propagate through an antiferromagnet. However, the high order in the perturbation series and the energetic barriers involved render the effective hopping matrix elements significantly smaller than their unperturbed values.

Two holes in an antiferromagnet In early work on high temperature superconductivity, it was often claimed that, whereas the motion of a single hole is inhibited by antiferromagnetic order, pair motion appears to be entirely unfrustrated. It was even suggested [19] that this might be the basis of a novel, kinetic energy driven mechanism of pairing - perhaps the first such suggestion. However, a flaw with this argument was revealed in the work of Trugman 399], who showed that this mode of propagation of the hole pair is frustrated by a quantum effect which originates from the fermionic character of the background spins. While Trugman's original argument was based on a careful analysis of numerical studies in $d=2$, the same essential effect 

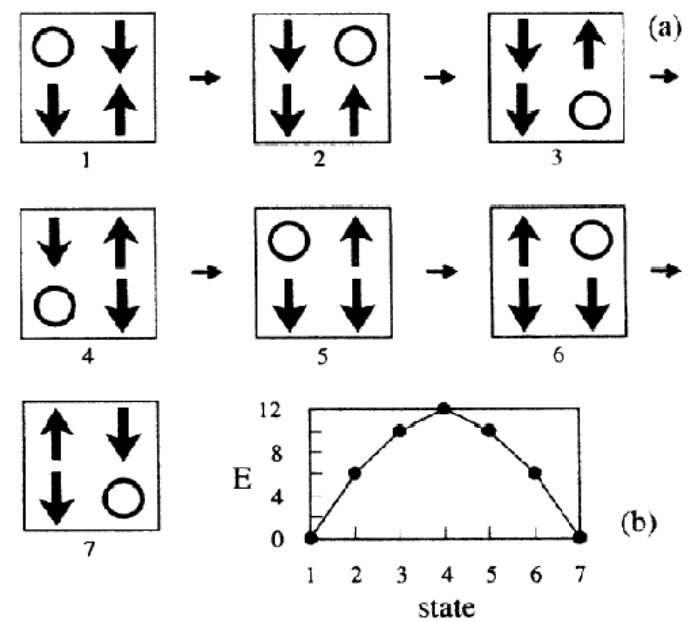

(b)

Fig. 42. Trugman terms. (a) A hole moving one and a half times around a plaquette translates a degenerate ground state without leaving a frustrated string of spins behind. (b) The energy of the intermediate states in units of $J$. The hole has to tunnel through this barrier as it moves. From Ref. 399.

can be seen analytically in the context of a large $d$ expansion. The effective Hamiltonian of two holes can be written as follows [393]:

$$
H_{2}^{e f f}=U^{e f f} \sum_{<i, j>} c_{i}^{\dagger} c_{j}^{\dagger} c_{j} c_{i}-T^{e f f} \sum_{<i, j, k>} c_{j}^{\dagger} c_{i}^{\dagger} c_{j} c_{k}+\mathcal{O}\left(1 / d^{2}\right)
$$

where $\langle i, j, k\rangle$ signifies a set of sites such that $i$ and $k$ are both nearest neighbors of $j$, and the $c_{i}^{\dagger}$ creates a hole at site $i$. To lowest order in $(1 / d)$, $U^{e f f}=V-J / 4$ and $T^{e f f}=t^{2} / J d$. For states with the two holes as nearest neighbors, $H_{2}^{\text {eff }}$ can be block diagonalized by Fourier transform, yielding $d$ bands of eigenstates labeled by a band index and a Bloch wavevector $\boldsymbol{k}$. The result is that $d-1$ of these bands have energy $U^{e f f}$ and do not disperse. The remaining band has energy $U^{e f f}+4 T^{e f f} \sum_{a=1}^{d} \sin ^{2}\left(k_{a} / 2\right)$, where $k_{a}$ is the component of $\boldsymbol{k}$ along $a$. This final band, which feels the effects of pair propagation, has the largest energy. This counterintuitive result follows from the fermionic nature of the background spins. A similar calculation for bosons would differ by a minus sign: in that case, the final band has energy $U^{e f f}-4 T^{e f f} \sum_{a=1}^{d} \sin ^{2}\left(k_{a} / 2\right)$, which is much closer to what one might have expected.11 The interference effect for the fermionic problem is illustrated in Fig. 43. Different paths that carry the system from one hole pair configuration to another generally interfere with each other, and when two such paths differ by the exchange of two electrons, they interfere destructively in the fermionic

31 This corrects similar expressions in Ref. 393.

Two holes are no less frustrated. 


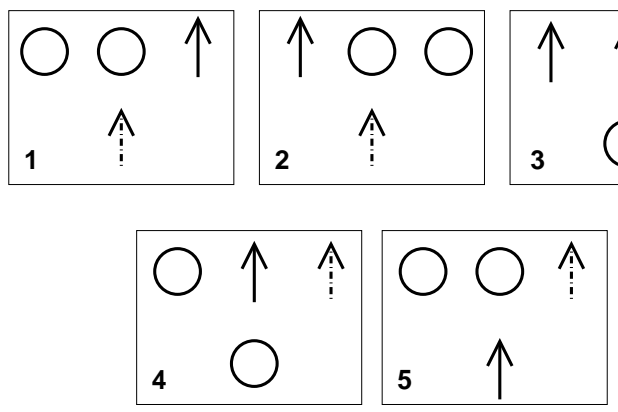

Fig. 43. Frustration of a hole pair's motion in an antiferromagnet. The figure shows a sequence of snapshots in a process that takes a pair of holes back to their original position, but with a pair of spins switched. The sequence is as follows: 1) Initial two hole state. 2) A spin has moved two sites to the left. 3) The other spin has moved one site up. 4) A hole has moved two sites to the left. 5) A hole has moved up. Due to the fermionic nature of the spins, the above process leads to an increase in the pair energy, so that pair propagation is not an effective mechanism of pair binding.

case and constructively in the bosonic. It follows from this argument that pair motion, too, is frustrated - it actually results in an effective kinetic repulsion between holes, rather than in pair binding ${ }^{32}$.

Many holes: phase separation In large $d$, the frustration of the kinetic energy of doped holes in an antiferromagnet leads to a miscibility gap [393]. Perhaps this should not be surprising, since phase separation is the generic fate of mixtures at low temperatures. At any finite temperature, two-phase coexistence occurs whenever the chemical potentials of the two phases are equal. In the present case, one of the phases, the undoped antiferromagnet, is incompressible, which means that at $T=0$ its chemical potential lies at an indeterminate point within the Mott gap. Under these circumstances, phase coexistence is instead established by considering the total energy of the system:

$$
\begin{aligned}
E_{t o t} & =N_{A F} e_{A F}+N_{h} e_{h} \\
& =N e_{A F}+N_{h}\left(e_{h}-e_{A F}\right),
\end{aligned}
$$

$\overline{32}$ It is apparent that second neighbor hopping terms, $t^{\prime}$, produce less frustration of the single particle motion, and "pair hopping" terms, which arise naturally in the $t / U$ expansion of the Hubbard model, lead to unfrustrated pair motion [156]. However, $t^{\prime}$ is generally substantially smaller than $t$, and if pair hopping is derived from the Hubbard model, it is of order $J$, and hence relatively small. 
where $N_{A F}$ and $N_{h}$ are the number of sites occupied by the undoped antiferromagnet and by the hole rich phase, respectively; $N=N_{A F}+N_{h} ; e_{A F}$ is the energy per site of the antiferromagnet and $e_{h}$ is the energy per site of the hole rich phase, in which the concentration of doped holes is $x_{\text {rich }}=x\left(N / N_{h}\right) \geq x$. If $E_{\text {tot }}$ has a minimum with respect to $N_{h}$ at a value $N_{h}<N$, there is phase coexistence. This minimization leads to the equation

$$
\mu=\frac{e_{A F}-e_{h}(\mu)}{1-n(\mu)}
$$

where $\mu$ is the chemical potential of the hole rich phase, and $n=1-x_{c}$ is the electron density in the hole rich phase.

As we shall see, in the limit of large dimension, $n(\mu)$ (and hence $e_{h}$ as well) is either 0 or exponentially small, so Eq. (140) reduces to

$$
\mu \approx e_{A F}
$$

We can see already how phase separation can transpire. As the electron density is raised from zero (i.e. starting from $x=1$ and lowering $x$ ), the chemical potential of the electron gas increases. Once $\mu$ reaches $e_{A F}$, the added electrons must go into the antiferromagnetic phase, and the density of the electron gas stops increasing. We can employ a small $k$ expansion of the electronic dispersion, $\epsilon(\boldsymbol{k})=-2 t d+t k^{2}+\ldots$, to determine that $\mu \approx-2 t d+t k_{F}^{2}$. Thus if $e_{A F}<-2 t d$, the electron gas is completely unstable, and there is phase separation into the pure antiferromagnet, and an insulating hole rich phase with $n=0$. In this case, $x_{c}=1$. Otherwise, the density of the electron gas is

$$
n=\frac{2 A_{d}}{d}\left(\frac{k_{F}}{2 \pi}\right)^{d}=\frac{2 A_{d}}{d}\left(\frac{\sqrt{(\mu+2 t d) / t}}{2 \pi}\right)^{d} .
$$

Here $A_{d}$ is the hypersurface area of a $d$ dimensional unit sphere. In large $d$, the energy per site of the pure antiferromagnet approaches that of the classical Néel state:

$$
e_{A F}=-d\left(\frac{J}{4}-V\right)\left[1+\mathcal{O}\left(1 / d^{2}\right)\right]
$$

From this, it follows that the hole rich phase is insulating (i.e. it has no electrons) if $J-4 V>8 t$ and it is metallic $\left(x_{c}<1\right)$ if $J-4 V<8 t$. However, even when the hole rich phase is metallic, its electron density is exponentially small (as promised):

$$
n=1-x_{c}=\frac{2}{\sqrt{\pi d}}\left[\frac{e}{\pi}\left(1-\left[\frac{J-4 V}{8 t}\right]\right)\right]^{d / 2}[1+\mathcal{O}(1 / d)],
$$

where we have used the asymptotic large $d$ expression 393$] A_{d} \approx \sqrt{\frac{d}{\pi}}\left(\frac{2 \pi e}{d}\right)^{d / 2}$. As illustrated in Fig. 44 in large $d$, so long as $0<x<x_{c}$, the ground state of

Phase separation occurs below a critical concentration of doped holes. 


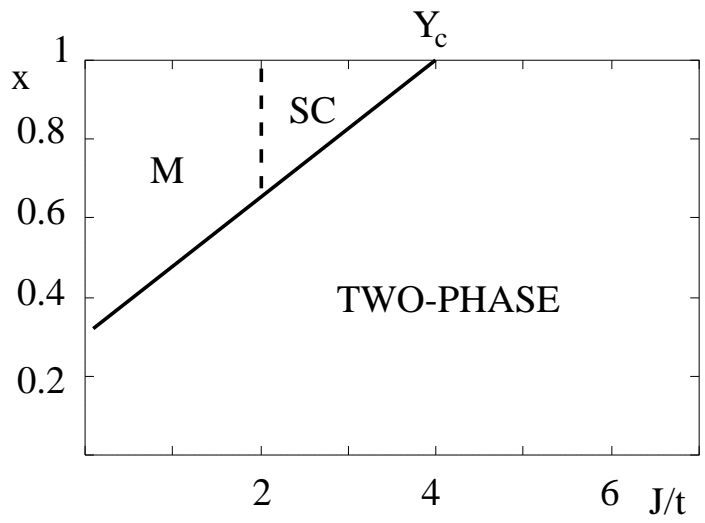

Fig. 44. Phase diagram of the $t-J$ model deduced from large the $d$ expansion. In the figure, we have set $d=2$. "Two-phase" labels the region in which phase separation occurs between the pure antiferromagnet and a hole rich phase, "SC" labels a region of $s$-wave superconductivity, and "M" labels a region of metallic behavior. At parametrically small $J / t \propto 1 / \sqrt{d}$, a ferromagnetic phase intervenes at small doping. From Ref. 393.

the $t-J-V$ model is phase separated, with an undoped antiferromagnetic region and a hole rich region which, if $8 t>J-4 V$, is a Fermi liquid of dilute electrons, or if $8 t<J-4 V$, is an insulator. (Under these same circumstance, if $x_{c}<x<1$, the ground state is a uniform, Fermi liquid meta ${ }^{33}$.)

In the low dimensions of physical interest, such as $d=2$ and $d=3$, the quantitative accuracy of a large dimension expansion is certainly suspect. Nonetheless, we expect the qualitative physics of $d=2$ and $d=3$ to be captured in a large dimension treatment, since the lower critical dimension of most long range $T=0$ ordered states is $d=1$. For comparison, in Fig. 45 we reproduce the phase diagram of the $2 \mathrm{D} t-J$ model which was proposed by Hellberg and Manousakis [322] on the basis of Monte Carlo studies of systems with up to 60 electrons. There is clearly substantial similarity between this and the large $D$ result in Fig. 44 .

In one sense phase separation certainly can be thought of as a strong attractive interaction between holes, although in reality the mechanism is more properly regarded as the ejection of holes from the antiferromagnet. 34 The characteristic energy scale of this interaction is set by magnetic energies, so one expects to see phase separation only at temperatures that are small compared to the antiferromagnetic exchange energy $J$.

$\overline{33}$ This statement neglects a possible subtlety due to the Kohn-Luttinger theorem. ${ }^{34}$ Like salt crystallizing from a solution of salt water, the spin crystal is pure. 


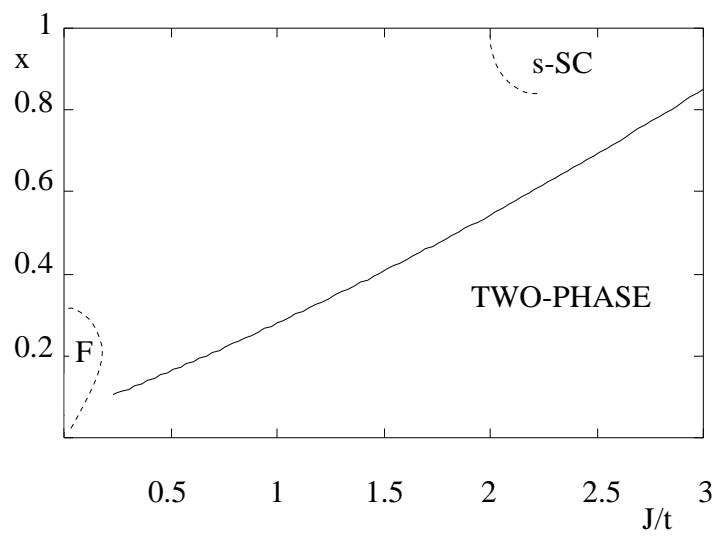

Fig. 45. Phase diagram of the $t-J$ model in two dimensions at zero temperature, deduced from numerical studies with up to 60 electrons. "Two-phase" labels the region of phase separation, "s-SC" labels a region of $s$-wave superconductivity, and "F" labels a region of ferromagnetism. This figure is abstracted from Hellberg and Manousakis [322].

\subsection{Coulomb frustrated phase separation and stripes}

Were holes neutral, phase separation would be a physically reasonable solution to the problem of frustrated hole motion in an antiferromagnet. But there is another competition if the holes carry charge. In this case, full phase separation is impossible because of the infinite Coulomb energy density it would entail. Thus, there is a second competition between the short range tendency to phase separation embodied in the $t-J$ model, and the long range piece of the Coulomb interaction. The compromise solution to this second level of frustration results in an emergent length scale 400 - a crossover between phase separation on short length scales, and the required homogeneity on long length scales. Depending upon microscopic details, many solutions are possible 401] which are inhomogeneous on intermediate length scales, such as checkerboard patterns, stripes, bubbles, or others.

Of these, the stripe solution is remarkably stable in simple models 362 , 393,402], and moreover is widely observed in the cuprates [6]. A stripe state is a unidirectional density wave state - we think of such a state, at an intuitive level, as consisting of alternating strips of hole rich and hole poor phase. A fully ordered stripe phase has charge density wave and spin density wave order interleaved.

Certain aspects of stripe states can be made precise on the basis of long distance considerations. If we consider the Landau theory [45] of coupled order parameters for a spin density wave $\boldsymbol{S}$ with ordering vector $\boldsymbol{k}$ and a charge density wave $\rho$ with ordering vector $\boldsymbol{q}$, then if $2 \boldsymbol{k} \equiv \boldsymbol{q}$ (where $\equiv$, in this case, means equal modulo a reciprocal lattice vector), then there is a

Stripes are a unidirectional density wave. 
cubic term in the Landau free energy allowed by symmetry,

$$
F_{\text {coupling }}=\gamma_{\text {stripe }}\left[\rho_{-\boldsymbol{q}} \boldsymbol{S}_{\boldsymbol{k}} \cdot \boldsymbol{S}_{\boldsymbol{k}}+\text { C.C. }\right] \text {. }
$$

There are two important consequences of this term. Firstly, the system can lower its energy by locking the ordering vectors of the spin and charge density wave components of the order, such that the period of the spin order is twice that of the charge order. At order parameter level, is the origin of the antiphase character of the stripe order ${ }^{35}$. Secondly, because this term is linear in $\rho$, it means that if there is spin order, $\left\langle\boldsymbol{S}_{\boldsymbol{k}}>\neq 0\right.$, there must necessarily be charge order, $\left\langle\rho_{2 k}\right\rangle \neq 0$, although the converse is not true.

The Landau theory also allows us to distinguish three macroscopically distinct scenarios for the onset of stripe order. If charge order onsets at a higher critical temperature, and spin order either does not occur, or onsets at a lower critical temperature, the stripe order can be called "charge driven." If spin and charge order onset at the same critical temperature, but the charge order is parasitic, in the sense that $\left\langle\rho_{2 \boldsymbol{k}}>\sim<\boldsymbol{S}_{\boldsymbol{k}}\right\rangle^{2}$, the stripe order is "spin driven." Finally, if charge and spin order onset simultaneously by a first order transition, the stripe order is driven by the symbiosis between charge and spin order. This is discussed in more detail in Ref. 45 .

The antiphase nature of the stripes was first predicted by the HartreeFock theory and has been confirmed as being the most probable outcome in various later, more detailed studies of the problem [320, 379, 380, 404]. In this case, the spin texture undergoes a $\pi$ phase shift across every charge stripe, so that every other spin stripe has the opposite Néel vector, cancelling out any magnetic intensity at the commensurate wavevector, $\langle\pi, \pi\rangle$. This situation [405, 406] has been called "topological doping." And, indeed, the predicted factor of two ratio between the spin and charge periodicities has been observed in all well established experimental realizations of stripe order in doped antiferromagnets. 47] Still, it is important to remember that nontopological stripes are also a logical possibility [379, 380, 396, 403, 407, 408], and we should keep our eyes open for this form of order, as well. ${ }^{37}$

In the context of frustrated phase separation, the formation of inhomo-

The Coulomb interaction sets the stripe spacing. geneous structures is predominantly a statement about the charge density, and its scale is set by the Coulomb interaction. This has several implications. Firstly, this means that charge stripes may begin to self-organize (at least locally) at relatively high temperatures, i.e. they are charge driven in the sense described above. 38 Secondly, charge density wave order always couples

$\overline{35}$ In the context of Landau-Ginzberg theory, the situation is somewhat more complex, and whether the spin and charge order have this relation, or have the same period turns out to depend on short distance physics, see footnote 37 and 403.

${ }^{36}$ Here, we exclude the possibility of perfectly circular spiral spin order, in which $\operatorname{Re}\{\langle\boldsymbol{S}\rangle\} \cdot \operatorname{Im}\{\langle\boldsymbol{S}\rangle\}=0$ and $[\operatorname{Re}\{\langle\boldsymbol{S}\rangle\}]^{2}=[\operatorname{Im}\{\langle\boldsymbol{S}\rangle\}]^{2} \neq 0$.

${ }^{37}$ For example, an analogous Landau theory of stripes near the Néel state must include the order parameter $\boldsymbol{S}_{\boldsymbol{\pi}}$, which favors in-phase domain walls 403.

38 In Hartree-Fock theory, stripes are spin driven. 
linearly to lattice distortions, so we should expect dramatic signatures of stripe formation to show up in the phonon spectrum. Indeed, phonons may significantly affect the energetics of stripe formation [409]. Thirdly, although we are used to thinking of density wave states as insulating, or at least as having a dramatically reduced density of states at the Fermi energy, this is not necessarily true. If the average hole concentration on each stripe is determined primarily by the competition between the Coulomb interaction and the local tendency to phase separation, the linear hole density per site along each stripe can vary as a function of $x$ and consequently there is no reason to expect the Fermi energy to lie in a gap or pseudogap. In essence, stripes may be intrinsically metallic, or even superconducting. Moreover, such compressible stripes are highly prone to lattice commensurability effects which tend to pin the inter-stripe spacing at commensurate values. Conversely, if the stripes are a consequence of some sort of Fermi surface nesting, as is the case in the Hartree-Fock studies [375, 378, 410] of stripe formation, the stripe period always adjusts precisely so as to maintain a gap or pseudogap at the Fermi surface: there is always one doped hole per site along each charge stripe. This insulating behavior is likely a generic feature of all local models of stripe formation 405], although more sophisticated treatments can lead to other preferred linear hole densities along a stripe [320,374].

In short, stripe order is theoretically expected to be a common form of self-organized charge ordering in doped antiferromagnets. In a $d$-dimensional striped state, the doped holes are concentrated in an ordered array of parallel $(d-1)$ dimensional hypersurfaces: solitons in $d=1$, "rivers of charge" in $d=$ 2 , and sheets of charge in $d=3$. This "charge stripe order" can either coexist with antiferromagnetism with twice the period (topological doping) or with the same period as the charge order, or the magnetic order can be destroyed by quantum or thermal fluctuations of the spins. Moreover, the stripes can be insulating, conducting, or even superconducting. It is important to recall that for $d<4$ quenched disorder is always a relevant perturbation for charge density waves, 411] so rather than stripe ordered states, real experiments may often require interpretation in terms of a "stripe glass" 412 415. Finally, for many purposes, it is useful to think of systems that are not quite ordered, but have substantial short range stripe order as low frequency fluctuations, as a "fluctuating stripe liquid". We will present an example of such a state in the next subsection.

\subsection{Avoided critical phenomena}

Let us examine a simple model of Coulomb frustrated phase separation. We seek to embody a system with two coexisting phases, which are forced to interleave due to the charged nature of one of the phases. To account for the short range tendency to phase separation, we include a short range "ferromagnetic" interaction which encourages nearest neighbor regions to be of the same phase, and also a long range "antiferromagnetic" interaction which
Competition sets the hole concentration on a stripe.

"Stripe glasses" and "stripe liquids" are also possible. 
prevents any domain from growing too large:

$$
H=-L \sum_{<i, j>} \boldsymbol{S}_{i} \cdot \boldsymbol{S}_{j}+\frac{Q a^{d-2}}{2} \sum_{i \neq j} \frac{\boldsymbol{S}_{i} \cdot \boldsymbol{S}_{j}}{\left|\boldsymbol{R}_{i}-\boldsymbol{R}_{j}\right|^{d-2}} .
$$

Here $\boldsymbol{S}_{j}$ is an $N$ component unit vector, $\boldsymbol{S}_{i} \cdot \boldsymbol{S}_{i}=1, L$ is a nearest neighbor ferromagnetic interaction, $Q$ is an antiferromagnetic "Coulomb" term which represents the frustration (and is always assumed small, $Q \ll L$ ), $d$ is the spatial dimension, $\langle i, j\rangle$ signifies nearest neighbor sites, $a$ is the lattice constant, and $\boldsymbol{R}_{j}$ is the location of lattice site $j$. The Ising $(N=1)$ version of this model is the simplest coarse grained model [358,416] of Coulomb frustrated phase separation, in which $S_{j}=1$ represents a hole rich, and $S_{j}=-1$ a hole poor region. In this case, $L>0$ is the surface tension of an interface between the two phases, and $Q$ is the strength of the Coulomb frustration. While the phase diagram of this model has been analyzed 416] at $T=0$, it is fairly complicated, and its extension to finite temperature has only been attempted numerically 417]. However, all the thermodynamic properties of this model can be obtained 418,419] exactly in the large $N$ limit.

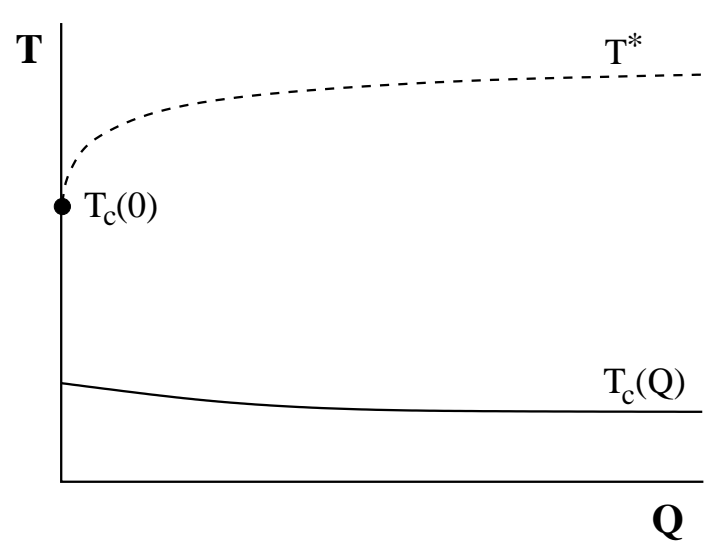

Fig. 46. Schematic phase diagram of the model in Eq. 146 of avoided critical phenomena. The thick black dot marks $T_{c}(Q=0)$, the ordering temperature in the absence of frustration; this is "the avoided critical point". Notice that $T_{c}(Q \rightarrow 0)<$ $T_{c}(Q=0)$. From Ref. 419 .

Fig. 46 shows the phase diagram for this model. Both for $Q=0$ and $Q \neq 0$, there is a low temperature ordered state, but the ordered state is fundamentally different for the two cases. For the unfrustrated case, the ordered state is homogeneous, whereas with frustration, there is an emergent length scale in the ordered state which governs the modulation of the order parameter. 
To be specific, in dimensions $d>2$ and for $N>2$, there is a low temperature ordered unidirectional spiral phase, which one can think of as a sort of stripe ordered phase 419]. Clearly, as $Q \rightarrow 0$, the modulation length scale must diverge, so that the homogeneous ordered state is recovered. However, like an antiferromagnet doped with neutral holes, there is a discontinuous change in the physics from $Q=0$ to any finite $Q$ : for $d \leq 3, \lim _{Q \rightarrow 0} T_{c}(Q) \equiv T_{c}\left(0^{+}\right)$is strictly less than $T_{c}(0)$. In other words, an infinitesimal amount of frustration depresses the ordering temperature discontinuously.

Although for any finite $Q$ the system does not experience a phase transition as the temperature is lowered through $T_{c}(0)$, the avoided critical point heavily influences the short range physics. For temperatures in the range $T_{c}(0)>T>T_{c}\left(0^{+}\right)$, substantial local order develops. An explicit expression for the spin-spin correlator can be obtained in this temperature range: At distances less than the correlation length $\xi_{0}(T)$ of the unfrustrated magnet, $R_{i j}<\xi_{0}(T)$, the correlator is critical,

$$
\left\langle\boldsymbol{S}_{i} \cdot \boldsymbol{S}_{j}\right\rangle \sim\left(a / R_{i j}\right)^{d-2-\eta}
$$

but for longer distances, $R_{i j}>\xi_{0}(T)$, it exhibits a damped version of the Goldstone behavior of a fluctuating stripe phase,

$$
\left\langle\boldsymbol{S}_{i} \cdot \boldsymbol{S}_{j}\right\rangle \sim\left(a / R_{i j}\right)^{\frac{d-1}{2}} \cos \left[K R_{i j}\right] \exp \left[-\kappa R_{i j}\right] .
$$

At $T_{c}(Q)$, the wavevector $K$ is equal to the stripe ordering wavevector of the low temperature ordered state, $K\left(T_{c}\right)=(Q / L)^{1 / 4}$. As the temperature is raised, $K$ decreases until it vanishes at a disorder line marked $T^{*}$ in the figure. The inverse domain size is given by

$$
\kappa(T)=\sqrt{(Q / L)^{1 / 4}-K^{2}(T)} .
$$

For a broad range of temperatures (which does not narrow as $Q \rightarrow 0$ ), this model is in a fluctuating stripe phase in a sense that can be made arbitrarily precise for small enough $Q$.

\subsection{The cuprates as doped antiferromagnets}

General considerations There is no question that the undoped parents of the high temperature superconductors are Mott insulators, in which the strong short range repulsion between electrons is responsible for the insulating behavior, and the residual effects of the electron kinetic energy (superexchange) lead to the observed antiferromagnetism. Indeed, one of the great theoretical triumphs of the field is the complete description, based on interacting spin waves and the resulting nonlinear sigma model, of the magnetism in these materials. 223, 224, 303

However, it is certainly less clear that one should inevitably view the superconducting materials as doped antiferromagnets, especially given that we

Our theoretical understanding of the undoped antiferromagnets is extolled. 
Why the cuprates should be viewed as doped antiferromagnets have presented strong reasons to expect a first order phase transition between $x=0$ and $x>0$. Nonetheless, many experiments on the cuprates are suggestive of a doped antiferromagnetic character. In the first place, various measurements of the density of mobile charge, including the superfluid density 107, 242, the "Drude weight" measured in optical conductivity 420, and the Hall number 421,422], are all consistent with a density proportional to the doped hole density, $x$, rather than the total hole density, $1+x$, expected from a band structure approach. Moreover, over a broad range of doping, the cuprates retain a clear memory of the antiferromagnetism of the parent correlated insulator. Local magnetism abounds. NMR, $\mu \mathrm{SR}$, and neutron scattering find evidence (some of which is summarized in Section 42) of static, or slowly fluctuating, spin patterns, including stripes, spin glasses, and perhaps staggered orbital currents. Static magnetic moments, or slowly fluctuating ones, are hard to reconcile with a Fermi liquid picture. There is also some evidence from STM of local electronic inhomogeneity 100, 101, 423 in BSCCO, indicative of the short range tendency to phase separate. The Fermi liquid state in a simple metal is highly structured in $k$-space, and so is highly homogeneous (rigid) in real space. This is certainly in contrast with experiments on the cuprates which indicate significant real space structure.

Stripes There is increasingly strong evidence that stripe correlations, as a specific feature of doped antiferromagnets, occur in at least some high temperature superconducting materials. The occurrence of stripe phases in the Another triumph of theory!

(Look, there are painfully few of them.) high temperature superconductors in particular, and in doped antiferromagnets more generally, was successfully predicted 39 by theory [375, 378, 410, Indeed, it is clear that a fair fraction of the theoretical inferences discussed in Section 12.2 are, at least in broad outline, applicable to a large number of materials, including at least some high temperature superconductors [6]. In particular, the seminal discovery 426] that in $\mathrm{La}_{1.6-x} \mathrm{Nd}_{0.4} \mathrm{Sr}_{x} \mathrm{CuO}_{4}$, first charge stripe order, then spin stripe order, and then superconductivity onset at successively lower critical temperatures is consistent with Coulomb frustrated phase separation. (See Fig. 47 in Section 42.) Somewhat earlier work on the closely related nickelates [427 established that the charge stripes are, indeed, antiphase domain walls in the spin order.

Controversy remains as to how universal stripe phases are in the cuprate superconducting materials, and even how the observed phases should be precisely characterized. This is also an exciting topic, on which there is con-

39 The theoretical predictions predated any clear body of well accepted experimental facts, although in all fairness it must be admitted that there was some empirical evidence of stripe-like structures which predated all of the theoretical inquiry: Even at the time of the first Hartree-Fock studies, there was already dramatic experimental evidence 424,425] of incommensurate magnetic structure in $\mathrm{La}_{2-x} \mathrm{Sr}_{x} \mathrm{CuO}_{4}$. 
siderable ongoing theoretical and experimental study. We will defer further discussion of this topic to Section 13.

\subsection{Additional considerations and alternative perspectives}

There are a number of additional aspects of this problem which we have not discussed here, but which we feel warrant a mention. In each case, clear discussions exist in the literature to which the interested reader is directed for a fuller exposition.

Phonons There is no doubt that strong electron-phonon coupling can drive a system to phase separate. Strong correlation effects necessarily enhance such tendencies, since they reduce the rigidity of the electron wavefunction to spatial modulation. (See, e.g., the 1D example in Section 9.2.) In particular, when there is already a tendency to some form of charge ordering, on very general grounds we expect it to be strongly enhanced by electron-phonon interactions.

This observation makes us very leery of any attempt at a quantitative comparison between results on phase separation or stripe formation in the $t-J$ or Hubbard models with experiments in the cuprates, where the electron-phonon interaction is manifestly strong 428. Conversely, there should generally be substantial signatures of various stripe-related phenomena in the phonon dynamics, and this can be used to obtain an experimental handle on these behaviors 160]. Indeed, there exists a parallel development of stripe-related theories of high temperature superconductivity based on Coulomb frustration of a phase separation instability which is driven by strong electron-phonon interactions [16, 61, 429]. The similarity between many of the notions that have emerged from these studies, and those that have grown out of studies of doped antiferromagnets illustrates both how robust the consequences of frustrated phase separation are in highly correlated systems, and how difficult it is to unambiguously identify a "mechanism" for it. For a recent discussion of many of the same phenomena discussed here from this alternative viewpoint, see Ref. 62 .

Spin-Peierls order Another approach to this problem, which emerges naturally from an analysis of the large $N$ limit [71], is to view the doped system as a "spin-Peierls" insulator, by which we mean a quantum disordered magnet in which the unit cell size is doubled but spin rotational invariance is preserved.10 While the undoped system is certainly antiferromagnetically ordered, it is argued that when the doping exceeds the critical value at which spin rotational symmetry is restored, the doped Mott insulating features of

$\overline{40}$ Alternatively, this state can be viewed as a bond-centered charge density wave [430, 431. 
the resulting state are better viewed as if they arose from a doped spin-Peierls state. Moreover, since the spin-Peierls state has a spin gap, it can profitably be treated as a crystal of Cooper pairs, which makes the connection to superconductivity very natural. Finally, as mentioned in Section [7, this approach has a natural connection with various spin liquid ideas.

Interestingly, it turns out that the doped spin-Peierls state also generically phase separates 390,432 434]. When the effect of long range Coulomb interactions are included, the result is a staircase of commensurate stripe phases 434. Again, the convergence of the pictures emerging from diverse starting points convinces us of the generality of stripey physics in correlated systems. For a recent discussion of the physics of stripe phases, and their connection to the cuprate high temperature superconductors approached from the large N/spin-Peierls perspective, see Ref. 435 .

Stripes in other systems It is not only the robustness of stripes in various theories that warrants mention, but also the fact that they are observed, in one way or another, in diverse physical realizations of correlated electrons. Stripes, and even a tendency to electronic phase separation, are by now well documented in the manganites - the colossal magnetoresistance materials. (For recent discussions, which review some of the literature, see Refs. 17, 436 and 108.) This system, like the nickelates and cuprates, is a doped antiferromagnet, so the analogy is quite precise.

Although the microscopic physics of quantum Hall systems is quite different from that of doped antiferromagnets, it has been realized for some time 437, 438 that in higher Landau levels, a similar drama occurs due to the interplay between a short ranged attraction and a long range repulsion between electrons which gives rise to stripe and bubble phases. Evidence of these, as well as quantum Hall nematic phases, [178, 439] has become increasingly compelling in recent years. (For a recent review, see 179.) On a more speculative note, it has been noticed that such behavior may be expected in the neighborhood of many first order transitions in electronic systems, and it has been suggested that various charge inhomogeneous states may play a role in the apparent metal-insulator transition observed in the two dimensional electron gas 440].

\section{Stripes and High Temperature Superconductivity}

We boast, and yet yearn for the unified understanding of $B C S$ theory.
In this article, we have analyzed the problem of high temperature superconductivity in a highly correlated electron liquid, with particular emphasis on doped antiferromagnets. We have identified theoretical issues, and even some solutions. We have also discussed aspects of the physics that elude a BCS description. This is progress.

However, we have not presented a single, unified solution to the problem. Contrast this with BCS, a theory so elegant it may captured in haiku: 


\author{
Instability \\ Of a tranquil Fermi sea - \\ Broken symmetry.
}

Of course, to obtain a more quantitative understanding of particular materials would require a few more verses - we might need to study the Eliashberg equations to treat the phonon dynamics in a more realistic fashion, and we may need to include Fermi liquid corrections, and we may also have to wave our hands a bit about $\mu^{*}$, etc. But basically, in the context of a single approximate solution of a very simple model problem, we obtain a remarkably detailed and satisfactory understanding of the physics. And while we may not be able to compute $T_{c}$ very accurately - it does, after all, depend exponentially on parameters - we can understand what sort of metals will tend to be good superconductors: metals with strong electron-phonon coupling, and consequently high room temperature resistances, are good candidates, as are metals with large density of states at the Fermi energy. We can also compute various dimensionless ratios of physical quantities, predict dramatic coherence effects (which do depend on microscopic details), and understand the qualitative effects of disorder.

The theory of high temperature superconductivity presented here reads more like a Russian novel, with exciting chapters and fascinating characters, but there are many intricate subplots, and the pages are awash in familiars, diminutives, and patronymics. To some extent, this is probably unavoidable. Fluctuation effects matter in the superconducting state: the phase ordering temperature, $T_{\theta}$, is approximately equal to $T_{c}$, and the zero temperature coherence length, $\xi_{0}$, is a couple of lattice constants. In addition, the existence of one or more physical pseudogap scales (the $T^{*}$ 's) in addition to $T_{c}$ means that there are multiple distinct qualitative changes in the physics in going from high temperature to $T=0$. Moreover, various other types of ordered states are seen in close proximity to or in coexistence with the superconducting state. Thus, it is more plausible that we will weave together a qualitative understanding of the basic physics in terms of a number of effective field theories, each capturing the important physics in some range of energy and length scales. Ideally, these different theories will be nested, with each effective Hamiltonian derived as the low energy limit of the preceding one.

While not as satisfying as the unified description of BCS-EliashbergMigdal theory, there is certainly ample precedent for the validity of this kind of multiscale approach. The number of quantitative predictions may be limited, but we should expect the approach to provide a simple understanding of a large number of qualitative observations. In fact, we may never be able to predict $T_{c}$ reliably, or even whether a particular material, if made, will be a good superconductor, but a successful theory should certainly give us some guidance concerning what types of new materials are good candidate high temperature superconductors [441, 442].

Before we continue, we wish to state a major change of emphasis. Up until
We outline a less ambitious goal for theory.

We now consider applicability. 
this point, we have presented only results that we consider to be on secure theoretical footing. That is, we have presented a valid theory. ${ }^{11}$ We now allow ourselves free rein to discuss the applicability of these ideas to the real world. In particular, we discuss the cuprate high temperature superconductors, and whether the salient physics therein finds a natural explanation in terms of stripes in doped antiferromagnets. Various open issues are laid out, as well as some general strategies for addressing them.

\subsection{Experimental signatures of stripes}

At the simplest level, stripes refer to a broken symmetry state in which the discrete translational symmetry of the crystal is broken in one direction: stripes is a term for a unidirectional density wave. "Charge stripes" refer to a unidirectional charge density wave (CDW). "Spin stripes" are unidirectional colinear spin density waves (SDW). ${ }^{42}$ More subtle local forms of stripes, such as stripe liquids, nematics and glasses are addressed in Section 13.2.

Where do stripes occur in the phase diagram? As discussed in Sections 11 and 12, holes doped into an antiferromagnet have a tendency to self-organize into rivers of charge, and these charge stripes tend to associate with antiphase domain walls in the spin texture. As shown in Section 12.2, stripe order is typically either "charge driven," in which case spin order onsets (if at all) at a temperature less than the charge ordering temperature, or "spin driven," if the charge order onsets as a weak parasitic order at the same temperature as the spin order. To the extent that stripes are indeed a consequence of Coulomb frustrated phase separation, we expect them to be charge driven, in this sense.

Neutron scattering has proven the most useful probe for unambiguously

Experimental evidence of stripes has been detected in: detecting stripe order. Neutrons can scatter directly from the electron spins. However, neutrons (and, for practical reasons, X-rays as well) can only detect charge stripes indirectly by imaging the induced lattice distortions. Alternatively, (as discussed in Section 12.2) since spin stripe order implies charge order, the magnetic neutron scattering itself can be viewed as an indirect measure of charge order. Since stripe order is unidirectional, it should ideally show up in a diffraction experiment as pairs of new Bragg peaks at

$\overline{41}$ High temperature superconductivity being a contentious field, it will not surprise the reader to learn that there is controversy over how important each of the issues discussed above is to the physics of the cuprates. As the field progresses, and especially as new data are brought to light, it may be that in a future version of this article we, too, might change matters of emphasis, but we are confident that no new understanding will challenge the validity of the theoretical constructs discussed until now.

42 Spiral SDW order has somewhat different character, even when unidirectional, and is not generally included in the class of striped states. 
positions $\boldsymbol{k}_{ \pm}=\boldsymbol{Q} \pm 2 \pi \hat{e} / \lambda$ where $\hat{e}$ is the unit vector perpendicular to the stripe direction, $\lambda$ is the stripe period, and $\boldsymbol{Q}$ is an appropriate fiduciary point. For charge stripes, $\boldsymbol{Q}$ is any reciprocal lattice vector of the underlying crystal, while for spin stripes, $\boldsymbol{Q}$ is offset from this by the Néel ordering vector, $\langle\pi, \pi\rangle$. Where both spin and charge order are present, the fact that the charge stripes are associated with magnetic antiphase domain walls is reflected in the fact that $\lambda_{\text {spin }}=2 \lambda_{\text {charge }}$, or equivalently $\boldsymbol{k}_{\text {charge }}=2 \boldsymbol{k}_{\text {spin }}$.

$\mathrm{La}_{1.6-x} \mathrm{Nd}_{0.4} \mathrm{Sr}_{x} \mathrm{CuO}_{4}$ (LNSCO) is stripe ordered, and the onset of stripe ordering with temperature is clear. Fig. 47 shows data from neutron scattering, NQR, and susceptibility measurements 413]. In this material, charge stripes form at a higher temperature than spin stripes. Note also that static charge and spin stripes coexist with superconductivity throughout the superconducting dome. In fact experiments reveal quartets of new Bragg peaks, at $\boldsymbol{Q} \pm 2 \pi \hat{x} / \lambda$ and $\boldsymbol{Q} \pm 2 \pi \hat{y} / \lambda$. In this material, the reason for this is understood to be a bilayer effect - there is a crystallographically imposed tendency for the stripes on neighboring planes to be oriented at right angles to each other, giving rise to two equivalent pairs of peaks. Charge and spin peaks have also been detected 443 in neutron scattering studies of $\mathrm{La}_{1.875} \mathrm{Ba}_{0.125-x} \mathrm{Sr}_{x} \mathrm{CuO}_{4}$.

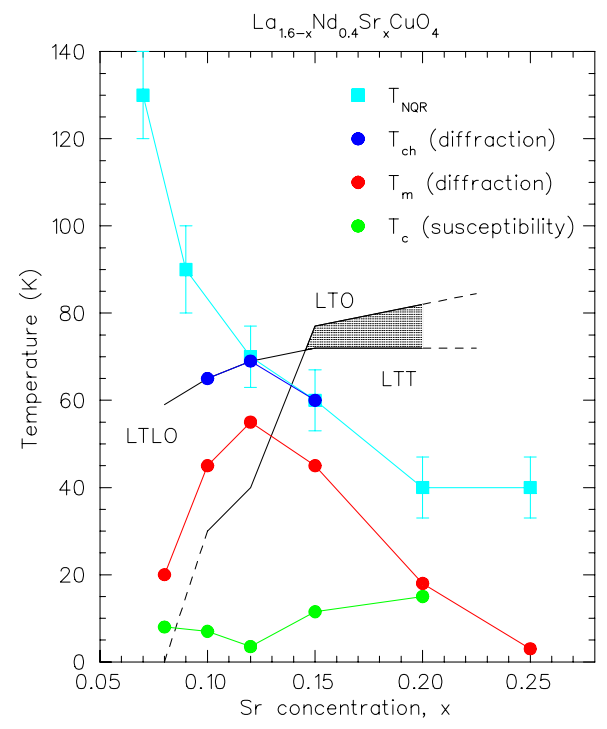

Fig. 47. Blue data points refer to the onset of charge inhomogeneity. Red data points denote the onset of incommensurate magnetic peaks. Green data points are the superconducting $T_{c}$. From Ichikawa et al. 413

Spin stripe order has also been observed from elastic neutron scattering in $\mathrm{La}_{2-x} \mathrm{Sr}_{x} \mathrm{CuO}_{4}$ (LSCO) for dopings between $x=.02$ and $x=.05$ where the material is not superconducting at any $T$; these stripes are called 
diagonal, because they lie along a direction rotated $45^{\circ}$ to the $\mathrm{Cu}-\mathrm{O}$ bond direction [164]. Above $x=.05$ [444], the stripes are vertical43, i.e. along the $\mathrm{Cu}-\mathrm{O}$ bond direction, and the samples are superconducting at low temperature. For dopings between $x=.05$ and $x=.13$, the stripes have an ordered (static) component. In the region $x=.13$ to $x=.25$, incommensurate magnetic peaks have been detected with inelastic neutron scattering. Because of the close resemblance between these peaks and the static order observed at lower doping, this can be unambiguously interpreted as being due to slowly fluctuating stripes.

Neutron scattering has also detected spin stripes in $\mathrm{La}_{2} \mathrm{CuO}_{4+\delta}$ (LCO) with $\delta=.12$ 445. In this material, static stripes coexist with superconductivity even at optimal doping. In the $T_{c}=42 \mathrm{~K}$ samples (the highest $T_{c}$ for this family thus far), superconductivity and spin stripe order onset simultaneously [166, 445]. Application of a magnetic field suppresses the superconducting transition temperature, but has little effect on the ordering temperature of the spins 4446].

In very underdoped nonsuperconducting LSCO, because the stripes lie along one of the orthorhombic axes, it has been possible to confirm 447, 448 that stripe order leads, as expected, to pairs of equivalent Bragg spots, indicating unidirectional density wave order. In both superconducting LSCO and LCO, quartets of equivalent Bragg peaks are observed whenever stripe order occurs. This could be due to a bilayer effect, as in LNSCO, or due to a large distance domain structure of the stripes within a given plane, such that different domains contribute weight to one or the other of the two pairs of peaks. However, because the stripe character in these materials so closely resembles that in LNSCO, there is no real doubt that the observed ordering peaks are associated with stripe order, as opposed to some form of checkerboard order.

In $\mathrm{YBaCu}_{2} \mathrm{O}_{6+y}(\mathrm{YBCO})$, incommensurate spin fluctuations have been identified throughout the superconducting doping range. [145, 160, 163, 449] By themselves, these peaks (which are only observed at frequencies above a rather substantial spin gap) are subject to more than one possible interpretation [450], although their similarity [451] to the stripe signals seen in LSCO is strong circumstantial evidence that they are associated with stripe fluctuations. Recently, this interpretation has been strongly reinforced by several additional observations. Neutron scattering evidence [163] has been found of static charge stripe order in underdoped YBCO with $y=.35$ and $T_{c}=39 \mathrm{~K}$. The charge peaks persist to at least $300 \mathrm{~K}$. The presence of a static stripe phase in YBCO means that inelastic peaks seen at higher doping are very likely fluctuations of this ordered phase. In addition, phonon anomalies have

$\overline{43}$ We should say mostly vertical. Careful neutron scattering work 165, 445] on LSCO and LCO has shown that the incommensurate peaks are slightly rotated from the $\mathrm{Cu}-\mathrm{O}$ bond direction, corresponding to the orthorhombicity of the crystal. 
been linked to the static charge stripes at $y=.35$, and used to detect charge fluctuations at $y=.6[160$. By studying a partially detwinned sample with $y=.6$, with a $2: 1$ ratio of domains of crystallographic orientation, Mook and collaborators were able to show that the quartet of incommensurate magnetic peaks consists of two inequivalent pairs, also with a 2:1 ratio of intensities in the two directions [452]. This confirms that in YBCO, as well, the signal arises from unidirectional spin and charge modulations (stripes), and not from a checkerboard-like pattern.

Empirically, charge stripe formation precedes spin stripe formation as the temperature is lowered, and charge stripes also form at higher temperatures than $T_{c}$. Both types of stripe formation may be a phase transition, or may simply be a crossover of local stripe ordering, depending upon the material and doping. Where it can be detected, charge stripe formation occurs at a higher temperature than the formation of the pairing gap, ${ }_{4}$ consistent with the spin gap proximity effect (see Section 10.4).

Although some neutron scattering has been done on $\mathrm{Bi}_{2} \mathrm{Sr}_{2} \mathrm{CaCu}_{2} \mathrm{O}_{8+\delta}$ (BSCCO), the probe has only produced weak evidence of significant incommensurate structure [453. The weak coupling of planes in BSCCO makes neutron scattering difficult, as it is difficult to grow the requisite large crystals. However, BSCCO is very well suited to surface probes such as ARPES and STM. Recent STM data, both with 454] and without 455 an external magnetic field have revealed a static modulation in the local density of states that is very reminiscent of the incommensurate peaks observed with neutron scattering. Indeed, in both cases, the Fourier transform of the STM image exhibits a clear quartet of incommensurate peaks, just like those seen in neutron scattering in LSCO and YBCO. Here, however, unlike in the neutron scattering data, phase information is available in that Fourier transform. Using standard image enhancement methods, this phase information can be exploited 455 to directly confirm that the quartet of intensity peaks is a consequence of a domain structure, in which the observed density of states modulation is locally one dimensional, but with an orientation that switches from domain to domain. The use of STM as a probe of charge order is new, and there is much about the method that needs to be better understood 4456 before definitive conclusions can be reached, but the results to date certainly look very promising.

Finally, striking evidence of electronic anisotropy has been seen in untwinned crystals of $\mathrm{La}_{2-x} \mathrm{Sr}_{x} \mathrm{CuO}_{4}(x=0.02-0.04)$ and $\mathrm{YBa}_{2} \mathrm{CuO}_{6+y}$ $(y=0.35-1.0)$ by Ando and collaborators 987. The resistivity differs in the two in-plane directions in a way that cannot be readily accounted for by crystalline anisotropy alone. It is notable that in $\mathrm{YBCO}$, the anisotropy increases as $y$ is decreased. That is, the electrical anisotropy increases as the orthorhombicity is reduced. In some cases, substantial anisotropy persists up to temperatures as high as 300K. Furthermore, for $y<0.6$, the anisotropy

\footnotetext{
${ }^{44}$ See our discussion of the pseudogap(s) in Section 3.5.
}

Preliminary evidence of nematic order has been detected, as well. 
increases with decreasing temperature, much as would be expected 445] for an electron nematic. These observations from transport correlate well with the evidence from neutron scattering [452], discussed above, of substantial orientational order of the stripe correlations in YBCO, and with the substantial, and largely temperature independent anisotropy of the superfluid density observed in the same material. [13] Together, these observations constitute important, but still preliminary evidence of a nematic stripe phase in the cuprates.

\subsection{Stripe crystals, fluids, and electronic liquid crystals}

Stripe ordered phases are precisely defined in terms of broken symmetry. A charge stripe phase spontaneously breaks the discrete translational symmetry and typically also the point group symmetry (e.g. four-fold rotational symmetry) of the host crystal. A spin stripe phase breaks spin rotational symmetry as well. While experiments to detect these orders in one or another specific material may be difficult to implement for practical reasons and because of the complicating effects of quenched disorder, the issues are unambiguous. Where these broken symmetries occur, it is certainly reasonable to conclude that the existence of stripe order is an established fact. That this can be said to be the case in a number of superconducting cuprates is responsible for the upsurge of interest in stripe physics.

It is much more complicated to define precisely the intuitive notion of a "stripe fluid" 15 Operationally, it means there is sufficient short ranged stripe order that, for the purposes of understanding the mesoscale physics, it is possible to treat the system as if it were stripe ordered, even though translational symmetry is not actually broken. It is possible to imagine intermediate stripe liquid phases which are translationally invariant, but which still break some symmetries which directly reflect the existence of local stripe order. The

Some stripe liquids break rotational symmetry.

Melting stripes simplest example of this is an "electron nematic" phase. In classical liquid crystals, the nematic phase occurs when the constituent molecules are more or less cigar shaped. It can be thought of as a phase in which the cigars are preferentially aligned in one direction, so that the rotational symmetry of free space is broken (leaving only rotation by $\pi$ intact) but translational symmetry is unbroken. In a very direct sense, this pattern of macroscopic symmetry breaking is thus encoding information about the microscopic constituents of the liquid. In a similar fashion, we can envisage an electron nematic as consisting of a melted stripe ordered phase in which the stripes meander, and even break into finite segments, but maintain some degree of orientational order-for instance, the stripes are more likely to lie in the $\mathrm{x}$ rather than the y direction; see Fig. 48.

One way to think about different types of stripe order is to imagine starting with an initial "classical" ordered state, with coexisting unidirec-

${ }^{45}$ For the present purposes, the term "fluctuating stripes" is taken to be synonymous with a stripe fluid. See, for example, Refs. 405 and 458 . 

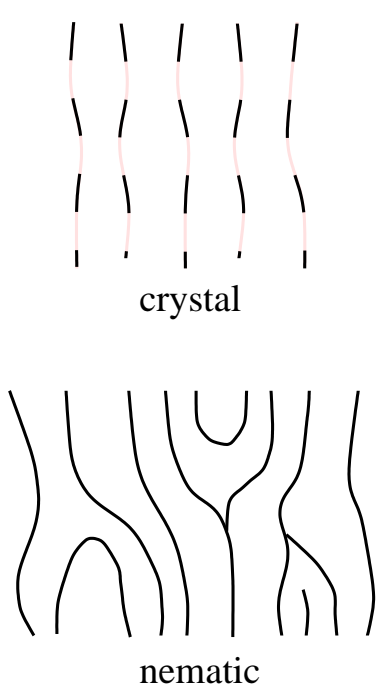
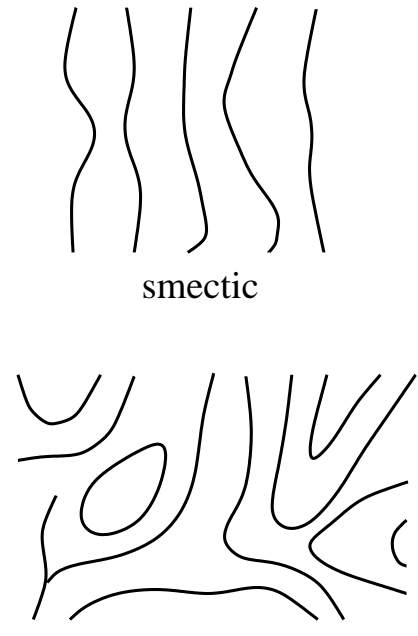

isotropic

Fig. 48. Schematic representation of various stripe phases in two dimensions. The broken lines represent density modulations along the stripes. In the electronic crystal, density waves on neighboring stripes are locked in phase and pinned. The resulting state is insulating and breaks translation symmetry in all directions. Solid lines represent metallic stripes along which electrons can flow. They execute increasingly violent transverse fluctuations as the system is driven towards the transition into the nematic phase. The transition itself is associated with unbinding of dislocations that are seen in the snapshot of the nematic state. The isotropic stripe fluid breaks no spatial symmetries of the host crystal, but retains a local vestige of stripe order.

tional SDW and CDW order. As quantum fluctuations are increased (metaphorically, by increasing $\hbar$ ), one can envisage that the soft orientational fluctuations of the spins will first cause the spin order to quantum melt, while the charge order remains. If the charge order, too, is to quantum melt in a continuous phase transition, the resulting state will still have the stripes generally oriented in the same direction as in the ordered state, but with unbound dislocations which restore translational symmetry. ${ }^{\text {ml }}$ If the underlying crystal is tetragonal 463], this state still spontaneously breaks the crystal point group symmetry. In analogy with the corresponding classical state, it has been called an electron nematic, but it could also be viewed as an electronically driven orthorhombicity. This is still a state with broken symmetry, so in principle its existence should be unambiguously identifiable from experi-

$\overline{46}$ It is also possible to view the electron nematic from a weak coupling perspective, where it occurs as a Fermi surface instability 459, sometimes referred to as a Pomeranchuk instability. [460, 461] This instability is "natural" when the Fermi surface lies near a Van Hove singularity. The relation between the weak coupling and the stripe fluid pictures is currently a subject of ongoing investigation 462]. 
ment. ${ }^{47}$ The order parameter can be identified with the matrix elements of any traceless symmetric tensor quantity, for instance the traceless piece of the dielectric or conductivity tensors.

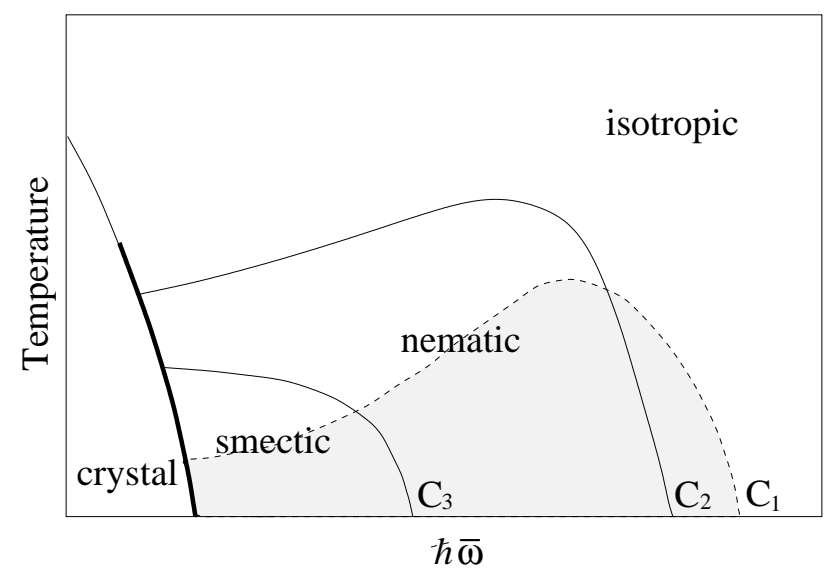

Fig. 49. Schematic phase diagram of a fluctuating stripe array in a (tetragonal) system with four-fold rotational symmetry in $D=2$. Here $\hbar \bar{\omega}$ is a measure of the magnitude of the transverse zero point stripe fluctuations. Thin lines represent continuous transitions and the thick line a first order transition. We have assumed that the superconducting susceptibility on an isolated stripe diverges as $T \rightarrow 0$, so that at finite stripe density, there will be a transition to a globally superconducting state below a finite transition temperature. On the basis of qualitative arguments, discussed in the text, we have sketched a boundary of the superconducting phase, indicated by the shaded region. Depending on microscopic details the positions of the quantum critical points $C_{1}$ and $C_{2}$ could be interchanged. Distinctions between various possible commensurate and incommensurate stripe crystalline and smectic phases are not indicated in the figure. Similarly, all forms of spin order are neglected in the interest of simplicity.

With this physics in mind, we have sketched a qualitative phase diagram, shown in Fig. 49, which provides a physical picture of the consequences of melting a stripe ordered phase. As a function of increasing quantum and thermal transverse stripe fluctuations one expects the insulating electronic

$\overline{47}$ It is probable that when nematic order is lost, the resulting stripe liquid phase is not thermodynamically distinct from a conventional metallic phase, although the local order is sufficiently different that one might expect them to be separated by a first order transition. However, it is also possible that some more subtle form of order could distinguish a stripe liquid from other electron liquid phases - for instance, it has been proposed by Zaanen and collaborators [464] that a stripe liquid might posses an interesting, discrete topological order which is a vestige of the antiphase character of the magnetic correlations across a stripe. 
crystal, which exists at low temperatures and small $\hbar$, to evolve eventually into an isotropic disordered phase. At zero temperature this melting occurs in a sequence of quantum transitions [52]. The first is a first order transition into a smectic phase, then by dislocation unbinding a continuous transition leads to a nematic phase that eventually evolves (by a transition that can be continuous in $D=2$, but is first order in a cubic system) into the isotropic phase. Similar transitions exist at finite temperature as indicated in Fig. 49.

We have also sketched a superconducting phase boundary in the same figure. Provided that there is a spin gap on each stripe, and that the charge Luttinger exponent $K_{c}>1 / 2$, then (as discussed in Section 5) there is a divergent superconducting susceptibility on an isolated stripe. In this case, the superconducting $T_{c}$ is determined by the Josephson coupling between stripes. Since, as discussed in Section 6 , the mean Josephson coupling increases with increasing stripe fluctuations, $T_{c}$ also rises with increasing $\hbar$ throughout the smectic phase. While there is currently no well developed theory of the superconducting properties of the nematic phase, ${ }^{18}$ to the extent that we can think of the nematic as being locally smectic, it is reasonable to expect a continued increase in $T_{c}$ across much, or all of the nematic phase, as shown in the figure. However, as the stripes lose their local integrity toward the transition to the isotropic phase we expect, assuming that stripes are essential to the mechanism of pairing, that $T_{c}$ will decrease, as shown.

The study of electronic liquid crystalline phases is in its infancy. Increasingly unambiguous experimental evidence of the existence of nematic phases has been recently reported in quantum Hall systems [178, 180, 1339, 457, 466] in addition to the preliminary evidence of such phases in highly underdoped cuprates discussed above. Other more exotic electronic liquid crystalline phases are being studied theoretically. This is a very promising area for obtaining precise answers to well posed questions that may yield critical information concerning the important mesoscale physics of the high temperature superconductors.

\subsection{Our view of the phase diagram-Reprise}

Since the motion of dilute holes in a doped antiferromagnet is frustrated, the minimization of their kinetic energy is a complicated, multistage process. We have argued that this is accomplished in three stages: (a) the formation of static or dynamical charge inhomogeneity (stripes) at $T_{\text {stripe }}^{*}$ (b) the creation of local spin pairs at $T_{\text {pair }}^{*}$, which creates a spin gap, and (c) the establishment of a phase-coherent superconducting state at $T_{c}$. The zero point kinetic energy is lowered along a stripe in the first stage, and perpendicular to the stripe in the second and third stages. Steps (a), (b), and (c) above are clearcut only if the energy scales are well separated, that is, if $T_{\text {stripe }}^{*}>>T_{\text {pair }}^{*}>>T_{c}$. On the

$\overline{48}$ Some very promising recent progress toward developing a microscopic theory of the electron nematic phase has been reported in Refs. 459 and 465 .
Superconducting electronic liquid crystals

It's all about kinetic energy. 
underdoped side at least, if we identify $T_{\text {stripe }}^{*}$ and $T_{\text {pair }}^{*}$ with the appropriate observed pseudogap phenomena (see Section 3.5) there is a substantial (if not enormous) separation of these temperature scales.

Pseudogap scales At high temperatures, the system must be disordered. As temperature is lowered, the antiferromagnet ejects holes, and charge stripe correlations develop. This may be either a phase transition or a crossover. We have called this temperature $T_{\text {stripe }}^{*}$ in Fig. 12. Even if it is a phase transition, for instance a transition to a stripe nematic state, local order may develop above the ordering temperature, and probes on various time scales may yield different answers for $T_{\text {stripe }}^{*}$. As the antiferromagnet ejects holes, local antiferromagnet correlations are allowed to develop. Probes bearing on this temperature include the Knight shift, NQR, and diffraction. At a lower temperature, through communication with the locally antiferromagnetic environment, a spin gap develops on stripes. We identify this spin gap with the pairing gap, and have labeled this temperature (which is always a crossover) $T_{\text {pair }}^{*}$. Probes bearing on this temperature measure the single particle gap, and include ARPES, tunnelling, and NMR.

Dimensional crossovers are a necessary consequence of stripe physics.
Dimensional crossovers Looking at this evolution from a broader perspective, there are many consequences that can be understood based entirely on the notion that the effective dimensionality of the coherent electronic motion is temperature dependent. At high temperatures, before local stripe order occurs, the electronic motion is largely incoherent-i.e the physics is entirely local. Below $T_{\text {stripe }}^{*}$, the motion crosses over from quasi 0D to quasi 1D behavior.19 Here, significant $\boldsymbol{k}$ space structure of various response functions is expected, and there may well emerge a degree of coherence and possibly pseudogaps, but the electron is not an elementary excitation, so broad spectral functions and non-Fermi liquid behavior should be the rule. Then, at a still lower temperature, a 1D to 3D crossover occurs as coherent electronic motion between stripes becomes possible. At this point coherent quasiparticles come to dominate the single particle spectrum, and more familiar metallic and/or superconducting physics will emerge. If the spin gap is larger than this crossover temperature (as it presumably is in underdoped materials), then this crossover occurs in the neighborhood of $T_{c}$. However, if the spin gap is small, then the dimensional crossover will likely occur at temperatures well above $T_{c}$, and $T_{c}$ itself will have a more nearly BCS character, as discussed in Section 5.3 this seems to be crudely what happens in the overdoped materials 470]. Since once there are well developed quasiparticles,

$\overline{49}$ It is intuitively clear that kinetic energy driven stripe formation should lead to increased hole mobility, as is observed, but how the famous $T$-linear resistivity can emerges from local quasi-0D physics is not yet clear. See, however, Refs. 358 , 467469. 
there is every reason to expect them to be able to move coherently between planes, there is actually no substantial region of quasi 2D behavior expected. Although it may be hard, without a macroscopically oriented stripe array, to study the dimensional crossover by measuring in-plane response functions, the dimensional crossover can be studied by comparing in-plane to out-of plane behavior. 50

The cuprates as quasi-1D superconductors When $T_{\text {stripe }}^{*}>>T_{\text {pair }}^{*}>>$ $T_{c}$, the model of a quasi-one dimensional superconductor introduced in Section 5.3 is applicable in the entire temperature range below $T_{\text {stripe }}^{*}$. The application of these results to the overdoped side is suspect, since that is where all of these energy scales appear to crash into each other.

The temperature dependence of the spectral response of a quasi-one dimensional superconductor may be described as follows: At temperatures high compared to both the Josephson coupling and the spin gap, the system behaves as a collection of independent (gapless) Luttinger liquids. Spin-charge separation holds, so that an added hole dissolves into a spin part and a charge part. Consequently the spectral response exhibits broad EDC's and sharp MDC's. 51 In the intermediate temperature regime (below the spin gap), spin-charge separation still holds, and the ARPES response still exhibits fractionalized spectra, but with a pseudogap. In the low temperature phase, Josephson coupling between stripes confines spin and charge excitations, restoring the electron as an elementary excitation, and a sharp coherent peak emerges from the incoherent background, with weight proportional to the coupling between stripes.

There is a wealth of ARPES data on BSCCO, a material which lends itself more to surface probes than to diffraction. However, as mentioned previously, the presently available evidence of stripes in this material is compelling, but not definitive, so it requires a leap of faith to interpret the ARPES data in terms of stripes. The best evidence of stripes comes from STM data which is suggestive of local stripe correlations 454,455. Since STM observes a static modulation, any stripes observed in STM can certainly be considered static as far as ARPES is concerned. 52 As long as the stripes have integrity over a length scale at least as large as $\xi_{s}=v_{s} / \Delta_{s}$, it is possible for the stripes to support superconducting pairing through the spin gap proximity effect.

50 Much of the successful phenomenology of dimensional crossover developed in conjunction with the interlayer pairing mechanism of superconductivity [21] is explained in this way in the context of a stripe theory.

51 See Section for a description of EDC's and MDC's.

52 Unfortunately,there is currently little direct experimental information concerning the temperature dependence of the stripe order in BSCCO, although what neutron scattering evidence does exist [453], suggests that substantial stripe correlations survive to temperatures well above the superconducting $T_{c}$.

ARPES and stripes

ARPES spectra from the antinodal region resemble a quasi-1D superconductor. 
Stripes and superconductivity involve the same regions of $k$-space
At any rate, many features of the ARPES spectra, especially those for $\boldsymbol{k}$ in the antinodal region of the Brillouin zone (near $(\pi, 0)$ ) in BSCCO are unlike anything seen in a conventional metal, and highly reminiscent of a quasi-1D superconductor. Above $T_{c}$, ARPES spectra reveal sharp MDC's and broad EDC's. We take this [36] as evidence of electron fractionalization. Below $T_{c}$, a well defined quasiparticle peak emerges [89], whose features are strikingly similar to those derived in this model. The quasiparticle peak is nearly dispersionless along the $(0,0)$ to $(\pi, 0)$ direction, and within experimental bounds its energy and lifetime are temperature independent. The only strongly temperature dependent part of the spectrum is the intensity associated with the superconducting peak. The temperature dependence of the intensity is consistent with its being proportional to a fractional power of the local condensate fraction or the superfluid density. Similar behavior has been measured now in an untwinned single crystal of YBCO [12] as well.

The most dramatic signatures of superconducting phenomena in ARPES experiments, both the development of the gap and the striking onset of the superconducting peak with phase coherence, occur in the same regions of $k$-space most associated with stripes: Specifically, an array of "horizontal" charge stripes embedded in a locally antiferromagnetic environment 471 474] has most of its low energy spectral weight concentrated near the $(\pi, 0)$ regions of $k$-space. Similarly, the strongest gap develops in the $(\pi, 0)$ regions, and in both BSCCO and YBCO, the only dramatic change in the ARPES response upon entering the superconducting state is the coherent peak seen in these same regions.

The ARPES spectrum from the nodal region $(\boldsymbol{k}$ near $(\pi / 2, \pi / 2))$ is less obviously one dimensional in character, although the nodal spectrum is certainly consistent with the existence of stripes, as has been demonstrated in several model calculations 133, 471 473, 475. However, to a large extent, the spectrum in the nodal region is insensitive to stripe correlations. 1233. Nodal quasiparticles are certainly important for the low temperature properties of the superconducting state. Moreover, there is indirect evidence that they dominate the in-plane transport above $T_{c}$. But the fact that the ARPES spectrum in the nodal direction does not change 476] in any dramatic fashion from above to below $T_{c}$, as one would have deduced even from the simplest BCS considerations, suggests that they do not play a direct role in the mechanism of superconductivity. This observation, however, must not be accepted unconditionally. There is an apparent contradiction between the smooth evolution of the spectral function observed in ARPES and the evolution inferred from macroscopic transport experiments 477, 478; the latter suggest that a catastrophic change in the nodal quasiparticle lifetime occurs in the immediate neighborhood of $T_{c}$.

Inherent competition Finally, it should be made clear that a stripes based mechanism of high temperature superconductivity predicts competition be- 
tween stripes and superconductivity: static stripes may be good for pairing, but are certainly bad for the Josephson coupling (superfluid stiffness) between stripes. On the other hand, fluctuating stripes produce better Josephson coupling, but weaker pairing. The dependence of the gap on stripe fluctuations finds its origin in the spin gap proximity effect, where the development of the spin gap hinges on the one dimensionality of the electronic degrees of freedom [20], whereas stripe fluctuations cause the system to be more two dimensional. In addition, as described in Section 6, stripe fluctuations work against $2 k_{F}$ CDW order along a stripe, but strengthen the Josephson coupling.

This is consistent with the empirical phase diagram: on the underdoped side there is a large gap, small superfluid stiffness, small transition temperature, and static stripes have been observed. With increasing doping, stripes fluctuate more, reducing the pairing gap, but increasing the Josephson coupling between stripes. This is a specific example of the doping dependent crossover scenario proposed in Refs. 110,269, in which underdoped cuprates have a strong pairing scale but weak phase stiffness and $T_{c}$ is determined more or less by $T_{\theta}$, whereas the overdoped cuprates have a strong phase stiffness but weak pairing scale and $T_{c}$ is more closely associated with $T_{\text {pair }}^{*}$. Optimal doping is a crossover between a dominantly phase ordering transition and a dominantly pairing transition.

\subsection{Some open questions}

As has been stressed by many authors, the cuprate superconductors are exceedingly complex systems. Crisp theoretical statements can be made concerning the behavior of simplified models of these systems, but it is probably ultimately impossible to make clean predictions about whether the results will actually be found in any given material. We are therefore reliant on experiment to establish certain basic empirical facts. In this subsection, we will discuss some of the fundamental issues of fact that are pertinent to the stripe scenario presented above, and make a few comments about the present state of knowledge concerning them. A word of caution is in order before we begin: positive results have clearer implications than negative results. Especially in these complicated materials, there can be many reasons to fail to see an effect.

Are stripes universal in the cuprate superconductors? If stripes are not, in some sense, universal in the high temperature superconductors, then they cannot be, in any sense, essential to the mechanism of high temperature superconductivity. So an important experimental issue is whether stripes are universal in the cuprate superconductors.

The evidence from neutron scattering is discussed in Section 42: Incommensurate (IC) spin peaks (whether elastic or inelastic) have been detected throughout the doping range of superconductivity in the lanthanum compounds. In YBCO, IC spin peaks are seen with inelastic scattering, but it is
We, too, think stripes compete with superconductivity.

Concerning negative results: "Accentuate the positive." 
presently unclear how much of that scattering intensity should be associated with stripe fluctuations, and how much should be associated with the "resonance peak". Neutron scattering has produced some evidence 453] of IC spin peaks in BSCCO, but this result is controversial [479]. No such peaks have been reported in $\mathrm{TlBaCaCuO}$ or $\mathrm{HgBaCaCuO}$, although little or no neutron scattering has yet been done on crystals of these materials.

CDW order turns out to be much harder to observe, even when we know it is there. Charge stripe order has only been observed directly in $\mathrm{La}_{1.6-x} \mathrm{Nd}_{0.4} \mathrm{Sr}_{x} \mathrm{CuO}_{4}$ 413] and very underdoped $\mathrm{YBa}_{2} \mathrm{Cu}_{3} \mathrm{O}_{7-\delta}$ [163], although the general argument presented in Section 12.2 implies that it must occur wherever spin stripe order exists. Given the difficulty in observing the charge order where we know it exists, we consider an important open question to be: Where does charge stripe order exist in the general phase diagram of the cuprate superconductors?

As mentioned before, STM experiments point to local charge stripes in BSCCO, both with [454] and without [455] a magnetic field. But there is nowhere near enough systematic data to know whether charge stripes are ubiquitous as a function of doping and in all the superconducting cuprates, how pronounced it is, and over what range of temperatures significant stripe correlations exist, even where we know they exist at low temperatures. Perhaps, in the future, this issue can be addressed further with STM, or even with ARPES or new and improved X-ray scattering experiments.

Are stripes an unimportant low temperature complication? There is a general tendency for increasingly subtle forms of order to appear as systems are cooled - involving residual low energy degrees of freedom that remain after the correlations that are the central features of the physics have developed. (A classic example of this is transitions involving ordering of the nuclear moments at ultra-low temperatures in a metal.) While such forms of order are fascinating in their own right, one would not, typically, view them as important aspects of the basic materials physics of the studied system. There is a body of thought that holds that the various forms of stripe order that have been observed are in this class of phenomena-interesting side shows, but not the main event. It is also true that actual, static stripe order has only been observed under rather restrictive conditions - mostly in highly underdoped materials or materials with significantly depressed superconducting $T_{c}$ 's, and at temperatures less than or of order the optimal superconducting $T_{c}$.

To be central to the physics of high temperature superconductivity, charge stripes must occur at high enough energies and temperatures that they are relevant to zeroth order. Specifically, we want to look for evidence that local stripes persist up to temperatures which are greater than or equal to $T_{c}$. If stripes are universal, then there must be a characteristic crossover scale below which significant stripe correlations emerge - clearly, at high enough temperature, no significant self-organization is possible. Undoubtedly, there 
is a high energy scale associated with one or more pseudogap crossovers in many underdoped materials - can we associate some of this crossover with the scale at which local stripe correlations become significant? If so, then manifestly stripes are a central player in the drama. If not, and if no still higher energy scale can be identified at which stripe physics begins, it would become increasingly difficult to envisage a starring role for stripes in the physics of the cuprates.

This issue has not been unambiguously resolved. There is substantial (yet not definitive) evidence that local stripe order persists to rather high temperatures. Evidence of local stripe order from observed [480] infrared active phonon modes has been seen to persist to at least $300 \mathrm{~K}$ in highly underdoped $\mathrm{La}_{2-x} \mathrm{Sr}_{x} \mathrm{CuO}_{4}$. Phonon anomalies, which have been tentatively associated with stripes, have been observed in neutron scattering experiments in slightly underdoped $\mathrm{YBa}_{2} \mathrm{Cu}_{3} \mathrm{O}_{7-\delta}$ up to comparable temperatures [160]. Still more indirect evidence also abounds. This is a key question, and much more work is necessary to resolve it.

Are the length and time scales reasonable? As emphasized above, to understand the mechanism of high temperature superconductivity, we are primarily concerned with mesoscopic physics, on length scales a few times the superconducting coherence length and time scales a few times $\hbar / \Delta_{0}$. So the real question we want to address is: Does stripe order exist on these length and time scales? Given that it is so difficult to determine where long range charge stripe order occurs, it is clearly still more complicated to determine where substantial stripey short range order occurs, or even precisely how much short range order is sufficient.

Are stripes conducting or insulating? The earliest theoretical studies which predicted stripes as a general feature of doped antiferromagnets envisaged insulating stripes $375,377,378$. These stripes are conceptually close relatives of conventional CDW's in that they are obtained as a Fermi surface instability due to near perfect nesting of the Fermi surface. Such stripes have no low lying fermionic excitations. This perspective has led to an interesting theory of superconductivity which relies on stripe defects for charge transport 481.

The strongest evidence that charge stripes are incompressible, and therefore insulating, comes from plotting the magnetic incommensurability against the doping concentration. If this relationship is strictly linear, it implies that the concentration of holes on a stripe does not change, but rather the only effect of further doping is to change the concentration of stripes in a plane, bringing the stripes closer together. The data for LSCO are close to linear in the range $.024 \leq x \leq .12$, despite the change in orientation from diagonal to vertical at $x=.05,167,482$ but the small deviation from linearity below 
$x=.06$ does exceed the error bars. At present, the data leave open the possibility that the relationship is not strictly linear, and is also consistent with compressible (metallic) stripes throughout the doping range where they are observed. (See, e.g., Fig. 7 of Ref. 444.)

Most of the other experiments we have mentioned support the notion that the stripes are intrinsically metallic. Of course, the observed coexistence of static stripe order and superconductivity is a strong indicator of this, as presumably it would be hard to attribute long distance charge transport to stripe motion. ${ }^{33}$ The situation is most dramatic in nonsuperconducting LSCO with $0.02<x<0.05$, where the stripes are ordered [167,482], and far enough separated that the intrinsic properties of an individual stripe must surely determine the electronic structure - the mean stripe spacing [482] grows to be as large as $350 \AA$ or so for $x=0.02$. These materials exhibit 98, 483 a metallic (linear in $T$ ) temperature dependence of the resistivity down to moderate temperatures. More remarkably, as shown 4183] by Ando et al., although the magnitude of the resistance is large compared to the quantum of resistance at all temperatures, when interpreted in terms of a model in which the conduction occurs along dilute, metallic stripes, the inferred electron mobility within a stripe is nearly the same as that observed in optimally doped LSCO!

The Uemura plot and the Yamada plot may be about the same physics.
Are stripes good or bad for superconductivity? Striking empirical evidence which suggests that stripes and superconductivity are related comes from the Yamada plot [444], which reports $T_{c} v s$. the incommensurability seen in neutron scattering, i.e. the inverse spacing between stripes. First noted in LSCO, the relationship is remarkably linear for the underdoped region of the lanthanum compounds 444]. For far separated stripes, the transition temperature is depressed. As the stripes move closer together, and the Josephson coupling between them increases, $T_{c}$ increases. In addition, the similarities between the Yamada plot and the Uemura plot [107], which shows a linear relationship between $T_{c}$ and the superfluid density, indirectly imply that the Josephson coupling between stripes plays an important role in determining the macroscopic superfluid density.

It has been argued that since stripes compete with superconductivity, they cannot be involved in the mechanism of superconductivity [51]. (We would point out that, at the very least, such competition must imply that stripes and superconductivity are strongly connected.) The empirics are presently unclear on the issue. There is some evidence that static stripes compete with superconductivity, whereas fluctuating stripes enhance it. In instances where stripes are pinned, $T_{c}$ is generally suppressed, such as with $\mathrm{Nd}$ doping, $\mathrm{Zn}$

\footnotetext{
53 One could envisage stripe defect motion which transports charge perpendicular to the stripes, 481 but certainly the effective number of carriers due to this effect must be small.

54 This is equivalent to 64 (orthorhombic) lattice constants, $b_{\text {ortho }}^{*}=5.41 \AA$. 164
} 
doping, or at the $1 / 8$ anomaly. An exception to this trend occurs in the LCO family, which exhibits its highest $T_{c}$ for a static stripe ordered material. Recently, Ichikawa et al. [413] have argued that it is spin stripe order, rather than charge stripe order, which competes with superconductivity. Whatever the details, the gross trend in materials other than LCO seems to be that the highest transition temperatures are achieved for dopings that presumably do not support actual (static) stripe order. It is also worth noting that in LSCO 484 and YBCO 146], neutron scattering shows a gap developing in the incommensurate magnetic fluctuations at $T_{c}$, perhaps indicating that superconductivity favors fluctuating stripes.

On the other hand, $T_{c}$ is a nonmonotonic function of $x$, and pretty clearly determined by the lesser of two distinct energy scales. But the superconducting gap, as deduced from low temperature tunnelling or ARPES experiments deep in the superconducting state, is a monotonically decreasing function of $x$. It is generally believed that stripe correlations are similarly strongest when $x$ is small and vanish with sufficient overdoping, although in truth the direct experimental evidence for this intuitively obvious statement is not strong. Thus, there is at least a generally positive correlation between the degree of local stripe order and the most obvious scale characterizing pairing. This leads us to our next question:

Do stripes produce pairing? It is well known that the physics of an antiferromagnet is kinetic energy driven, and phase coherence must be kinetic energy driven when $T_{\text {pair }}>>T_{c}$, since spatial fluctuations of the phase drive pair currents. But can pair formation be kinetic energy driven? In particular, do stripes produce pairing? As reviewed in Section 11, numerical studies do find pairing in "fat" $1 \mathrm{D}$ systems.

However, there is no experiment we can point to that proves the pairing is either kinetic energy driven 55 or due to stripes. Nor is it clear what such an experiment would be. There are ways to falsify the conjecture that stripes produce pairing, such as a demonstration that stripes are not in some sense

55 The brilliantly conceived high precision measurements of the optical conductivity of van der Marel and collaborators 27, and more recently by Bontemps and collaborators [28], are highly suggestive in this regard. In optimally doped BSCCO, they observe a strongly temperature dependent change in the optical spectral weight integrated up to frequencies two orders of magnitude greater than $T_{c}-$ if interpreted in terms of the single band sum rule, this observation implies a decrease in the kinetic energy upon entering the superconducting state of a magnitude comparable to reasonable estimates of the condensation energy. This is very striking, since in a BCS superconductor, the kinetic energy would increase by precisely this amount. However, neither the single band sum rule, nor the notion of a condensation energy are unambiguously applicable in the present problem. This is the best existing evidence that the mechanism of superconductivity is kinetic energy driven, but it is not yet evidence that would stand up in court. 
ubiquitous in the cuprates, or a demonstration that pairing generally precedes local charge stripe formation as the temperature is lowered. We have discussed many predictions which find some support in experiments, such as the fact that static stripes are good for pairing but bad for phase coherence, and vice versa, and the systematics of the superconducting coherence peak. But these interpretations are not necessarily unique. Much of the phenomenology is consistent with a spin gap proximity effect mechanism of pairing, but we see no smoking gun.

Do stripes really make the electronic structure quasi-1D? Does the existence of stripes provide a sufficient excuse to treat the cuprates as selforganized quasi-1D conductors? If so, then we can apply many of the insights we have obtained directly, and without apology to the interpretation of experiment. As has been discussed in previous sections, and in considerably more detail in other places [6, 20, 86, 149, 471, 472, 474], there are many striking experiments in the cuprates that can be simply and naturally understood in this way. But do they actually affect the electronic structure so profoundly as to render it quasi-1D?

The most direct evidence comes from the ARPES results of Shen and collaborators [87] on the stripe ordered material, $\mathrm{La}_{1.6-x} \mathrm{Nd}_{0.4} \mathrm{Sr}_{x} \mathrm{CuO}_{4}$. These experiments reveal a remarkable confinement of the majority of the electronic spectral weight inside a dramatically 1D Fermi surface. This experiment probes fairly high energy excitations, and so demonstrates a profound effect of an ordered stripe array on all aspects of the electronic structure. More generally, studies have shown 86, 149, 471, 474 that many of the most striking features of the ARPES spectra of the cuprates are readily rationalized on the basis of an assumed, locally quasi-1D electronic structure.

Transport measurements are macroscopic, so even if locally the electronic structure is strongly quasi-1D, the effects of stripe meandering, domain formation, and disorder will always produce a substantially reduced effective anisotropy at long distances. From this perspective, the order 1, strongly temperature dependent transport anisotropies observed by Ando and collaborators [98] in LSCO and YBCO provide tangible evidence of a strong susceptibility of the electron liquid in the copper oxide planes to develop anisotropies in tensor response functions. Less direct, but even more dramatic evidence that stripes make the electron dynamics quasi-1D has been adduced from Hall effect measurements on the stripe ordered material, $\mathrm{La}_{1.6-x} \mathrm{Nd}_{0.4} \mathrm{Sr}_{x} \mathrm{CuO}_{4}$, by Noda et al. [485] They have observed that the Hall coefficient, $R_{H}$, which is relatively weakly temperature dependent above the stripe ordering transition temperature, $T_{c o}$, drops dramatically for $T<T_{c o}$, such that $R_{H} \rightarrow 0$ as $T \rightarrow 0$ for doped hole concentration, $x \leq 1 / 8$, and $R_{H}$ tends to a reduced but finite value for $x>1 / 8$. This observation was initially interpreted 485] as evidence that ordered stripes prevent coherent transverse motion of electrons within the copper oxide plane; this interpretation was later shown to be not 
entirely correct [207], although the basic conclusion that the stripes render the electron dynamics quasi-one dimensional is probably sound. Further evidence that stripe formation inhibits transverse electronic motion is strongly suggested by the observed suppression of c-axis coherent charge motion in the stripe ordered state of the same materials [486].

However, it would be very desirable to develop new strategies to directly address this issue. For instance, a defect, such as a twin bounary, could purposely be introduced to locally aline the stripe orientation, and the induced electronic anisotropy then be detected with STM.

What about overdoping? On the underdoped side of the phase diagram of the cuprates, the energy scales of $T_{\text {stripe }}^{*}, T_{\text {pair }}^{*}$, and $T_{c}$ are generally sufficiently separated to make the application of many of these ideas plausible. Yet on the overdoped side, the energy scales seem to come crashing into each other, depressing $T_{c}$. Furthermore, on the overdoped side, we have $T_{\theta}>T_{\text {pair }}$, in violation of a common assumption we have made throughout this article. The very existence of stripes on the overdoped side is questionable. The Uemura and Yamada plot are not satisfied there. If there are no stripes, and yet there is superconductivity, this does not bode well for a stripes based mechanism. Indeed, it is easier to believe that a mean field like solution is crudely applicable on the overdoped side, where $T_{c}$ is closer to $T_{\text {pair }}$ than it is to $T_{\theta}$.

One possibility is that the superconducting state far on the overdoped side (especially, where $T_{c}$ is low and the normal state ARPES spectrum begins to look more Fermi liquid-like) is best approached in terms of a Fermi surface instability and a BCS-Eliashberg mechanism, while on the underdoped side it is best viewed from a stripes perspective. In keeping with the multiscale approach advocated above, it may be no simple matter to unify these approaches in a smooth way.

However, there is an attractive possibility that is worth mentioning here. As we have mentioned, in a stripe liquid, so long as the characteristic stripe fluctuations frequency, $\hbar \bar{\omega}$, is small compared to the superconducting gap scale, the stripes can be treated as quasi-static for the purposes of understanding the mechanism of pairing. Conversely, when $\hbar \bar{\omega} \gg \Delta_{0}$, the stripe fluctuations can be integrated out to yield an effectively homogeneous system with an induced interaction between electrons. Indeed, it has previously been proposed [125] that stripe fluctuations themselves are a candidate for the "glue" that mediates an effective attraction between electrons. It is easy to imagine that $\hbar \bar{\omega} / \Delta_{0}$ is a strongly increasing function of $x$. A sort of unification of the two limits could be achieved if stripe fluctuations play the role of the intermediate boson which mediates the pairing in highly overdoped materials, while in underdoped and optimally doped materials the system can be broken up into quasi-1D ladders, which exhibit the spin gap proximity effect. 
How large is the regime of substantial fluctuation superconductivity? This important question is fundamentally ill-defined. It is important, because its answer determines the point of view we take with regard to a number of key experiments. But it is ill-defined in the following sense: in the neighborhood of any phase transition, there is a region above $T_{c}$ where substantial local order exists, but how broad the fluctuation region is said to be depends on exactly how "substantial" is defined, or measured. There has been an enormous amount written on this subject already, so we will just make a few brief observations.

Because in one dimension, phase fluctuations always reduce the superconducting $T_{c}$ to zero, in a quasi-one dimensional superconductor (i.e. in the limit of large anisotropy), there is necessarily a parametrically large fluctuation regime between the mean field transition temperature and the actual ordering temperature.

The finite frequency superfluid density measured in BSCCO 170 with $T_{c}=74 K$ shows a local superfluid density persists up to at least $90 K$, indicative of fluctuation superconductivity in that regime. Both microwave absorption [171 and thermal expansivity measurements 169] on optimally doped YBCO detect significant critical superconducting fluctuations within $\pm 10 \%$ of $T_{c}$. All of these experiments are well accounted for in terms of the critical properties of a phase-only (XY) model, and are not well described as Gaussian fluctuations of a Landau-Ginzberg theory. Thus, there is no question that there is a well defined magnitude of the order parameter, and substantial local superconducting order for at least $10 \mathrm{~K}$ to $20 \mathrm{~K}$ above $T_{c}$, and a correspondingly broad range of substantial phase fluctuations below $T_{c}$.

There are, however, some experiments that suggest that substantial local pairing persists in a much broader range of temperatures. [487] Nernst measurements [488,489 have detected vortex-like signals up to $100 \mathrm{~K}$ above $T_{c}$ in $\mathrm{LSCO}$ and $\mathrm{YBCO}$, i.e. to temperatures up to 5 times $T_{c}$ ! In both cases, however, the final word has yet to be spoken concerning the proper interpretation of these intriguing experiments. 490] ARPES 96, 491 and tunnelling [97] studies find that the gap in BSCCO persists up to $100 \mathrm{~K}$ above $T_{c}$, i.e. to temperatures of order two or more times $T_{c}$.

Finally, there are preliminary indications that there may be substantial local superconducting order in severely underdoped materials in which no macroscopic indications of superconductivity appear at any temperature. Presumably, if this is the case, long range phase coherence has been suppressed in these materials by quantum phase fluctuations 287 which proliferate due to the small bare superfluid stiffness and the poor screening of the Coulomb potential. In particular, experiments on films of severely underdoped nonsuperconducting YBCO have revealed that a metastable superconducting state can be induced by photodoping. This has permitted the patterning of small scale superconducting structures, in which it has been shown 492 that substantial Josephson coupling between two superconduct- 
ing regions can persist even when they are separated by as much as $1000 \AA$. This "anomalous proximity effect" implies that there is a substantial pair field susceptibility in this nonsuperconducting material.

What about phonons? Phonons are clearly strongly coupled to the electron gas in the cuprates. Certainly, when there is charge order of any sort, it is unavoidable that it induces (or is induced by) lattice distortions. Manifestly, phonons will enhance any electronic tendency to phase separation or stripe formation 124. They will also tend to make any stripes "heavy," and so suppress quantum fluctuations - likely, this leads to a depression of superconductivity. There is a dramatic isotope effect anomaly seen [493] in some materials when the doped hole density, $x=1 / 8$; presumably, this is related to just such a phonon-induced pinning of the stripe order 426 . Recently, there has been considerable controversy generated by the suggestion 428 that certain features of the ARPES spectrum of a wide class of cuprates reflect the effects of strong electron-phonon coupling. This is clearly an area in which much work remains to be done. In our opinion, other than in $1 \mathrm{D}$, the effects of electron-phonon coupling in a strongly correlated electron gas is an entirely unsolved problem.

What are the effects of quenched disorder? We have said essentially nothing about the effects of quenched disorder on the materials of interest, although the materials are complicated, and disorder is always present. There are even some theories which consider the disorder to be essential to the mechanism of high temperature superconductivity [494]. A strong case against this proposition is made by the observation that as increasingly well ordered materials are produced, including some which are stoichiometric and so do not have any of the intrinsic disorder associated with a random alloy, the superconducting properties are not fundamentally altered, and that if anything $T_{c}$ and the superfluid density both seem to rise very slightly as disorder is decreased.

However, other properties of the system are manifestly sensitive to disorder. Since disorder couples to spatial symmetry breaking order parameters in the same way that a random field couples to a magnetic order parameter, it is generally a relevant perturbation. Among other things, this means that none of the stripe orders discussed above will ever occur as true long range order, and the putative transitions are rounded and rendered glassy [414, 495 497. So even the supposedly sharp statements discussed above are only sharp, in practice, if we can study such highly perfect crystals that they approximate the disorder $\rightarrow 0$ limit. This is a general problem. Progress has been made in recent years in growing more and more perfect single crystals of particular stoichiometric superconductors. Clearly, advances in this area are an essential component of the ongoing effort to unravel the physics of these materials.

This is a good question. 


\section{Acknowledgements}

We would especially like to acknowledge the profound influence on our understanding of every aspect of the physics discussed in this paper of discussions with our colleagues and collaborators, John Tranquada, Vadim Oganesyan, and Eduardo Fradkin. We also want to explicitly aknowledge the extremely helpful suggestions and critiques we obtained from J. W. Allen, Y. Ando, N. P. Armitage, A. Auerbach, D. A. Bonn, R. J. Birgeneau, E. Dagotto, A. H. Castro Neto, E. Fradkin, S. Sachdev, D. J. Scalapino, S. L. Sondhi, J. M. Tranquada, and O. Zachar. Finally, we have benefited greatly from discussion of the ideas presented herein with more colleagues than we can hope to acknowledge, but we feel we must at least acknowledge our intellectual debts to G. Aeppli, J. W. Allen, P. B. Allen, Y. Ando, D. N. Basov, M. R. Beasley, A. H. Castro Neto, S. Chakravarty, E. Daggatto, P. C. Dai, J. C. S. Davis, C. Di Castro, R. C. Dynes, H. Eisaki, A. Finkelstein, M. P. A. Fisher, T. H. Geballe, M. Granath, P. D. Johnson, C. Kallin, A. Kapitulnik, H.Y. Kee, Y.-B. Kim, R. B. Laughlin, D-H. Lee, Y. S. Lee, K. A. Moller, H. A. Mook, C. Nayak, Z. Nussinov, N. P. Ong, J. Orenstein, S. Sachdev, D. J. Scalapino, J. R. Schrieffer, Z-X. Shen, S. L. Sondhi, B. I. Spivak, T. Timusk, T. Valla, J. Zaanen, O. Zachar, S-C. Zhang, and X. J. Zhou. This work was supported, in part, by NSF grant DMR-0110329 at UCLA, DOE grant DE-FG03-00ER45798 at UCLA and BNL, and NSF grant DMR97-12765 and the Office of the Provost at Boston University. 


\section{List of Symbols}

\begin{tabular}{|c|c|}
\hline Symbol & Definition, page \\
\hline$A^{<}(\boldsymbol{k}, \omega)$ & Single hole spectral function, 40 \\
\hline CDW & Charge density wave, 46 \\
\hline$d$ & Dimension, 20 \\
\hline$D(l)$ & Correlation function of the pair field, 99 \\
\hline$D_{\alpha}$ & Scaling dimension of $O_{\alpha}, 46$ \\
\hline$E_{F}$ & Fermi energy, 7 \\
\hline$E_{s}$ & Spin spectrum, 38 \\
\hline$F_{\eta, \sigma}$ & Klein factor, 34 \\
\hline G & Reciprocal lattice vector, 33 \\
\hline$g_{1}$ & Backscattering, 33 \\
\hline$g_{1 \|}$ & Backscattering of same spin particles, 33 \\
\hline$g_{1 \perp}$ & Backscattering of opposite spin particles, 33 \\
\hline$g_{2}$ & Forward scattering on both branches, 33 \\
\hline$g_{3}$ & Umklapp scattering, 33 \\
\hline$g_{4}$ & Forward scattering within same branch, 33 \\
\hline$G_{c}$ & Charge piece of the one hole spectral function, 42 \\
\hline$G_{s}$ & Spin piece of the one hole spectral function, 42 \\
\hline$h_{j}(x)$ & Transverse stripe displacement, 51 \\
\hline$J$ & Nearest neighbor exchange coupling, 97, 114 \\
\hline$k_{B}$ & Boltzmann's constant, 7 \\
\hline$K_{c}$ & Charge Luttinger parameter, 35 \\
\hline$k_{F}$ & Fermi wavevector, 33 \\
\hline$K_{s}$ & Spin Luttinger parameter, 35 \\
\hline$L_{j}(x)$ & Arc length, 51 \\
\hline$m^{*}$ & Effective pair mass, 12,60 \\
\hline$n_{2 d}$ & Density of doped holes per plane, 12 \\
\hline$n_{e}$ & Electron density, 105 \\
\hline$N\left(E_{F}\right)$ & Density of states at $E_{F}, 18$ \\
\hline$n_{s}$ & Superfluid density, 60 \\
\hline SDW & Spin density wave, 46 \\
\hline SS & Singlet superconductivity, 46 \\
\hline$S_{z}(x)$ & Spin density, 34 \\
\hline$T$ & Temperature, 23 \\
\hline $1 / T_{1} T$ & NMR relaxation rate, 22 \\
\hline
\end{tabular}


t

$T_{c}$

$T_{p}$

$t_{\perp}$

TS

$T_{\text {pair }}^{*}$

$T_{\text {stripe }}^{*}$

$T_{\theta}$

$U$

V

$v_{c}$

$v_{F}$

$V_{\boldsymbol{k}, \boldsymbol{k}^{\prime}}$

$v\left(k_{\perp}\right)$

$v_{s}$

$x$

$Z$

$Z_{s}$

$\Delta_{k}$

$\Delta_{0}$

$\Delta_{s}$

$\partial_{x} \theta_{c}$

$\partial_{x} \theta_{c}$

$\gamma$

$\gamma_{c}$

$\gamma_{s}$

$\kappa\left(k_{\perp}\right)$

$\lambda$

$\mu$

$\mu^{*}$

$\omega_{D}$

$\omega_{P}$

$\phi_{c}$

$\phi_{s}$

$\pi$

$\psi_{\eta, \sigma}^{\dagger}$

$\rho(x)$

$\varrho$

$\tau$
Nearest neighbor hopping, 97, 114

Superconducting transition temperature, 1

Pairing scale, 11

Interchain single particle tunnelling, 49

Triplet superconductivity, 46

Crossover temperature at which pairs form, 31

Crossover temperature at which charge stripes form, 31

Phase ordering scale, 12

On-site Hubbard interaction, 12

Nearest neighbor interaction, 114

Charge velocity, 35

Fermi velocity, 33

BCS pair potential, 21

Velocity at the smectic fixed point, 54

Spin velocity, 35

Hole doping, 16

Coherent quasiparticle weight, 48

Coherent spin soliton weight, 44

BCS gap parameter, 20

Superconducting gap maximum, 1

Spin gap, 30

Conjugate momentum of $\phi_{c}, 34$

Conjugate momentum of $\phi_{s}, 34$

Helicity modulus, 60

Charge Luttinger exponent, 42

Spin Luttinger exponent, 42

Luttinger parameter at the smectic fixed point, 54

Bare electron-phonon coupling, 11

Bare Coulomb repulsion, 11

Renormalized Coulomb repulsion, 11

Debye frequency, 7, 25, 40, 74

Plasma frequency, 25

Bosonic charge field, 34

Bosonic spin field, 34

3.141592653589793238462643. ., 18

Fermion creation operator in the 1DEG, 33

Charge density, 34

Hole density on a stripe, 105

Quasiparticle lifetime, 40 


\section{References}

1. J. R. Schrieffer, Theory of Superconductivity, Frontiers in Physics (AddisonWesley) (1988).

2. J. G. Bednorz and K. A. Muller, "Possible high $\mathrm{T}_{c}$ superconductivity in the Ba-La-Cu-O system," Z. Phys. B, 64, 189-193 (1986).

3. V. J. Emery and S. A. Kivelson, "Superconductivity in bad metals," Phys. Rev. Lett., 74, 3253-3256 (1995).

4. P. W. Anderson, "Experimental constraints on the theory of high-Tc superconductivity," Science, 256, 1526-1531 (1992).

5. P. W. Anderson, "The resonating valence bond state in $\mathrm{La}_{2} \mathrm{CuO}_{4}$ and superconductivity," Science, 235, 1169-1198 (1987).

6. V. J. Emery, S. A. Kivelson, and J. M. Tranquada, "Stripe phases in hightemperature superconductors," Proc. Natl. Acad. Sci., 96, 8814-8817 (1999).

7. P. A. Lee, "Pseudogaps in underdoped cuprates," Physica C, 317-318, 194204 (1999).

8. L. Taillefer, B. Lussier, R. Gagnon, K. Behnia, and H. Aubin, "Universal heat conduction in $\mathrm{YBa}_{2} \mathrm{Cu}_{3} \mathrm{O}_{6.9}$," Phys. Rev. Lett., 79, 483-486 (1997).

9. P. J. Turner, R. Harris, S. Kamal, M. E. Hayden, D. M. Broun, D. C. Morgan, A. Hosseini, P. Dosanjh, J. S. Preston, R. Liang, D. A. Bonn, and W. N. Hardy, "Broadband microwave spectroscopy of d-Wave quasiparticles in Oxygenordered $\mathrm{YBa}_{2} \mathrm{Cu}_{3} \mathrm{O}_{6.50}$," cond-mat/0111353, submitted to PRL (2002).

10. K. A. Moller, D. J. Baar, J. S. Urbach, R. Liang, W. N. Hardy, and A. Kapitulnik, "Magnetic field dependence of the density of states of $\mathrm{YBa}_{2} \mathrm{Cu}_{3} \mathrm{O}_{6.95}$ as determined from the specific heat," Phys. Rev. Lett., 73, 2744-2747 (1994).

11. B. Revaz, J.-Y. Genoud, J. K. Neumaier, A. Erb, and E. Walker, " $d$-wave scaling relations in the mixed-state specific heat of $\mathrm{YBa}_{2} \mathrm{Cu}_{3} \mathrm{O}_{7}, "$ Phys. Rev. Lett., 80, 3364-3368 (1998).

12. D. H. Lu, D. L. Feng, N. P. Armitage, K. M. Shen, A. Damascelli, C. Kim, F. Ronning, Z. X. Shen, D. A. Bonn, R. Liang, W. N. Hardy, A. I. Rykov, and $\mathrm{S}$. Tajima, "Superconducting gap and strong in-plane anisotropy in untwinned $\mathrm{YBa}_{2} \mathrm{Cu}_{3} \mathrm{O}_{7-\delta}$," Phys. Rev. Lett., 86, 4370-4373 (2001).

13. D. Basov, R. Liang, D. A. Bonn, W. N. Hardy, B. Dabrowski, D. B. T. M. Quijada, J. P. Rice, D. M. Ginsberg, and T. Timusk, "In-plane anisotropy of the penetration depth in $\mathrm{YBa}_{2} \mathrm{Cu}_{3} \mathrm{O}_{7-x}$ and $\mathrm{YBa}_{2} \mathrm{Cu}_{4} \mathrm{O}_{8}$," Phys. Rev. Lett., 74, 598-601 (1995).

14. S. Chakravarty and S. Kivelson, "Electronic mechanism of superconductivity in the cuprates, C60, and polyacenes," Phys. Rev. B, 64, 064511-064519 (2001).

15. V. J. Emery and S. A. Kivelson, "Frustrated electronic phase separation and high-temperature superconductors," Physica C, 209, 597-621 (1993). 
16. S. Caprara, C. Castellani, C. Di Castro, and M. Grilli, "Phase separation and superconductivity in strongly interacting electron systems," Physica $C$, 235-240, 2155-2156 (1994).

17. A. Moreo, S. Yunoki, and E. Dagotto, "Phase separation scenario for manganese oxides and related materials," Science, 283, 2034-2040 (1999).

18. P. W. Anderson, "A re-examination of concepts in magnetic metals: the 'nearly antiferromagnetic Fermi liquid"," Adv. Phys., 46, 3-11 (1997).

19. J. E. Hirsch, "Antiferromagnetism, localization, and pairing in a twodimensional model for CuO2," Phys. Rev. Lett., 59, 228-231 (1987).

20. V. J. Emery, S. A. Kivelson, and O. Zachar, "Spin-gap proximity effect mechanism of high-temperature superconductivity," Phys. Rev. B, 56, 6120-6147 (1997).

21. S. Chakravarty, A. Sudbo, P. W. Anderson, and S. Strong, "Interlayer tunneling and gap anisotropy in high-temperature superconductors," Science, 261, 337-340 (1993).

22. E. Demler and S.-C. Zhang, "Quantitative test of a microscopic mechanism of high-temperature superconductivity," Nature, 396, 733-737 (1998).

23. A. H. Castro Neto and F. Guinea, "Superconductivity, Josephson coupling, and order parameter symmetry in striped cuprates," Phys. Rev. Lett., 80, 4040-4043 (1998).

24. J. E. Hirsch, "The true colors of cuprates," Science, 295, 2226-2227 (2002).

25. V. J. Emery, S. A. Kivelson, and O. Zachar, "Classification and stability of phases of the multicomponent one-dimensional electron gas," Phys. Rev. B, 59, 15641-15653 (1999).

26. D. J. Scalapino, "The 2-leg Hubbard ladder: Computational studies of new materials," cond-mat/0109125 (2001).

27. H. J. A. Molegraaf, C. Presura, D. van der Marel, P. H. Kes, and M. Li, "Superconductivity-induced transfer of in-plane spectral weight in $\mathrm{Bi}_{2} \mathrm{Sr}_{2} \mathrm{CaCu}_{2} \mathrm{O}_{8+\delta}$," Science, 295, 2239-2241 (2002).

28. A. F. Santander-Syro, R. P. S. M. Lobo, N. Bontemps, Z. Konstantinovic, Z. Z. Li, and H. Raffy, "Pairing in cuprates from high energy electronic states," cond-mat/0111539 (2001).

29. P. Monthoux, A. V. Balatsky, and D. Pines, "Toward a theory of hightemperature superconductivity in the antiferromagnetically correlated cuprate oxides," Phys. Rev. Lett., 67, 3448-3451 (1991).

30. N. Bulut and D. J. Scalapino, "d $\left(\mathrm{x}^{2}-y^{2}\right)$ symmetry and the pairing mechanism," Phys. Rev. B, 54, 14971-14973 (1996).

31. D. J. Scalapino, "Superconductivity and spin fluctuations," J. Low Temp. Phys., 117, 179-188 (1999), international Conference on Physics and Chemistry of Molecular and Oxide Superconductors. MOS'99, Stockholm, Sweden, 28 July-2 Aug. 1999. Kluwer Academic/Plenum Publishers.

32. R. J. Radtke, S. Ullah, K. Levin, and N. R. Norman, "Constraints on superconducting transition temperatures in the cuprates: antiferromagnetic spin fluctuations," Phys. Rev. B, 46, 11975-11985 (1992).

33. C. M. Varma, J. Zaanen, and K. Raghavachari, "Superconductivity in the fullerenes," Science, 254, 989-992 (1991).

34. M. Schluter, M. Lannoo, M. Needels, G. A. Baraff, and D. Tomanek, "Electron-phonon coupling and superconductivity in alkali-intercalated C60 solid," Phys. Rev. Lett., 68, 526-529 (1992). 
35. P. Morel and P. W. Anderson, "Calculation of the superconducting state parameters with retarded electron-phonon interaction," Physical Review, 125, 1263-1271 (1962).

36. J. R. Schrieffer, D. J. Scalapino, and J. W. Wilkins, "Effective tunneling density of states in superconductors," Phys. Rev. Lett., 10 (1963).

37. S. Chakravarty, S. Khlebnikov, and S. Kivelson, "Comment on "Electronphonon coupling and superconductivity in alkali-intercalated C60 solid"," Phys. Rev. Lett., 69, 212 (1992).

38. R. Shankar, "Renormalization-group approach to interacting fermions," Rev. Mod. Phys., 66, 129-192 (1994).

39. J. Polchinski, "Renormalization and effective lagrangians," Nucl. Phys. B, 231, 269-295 (1984).

40. Z.-X. Shen, D. S. Dessau, B. O. Wells, D. M. King, W. E. Spicer, A. J. Arko, D. Marshal, L. W. Lombardo, A. Kapitulnik, P. Dickinson, S. Doniach, J. DiCarlo, A. G. Losser, and C. H. Park, "Anomalously large gap anisotropy in the $a-b$ plane of bi2212," Phys. Rev. Lett., 70, 1553-1556 (1993).

41. R. Micnas, J. Ranninger, and S. Robaszkiewicz, "Superconductivity in narrowband systems with local nonretarded attractive interactions," Rev. Mod. Phys., 62, 113-234 (1990).

42. T. Holstein, "Studies of polaron motion: I," Annals of Physics., 8, 325-342 (1959).

43. G. Gruner, Density waves in solids (Perseus Books Group) (2000).

44. G. Bilbro and W. L. McMillan, "Theoretical model of superconductivity and the martensitic transformation in A15 compounds," Phys. Rev. B, 14, 1887$1892(1976)$.

45. O. Zachar, S. A. Kivelson, and V. J. Emery, "Landau theory of stripe phases in cuprates and nickelates," Phys. Rev. B, 57, 1422-1426 (1998).

46. J. M. Tranquada, "Phase separation, charge segregation and superconductivity in layered cuprates," Neutron Scattering in Layered Copper-Oxide Superconductors, A. Furrer, Editor, 83, 225-260 (1998), kluwer, Dordrecht, The Netherlands.

47. J. M. Tranquada, "Experimental evidence for topological doping in the cuprates," AIP-Conference-Proceedings, 483, 336-340 (1999).

48. J. Zaanen, "High-temperature superconductivity: stripes defeat the Fermi liquid," Nature, 404, 714 (2000).

49. S. Sachdev, "Quantum criticality: competing ground states in low dimensions," Science, 288, 475-80 (2000).

50. J. Orenstein and A. J. Millis, "Advances in the physics of high-temperature superconductivity," Science, 288, 468-474 (2000).

51. G. Baskaran, "Competition between superconductivity and charge stripe order in high-Tc cuprates," Mod. Phys. Lett. B, 14, 377-384 (2000).

52. S. A. Kivelson, E. Fradkin, and V. J. Emery, "Electronic liquid-crystal phases of a doped Mott insulator," Nature, 393, 550-553 (1998).

53. C. M. Varma, "Non Fermi-liquid states and pairing of a general model of copper-oxide metals," Phys. Rev. B, 55, 14554-14580 (1997).

54. S. Chakravarty, R. B. Laughlin, D. K. Morr, and C. Nayak, "Hidden order in the cuprates," Phys. Rev. B, 63, 094503-094510 (2001).

55. I. Affleck and J. B. Marston, "Large-n limit of the Heisenberg-Hubbard model: Implications for high- $\mathrm{T}_{c}$ superconductors," Phys. Rev. B, 37, 3774-3777 (1988). 
56. G. Kotliar, "Resonating valence bonds and d-wave superconductivity," Phys. Rev. B, 37, 3664-3666 (1998).

57. D. A. Ivanov, P. A. Lee, and X.-G. Wen, "Staggered-vorticity correlations in a lightly doped t-J model: A variational approach," Phys. Rev. Lett., 34, 3958-3961 (2000).

58. Q. H. Wang, J. H. Han, and D. H. Lee, "Staggered currents in the mixed state," Phys. Rev. Lett., 87, 7004-7007 (2001).

59. T. Senthil and M. P. A. Fisher, "Fractionalization in the cuprates: Detecting the topological order," Phys. Rev. Lett., 86, 292-295 (2000).

60. S. A. Kivelson, G. Aeppli, and V. J. Emery, "Thermodynamics of the interplay between magnetism and high-temperature superconductivity," Proc. Nat. Acad. Sci., 98, 11903-11907 (2001).

61. C. Castellani, C. Di Castro, and M. Grilli, "Singular quasiparticle scattering in the proximity of charge instabilities," Phys. Rev. Lett., 75, 4650-4653 (1995).

62. S. Andergassen, S. Caprara, C. Di Castro, and M. Grilli, "Anomalous isotopic effect near the charge-ordering quantum criticality," Phys. Rev. Lett., 87, 56401-56403 (2001).

63. A. V. Chubukov, S. Sachdev, and J. Ye, "Theory of two-dimensional quantum Heisenberg antiferromagnets with a nearly critical ground state," Phys. Rev. $B, 49,11919-11961$ (1994).

64. N. D. Mathur, F. M. Grosche, S. R. Julian, I. R. Walker, D. M. Freye, R. K. W. Haselwimmer, and G. G. Lonzarich, "Magnetically mediated superconductivity in heavy fermion compounds," Nature, 394, 39-43 (1998).

65. S. A. Grigera, R. S. Perry, A. J. Schofield, M. Chiao, S. R. Julian, G. G. Lonzarich, S. I. Ikeda, Y. Maeno, A. J. Millis, and A. P. Mackenzie, "Magnetic field-tuned quantum criticality in the metallic ruthenate $\mathrm{Sr}_{3} \mathrm{Ru}_{2} \mathrm{O}_{7}$," Science, 294, 329-332 (2001).

66. J. R. Schrieffer, "Ward's identity and the suppression of spin fluctuation superconductivity," J. Low Temp. Phys., 99, 397-402 (1995).

67. R. B. Laughlin, "A critique of two metals," Adv. Phys., 47, 943-958 (1998).

68. G. Baskaran, Z. Zou, and R. B. Laughlin, "The resonating valence bond state and high $\mathrm{T}_{c}$ superconductivity - a mean field theory," Solid State Comm., 63, 973-976 (1987).

69. S. A. Kivelson, D. S. Rokhsar, and J. P. Sethna, "Topology of the resonating valence-bond state: Solitons and high- $\mathrm{T}_{c}$ superconductivity," Phys. Rev. B, 35, 8865-8868 (1987).

70. D. S. Rokhsar and S. A. Kivelson, "Superconductivity and the quantum hardcore dimer gas," Phys. Rev. Lett., 61, 2376-2379 (1988).

71. N. Read and S. Sachdev, "Large-N expansion for frustrated quantum antiferromagnets," Phys. Rev. Lett., 66, 1773-1776 (1991).

72. V. Kalmeyer and R. B. Laughlin, "Theory of the spin liquid state of the Heisenberg antiferromagnet," Phys. Rev. B, 39, 11879-11899 (1989).

73. X. G. Wen, F. Wilcek, and A. Zee, "Chiral spin states and superconductivity," Phys. Rev. B, 39, 11413-11423 (1989).

74. P. B. Wiegmann, "Superconductivity in strongly correlated electronic systems and confinement versus deconfinement phenomenon," Phys. Rev. Lett., 60, 821-824 (1988).

75. L. Balents, M. P. A. Fisher, and C. Nayak, "Nodal liquid theory of the pseudo-

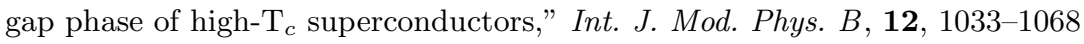
(1998). 
76. L. Balents, M. P. A. Fisher, and C. Nayak, "Dual order parameter for the nodal liquid," Phys. Rev. B, 60, 1654-1667 (1999).

77. T. Senthil and M. P. A. Fisher, "Z2 gauge theory of electron fractionalization in strongly correlated systems," Phys. Rev. B, 62, 7850-7881 (2000).

78. X. G. Wen, "Topological orders in rigid states," Int. J. Mod. Phys. B, 4, 239-271 (1990).

79. R. Moesner and S. L. Sondhi, "Resonating valence bond phase in the triangular lattice quantum dimer model," Phys. Rev. Lett., 86, 1881-1884 (2001).

80. R. Moessner, S. L. Sondhi, and E. Fradkin, "Short-ranged resonating valance bond physics, quantum dimer models, and Ising gauge theories," Phys. Rev. $B, \mathbf{6 5}, 024504-024516(2002)$.

81. T. Timusk and B. Statt, "The pseudogap in high-temperature superconductors: an experimental survey," Rep. Prog. Phys., 62, 61-122 (1999).

82. J. L. Tallon and J. W. Loram, "The doping dependence of $T^{*}$ - what is the real high $T_{c}$ phase diagram?" Physica $C$, 349, 53-68 (2001).

83. A. Leggett, "Cuprate Superconductivity: Dependence of $\mathrm{T}_{c}$ on the c-Axis Layering Structure," Phys. Rev. Lett., 83, 392-395 (1999).

84. P. W. Anderson, The Theory of Superconductivity in the Cuprates (Princeton University Press, Princeton, NJ) (1997).

85. R. B. Laughlin, "Evidence for quasiparticle decay in photoemission from underdoped cuprates," Phys. Rev. Lett., 79, 1726-1729 (1997).

86. D. Orgad, S. A. Kivelson, E. W. Carlson, V. J. Emery, X. J. Zhou, and Z. X. Shen, "Evidence of electron fractionalization from photoemission spectra in the high temperature superconductors," Phys. Rev. Lett., 86, 4362-4365 (2001).

87. X. J. Zhou, P. Bogdanov, S. A. Kellar, T. Noda, H. Eisaki, S. Uchida, Z. Hussain, and Z.-X. Shen, "One-dimensional electronic structure and suppression of d-Wave node state in $\mathrm{La}_{1.28} \mathrm{Nd}_{0.6} \mathrm{Sr}_{0.12} \mathrm{CuO}_{4}, "$ Science, 286, 268-272 (1999).

88. T. Valla, A. V. Fedorov, P. D. Johnson, Q. Li, G. D. Gu, and N. Koshizuka, "Temperature dependent scattering rates at the Fermi surface of optimally doped $\mathrm{Bi}_{2} \mathrm{Sr}_{2} \mathrm{CaCu}_{2} \mathrm{O}_{8+\delta}$," Phys. Rev. Lett., 85, 828-831 (2000).

89. A. V. Fedorov, T. Valla, P. D. Johnson, Q. Li, G. D. Gu, and N. Koshizuka, "Temperature dependent photoemission studies of optimally doped $\mathrm{Bi}_{2} \mathrm{Sr}_{2} \mathrm{CaCu}_{2} \mathrm{O}_{8}$," Phys. Rev. Lett., 82, 2179-2182 (1999).

90. D. L. Feng, D. H. Lu, K. M. Shen, S. Oh, A. Andrus, J. O'Donnell, J. N. Eckstein, J. Shimoyama, K. Kishio, and Z. X. Shen, "On the similarity of the spectral weight pattern of $\mathrm{Bi}_{2} \mathrm{Sr}_{2} \mathrm{CaCuO}_{8+\delta}$ and $\mathrm{La}_{1.48} \mathrm{Nd}_{0.4} \mathrm{Sr}_{0.12} \mathrm{CuO}_{4}$," Physica C, 341, 2097-2098 (2000).

91. A. G. Loeser, Z.-X. Shen, M. C. Schabel, C. Kim, M. Zhang, A. Kapitulnik, and P. Fournier, "Temperature and doping dependence of the Bi-Sr-Ca-Cu-O electronic structure and fluctuation effects," Phys. Rev. B, 56, 14185-14189 (1997).

92. C. M. Varma, P. B. Littlewood, S. Schmittrink, E. Abrahams, and A. E. Ruckenstein, "Phenomenology of the normal state of the $\mathrm{Cu}-\mathrm{O}$ high-temperature superconductors," Phys. Rev. Lett., 63, 1996-1999 (1989).

93. C. M. Varma, Z. Nussinov, and W. van Saarloos, "Singular Fermi liquids," cond-mat/0103393 (2001).

94. E. Abrahams and C. M. Varma, "What angle-resolved photoemission experiments tell us about the microscopic theory for high-temperature superconductors," Proc. Natl. Accad. Sci. (2000). 
95. J. M. Harris, Z.-X. Shen, P. J. White, D. S. Marshall, M. C. Schabel, J. N. Eckstein, and I. Bozovic, "Anomalous superconducting state gap size versus Tc behavior in underdoped $\mathrm{Bi}_{2} \mathrm{Sr}_{2} \mathrm{Ca}_{1-x} \mathrm{Dy}_{x} \mathrm{Cu}_{2} \mathrm{O}_{8}, "$ Phys. Rev. B, 54, R15665-R15668 (1996).

96. H. Ding, T. Yokoya, J. C. Campuzano, T. Takahashi, M. Randeria, M. R. Norman, and T. M. K. H. J. Giapintzakis, "Spectroscopic evidence for a pseudogap in the normal state of underdoped high- $T_{c}$ superconductors," Nature, 382, 51-54 (1996).

97. C. Renner, B. Revaz, J.-Y. Genoud, K. Kadowaki, and O. Fischer, "Pseudogap precursor of the superconducting gap in under- and overdoped $\mathrm{Bi}_{2} \mathrm{Sr}_{2} \mathrm{CaCu}_{2} \mathrm{O}_{8+\delta}$," Phys. Rev. Lett., 80, 149-152 (1998).

98. Y. Ando, K. Segawa, S. Komiya, and A. N. Lavrov, "Electrical resistivity anisotropy from self-organized one-dimensionality in high-temperature superconductors," Phys. Rev. Lett., 88, 137005-137008 (2002).

99. I. Maggio-Aprile, C. Renner, A. Erb, E. Walker, and O. Fischer, "Direct vortex lattice imaging and tunneling spectroscopy of flux lines on $\mathrm{YBa}_{2} \mathrm{Cu}_{3} \mathrm{O}_{7-\delta}$," Phys. Rev. Lett., 75, 2754-2757 (1995).

100. C. Howald, P. Fournier, and A. Kapitulnik, "Inherent inhomogeneities in tunneling spectra of $\mathrm{Bi}_{2} \mathrm{Sr}_{2} \mathrm{CaCu}_{2} \mathrm{O}_{8-x}$ crystals in the superconducting state," Phys. Rev. B, 64, 100504-100507 (2001).

101. S. H. Pan, J. P. O’Neal, R. L. Badzey, C. Chamon, H. Ding, J. R. Engelbrecht, Z. Wang, H. Eisaki, S. Uchida, A. K. Guptak, K. W. Ng, E. W. Hudson, K. M. Lang, and J. C. Davis, "Microscopic electronic inhomogeneity in the high $T_{c}$ superconductor $\mathrm{Bi}_{2} \mathrm{Sr}_{2} \mathrm{CaCu}_{2} \mathrm{O}_{8+x}$," Nature, 413, 282-285 (2001).

102. J. E. Sonier, J. H. Brewer, R. F. Kiefl, D. A. Bonn, S. R. Dunsiger, W. N. Hardy, R. Liang, W. A. MacFarlane, R. I. Miller, and T. M. Riseman, "Measurements of the fundamental length scales in the vortex state of $\mathrm{YBa}_{2} \mathrm{Cu}_{3} \mathrm{O}_{6.60}$," Phys. Rev. Lett., 79, 2875-2878 (1997).

103. C. A. R. S. de Melo, M. Randeria, and J. R. Engelbrecht, "Crossover from BCS to Bose superconductivity: Transition temperature and time-dependent Ginzburg-Landau theory," Phys. Rev. Lett., 71, 3202-3205 (1993).

104. Q. Chen, I. Kosztin, B. Janko, and K. Levin, "Pairing fluctuation theory of superconducting properties in underdoped to overdoped cuprates," Phys. Rev. Lett., 81, 4708-4711 (1998).

105. A. S. Alexandrov and N. F. Mott, "Thermal transport in a charged Bose gas and in high-T ${ }_{c}$ oxides," Phys. Rev. Lett., 71, 1075-1078 (1993).

106. A. S. Alexandrov and N. F. Mott, "Bipolarons," Rep. Prog. Phys., 57, 1197$1288(1994)$

107. Y. J. Uemura, G. M. Luke, B. J. Sternlieb, J. H. Brewer, J. F. Carolan, W. N. Hardy, R. Kadono, J. R. Kempton, R. F. Kiefl, S. R. Kreitzman, P. Mulhern, T. M. Riseman, D. L. Williams, B. X. Yang, S. Uchida, H. Takagi, J. Gopalakrishnan, A. W. Sleight, M. A. Subramanian, C. L. Chien, M. Z. Cieplak, G. Xiao, V. Y. Lee, B. W. Statt, C. E. Stronach, W. J. Kossler, and $\mathrm{X}$. H. Yu, "Universal correlations between Tc and $\mathrm{ns} / \mathrm{m}^{*}$ (carrier density over effective mass) in high- $\mathrm{T}_{c}$ cuprate superconductors," Phys. Rev. Lett., 62, 2317-2320 (1989).

108. E. Dagotto, T. Hotta, and A. Moreo, "Colossal magnetoresistant materials: the key role of phase separation," Physics Reports, 344, 1-153 (2001).

109. J. M. Luttinger, "Fermi surface and some simple equilibrium properties of a system of interacting fermions," Phys. Rev., 119, 1153-1163 (1960). 
110. V. J. Emery and S. A. Kivelson, "Crossovers and phase coherence in cuprate superconductors," J. Phys. Chem. Solids, 59, 1705-1710 (1998).

111. A. N. Kocharian, C. Yang, and Y. L. Chiang, "Self-consistent and exact studies of pairing correlations and crossover in the one-dimensional attractive Hubbard model," Phys. Rev. B, 59, 7458-7472 (1999).

112. A. N. Kocharian, C. Yang, and Y. L. Chiang, "Phase diagram and BCS-Bose condensation crossover in $1 \mathrm{D}$ and 2D Hubbard models," Physica $C, \mathbf{3 6 4 - 3 6 5}$, 131-133 (2001).

113. J. W. Allen, C. G. Olson, M. B. Maple, J.-S. Kang, L. Z. Liu, J.-H. Park, R. O. Anderson, W. P. Ellis, J. T. Market, Y. Dalichaouch, and R. Liu, "Resonant photoemission study of $\mathrm{Nd}_{2-x} \mathrm{Ce}_{x} \mathrm{CuO}_{4-y}$ : Nature of electronic states near the Fermi level," Phys. Rev. Lett., 64, 595-598 (1990).

114. R. O. Anderson, R. Cleassen, J. W. Allen, C. G. Olson, C. Janowitz, L. Z. Liu, J.-H. Park, M. B. Maple, Y. Dalichaouch, M. C. de Andrade, R. F. Jardim, E. A. Early, S.-J. Ho, and W. P. Ellis, "Luttinger Fermi surface of metallic gap spectral weight in $\mathrm{Nd}_{1.85} \mathrm{Ce}_{0.15} \mathrm{CuO}_{4-y}$," Phys. Rev. Lett., 70, 3163-3166 (1993).

115. T. Watanabe, T. Takahashi, S. Suzuki, S. Sato, and H. Katayama-Yoshida, "Inverse-photoemission study of hole-concentration dependence of the electronic structure in $\mathrm{Bi}_{2} \mathrm{Sr}_{2} \mathrm{Ca}_{1-x} \mathrm{Y}_{x} \mathrm{Cu}_{2} \mathrm{O}_{8}(\mathrm{x}=0.0-0.05), "$ Phys. Rev. B, 44, 5316-5317 (1991).

116. A. Ino, C. Kim, M. Nakamura, T. Yoshida, T. Mizokawa, Z.-X. Shen, A. Fujimori, T. Kakeshita, H. Eisaki, and S. Uchida, "Electronic structure of $\mathrm{La}_{2-x} \mathrm{Sr}_{x} \mathrm{CuO}_{4}$ in the vicinity of the superconductor-insulator transition," Phys. Rev. B, 62, 4137-4141 (2000).

117. G. Rietveld, N. Y. Chen, and D. van der Marel, "Anomalous temperature dependence of the work function in $\mathrm{YBa}_{2} \mathrm{Cu}_{3} \mathrm{O}_{7-\delta}$," Phys. Rev. B, 69, 25782581 (1992), a rather larger value of the chemical potential shift at $T_{c}$, but still very small compared to the bandwidth, was obtained from high precision measurements of the work function.

118. D. J. Scalapino, J. E. Loh, and J. E. Hirsch, "d-wave pairing near a spin density wave instability," Phys. Rev. B, 34, 8190-8192 (1986).

119. W. Kohn and J. M. Luttinger, "New mechanism for superconductivity," Phys. Rev. Lett., 15, 524-526 (1965).

120. V. J. Emery, "Theory of high-T $\mathrm{T}_{c}$ superconductivity in oxides," Phys. Rev. Lett., 58, 2794-2797 (1987).

121. G. Kotliar and J. Liu, "Superexchange mechanism and d-wave superconductivity," Phys. Rev. B, 38, 5142-5145 (1988).

122. C. Gros, R. Joynt, and T. M. Rice, "Superconductivity instability in the largeU limit of the two-dimensional Hubbard model," Z. Phys. B, 68, 425-432 (1987).

123. D. J. Scalapino, E. Loh, and J. E. Hirsch, "Fermi-surface instabilities and superconducting d-wave pairing," Phys. Rev. B, 35, 6694-6698 (1987).

124. M. Grilli, R. Raimondi, C. Castellani, C. Di Castro, and G. Kotliar, "Superconductivity, phase separation, and charge-transfer instability in the $\mathrm{U}=\infty$ limit of the three-band model of the $\mathrm{CuO}_{2}$ planes," Phys. Rev. Lett., 67, 259-262 (1991).

125. A. Perali, C. Castellani, C. Di Castro, and M. Grilli, " $d$-wave superconductivity near charge instabilities," Phys. Rev. B, 54, 16216-16225 (1996). 
126. D. A. Wollman, D. J. V. Harlingen, W. C. Lee, D. M. Ginsberg, and A. J. Leggett, "Experimental determination of the superconducting pairing state in YBCO from phase coherence in YBCO-Pb SQUIDs," Phys. Rev. Lett., 71, 2134-2147 (1993).

127. C. C. Tsuei, J. R. Kirtley, C. C. Chi, L. S. Yu-Jahnes, A. Gupta, T. Shaw, J. Z. Sun, and M. B. Ketchen, "Pairing symmetry and flux quantization in a tricrystal superconducting ring of $\mathrm{YBa}_{2} \mathrm{Cu}_{3} \mathrm{O}_{7-\delta}$," Phys. Rev. Lett., 73, 593 (1994).

128. K. A. Kouznetsov, A. G. Sun, B. Chen, A. S. Katz, S. R. Bahcall, J. Clarke, R. C. Dynes, D. A. Gajewski, S. H. Han, M. B. Maple, J. Giapintzakis, J.-T. Kim, and D. M. Ginsberg, "C-axis Josephson tunneling between $\mathrm{YBa}_{2} \mathrm{Cu}_{3} \mathrm{O}_{7-\delta}$ and $\mathrm{Pb}$ : direct evidence for mixed order parameter symmetry in a high- $T_{c}$ superconductor," Phys. Rev. Lett., 79, 3050-3053 (1997).

129. R. A. Klemm, G. Arnold, A. Bille, and K. Scharnberg, "Theory of c-axis twist Bi2212 Josephson junctions: Strong evidence for incoherent tunneling and s-wave superconductivity," Physica C, 341, 1663-1664 (2000).

130. A. Damascelli, D. H. Lu, and Z.-X. Shen, "From Mott insulator to overdoped superconductor: Evolution of the electronic structure of cuprates studied by ARPES," J. Electron Spectr. Relat. Phenom., 165, 117-118 (2001).

131. J. R. Schrieffer, S. C. Zhang, and X. G. Wen, "Spin-bag mechanism of hightemperature superconductivity," Phys. Rev. Lett., 60, 944-947 (1988).

132. M. Granath, V. Oganesyan, S. A. Kivelson, E. Fradkin, and V. J. Emery, "Nodal quasiparticles in stripe ordered superconductors," Phys. Rev. Lett., 87, 167011-167014 (2001).

133. M. Vojta, Y. Zhang, and S. Sachdev, "Quantum phase transitions in d-wave superconductors," Phys. Rev. Lett., 85, 4940-4943 (2000).

134. M. Kugler, O. Fischer, C. Renner, S. Ono, and Y. Ando, "Scanning tunneling spectroscopy of $\mathrm{Bi}_{2} \mathrm{Sr}_{2} \mathrm{CuO} 6+\delta$ : new evidence for the common origin of the pseudogap and superconductivity," Phys. Rev. Lett., 86, 4911 (2001).

135. N. J. Curro, P. C. Hammel, B. J. Suh, M. Hucker, B. Buchner, U. Ammerahl, and A. Revcolevschi, "Inhomogeneous low frequency spin dynamics in $\mathrm{La}_{1.65} \mathrm{Eu}_{0.2} \mathrm{Sr}_{0.15} \mathrm{CuO}_{4}, "$ Phys. Rev. Lett., 85, 642-645 (2000).

136. H. Takagi, B. Batlogg, H. L. Kao, J. Kwo, R. J. Cava, J. J. Krajewski, and W. F. P. Jr., "Systematic evolution of temperature-dependent resistivity in $\mathrm{La}_{2-x} \mathrm{Sr}_{x} \mathrm{CuO}_{4}, "$ Phys. Rev. Lett., 69, 2975-2978 (1992).

137. B. Bucher, P. Steiner, J. Karpinski, E. Kaldis, and P. Wachter, "Influence of the spin gap on the normal state transport in $\mathrm{YBa}_{2} \mathrm{Cu}_{4} \mathrm{O}_{8}$," Phys. Rev. Lett., 70, 2012-2015 (1993).

138. K. Takenaka, K. Mizuhashi, H. Takagi, and S. Uchida, "Interplane charge transport in $\mathrm{YBa}_{2} \mathrm{Cu}_{3} \mathrm{O}_{7-y}$ : Spin-gap effect on in-plane and out-of-plane resistivity," Phys. Rev. B, 50, 6534-6537 (1994).

139. A. N. Lavrov, Y. Ando, and S. Ono, "Two mechanisms of pseudogap formation in Bi-2201: evidence from the c-axis magnetoresistance," Euro. Phys. Lett, 57, 267-273 (2002).

140. J. W. Loram, K. A. Mirza, J. R. Cooper, and W. Y. Liang, "Electronic specific heat of $\mathrm{YBa}_{2} \mathrm{Cu}_{3} \mathrm{O}_{6+x}$ from 1.8 to $300 \mathrm{~K}$," Phys. Rev. Lett., 71, 1740-1743 (1993).

141. J. Orenstein, G. A. Thomas, A. J. Millis, S. L. Cooper, D. H. Rapkine, T. Timusk, L. F. Schneemeyer, and J. V. Waszczak, "Frequency- and 
temperature-dependent conductivity in $\mathrm{YBa}_{2} \mathrm{Cu}_{3} \mathrm{O}_{6+x}$ crystals," Phys. Rev. $B, \mathbf{4 2}, 6342-6362$ (1990).

142. A. Puchkov, D. N. Basov, and T. Timusk, "Pseudogap state in high-T superconductors: an infrared study," J.Phys Cond. Matt., 8, 10049 (1996).

143. C. C. Homes, T. Timusk, R. Liang, D. A. Bonn, and W. N. Hardy, "Optical conductivity of c axis oriented $\mathrm{YBa}_{2} \mathrm{Cu}_{3} \mathrm{O}_{6.70}$ : Evidence for a pseudogap," Phys. Rev. Lett., 71, 1645-1648 (1993).

144. D. Basov, H. A. Mook, B. Dabrowski, and T. Timusk, "c-axis response of single- and double-layered cuprates," Phys. Rev. B, 52, R13141-R13144 (1995).

145. M. Arai, T. Nishijima, Y. Endoh, T. Egami, S. Tajima, K. Tomimoto, Y. Shiohara, M. Takahashi, A. Garrett, and S. M. Bennington, "Incommensurate spin dynamics of underdoped superconductor $\mathrm{YBa}_{2} \mathrm{Cu}_{3} \mathrm{O}_{6.7}$," Phys. Rev. Lett., 83, 608-611 (1999).

146. P. C. Dai, H. A. Mook, S. M. Hayden, G. Aeppli, T. G. Perring, R. D. Hunt, and F. Doğan, "The magnetic excitation spectrum and thermodynamics of high $T_{c}$ superconductors," Science, 284, 1344-1347 (1999).

147. B. Batlogg and V. J. Emery, "Crossovers in cuprates," Nature, 382, 20 (1996).

148. X. G. Wen and P. A. Lee, "Theory of underdoped cuprates," Phys. Rev. Lett., 76, 503-506 (1996).

149. E. W. Carlson, D. Orgad, S. A. Kivelson, and V. J. Emery, "Dimensional crossover in quasi-one-dimensional and high $T_{c}$ superconductors," Phys. Rev. $B, 62,3422-3437$ (2000).

150. B. G. Levi, Physics Today, 49, 17 (1996).

151. D.-H. Lee, "Superconductivity in a doped Mott insulator," Phys. Rev. Lett., 84, 2694-2697 (2000).

152. S. Sachdev, "Kagome-acute- and triangular-lattice Heisenberg antiferromagnets: Ordering from quantum fluctuations and quantum-disordered ground states with unconfined bosonic spinons," Phys. Rev. B, 45, 12377-12396 (1992).

153. M. U. Ubbens and P. A. Lee, "Superconductivity phase diagram in the gaugefield description of the t-J model," Phys. Rev. B, 49, 6853-6863 (1994).

154. S.-C. Zhang, "SO(5) quantum nonlinear sigma model theory of the high $\mathrm{T}_{c}$ superconductivity," Science, 275, 1089-1096 (1997).

155. S.-C. Zhang, J.-P. Hu, E. Arrigoni, W. Hanke, and A. Auerbach, "Projected SO(5) models," Phys. Rev. B, 60, 13070-13084 (1999).

156. A. Auerbach and E. Altman, "Projected SO(5) Hamiltonian for cuprates and its applications," Int. Jour. Mod. Phys. B, 15, 2509-2518 (2001).

157. J. Ye, "Thermally generated vortices, gauge invariance, and electron spectral function in the pseudogap regime," Phys. Rev. Lett., 87, 227003-227006 (2001).

158. A. Abanaov, A. V. Chubukov, and J. Schmalian, "Fingerprints of spin mediated pairing in cuprates," Journal of Electron Spectroscopy and Related Phenomena, 117-118, 129-151 (2001).

159. M. Takigawa, A. P. Reyes, P. C. Hammel, J. D. Thompson, R. H. Heffner, Z. Fisk, and K. C. Ott, "Cu and O NMR studies of the magnetic properties of $\mathrm{YBa}_{2} \mathrm{Cu}_{3} \mathrm{O}_{6.63}\left(T_{c}=62 \mathrm{~K}\right)$," Phys. Rev. B, 43, 247-257 (1991).

160. H. A. Mook and F. Dogan, "Charge fluctuations in $\mathrm{YBa}_{2} \mathrm{Cu} 3 \mathrm{O}_{7-x}$ hightemperature superconductors," Nature, 401, 145-148 (1999). 
161. P. Dai, H. A. Mook, and F. Dogan, "Incommensurate magnetic fluctuations in $\mathrm{YBa}_{2} \mathrm{Cu}_{3} \mathrm{O}_{6.6}$," Phys. Rev. B, 80, 1738-1741 (1998).

162. J. M. Tranquada, P. M. Gehring, G. Shirane, S. Shamoto, and M. Sato, "Neutron-scattering study of the dynamical spin susceptibility in $\mathrm{YBa}_{2} \mathrm{Cu}_{3} \mathrm{O}_{6.6}, "$ Phys. Rev. B, 46, 5561-5575 (1992).

163. H. A. Mook, P. Dai, and F. Dogan, "Charge and spin structure in $\mathrm{YBa}_{2} \mathrm{Cu}_{3} \mathrm{O}_{6.35}$," Phys. Rev. Lett., 88, 097004-097007 (2002).

164. S. Wakimoto, R. J. Birgeneau, M. A. Kastner, Y. S. Lee, R. Erwin, P. M. Gehring, S. H. Lee, M. Fujita, K. Yamada, Y. Endoh, K. Hirota, and G. Shirane, "Direct observation of a one-dimensional static spin modulation in insulating $\mathrm{La}_{1.95} \mathrm{Sr}_{0.05} \mathrm{CuO}_{4}$," Phys. Rev. B, 61, 3699-3706 (2000).

165. H. Kimura, H. Matsushita, K. Hirota, Y. Endoh, K. Yamada, G. Shirane, Y. S. Lee, M. A. Kastner, and R. J. Birgeneau, "Incommensurate geometry of the elastic magnetic peaks in superconducting $\mathrm{La}_{1.8} \mathrm{Sr}_{0.12} \mathrm{CuO}_{4}, "$ Phys. Rev. B, 61, 14366-14369 (2000).

166. B. O. Wells, Y. S. Lee, M. A. Kastner, R. J. Christianson, R. J. Birgeneau, K. Yamada, Y. Endoh, and G. Shirane, "Incommensurate spin fluctuations in high-transition temperature superconductors," Science, 277, 1067-1071 (1997).

167. M. Matsuda, M. Fujita, K. Yamada, R. J. Birgeneau, Y. Endoh, and G. Shirane, "Electronic phase separation in lightly doped $\mathrm{La}_{2-x} \mathrm{Sr}_{x} \mathrm{CuO}_{4}$," Phys. Rev. B, 65, 134515 (2002).

168. P. A. Lee and N. Nagaosa, "Gauge theory of the normal state of high- $\mathrm{T}_{c}$ superconductors," Phys. Rev. B, 46, 5621-39 (1992).

169. C. Meingast, V. Pasler, P. Nagel, A. Rykov, S. Tajima, and P. Olsson, "Phase fluctuations and the pseudogap in $\mathrm{YBa}_{2} \mathrm{Cu}_{3} \mathrm{O}_{7-\delta}$," Phys. Rev. Lett., 86, 16061609 (2001).

170. J. Corson, R. Mallozzi, J. Orenstein, J. N. Eckstein, and I. Bozovic, "Vanishing of phase coherence in underdoped $\mathrm{Bi}_{2} \mathrm{Sr}_{2} \mathrm{CaCu}_{2} \mathrm{O}_{8+\delta}$," Nature, 398, 221-223 (1999).

171. D. A. Bonn, S. Kamal, A. Bonakdarpour, L. Ruixing, W. N. Hardy, C. C. Homes, and D. N. B. T. Timusk, "Surface impedance studies of YBCO," Czech. J. Phys., 46, 3195-3202 (1996).

172. V. Pasler, P. Schweiss, C. Meingast, B. Obst, H. Wuhl, A. I. Rykov, and S. Tajima, "3D-XY critical fluctuations of the thermal expansivity in detwinned $\mathrm{YBa}_{2} \mathrm{Cu}_{3} \mathrm{O}_{7-\delta}$ single crystals near optimal doping," Phys. Rev. Lett., 81, 1094-1097 (1998).

173. C. Varma, "Pseudogap phase and the quantum-critical point in copper-oxide metals," Phys. Rev. Lett., 83, 3538-3541 (1999).

174. S. Chakravarty, unpublished.

175. T. Valla, P. D. Johnson, Z. Yusof, B. Wells, Q. Li, S. M. Loureiro, R. J. Cava, M. Mikami, Y. Mori, M. Yoshimura, and T. Sasaki, "Coherence-incoherence and dimensional crossover in layered strongly correlated metals," submitted to Science (2002).

176. J. Zaanen, "Superconductivity: self-organized one dimensionality," Science, 286, 251-252 (1999).

177. Preliminary evidence of the existence of nematic order in $\mathrm{La}_{2-x} \mathrm{Sr}_{x} \mathrm{CuO}_{4}$ and $\mathrm{YBa}_{2} \mathrm{Cu}_{3} \mathrm{O}_{7-\delta}$ can be found in 998 . 
178. J. P. Eisenstein, M. P. Lilly, K. B. Cooper, L. N. Pfeiffer, and K. W. West, "New collective states of 2D electrons in high Landau levels," Physica E, 9, 1-8 (2001).

179. M. M. Fogler, "Stripe and bubble phases in quantum Hall systems," condmat/0111001 (2001).

180. M. M. Fogler, "Quantum Hall liquid crystals," cond-mat/0111049 (2001).

181. V. J. Emery, "Theory of the one-dimensional electron gas," in "Highly Conducting One-Dimensional Solids," edited by J. T. Devreese, R. P. Evrard, and V. E. van Doren, 327 (Plenum, New York) (1979).

182. J. Solyom, "The Fermi gas model of one-dimensional conductors," Adv. Phys., 28, 201-303 (1979).

183. E. Fradkin, Field Theories of Condensed Matter Systems (Addison-Wesley, Massachusetts) (1991).

184. J. von Delft and H. Schoeller, "Bosonization for beginners - Refermionization for experts," Annalen Phys., 7, 225-305 (1998).

185. A. O. Gogolin, A. A. Nersesyan, and A. M. Tsvelik, Bosonization and Strongly Correlated Systems (Cambridge University Press, Cambridge) (1998).

186. H. J. Schulz, G. Guniberti, and P. Pieri, "Fermi liquids and Luttinger liquids," cond-mat/9807366 (1998).

187. J. Voit, "One-dimensional Fermi liquids," Rep. Prog. Phys., 58, 977-1116 (1995).

188. J. M. Kosterlitz and D. J. Thouless, "Ordering, metastability and phase transitions in two-dimensional systems," J. Phys. C, 6, 1181-1203 (1973).

189. T. Giamarchi and H. J. Schulz, "Correlation functions of one-dimensional quantum systems," Phys. Rev. B, 39, 4620-4629 (1989).

190. A. Luther and V. J. Emery, "Backward scattering in the one-dimensional electron gas," Phys. Rev. Lett., 33, 589-592 (1974).

191. N. Kawakami and S. K. Yang, "Luttinger anomaly exponent of momentum distribution in the Hubbard chain," Phys. Lett. A, 148, 359-362 (1990).

192. R. M. Noack, N. Bulut, D. J. Scalapino, and M. G. Zacher, "Enhanced $d_{x^{2}-y^{2}}$ pairing correlations in the two-leg Hubbard ladder," Phys. Rev. B, 56, 71627166 (1997).

193. T. Valla, A. V. Fedorov, P. D. Johnson, and S. L. Hulber, "Many-body effects in angle-resolved photoemission: Quasiparticle energy and lifetime of a Mo(110) surface state," Phys. Rev. Lett., 83, 2085-2088 (1999).

194. A. Luther and I. Peschel, "Single-particle states, Kohn anomaly and pairing fluctuations in one dimension," Phys. Rev. B, 9, 2911-2919 (1974).

195. V. Meden and K. Schönhammer, "Spectral functions for the TomonagaLuttinger model," Phys. Rev. B, 46, 15753-15760 (1992).

196. J. Voit, "Charge-spin separation and the spectral properties of Luttinger liquids," Phys. Rev. B, 47, 6740-6743 (1993).

197. D. Orgad, "Spectral functions for the Tomonga-Luttinger and Luther-Emery liquids," Phil. Mag. B, 81, 377-398 (2001).

198. G.-H. Gweon, J. W. Allen, and J. D. Denlinger, "Ubiquitous generalized spectral signatures of electron fractionalization in quasi-low dimensional metals,"

199. F. H. L. Essler and A. M. Tsvelik, "Weakly coupled one-dimensional Mott insulators," Phys. Rev. B, 65, 115117-115129 (2002).

200. D. J. Scalapino, Y. Imry, and P. Pincus, "Generalized Ginzburg-Landau theory of pseudo-one-dimensional systems," Phys. Rev. B, 11, 2042-2048 (1975). 
201. E. Arrigoni, "Crossover to Fermi-liquid behavior for weakly coupled Luttinger liquids in the anisotropic large-dimension limit," Phys. Rev. B, 61, 7909-7029 (2000).

202. D. L. Feng, D. H. Lu, K. M. Shen, C. Kim, H. Eisaki, A. Damascelli, R. Yoshizaki, J. Shimoyama, K. Kishio, G. D. Gu, S. Oh, A. Andrus, J. O'Donnell, J. N. Eckstein, and Z. X. Shen, "Signature of superfluid density in the single-particle excitation spectrum of $\mathrm{Bi}_{2} \mathrm{Sr}_{2} \mathrm{CaCu}_{2} \mathrm{O}_{8+\delta}$," Science, 289, 277-281 (2000).

203. H. Ding, J. R. Engelbrecht, Z. Wang, J. C. Campuzano, S.-C. Wang, H.-B. Yang, R. Rogan, T. Takahashi, K. Kadowaki, and D. G. Hinks, "Coherent quasiparticle weight and its connection to high- Tc superconductivity from angle-resolved photoemission," Phys. Rev. Lett., 87, 227001-227004 (2000).

204. S. Biermann, A. Georges, A. Lichtenstein, and T. Giamarchi, "Deconfinement transition and Luttinger to Fermi liquid crossover in quasi-one-dimensional systems," Phys. Rev. Lett., 87, 276405-276408 (2001).

205. S. Biermann, A. Georges, T. Giamarchi, and A. Lichtenstein, "Quasi-onedimensional organic conductors: dimensional crossover and some puzzles," cond-mat/0201542 (2002).

206. L. Yin and S. Chakravarty, "Spectral anomaly and high temperature superconductors," Int. J. Mod. Phys. B, 7, 805-45 (1996).

207. V. J. Emery, E. Fradkin, S. A. Kivelson, and T. C. Lubensky, "Quantum theory of the smectic metal state in stripe phases," Phys. Rev. Lett., 85, 2160-2163 (2000).

208. A. H. Castro Neto, "Stripes, vibrations, and superconductivity," Phys. Rev. $B, 64,104509-104535$ (2001).

209. A. Vishwanath and D. Carpentier, "Two-Dimensional anisotropic non-Fermiliquid phase of coupled Luttinger liquids," Phys. Rev. Lett., 86, 676-679 (2001).

210. C. S. O'Hern, J. Toner, and T. C. Lubensky, "Sliding phases in XY Models, crystals, and cationic lipid-DNA complexes," Phys. Rev. Lett., 83, 2745-2748 (1999).

211. R. Mukhopadhyay, C. L. Kane, and T. C. Lubensky, "Sliding Luttinger liquid phases," Phys. Rev. B, 64, 045120-045137 (2001).

212. S. L. Sondhi and K. Yang, "Sliding phases via magnetic fields," Phys. Rev. B, 63, 054430-054436 (2001).

213. S. A. Kivelson, "Electron fractionalization," Synth. Met., 125 (2001).

214. T. Senthil and O. Motrunich, "Microscopic models for fractionalized phases in strongly correlated systems," cond-mat/0201320 (2002).

215. P. Fazekas and P. W. Anderson, "On the ground state properties of the anisotropic triangular antiferromagnet. (Application of anisotropic Heisenberg model)," Phil. Mag., 30, 423-440 (1974).

216. R. B. Laughlin, "The relationship between high-temperature superconductivity and the fractional quantum Hall effect," Science, 242, 525-533 (1988).

217. N. Read and B. Chakraborty, "Statistics of the excitations of the resonatingvalence-bond state," Phys. Rev. B, 40, 7133-7140 (1989).

218. V. Kalmeyer and R. B. Laughlin, "Equivalence of the resonating-valence-bond and fractional quantum Hall states," Phys. Rev. Lett., 59, 2095-2098 (1987).

219. S. A. Kivelson, "Statistics of holons in the quantum hard-core dimer gas," Phys. Rev. B, 39, 259-264 (1989). 
220. E. Demler, C. Nayak, H.-Y. Kee, Y. B. Kim, and T. Senthil, "Fractionalization patterns in strongly correlated electron systems: Spin-charge separation and beyond," cond-mat/0105446 (2001).

221. F. D. M. Haldane, "O (3) nonlinear sigma model and the topological distinction between integer- and half-integer-spin antiferromagnets in two dimensions," Phys. Rev. Lett., 61, 1029-32 (1988).

222. S. Chakravarty, B. I. Halperin, and D. Nelson, "Two-dimensional quantum Heisenberg antiferromagnet at low temperatures," Phys. Rev. B, 39, 23442371 (1989).

223. S. Chakravarty, B. I. Halperin, and D. Nelson, "Low-temperature behavior of two-dimensional quantum antiferromagnets," Phys. Rev. Lett., 60, 1057-1060 (1988).

224. R. Coldea, S. M. Hayden, G. Aeppli, T. G. Perring, C. D. Frost, T. E. Mason, S.-W. Cheong, and Z. Fisk, "Spin waves and electronic interactions in $\mathrm{La}_{2} \mathrm{CuO}_{4}$," Phys. Rev. Lett., 86 (2001).

225. R. J. Birgeneau, A. Aharony, N. R. Belk, F. C. Chou, Y. Endoh, M. Greven, S. Hosoya, M. A. Kastner, C. H. Lee, Y. S. Lee, G. Shirane, S. Wakimoto, B. O. Wells, and K. Yamada, "Magnetism and magnetic fluctuations in $\mathrm{La}_{2-x} \mathrm{Sr}_{x} \mathrm{CuO}_{4}$ for $\mathrm{x}=0$ (2D antiferromagnet), 0.04 (3D spin glass) and $\mathrm{x}=0.15$ (superconductor)," Jour. Phys. Chem. Solids, 56, 1912-1919 (1995).

226. A. Weidinger, C. Niedermayer, A. Golnik, R. Simon, E. Recknagel, J. I. Budnick, B. Chamberland, and C. Baines, "Observation of magnetic ordering in superconducting $\mathrm{La}_{2-x} \mathrm{Sr}_{x} \mathrm{CuO}_{4}$ by muon spin rotation," Phys. Rev. Lett., 62 (1989).

227. C. Niedermayer, C. Bernhard, T. Blasius, A. Golnik, A. Moodenbaugh, and J. I. Budnick, "Common phase diagram for antiferromagnetism in $\mathrm{La}_{2-x} \mathrm{Sr}_{x} \mathrm{CuO}_{4}$ and $\mathrm{Y}_{1-x} \mathrm{Ca}_{x} \mathrm{Ba}_{2} \mathrm{Cu}_{3} \mathrm{O}_{6}$ as seen by muon spin rotation," Phys. Rev. Lett., 80 (1999).

228. C. Panagopoulos, J. L. Tallon, B. D. Rainford, T. Xiang, J. R. Cooper, and C. A. Scott, "Evidence for a generic novel quantum transition in high-Tc cuprates," cond-mat/0204106 (2002).

229. P. Azaria, C. Hooley, P. Lecheminant, C. Lhuillier, and A. M. Tsvelik, "Kagome lattice antiferromagnet stripped to its basics," Phys. Rev. Lett., 81, 1694-1697 (1998).

230. W. LiMing, G. Misguich, P. Sindzingre, and C. Lhuillier, "From Neel longrange order to spin liquids in the multiple-spin exchange model," Phys. Rev. $B, 62,6372-6377$ (2000).

231. G. Misguich, B. Bernu, C. Lhuillier, and C. Waldtmann, "Spin liquid in the multiple-spin exchange model on the triangular lattice: ${ }^{3} \mathrm{He}$ on graphite," Phys. Rev. Lett., 81, 1098-1101 (1998).

232. C. Lhuillier and G. Misguich, "Frustrated quantum magnets," condmat/0109146 (2001).

233. S. Chakravarty, S. A. Kivelson, C. Nayak, , and K. Volker, "Wigner glass, spin liquids and the metal-insulator transition," Phil. Mag. B, 79 (1999).

234. A. V. Chubukov, S. Sachdev, and T. Senthil, "Quantum phase transitions in frustrated quantumantiferromagnets," Nucl. Phys. B, 426, 601-643 (1994).

235. R. Meservey and B. B. Schwartz, "equilibrium properties: comparison of experimental results with predictions of the BCS theory," in "Superconductivity," edited by R. D. Parks, vol. 1 (Marcel Deckker, Inc., New York, NY) (1969). 
236. E. A. Lynton, Superconductivity (Methuen, London) (1962).

237. T. P. Orlando, E. J. McNiff Jr., S. Foner, and M. R. Beasley, "Critical fields, Pauli paramagnetic limiting, and material parameters of Nb3Sn and V3Si," Phys. Rev. B, 19, 4545-4561 (1979).

238. Y. G. Naidyuk and I. K. Yanson, "Point-contact spectroscopy of heavy-fermion systems," J. Phys. Cond. Matt., 10, 8905-8938 (1998).

239. M. B. Maple, J. W. Chen, S. E. Lambert, Z. Fisk, J. L. Smith, H. R. Ott, J. S. Brooks, and M. J. Naughton, "Upper critical magnetic field of the heavyfermion superconductor UBe13," Phys. Rev. Lett., 54, 477-480 (1985).

240. F. Gross, K. Andres, and S. Chandrasekhar, "Experimental determination of the absolute value of the London penetration depth in the heavy fermion superconductors $\mathrm{UBe}_{13}$ and $\mathrm{UPt}_{3}, "$ Physica $C$, 162-164, 419-420 (1989).

241. F. Sharifi, A. Pargellis, R. C. Dynes, B. Miller, E. S. Hellman, J. Rosamilia, and E. H. H. Jr., "Electron tunneling in the high-Tc bismuthate superconductors," Phys. Rev. B, 44, 12521-12524 (1991).

242. Y. J. Uemura, L. P. Le, G. M. Luke, B. J. Sternlieb, W. D. Wu, J. H. Brewer, T. M. Riseman, C. L. Seaman, M. B. Maple, M. Ishikawa, D. G. Hinks, J. D. Jorgensen, G. Saito, and H. Yamochi, "Basic similarities among cuprate, bismuthate, organic, chevrel-phase, and heavy-fermion superconductors shown by penetration-depth measurements," Phys. Rev. Lett., 66, 2665-2668 (1991).

243. O. Gunnarsson, "Superconductivity in fullerides," Rev. Mod. Phys., 69, 575606 (1997).

244. Y. J. Uemura, A. Keren, L. P. Le, G. M. Luke, B. J. Sternlieb, W. D. Wu, J. H. Brewer, R. L. Whetten, S. M. Huang, S. Lin, R. B. Kaner, F. Diederich, S. Donovan, G. Gruner, and K. Holczer, "Magnetic-field penetration depth in $\mathrm{K}_{3} \mathrm{C}_{60}$ measured by muon spin relaxation," Nature, 352, 605-607 (1991).

245. A. P. Ramirez, Superconductivity Rev., 1, 1-101 (1994).

246. A. S. Alexandrov, "Nonadiabatic polaronic superconductivity in $\mathrm{MgB}_{2}$ and cuprates," Physica C, 363, 231-236 (2001).

247. H. Schmidt, J. F. Zasadzinski, K. E. Gray, and D. G. Hinks, "Evidence for two-band superconductivity from break junction tunneling on $\mathrm{MgB}_{2}$," Phys. Rev. Lett., 88, 127002-127005 (2002).

248. A. V. Sologubenko, J. Jun, S. M. Kazakov, J. Karpinski, and H. R. Ott, "Temperature dependence and anisotropy of the bulk upper critical field $\mathrm{H}_{c 2}$ of $\mathrm{MgB}_{2}, "$ Phys. Rev. B, 65, R180505-R180508 (2002).

249. T. Arai, K. Ichimura, K. Nomur, S. Takasaki, J. Yamada, S. Nakatsuji, and H. Anzai, "Superconducting and normal-state gaps in $\kappa$-(BEDT$\mathrm{TTF})_{2} \mathrm{Cu}(\mathrm{NCS})_{2}$ studied by STM spectroscopy," Solid State Commun., 116, 679-682 (2000).

250. Y. J. Uemura, A. Keren, L. P. Le, G. M. Luke, W. D. Wu, Y. Kubo, T. Manako, Y. Shimakawa, M. Subramanian, J. L. Cobb, and J. T. Markert, "Magneticfield penetration depth in $\mathrm{Tl}_{2} \mathrm{Ba}_{2} \mathrm{CuO}_{6+\delta}$ in the overdoped regime," Nature, 364, 605-607 (1993).

251. R. Prozorov, R. W. Giannetta, A. Carrington, P. Fournier, R. L. Greene, P. Guptasarma, D. G. Hinks, and A. R. Banks, "Measurements of the absolute value of the penetration depth in high- $\mathrm{T}_{c}$ superconductors using a low- $\mathrm{T}_{c}$ superconductive coating," Appl. Phys. Lett., 77, 4202 (2000).

252. A. Biswas, P. Fournier, V. N. Smolyaninova, R. C. Budhani, J. S. Higgins, and R. L. Greene, "Gapped tunneling spectra in the normal state of $\mathrm{Pr}_{2-x} \mathrm{Ce}_{x} \mathrm{CuO}_{4}$," Phys. Rev. B, 64, 104519-104526 (2001). 
253. Kajitani, K. Hiraga, S. Hosoya, and T. F. K. O.-I. Y. Syono, "Structural study of oxygen-saturated or quenched $\mathrm{Pr}_{2-x} \mathrm{Ce}_{x} \mathrm{CuO}_{4}$ with $\mathrm{x}_{i}=0.15$," Physica $C$, 178, 397-404 (1991).

254. L. Ozyuzer, Z. Yusof, J. F. Zasadzinski, R. Mogilevsky, D. G. Hinks, and K. E. Gray, "Evidence of $d_{x^{2}-y^{2}}$ symmetry in the tunneling conductance density of states of $\mathrm{Tl}_{2} \mathrm{Ba}_{2} \mathrm{CuO}_{6}$," Phys. Rev. B, 57, R3245-R3248 (1998).

255. C. Niedermayer, C. Bernhard, U. Binninger, H. Glckler, J. L. Tallon, E. J. Ansaldo, and J. I. Budnick, "Muon spin rotation study of the correlation between $\mathrm{Tc}$ and $\mathrm{ns} / \mathrm{m}^{*}$ in overdoped $\mathrm{Tl}_{2} \mathrm{Ba}_{2} \mathrm{CuO}_{6+\delta}$," Phys. Rev. Lett., 71, 1764 (1993).

256. M. Kang, G. Blumberg, M. V. Klein, and N. N. Kolesnikov, "Resonance Raman study of the superconducting gap and low energy excitations in $\mathrm{Tl}_{2} \mathrm{Ba}_{2} \mathrm{CuO}_{6+\delta}$ superconductors," Phys. Rev. Lett., 77, 4434 (1996).

257. N. Miyakawa, P. Guptasarma, J. F. Zasadzinski, D. G. Hinks, and K. E. Gray, "Strong dependence of the superconducting gap on oxygen doping from tunneling measurements on $\mathrm{Bi}_{2} \mathrm{Sr}_{2} \mathrm{CaCu}_{2} \mathrm{O}_{8-\delta}$," Phys. Rev. Lett., 80, 157-160 (1998).

258. M. Niderost, R. Frassanito, M. Saalfrank, A. C. Mota, G. Blatter, V. N. Zavaritsky, T. W. Li, and P. H. Kes, "Lower critical field $\mathrm{H}_{c 1}$ and barriers for vortex entry in $\mathrm{Bi}_{2} \mathrm{Sr}_{2} \mathrm{CaCu}_{2} \mathrm{O}_{8+\delta}$ crystals," Phys. Rev. Lett., 81, 3231 (1998).

259. S. L. Lee, P. Zimmermann, H. Keller, M. Warden, I. M. Savic, R. Schauwecker, D. Zech, R. Cubitt, E. M. Forgan, P. H. Kes, T. W. Li, A. A. Menovsky, and Z. Tarnawski, "Evidence for flux-lattice melting and a dimensional crossover in single-crystal $\mathrm{Bi}_{2.15} \mathrm{Sr}_{1.85} \mathrm{CaCu}_{2} \mathrm{O}_{8+\delta}$ from muon spin rotation studies," Phys. Rev. Lett., 71, 3862-3865 (1993).

260. M. Weber, P. Birrer, F. N. Gygax, B. Hitti, E. Lippelt, H. Maletta, and A. Schenck, "Measurements of the London penetration depth in Bi-based high$\mathrm{T}_{c}$ compounds," Hyp. Int., 63, 93 (1993).

261. C. Bernhard, J. L. Tallon, T. Blasius, A. Golnik, and C. Neidermayer, "Anomalous peak in the superconducting condensate density of cuprate highTc superconductors at a unique doping state," Phys. Rev. Lett., 86, 1614-1617 (2001).

262. C. Panagopoulos, J. R. Cooper, and T. Xiang, "Systematic behavior of the inplane penetration depth in d-wave cuprates," Phys. Rev. B, 57, 13422-13425 (1998).

263. P. Zimmermann, H. Keller, S. I. Lee, I. M. Savic, M. Warden, D. Zech, R. Cubitt, E. M. Forgan, E. Kaldis, J. Karpinski, and C. Kruger, "Muon-spinrotation studies of the temperature dependence of the magnetic penetration depth in the $\mathrm{YBa}_{2} \mathrm{Cu}_{3} \mathrm{O}_{x}$ family and related compounds," Phys. Rev. B, 52, 541-552 (1995).

264. J. Y. T. Wei, C. C. Tsuei, P. J. M. van Bentum, Z. Xiong, C. W. Chu, and M. K. Wu, "Quasiparticle tunneling spectra of the high-Tc mercury cuprates: Implications of the d-wave two-dimensional van Hove scenario," Phys. Rev. $B, \mathbf{5 7}, 3650-3662(1998)$.

265. L. Fàbrega, A. Calleja, A. Sin, S. P. nol, X. Obradors, J. Fontcuberta, and P. J. C. King, "Muon spin relaxation in Re-substituted $\mathrm{HgA}_{2} \mathrm{Ca}_{n-1} \mathrm{Cu}_{n} \mathrm{O}_{2 n+2+x}(\mathrm{~A}=\mathrm{Sr}, \mathrm{Ba} ; \mathrm{n}=2,3)$ superconductors," Phys. Rev. $B, 60,7579-7584$ (1999). 
266. A. Fujimori, A. Ino, T. Yoshida, T. Mizokawa, M. Nakamura, C. Kim, Z. X. Shen, T. Kakeshita, H. Eisaki, and S. Uchida, "Fermi surface, pseudogap and superconducting gap in $\mathrm{La}_{2-x} \mathrm{Sr}_{x} \mathrm{CuO}_{4}, "$ Physica C, 341-348, 2067 (2000).

267. C. Panagopoulos, B. D. Rainford, J. R. Cooper, W. Lo, J. L. Tallon, J. W. Loram, J. Betouras, Y. S. Wang, and C. W. Chu, "Effects of carrier concentration on the superfluid density of high-Tc cuprates," Phys. Rev. B, 60, 14617-14620 (1999).

268. P. G. Radaelli, D. G. Hinks, A. W. Mitchell, B. A. Hunter, J. L. Wagner, B. Dabrowski, K. G. Vandervoort, H. K. Viswanathan, and J. D. Jorgensen, "Structural and superconducting properties of $\mathrm{La}_{2-x} \mathrm{Sr}_{x} \mathrm{CuO}_{4}$ as a function of Sr content," Phys. Rev. B, 49, 4163-4175 (1994).

269. V. J. Emery and S. A. Kivelson, "Importance of phase fluctuations in superconductors with small superfluid density," Nature, 374, 434-437 (1995).

270. L. M. Merchant, J. Ostrick, R. P. B. Jr., and R. C. Dynes, "Crossover from phase fluctuation to amplitude-dominated superconductivity: A model system," Phys. Rev. B, 63, 134508-134514 (2001).

271. A. J. Rimberg, T. R. Ho, C. Kurdak, J. Clarke, K. L. Campman, and A. C. Gossard, "Dissipation-driven superconductor-insulator transition in a two-dimensional Josephson-junction array," Phys. Rev. Lett., 78, 2632-2635 (1997).

272. A. Kapitulnik, N. Mason, S. A. Kivelson, and S. Chakravarty, "Effects of dissipation on quantum phase transitions," Phys. Rev. B, 63, 125322-125333 (2001).

273. N. Mason and A. Kapitulnik, "True superconductivity in a two-dimensional superconducting-insulating system," Phys. Rev. B, 64, 60504-60508 (2001).

274. E. W. Carlson, S. A. Kivelson, V. J. Emery, and E. Manousakis, "Classical phase fluctuations in high temperature superconductors," Phys. Rev. Lett., 83, 612-615 (2000).

275. B. I. Spivak and S. A. Kivelson, "Aharonov-Bohm oscillations with period hc/4e and negative magnetoresistance in dirty superconductors," Phys. Rev. $B$, 45, 10490-10495 (1992), electron correlations could change this assumption.

276. P. M. Chaikin and T. C. Lubensky, Principles of condensed matter physics (Cambridge) (1995).

277. E. Roddick and D. Stroud, "Effect of phase fluctuations on the lowtemperature penetration depth of high- Tc superconductors," Phys. Rev. Lett., 74, 1430-1433 (1995).

278. M. W. Coffey, "Effect of superconductor phase fluctuations upon penetration depth," Phys. Lett. A, 200, 195-200 (1995).

279. S. Kamal, D. A. Bonn, N. Goldenfeld, P. J. Hirschfeld, R. Liang, and W. N. Hardy, "Penetration depth measurements of 3D XY critical behavior in $\mathrm{YBa}_{2} \mathrm{Cu}_{3} \mathrm{O}_{6.95}$ crystals," Phys. Rev. Lett., 73, 1845-1848 (1994).

280. S. Chakravarty, G. Ingold, S. A. Kivelson, and A. Luther, "Onset of global phase coherence in Josephson-junction arrays: a dissipative phase transition," Phys. Rev. Lett., 56 (1986).

281. K.-H. Wagenblast, A. V. Otterlo, G. Schon, and G. T. Zimanyi, "Superconductor-insulator transition in a tunable dissipative environment," Phys. Rev. Lett., 79 (1997).

282. S. Sachdev, Quantum Phase Transitions (Cambridge University Press, Cambridge, UK) (1999). 
283. M. V. Feigel'man, A. I. Larkin, and A. I. and M. A. Skvortsov, "Quantum superconductor-metal transition in a proximity array," Phys. Rev. Lett., 86, 1869-1872 (2001).

284. B. I. Spivak, A. Zyuzin, and M. Hruska, "Quantum superconductor-metal transition," Phys. Rev. B, 64, 132502-132505 (2001).

285. Y. Oreg and E. Demler, "Fermions and bosons in superconducting amorphous wires," cond-mat/0106645 (2001).

286. L. Ozyuzer, J. F. Zasadzinski, and N. Miyakawa, "Tunneling spectra and superconducting gap in $\mathrm{Bi}_{2} \mathrm{Sr}_{2} \mathrm{CaCu}_{2} \mathrm{O}_{8+\delta}$ and $\mathrm{Tl}_{2} \mathrm{Ba}_{2} \mathrm{CuO}_{6+\delta}$," Int. J. Mod. Phys. B, 13, 3721-3724 (1999).

287. S. Doniach and M. Inui, "Long-range Coulomb interactions and the onset of superconductivity in the high-Tc materials," Phys. Rev. B, 41, 6668-6678 (1990).

288. J. M. Harris, P. J. White, Z.-X. Shen, H. Ikeda, R. Yoshizaki, H. Eisaki, S. Uchida, W. D. Si, J. W. Xiong, Z.-X. Zhao, and D. S. Dessau, "Measurement of an anisotropic energy gap in single plane $\mathrm{Bi}_{2} \mathrm{Sr}_{2-x} \mathrm{La}_{x} \mathrm{CuO}_{6+\delta}$," Phys. Rev. Lett., 79, 143-146 (1997).

289. T. Schneider and H. Keller, "Extreme type II superconductors. Universal properties and trends," Physica, 207, 366-380 (1993).

290. F. a recent analysis of the doping dependence of $\xi_{0}$ see Y. Ando and K. Segawa, "Magnetoresistance of untwinned $\mathrm{YBa}_{2} \mathrm{Cu}_{3} \mathrm{O}_{y}$ single crystals in a wide range of doping: anomalous hole-doping dependence of the coherence length," Phys. Rev. Lett., 88, 167005 (2002).

291. C. P. B. W. N. Hardy, D. A. Bonn, and R. Liang, "Magnetic field dependence of lambda in $\mathrm{YBa}_{2} \mathrm{Cu}_{3} \mathrm{O}_{6.95}$ : results as a function of temperature and field orientation," Phys. Rev. Lett., 83, 3277-3280 (1999).

292. X.-G. Wen and P. A. Lee, "Theory of quasiparticles in the underdoped high-T ${ }_{c}$ superconducting state," Phys. Rev. Lett., 80, 2193-2196 (1998).

293. A. J. Millis, S. M. Girvin, L. B. Ioffe, and A. I. Larkin, "Anomalous charge dynamics in the superconducting state of underdoped cuprates," Jour. Phys. Chem. Solids, 59, 1742-1744 (1998).

294. G. T. Zimanyi, S. A. Kivelson, and A. Luther, "Superconductivity from predominantly repulsive interactions in quasi one-dimensional systems," Phys. Rev. Lett., 60, 2089-2092 (1988).

295. S. A. Kivelson and G. T. Zimanyi, "High temperature superconductors, RVB, and conducting polymers," Molec. Cryst. and Liq. Cryst, 160, 457-481 (1988).

296. A. M. Finkel'stein and S. A. Brazovsky, "Interchain coupling in linear conductors," J. Phys. C, 14, 847-857 (1981).

297. S. A. Brazovsky and A. M. Finkel'stein, "The influence of phonons on the optical properties and conductivity of quasi-one-dimensional metals," Solid State Comm., 38, 745 (1981).

298. S. A. Kivelson and M. I. Salkola, "Metal-non-metal transition in polyacetylene," Synth. Met., 44, 281-291 (1991).

299. G. G. Batrouni, R. T. Scalettar, G. T. Zimanyi, and A. P. Kampf, "Supersolids in the Bose-Hubbard Hamiltonian," Phys. Rev. Lett., 74, 2527-2530 (1995), see also 498.

300. M. P. A. Fisher, P. B. Weichman, G. Grinstein, and D. S. Fisher, "Boson localization and the superfluid-insulator transition," Phys. Rev. B, 40, 546570 (1989). 
301. C. Nayak and K. Shtengel, "Microscopic models of two-dimensional magnets with fractionalized excitations," Phys. Rev. B, 64, 064422-064428 (2001).

302. R. Coldea, D. A. Tennant, A. M. Tsvelik, and Z. Tylczynski, "Experimental realization of a 2D fractional quantum spin liquid," Phys. Rev. Lett., 86, 1335-1338 (2001).

303. S. Chakravarty, "Magnetic properties of $\mathrm{La}_{2} \mathrm{CuO}_{4}$," (Addison-Wesley, Redwood City, CA, USA) (1990).

304. A. E. Sikkema, I. Affleck, and S. R. White, "Spin gap in a doped Kondo chain," Phys. Rev. Lett., 79, 929-932 (1997).

305. O. Zachar, V. J. Emery, and S. A. Kivelson, "Exact results for a 1D Kondo lattice from bosonization," Phys. Rev. Lett., 77, 1342-1345 (1996).

306. O. Zachar, "Staggered liquid phases of the one-dimensional Kondo-Heisenberg lattice model," Phys. Rev. B, 63, 205104-205113 (2001).

307. D. J. Scalapino and S. R. White, "Numerical results for the Hubbard model: implications for the high $\mathrm{T}_{c}$ pairing mechanism," Foundations of Physics, 31, 27-39 (2001).

308. F. D. M. Haldane, "Continuum dynamics of the 1-D Heisenberg antiferromagnet: identification with the $\mathrm{O}(3)$ nonlinear sigma model," Phys. Lett. A, 93, 464-468 (1983).

309. S. Chakravarty, "Dimensional crossover in quantum antiferromagnets," Phys. Rev. Lett., 77, 4446-4449 (1996).

310. S. R. White, R. M. Noack, and D. J. Scalapino, "Resonating valence bond theory of coupled Heisenberg chains," Phys. Rev. Lett., 73, 886-889 (1994).

311. D. J. Scalapino and S. A. Trugman, "Local antiferromagnetic correlations and $d_{x^{2}-y^{2}}$ pairing," Philos. Mag. B, 74, 607-610 (1996).

312. M. Havilio and A. Auerbach, "Superconductivity and quantum spin disorder in cuprates," Phys. Rev. Lett., 83, 4848-4851 (1999).

313. M. Havilio and A. Auerbach, "Correlations in doped antiferromagnets," Phys. Rev. B, 62, 324-336 (2000).

314. S. R. White and D. J. Scalapino, "Superconductivity in ladders and coupled planes," Phys. Rev. B, 45, 5744-5747 (1992).

315. D. Poilblanc, "Internal structure of the singlet $d_{x^{2}-y^{2}}$ hole pair in an antiferromagnet," Phys. Rev. B, 495, 1477-1479 (1993).

316. Y. Fang, A. E. Ruckenstein, E. Dagotto, and S. Schmitt-Rink, "Holes in the infinite-U Hubbard model: instability of the Nagaoka state," Phys. Rev. B, 40, 7406-7409 (1989), see also [499].

317. D. Rokhsar, "Quadratic quantum antiferromagnets in the fermionic large-N limit," Phys. Rev. B, 42, 2526-2531 (1990).

318. C. M. Varma and A. Zawadowskii, "Scaling in an interacting two-component (valence-fluctuation) electron gas," Phys. Rev. B, 32, 7399-7407 (1985).

319. H.-H. Lin, L. Balents, and M. P. A. Fisher, "N-chain Hubbard model in weak coupling," Phys. Rev. B, 56, 6569-6593 (1997).

320. S. R. White and D. J. Scalapino, "Density matrix renormalization group study of the striped phase in the 2D t-J model," Phys. Rev. Lett., 80, 1272-1275 (1998).

321. S. R. White and D. J. Scalapino, "Energetics of domain walls in the 2D t-J model," Phys. Rev. Lett., 81, 3227-3230 (1998).

322. S. Hellberg and E. Manousakis, "Phase separation at all interaction strengths in the t-J model," Phys. Rev. Lett., 78, 4609-4612 (1997). 
323. D. Poilblanc, O. Chiappa, J. Riera, S. R. White, and D. J. Scalapino, "Evolution of the spin gap upon doping a 2-leg ladder," Phys. Rev. B, 62, R14633R14636 (2000).

324. S. R. White and D. J. Scalapino, "Competition between stripes and pairing in a t-t'-J model," Phys. Rev. B, 60, R753-R756 (1999).

325. S. C. Hellberg and E. Manousakis, "Stripes and the t-J model," Phys. Rev. Lett., 83, 132 (1999), see also Refs. 326, 327.

326. S. R. White and D. J. Scalapino, "Comment on "Stripes and the t-J Model"," Phys. Rev. Lett., 84, 3021 (2000).

327. S. C. Hellberg and E. Manousakis, "Reply: Hellberg and Manousakis," Phys. Rev. Lett., 84, 3022 (2000).

328. E. Arrigoni, A. P. Harju, W. Hanke, B. Brendel, and S. A. Kivelson, "Stripes and superconducting pairing in the t-J model with Coulomb interactions," Phys. Rev. B, 65, 134503-134507 (2002).

329. S. R. White and D. J. Scalapino, "Phase separation and stripe formation in the two-dimensional t-J model: A comparison of numerical results," Phys. Rev. B, 61, 6320-6326 (2000).

330. E. Daggoto, "Correlated electrons in high-temperature superconductors," Rev. Mod. Phys., 66, 763-840 (1994).

331. S. C. Hellberg and E. Manousakis, "Green's-function Monte Carlo for lattice fermions: Application to the t-J model," Phys. Rev. B, 61, 11787-11806 (2000).

332. S. Sorella, "Green function Monte Carlo with stochastic reconfiguration," Phys. Rev. Lett., 80, 4558-4561 (1998).

333. Y. C. Chen and T. K. Lee, "t-J model studied by the power Lanczos method," Phys. Rev. B, 51, 6723-6726 (1995).

334. S. R. White, "Density-matrix algorithms for quantum renormalization groups," Phys. Rev. B, 48, 10345-10356 (1993).

335. S. R. White, "Spin gaps in a frustrated Heisenberg model for $\mathrm{CaV}_{4} \mathrm{O}_{9}$," Phys. Rev. Lett., 77, 3633-3636 (1996).

336. E. Dagotto and T. M. Rice, "Surprises on the way from one- to twodimensional quantum magnets: the ladder materials," Science, 271, 618-623 (1996).

337. E. Dagotto, "Experiments on ladders reveal a complex interplay between a spin-gapped normal state and superconductivity," Rep. Prog. Phys., 62, 15251571 (1999).

338. E. Lieb and F. Y. Wu, "Absence of Mott transition in an exact solution of the short-range, one-band model in one dimension," Phys. Rev. Lett., 20, 14451448 (1968).

339. H. J. Schulz, in "Proceedings of the 9th Jerusalem Winter School for Theoretical Physics," edited by V. J. Emery (World Scientific, New York) (1993).

340. B. Frischmuth, B. Ammon, and M. Troyer, "Susceptibility and lowtemperature thermodynamics of spin-1/2 Heisenberg ladders," Phys. Rev. B, 54, R3714-R3717 (1996).

341. R. M. Noack, S. R. White, and D. J. Scalapino, "Correlations in a two-chain Hubbard model," Phys. Rev. Lett., 73, 882-885 (1994).

342. R. M. Noack, D. J. Scalapino, and S. R. White, "The ground state of the twoleg Hubbard ladder. A density-matrix renormalization group study," Physica $C, \mathbf{2 7 0}, 281-296$ (1996). 
343. E. Dagotto, J. Riera, and D. Scalapino, "Superconductivity in ladders and coupled planes," Phys. Rev. B, 45, 5744-5747 (1992).

344. T. Kimura, K. Kuroki, and H. Aoki, "Pairing correlation in the three-leg Hubbard ladder-renormalization group and quantum Monte Carlo studies," J. Phys. Soc. Jpn., 67, 1377-1390 (1998).

345. E. Jeckelmann, D. J. Scalapino, and S. R. White, "Comparison of different ladder models," Phys. Rev. B, 58, 9492-9497 (1998).

346. S. Daul, D. J. Scalapino, and S. R.White, "Pairing correlations on t-U-J ladders," Phys. Rev. Lett., 84, 4188-4191 (2000).

347. S. R. White and D. J. Scalapino, "Ground-state properties of the doped threeleg t-J ladder," Phys. Rev. B, 57, 3031-3037 (1998).

348. T. M. Rice, S. Haas, M. Sigrist, and F.-C. Zhang, "Lightly doped t-J three-leg ladders: An analog for the underdoped cuprates," Phys. Rev. B, 56, 1465514667 (1997).

349. W. R. White and D. J. Scalapino, "Ground states of the doped four-leg t-J ladder," Phys. Rev. B, 55, R14701-R14704 (1997).

350. D. J. Scalapino, private communication.

351. M. Ogata, M. U. Luchini, S. Sorella, and F. F. Assaad, "Phase diagram of the one-dimensional t-J model," Phys. Rev. Lett., 66, 2388-2391 (1991).

352. C. S. Hellberg and E. J. Mele, "Luttinger-liquid instability in the onedimensional t-J model," Phys. Rev. B , 48, 646-649 (1993).

353. M. Troyer, H. Tsunetsugu, and T. . M. Rice, "Properties of lightly doped t-J two-leg ladders," Phys. Rev. B, 53, 251-267 (1996).

354. C. A. Hayward and D. Poilblanc, "Luttinger-liquid behavior and superconducting correlations in t-J ladders," Phys. Rev. B, 53, 11721-11728 (1996).

355. G. Sierra, M. A. Martin-Delgado, J. Dukelsky, S. R. White, and D. J. Scalapino, "Dimer-hole-RVB state of the two-leg t-J ladder: A recurrent variational ansatz," Phys. Rev. B, 57, 11666-11673 (1998).

356. S. Rommer, S. R. White, and D. J. Scalapino, "Phase separation in t-J ladders," Phys. Rev. B, 61, 13424-13430 (2000).

357. S. R. White, I. Affleck, and D. J. Scalapino, "Friedel oscillations and charge density waves in chains and ladders," Phys. Rev. B, 65, 165122-165134 (2002).

358. S. A. Kivelson and V. J. Emery, "Strongly Correlated Electronic Materials: The Los Alamos Symposium 1993," (Addison-Wesley, Redwood City, CA, USA) (1994).

359. A. C. Cosentini, M. Capone, L. Guidoni, and G. B. Bachelet, "Phase separation in the two-dimensional Hubbard model: A fixed-node quantum Monte Carlo study," Phys. Rev. B, 58, R14685-R14688 (1998).

360. F. Becca, M. Capone, and S. Sorella, "Spatially homogeneous ground state of the two-dimensional Hubbard model," Phys. Rev. B, 62, 12700-12706 (2001).

361. S. Hellberg and E. Manousakis, "2-Dimensional t-J Model at Low Electron Density," Phys. Rev. B, 52, 4639-4642 (1995).

362. V. J. Emery, S. A. Kivelson, and H. Q. Lin, "Phase separation in the t-J model," Phys. Rev. Lett., 64, 475-478 (1990).

363. S. A. Kivelson, V. J. Emery, and H. Q. Lin, "Doped antiferromagnets in the weak-hopping limit," Phys. Rev. B, 42, 6523-6530 (1990).

364. E. Eisenberg, R. Berkovits, D. A. Huse, and B. L. Altshuler, "The breakdown of the Nagaoka phase in the 2D t-J model," Phys. Rev. B, 65, 134437-134443 (2002). 
365. W. O. Putikka, M. U. Luchini, and T. M. Rice, "Aspects of the phase diagram of the two-dimensional t-J model," Phys. Rev. Lett., 68, 538-541 (1992).

366. D. Poilblanc, "Phase diagram of the two-dimensional t-J model at low doping," Phys. Rev. B, 52, 9201-9204 (1995).

367. M. Calandra, F. Becca, and S. Sorella, "Charge fluctuations close to phase separation in the two-dimensional t-J model," Phys. Rev. Lett., 81, 5185-5188 (1998).

368. H. Yokoyama and M. Ogata, "Phase diagram and pairing symmetry of the two-dimensional t-J model by a variation theory," J. Phys. Soc. Japan, 65, 3615-3629 (1996).

369. C. T. Shih, Y. C. Chen, and T. K. Lee, "Phase separation of the twodimensional t-J model," Phys. Rev. B, 57, 627-631 (1998).

370. C. S. Shin, Y. C. Chen, and T. K. Lee, "Revisit phase separation of the twodimensional $t-J$ model by the Power-Lanczos method," cond-mat/0104067 (2001).

371. M. Khono, "Ground-state properties of the two-dimensional t-J model," Phys. Rev. B, 55, 1435-1441 (1997).

372. F. Becca, L. Capriotti, and S. Sorella, "Stripes and spin incommensurabilities are favored by lattice anisotropies," Phys. Rev. Lett., 87, 167005-167008 (2001).

373. S. R. White and D. J. Scalapino, "Why do stripes form in doped antiferromagnets and what is their relationship to superconductivity?" cond-mat/0006071 (2000).

374. C. Nayak and F. Wilczek, "Possible electronic structure of domain walls in Mott insulators," Int. J. Mod. Phys. B, 10, 2125-2136 (1996).

375. J. Zaanen and O. Gunnarsson, "Charged magnetic domain lines and the mag-

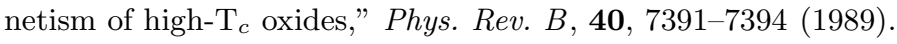

376. D. Poilblanc and T. M. Rice, "Charged solitons in the Hartree-Fock approximation to the large-U Hubbard model," Phys. Rev. B, 39, 9749-9752 (1989).

377. H. J. Schulz, "Domain walls in a doped antiferromagnet," J. de Physique, 50, 2833-2849 (1989).

378. K. Machida, "Magnetism in $\mathrm{La}_{2} \mathrm{CuO}_{4}$ based compounds," Physica $C$, 158, 192-196 (1989).

379. O. Zachar, "Stripes: Why hole rich lines are antiphase domain walls?" condmat/0001217 (2000).

380. W. V. Liu and E. Fradkin, "Antiferromagnetic spin ladders effectively coupled by one-dimensional electron liquids," Phys. Rev. Lett., 86, 1865-1868 (2001).

381. A. L. Chernyshev, S. R. White, and A. H. Castro Neto, "Charge stripe in an antiferromagnet: 1d band of composite excitations," cond-mat/0111474 (2001), see also Ref. 408 .

382. A. Moreo, "Pairing correlations in the two-dimensional Hubbard model," Phys. Rev. B, 45, 5059-5061 (1992).

383. S.-C. Zhang, J. Carlson, and J. E. Gubernatis, "Constrained path Monte Carlo method for fermion ground states," Phys. Rev. B, 55, 7464-7477 (1997).

384. E. Dagotto and J. Riera, "Indications of $d_{2^{x}-y^{2}}$ superconductivity in the two dimensional t-J model," Phys. Rev. Lett., 70, 682-685 (1993).

385. E. Dagotto, A. Moreo, F. Ortolani, D. Poilblanc, and J. Riera, "Static and dynamical properties of doped Hubbard clusters," Phys. Rev. B, 45, 1074110760 (1992). 
386. M. Calandra and S. Sorella, "From antiferromagnetism to d-wave superconductivity in the two-dimensional t-J model," Phys. Rev. B, 61, R11894R11897 (2000).

387. S. Sorella, G. B. Martins, F. Becca, C. Gazza, L. Capriotti, A. Parola, and E. Dagotto, "Superconductivity in the two-dimensional t-J model," condmat/0110460 (2001).

388. T. Tohyama, C. G. C. T. Shih, Y. C. Chen, T. K. Lee, S. Maekawa, and E. Dagotto, "Stripe stability in the extended t-J model on planes and four-leg ladders," Phys. Rev. B, 59, R11649-R11652 (1999).

389. E. L. Nagaev, Physics of Magnetic Semiconductors (Mir, Moscow) (1983).

390. A. Auerbach and B. E. Larson, "Doped antiferromagnet: The instability of homogeneous magnetic phases," Phys. Rev. B, 43, 7800-7809 (1991).

391. A. Auerbach and B. E. Larson, "Small-polaron theory of doped antiferromagnets," Phys. Rev. Lett., 66, 2262-2265 (1990).

392. A. Auerbach, "Spin tunneling, Berry phases, and doped antiferromagnets," Phys. Rev. B, 48, 3287-3289 (1993).

393. E. W. Carlson, S. A. Kivelson, Z. Nussinov, and V. J. Emery, "Doped antiferromagnets in high dimension," Phys. Rev. B, 57, 14704-14721 (1998).

394. P. B. Visscher, "Phase separation instability in the Hubbard model," Phys. Rev. B, 10, 943-945 (1974).

395. L. B. Ioffe and A. I. Larkin, "Two-dimensional Hubbard model with strong electron repulsion," Phys. Rev. B, 37, 5730-5737 (1988).

396. L. Pryadko, D. Hone, and S. A. Kivelson, "Instability of charge ordered states in doped antiferromagnets," Phys. Rev. Lett., 80, 5651-5654 (1998).

397. J. K. Freericks, E. H. Lieb, and D. Ueltschi, "Segregation in the FalicovKimball model," math-ph/0107003 (2001).

398. E. L. Nagaev, "Lanthanum manganites and other giant-magnetoresistance magnetic conductors," Usp. Fiz. Nauk., 166, 833 (1996).

399. S. Trugman, "Interaction of holes in a Hubbard antiferromagnet and hightemperature superconductivity," Phys. Rev. B, 37, 1597-1603 (1988).

400. A. H. Castro Neto, "Landau theory of phase separation in cuprates," Phys. Rev. B, 51, 3254-3256 (1995).

401. M. Seul and D. Andelman, "Domain shapes and patterns - the phenomenology of modulated phases," Science, 267, 476-483 (1995).

402. B. P. Stojkovic, Z. G. Yu, A. R. Bishop, A. H. Castro Neto, and N. GronbechJensen, "Charge ordering and long-range interactions in layered transition metal oxides," Phys. Rev. Lett., 82, 4679-4682 (1999).

403. L. P. Pryadko, S. A. K. V. J. Emery, Y. B. Bazaliy, and E. A. Demler, "Topological doping and the stability of stripe phases," Phys. Rev. B, 60, 7541-7557 (1999).

404. A. L. Chernyshev, A. H. Castro Neto, and A. R. Bishop, "Metallic stripe in two dimensions: stability and spin-charge separation," Phys. Rev. Lett., 84, 4922-4925 (2000).

405. S. A. Kivelson and V. J. Emery, "Topological doping of correlated insulators," Synthetic Metals, 80, 151-158 (1996).

406. J. Zaanen and Z. Nussinov, "Stripes and nodal fermions as two sides of the same coin," cond-mat/0006193 (2000).

407. J. Han, Q. H. Wang, and D. H. Lee, "Antiferromagnetism, stripes, and superconductivity in the t-J model with Coulomb interaction," Int. J. Mod. Phys. B, 15, 1117-1126 (2001). 
408. A. H. Castro Neto, "Luttinger stripes in antiferromagnets," Z. Phys. B, 103, 185-192 (1997).

409. J. Zaanen and P. B. Littlewood, "Freezing Electronic Correlations by Polaronic Instabilities in Doped $\mathrm{La}_{2} \mathrm{NiO}$," Phys. Rev. B, 50, 7222-7225 (1994).

410. H. J. Schulz, "Incommensurate antiferromagnetism in the two-dimensional Hubbard model," Phys. Rev. Lett., 64, 1445-1448 (1990).

411. A. I. Larkin, "Effect of inhomogeneities on the structure of the mixed state of superconductors," Sov. Phys. JETP, 31, 784-786 (1970).

412. V. J. Emery and S. A. Kivelson, "Charge inhomogeneity and high temperature superconductivity," J. Phys. Chem. Sol., 61, 467-471 (2000).

413. N. Ichikawa, S. Uchida, J. M. Tranquada, T. Niemoller, P. M. Gehring, S. H. Lee, and J. R. Schneider, "Local magnetic order vs superconductivity in a layered cuprate," Phys. Rev. Lett., 85, 1738-1741 (2000).

414. J. Schmalian and P. G. Wolynes, "Stripe glasses: Self-generated randomness in a uniformly frustrated system," Phys. Rev. Lett., 85, 836-839 (2000).

415. J. Burgy, M. Mayr, V. Martin-Mayor, A. Moreo, and E. Dagotto, "Colossal effects in transition metal oxides caused by intrinsic inhomogeneities," Phys. Rev. Lett., 87, 277202-277205 (2001).

416. U. Löw, V. J. Emery, K. Fabricius, and S. A. Kivelson, "Study of an Ising model with competing long- and short-range interactions," Phys. Rev. Lett., 72, 1918-1921 (1994).

417. M. Grousson, G. Tarjus, and P. Viot, "Monte Carlo study of the threedimensional Coulomb frustrated Ising ferromagnet," Phys. Rev. E, 64, 036109-036117 (2001).

418. L. Chayes, V. J. Emery, S. A. Kivelson, Z. Nussinov, and G. Tarjus, "Avoided critical behavior in a uniformly frustrated system," Physica A, 225, 129-153 (1996).

419. Z. Nussinov, J. Rudnick, S. A. Kivelson, and L. N. Chayes, "Avoided critical behavior in O(n) systems," Phys. Rev. Lett., 83, 472-475 (1999).

420. D. B. Tanner and T. Timusk, "Optical properties of high-temperature superconductors," in "Physical Properties of High Temperature Superconductors Vol. III," edited by D. M. Ginsberg (World Scientific, Singapore) (1992).

421. N. P. Ong, "Transport properties of high $T_{c}$ cuprates," in "Physical Properties of High Temperature Superconductors Vol. II," edited by D. M. Ginsberg (World Scientific, Singapore) (1992).

422. K. Krishana, J. M. Harris, and N. P. Ong, "Quasiparticle mean free path in $\mathrm{YBa}_{2} \mathrm{Cu}_{3} \mathrm{O}_{7}$ measured by the thermal Hall conductivity," Phys. Rev. Lett., 75, 3529-3532 (1995).

423. K. M. Lang, V. Madhavan, J. E. Hoffman, E. W. Hudson, H. Eisaki, S. Uchida, and J. C. Davis, "Imaging the granular structure of high- $\mathrm{T}_{c}$ superconductivity in underdoped $\mathrm{Bi}_{2} \mathrm{Sr}_{2} \mathrm{CaCu}_{2} \mathrm{O}_{8+\text { delta }}$," Nature, 415, 412-416 (2002).

424. T. R. Thurston, R. J. Birgeneau, M. A. Kastner, N. W. Preyer, G. Shirane, Y. Fujii, K. Yamada, Y. Endoh, K. Kakurai, M. Matsuda, Y. Hidaka, and T. Murakami, "Neutron scattering study of the magnetic excitations in metallic and superconducting $\mathrm{La}_{2-x} \mathrm{Sr}_{x} \mathrm{CuO}_{4-y}$," Phys. Rev. B, 40, 45854595 (1989).

425. S.-W. Cheong, G. Aeppli, T. E. Mason, H. Mook, S. M. Hayden, P. C. Canfield, Z. Fisk, K. N. Clausen, and J. L. Martinez, "Incommensurate magnetic fluctuations in $\mathrm{La}_{2-x} \mathrm{Sr}_{x} \mathrm{CuO}_{4}$," Phys. Rev. Lett., 67, 1791-1794 (1991). 
426. J. M. Tranquada, B. J. Sternlieb, J. D. Axe, Y. Nakamura, and S. Uchida, "Evidence for stripe correlations of spins and holes in copper oxide superconductors," Nature, 375, 561-563 (1995).

427. J. M. Tranquada, D. J. Buttrey, V. Sachan, and J. E. Lorenzo, "Simultaneous ordering of holes and spins in $\mathrm{La}_{2} \mathrm{NiO}_{4.125}$," Phys. Rev. Lett., 73, 1003-1006 (1994).

428. A. Lanzara, P. V. Bogdanov, X. J. Zhou, S. A. Kellar, D. L. Feng, E. D. Lu, T. Yoshida, H. Eisaki, A. Fujimori, K. Kishio, J.-I. Shimoyama, T. Noda, S. Uchida, Z. Hussain, and Z.-X. Shen, "Evidence for ubiquitous strong electron-phonon coupling in high-temperature superconductors," Nature, 412, 510-514 (2001).

429. L. P. Gor'kov and A. V. Sokol, "On the problem of the phase diagram of new superconductors," J. Physique, 50, 2823-32 (1989).

430. A. J. Heeger, S. A. Kivelson, J. R. Schrieffer, and W. Su, "Solitons in conducting polymers," Rev. Mod. Phys., 60, 781-850 (1988).

431. D. K. Campbell, A. R. Bishop, and K. Fesser, "Polarons in quasi-onedimensional systems," Phys. Rev. B, 26, 6862-6874 (1982).

432. L. B. Ioffe and A. Larkin, "Superconductivity in the liquid-dimer valence-bond state," Phys. Rev. B, 40, 6941-6947 (1989).

433. M. Marder, N. Papanicolaou, and G. C. Psaltakis, "Phase separation in a t-J model," Phys. Rev. B, 41, 6920-32 (1990).

434. M. Vojta and S. Sachdev, "Charge order, superconductivity and a global phase diagram of doped antiferromagnets," Phys. Rev. Lett., 83, 3916-3919 (1999).

435. S. Sachdev and S.-C. Zhang, "Tuning order in cuprate superconductors," Science, 295, 452 (2002).

436. D. I. Khomskii and K. I. Kugel, "Why stripes? Spontaneous formation of inhomogeneous structures due to elastic interactions," Europhys. Lett., 55, 208-213 (2001).

437. A. A. Koulakov, M. M. Fogler, and B. I. Shklovskii, "Charge density wave in two-dimensional electron liquid in weak magnetic field," Phys. Rev. Lett., 76, 499-502 (1996).

438. R. Moessner and T. J. Chalker, "Exact results for interacting electrons in high Landau levels," Phys. Rev. B, 54, 5006-5015 (1996).

439. M. P. Lilly, K. B. Cooper, J. P. Eisenstein, L. N. Pfeiffer, and K. W. West, "Evidence for an anisotropic state of two-dimensional electrons in high Landau levels," Phys. Rev. Lett., 82, 394-397 (1999).

440. B. Spivak, "Title: Phase separation in the two-dimensional electron liquid in MOSFETs," cond-mat/0205127 (2002).

441. S. A. Kivelson, "Making high $\mathrm{T}_{c}$ higher: a theoretical proposal," Physica B, 61-67, 61-67 (2002).

442. T. H. Geballe and B. Y. Moyzhes, "Qualitative understanding of the highest Tc cuprates," Physica C, 342, 1821-1824 (2000).

443. M. Fujita, H. Goka, K. Yamada, and M. Matsuda, "Competition between charge/spin-density-wave orders and superconductivity in $\mathrm{La}_{1.875} \mathrm{Ba}_{0.125-x} \mathrm{Sr}_{x} \mathrm{CuO}_{4}$," Phys. Rev. Lett., 88, 167008-167011 (2002).

444. K. Yamada, C. H. Lee, K. Kurahashi, J. Wada, S. Wakimoto, S. Ueki, H. Kimura, Y. Endoh, S. Hosoya, G. Shirane, R. J. Birgeneau, M. Greven, M. A. Kastner, and Y. J. Kim, "Doping dependence of the spatially modulated dynamical spin correlations and the superconducting-transition temperature in $\mathrm{La}_{2-x} \mathrm{Sr}_{x} \mathrm{CuO}_{4}, "$ Phys. Rev. B, 57, 6165-6172 (1998). 
445. Y. S. Lee, R. J. Birgeneau, M. A. Kastner, Y. Endoh, S. Wakimoto, K. Yamada, R. W. Erwin, S.-H. Lee, and G. Shirane, "Neutron-scattering study of spin-density wave order in the superconducting state of excess-oxygen-doped $\mathrm{La}_{2} \mathrm{CuO}_{4+y}$," Phys. Rev. B, 60, 3643-3654 (1999).

446. B. Khaykovich, Y. S. Lee, R. Erwin, S.-H. Lee, S. Wakimoto, K. J. Thomas, M. A. Kastner, and R. J. Birgeneau, "Enhancement of long-range magnetic order by magnetic field in superconducting $\mathrm{La}_{2} \mathrm{CuO}_{4+}$," cond-mat/0112505 (2001).

447. H. Kimura, K. Hirota, H. Matsushita, K. Yamada, Y. Endoh, S.-H. Lee, C. F. Majkrzak, R. Erwin, G. Shirane, M. Greven, Y. S. Lee, M. A. Kastner, and R. J. Birgeneau, "Neutron-scattering study of static antiferromagnetic correlations in $\mathrm{La}_{2-x} \mathrm{Sr}_{x} \mathrm{Cu}_{1-y} \mathrm{Zn}_{y} \mathrm{O}_{4}$," Phys. Rev. B, 59, 6517-6523 (1999).

448. M. Fujita, K. Yamada, H. Hiraka, P. M. Gehring, S. H. Lee, S. Wakimoto, and G. Shirane, "Static magnetic correlations near the insulating-superconducting phase boundary in $\mathrm{La}_{2-x} \mathrm{Sr}_{x} \mathrm{CuO}_{4}$, , Phys. Rev. B, 65, 064505-064511 (2002).

449. P. Dai, H. A. Mook, R. D. Hunt, and F. Dogan, "Evolution of the resonance and incommensurate spin fluctuations in superconducting $\mathrm{YBa}_{2} \mathrm{Cu}_{3} \mathrm{O}_{6+x}$," Phys. Rev. B, 63, 054525-054544 (2001).

450. P. Bourges, Y. Sidis, H. F. Fong, L. P. Regnault, J. Bossy, A. Ivanov, and B. Keimer, "The spin excitation spectrum in superconducting $\mathrm{YBa}_{2} \mathrm{Cu}_{3} \mathrm{O}_{6.85}, "$ Science, 288, 1234-1237 (2000).

451. G. Aeppli, S. M. Hayden, P. Dai, H. A. Mook, R. D. Hunt, T. G. Perring, and F. Dogan, "The weights of various features in the magnetic spectra of cuprates," Phys. Stat. Sol., 215, 519-522 (1999).

452. H. A. Mook, P. C. Dai, F. Dogan, and R. D. Hunt, "One-dimensional nature of the magnetic fluctuations in $\mathrm{YBa}_{2} \mathrm{Cu}_{3} \mathrm{O}_{6}$, , Nature, 404, 729-731 (2000).

453. H. A. Mook and B. C. Chakoumakos, "Incommensurate fluctuations in $\mathrm{Bi}_{2} \mathrm{Sr}_{2} \mathrm{CaCu}_{2} \mathrm{O}_{8}$," Jour. Superconductivity, 10, 389-392 (1997).

454. J. E. Hoffman, E. W. Hudson, K. M. Lang, V. Madhavan, H. Eisaki, S. Uchida, and J. C. Davis, "A four unit cell periodic pattern of quasi-particle states surrounding vortex cores in $\mathrm{Bi}_{2} \mathrm{Sr}_{2} \mathrm{CaCu}_{2} \mathrm{O}_{8+x}$," Science, 295, 466-469 (2002).

455. C. Howald, H. Eisaki, N. Kaneko, and A. Kapitulnik, "Coexistence of charged stripes and superconductivity in $\mathrm{Bi}_{2} \mathrm{Sr}_{2} \mathrm{CaCu}_{2} \mathrm{O}_{8+\delta}$," cond-mat/0201546.

456. J. E. Hoffman, K. McElroy, D.-H. Lee, K. M. Lang, H. Eisaki, S. Uchida, and J. C. Davis, "Imaging quasiparticle quantum interference in BSCCO," submitted to Science (2002).

457. E. Fradkin, S. A. Kivelson, E. Manousakis, and K. Nho, "Nematic phase of the two-dimensional electron gas in a magnetic field," Phys. Rev. Lett., 84, 1982-1985 (2000).

458. J. Zaanen, M. L. Horbach, and W. V. Saarloos, "Charged domain-wall dynamics in doped antiferromagnets and spin fluctuations in cuprate superconductors," Phys. Rev. B, 53, 8671-8680 (1996).

459. V. Oganesyan, S. A. Kivelson, and E. Fradkin, "Quantum theory of a nematic Fermi fluid," Phys. Rev. B, 64, 195109-195114 (2001).

460. C. Halboth and W. Metzner, "d-wave superconductivity and Pomeranchuk instability in the two-dimensional Hubbard model," Phys. Rev. Lett., 85, 51625165 (2000).

461. V. Hankevych, I. Grote, and F. Wegner, "Pomeranchuk and other instabilities in the $t-t^{\prime}$ Hubbard model at the Van Hove filling," cond-mat/0205213 (2002). 
462. V. Oganesyan, E. Fradkin, and S. A. Kivelson, work in progress (2002).

463. A. Abanov, V. Kalatsky, and V. L. Pokrovsky, "Phase diagram of ultrathin ferromagnetic films with perpendicular anisotropy," Phys. Rev. B, 51, 10231038 (1995).

464. J. Zaanen, O. Y. Osman, H. V. Kruis, Z. Nussinov, and J. Tworzydlo, "The geometric order of stripes and Luttinger liquids," Phil. Mag. B, 81, 1485-1531 (2002).

465. Y.-B. Kim and H.-Y. Kee, "Pairing instability in a nematic Fermi liquid," cond-mat/0204037 (2002).

466. K. B. Cooper, M. Lilly, J. P. Eisenstein, L. N. Pfeiffer, and K. W. West, "The onset of anisotropic transport of two-dimensional electrons in high Landau levels: An isotropic-to-nematic liquid crystal phase transition?" condmat/0203174 (2002).

467. V. J. Emery and S. Kivelson, "Mapping of the two-channel Kondo problem to a resonant-level model," Phys. Rev. B, 46, 10812-10817 (1992).

468. V. J. Emery and S. A. Kivelson, "Solution of an orbital Kondo array," Phys. Rev. Lett., 71, 3701-3704 (1993).

469. D. Chang and D. H. Lee, "Transport in inhomogeneous strongly correlated systems," cond-mat/0205057 (2002).

470. Z. M. Yusof, B. O. Wells, T. Valla, A. V. Fedorov, P. D. Johnson, Q. Li, C. Kendziora, S. Jian, and D. G. Hinks, "Quasiparticle liquid in the highly overdoped $\mathrm{Bi}_{2} \mathrm{Sr}_{2} \mathrm{CaCu}_{2} \mathrm{O}_{8+\delta}$," Phys. Rev. Lett., 88, 167006-167009 (2002).

471. M. Granath, V. Oganesyan, D. Orgad, and S. A. Kivelson, "Distribution of spectral weight in a system with disordered stripes," Phys. Rev. B, 65, 184501-184510 (2002).

472. M. I. Salkola, V. J. Emery, and S. A. Kivelson, "Implications of charge ordering for single-particle properties of high $T_{c}$ superconductors," Phys. Rev. Lett., 77, 155-158 (1996).

473. M. G. Zacher, R. Eder, E. Arrigoni, and W. Hanke, "Stripes in doped antiferromagnets: single-particle spectral weight," Phys. Rev. Lett., 85, 2585-2588 (2000).

474. M. G. Zacher, R. Eder, E. Arrigoni, and W. Hanke, "Evolution of the stripe phase as a function of doping from a theoretical analysis of angle-resolved photoemission data," Phys. Rev. B, 65, 045109-045117 (2002).

475. M. Vojta, Y. Zhang, and S. Sachdev, "Renormalization group analysis of quantum critical points in d-wave superconductors," Int. J. Mod. Phys.B, 14, 3719-3734 (2000).

476. T. Valla, A. V. Fedorov, P. D. Johnson, B. O. Wells, S. L. Hulbert, Q. Li, G. D. Gu, and N. Koshizuka, "Evidence for quantum critical behavior in the optimally doped cuprate $\mathrm{Bi}_{2} \mathrm{Sr}_{2} \mathrm{CaCu}_{2} \mathrm{O}_{8}$," Science, 285, 2110-2113 (1999).

477. Y. Zhang, N. P. Ong, P. W. Anderson, D. A. Bonn, R. Liang, and W. N. Hardy, "Giant enhancement of the thermal Hall conductivity kappa(xy) in the superconductor $\mathrm{YBa}_{2} \mathrm{Cu}_{3} \mathrm{O}_{7}$," Phys. Rev. Lett., 86, 890-893 (2001).

478. D. A. Bonn, P. Dosanjh, R. Liang, and W. N. Hardy, "Evidence for rapid suppression of quasi-particle scattering below $\mathrm{T}_{c}$ in $\mathrm{YBa}_{2} \mathrm{Cu}_{3} \mathrm{O}_{7}$," Phys. Rev. Lett., 68, 2390-2393 (1992), see also Ref. 500.

479. H. F. Fong, P. Bourges, Y. Sidis, L. P. Regnault, A. Ivanov, G. D. Gul, N. Koshizuka, and B. Keimer, "Neutron scattering from magnetic excitation in $\mathrm{Bi}_{2} \mathrm{Sr}_{2} \mathrm{CaCu}_{2} \mathrm{O}_{8+\delta}$," Nature, 398, 588-591 (1999). 
480. M. Dumm, D. N. Basov, S. Komiya, and Y. Ando, "Anistropic electromagnetic response of $\mathrm{La}_{1.97} \mathrm{Sr}_{0.03} \mathrm{CuO}_{4}$ in the regime of spin stripes," unpublished (2002).

481. H. V. Kruis, Z. Nussinov, and J. Zaanen, "Emergent $Z_{2}$ gauge symmetry and spin-charge separation in one dimensional physics," cond-mat/0110055 (2001).

482. M. Matsuda, M. Fujita, K. Yamada, R. J. Birgeneau, M. A. Kastner, H. Hiraka, Y. Endoh, S. Wakimoto, and G. Shirane, "Static and dynamic spin correlations in the spin-glass phase of slightly doped $\mathrm{La}_{2-x} \mathrm{Sr}_{x} \mathrm{CuO}_{4}, "$ Phys. Rev. B, 62, 9148-9154 (2000).

483. A. N. Lavrov, Y. Ando, S. Komiya, and I. Tsukada, "Unusual magnetic susceptibility anisotropy in untwinned $\mathrm{La}_{2-x} \mathrm{Sr}_{x} \mathrm{CuO}_{4}$ single crystals in the lightly doped region," Phys. Rev. Lett., 87, 017007-017010 (2001).

484. B. Lake, G. Aeppli, T. E. Mason, A. Schroder, D. F. McMorrow, K. Lefmann, M. Isshiki, M. Nohara, H. Takagi, and S. M. Hayden, "Spin gap and magnetic coherence in a clean high-temperature superconductor," Nature, 400, 43-46 (1999).

485. T. Noda, H. Eisaki, and S. I. Uchida, "Evidence for one-dimensional charge transport in $\mathrm{La}_{2-x-y} \mathrm{Nd}_{y} \mathrm{Sr}_{x} \mathrm{CuO}_{4}, "$ Science, 286, 265-268 (1999).

486. S. Tajima, T. Noda, H. Eisaki, and S. Uchida, "C-axis optical response in the static stripe ordered phase of the cuprates," Phys. Rev. Lett., 86, 500-503 (2001).

487. I. Iguchi, T. Yamaguchi, and A. Sugimoto, "Diamagnetic activity above $\mathrm{T}_{c}$ as a precursor to superconductivity in $\mathrm{La}_{2-x} \mathrm{Sr}_{x} \mathrm{CuO}_{4}, "$ Nature, 412, 420-23 (2001).

488. Y. Wang, Z. A. Xu, T. Kakeshita, S. Uchida, S. Ono, Y. Ando, and N. P. Ong, "Onset of the vortexlike Nernst signal above $T_{c}$ in $\mathrm{La}_{2-x} \mathrm{Sr}_{x} \mathrm{CuO}_{4}$ and $\mathrm{Bi}_{2} \mathrm{Sr}_{2-y} \mathrm{LaCuO}_{6}, "$ Phys. Rev. B, 64, 224519-224528 (2001).

489. Z. A. Xu, N. Ong, Y. Wang, T. Kakeshita, and S. Uchida, "Vortex-like excitations and the onset of superconducting phase fluctuation in underdoped $\mathrm{La}_{2-x} \mathrm{Sr}_{x} \mathrm{CuO}_{4}, "$ Nature, 406, 486-488 (2000).

490. I. Ussishkin, S. L. Sondhi, , and D. A. Huse, "Gaussian superconducting fluctuations, thermal transport, and the Nernst effect," cond-mat/0204484.

491. A. G. Loeser, Z.-X. Shen, D. S. Dessau, D. S. Marshall, C. H. Park, P. Fournier, and A. Kapitulnik, "Excitation Gap in the Normal State of Underdoped $\mathrm{Bi}_{2} \mathrm{Sr}_{2} \mathrm{CaCu}_{2} \mathrm{O}_{8+\delta}, "$ Science, 273, 325-329 (1996).

492. R. S. Decca, H. D. Drew, E. Osquiguil, B. Maiorov, and J. Guimpel, "Anomalous proximity effect in underdoped $\mathrm{YBa}_{2} \mathrm{Cu}_{3} \mathrm{O}_{6+x}$ josephson junctions," Phys. Rev. Lett., 85, 3708-3711 (2000).

493. M. K. Crawford, M. N. Kunchur, W. E. Farneth, and E. M. McCarron, "Anomalous oxygen isotope effect in $\mathrm{La}_{2-x} \mathrm{Sr}_{x} \mathrm{CuO}_{4}$," Phys. Rev. B, 41, 282287 (1990).

494. J. C. Phillips and J. Jung, "Nanodomain structure and function of high temperature superconductors," Phil. Mag. B, 81, 745-756 (2001).

495. S. A. Kivelson and V. J. Emery, "Stripes and Related Phenomena," 91 (Kluwer Academic/Plenum Publishing, New York) (2000).

496. O. Zachar, "Stripes disorder and correlation lengths in doped antiferromagnets," Phys. Rev. B, 62, 13836-13839 (2000). 
497. N. Hasselmann, A. H. Castro Neto, C. Morais Smith, and Y. Dimashko, "Striped phase in the presence of disorder and lattice potentials," Phys. Rev. Lett., 82, 2135 (1999).

498. R. T. Scalettar, G. G. Batrouni, A. P. Kampf, and G. T. Zimanyi, "Simultaneous diagonal and off-diagonal order in the Bose-Hubbard Hamiltonian," Phys. Rev. B, 51, 8467-8480 (1995).

499. S. R. White and I. Affleck, "Density matrix renormalization group analysis of the Nagaoka polaron in the two-dimensional t-J model," Phys. Rev. B, 64, 024411-024416 (2001).

500. D. A. Bonn, K. Zhang, S. Kamal, R. Liang, P. Dosanjh, W. N. Hardy, C. Kallin, and A. J. Berlinsky, "Evidence for rapid suppression of quasiparticle scattering below $\mathrm{T}_{c}$ in $\mathrm{YBa}_{2} \mathrm{CuO}_{7-\delta}$," Phys. Rev. Lett., 72, 1391-1391 (1994). 